\title{
Prevention and treatment of obesity
}

Citation for published version (APA):

van Rinsum, C. E. (2018). Prevention and treatment of obesity: the role of lifestyle coaches and health brokers. [Doctoral Thesis, Maastricht University]. Datawyse / Universitaire Pers Maastricht. https://doi.org/10.26481/dis.20181101cr

Document status and date:

Published: 01/01/2018

DOI:

$10.26481 /$ dis.20181101cr

Document Version:

Publisher's PDF, also known as Version of record

\section{Please check the document version of this publication:}

- A submitted manuscript is the version of the article upon submission and before peer-review. There can be important differences between the submitted version and the official published version of record.

People interested in the research are advised to contact the author for the final version of the publication, or visit the DOI to the publisher's website.

- The final author version and the galley proof are versions of the publication after peer review.

- The final published version features the final layout of the paper including the volume, issue and page numbers.

Link to publication

\footnotetext{
General rights rights.

- You may freely distribute the URL identifying the publication in the public portal. please follow below link for the End User Agreement:

www.umlib.nl/taverne-license

Take down policy

If you believe that this document breaches copyright please contact us at:

repository@maastrichtuniversity.nl

providing details and we will investigate your claim.
}

Copyright and moral rights for the publications made accessible in the public portal are retained by the authors and/or other copyright owners and it is a condition of accessing publications that users recognise and abide by the legal requirements associated with these

- Users may download and print one copy of any publication from the public portal for the purpose of private study or research.

- You may not further distribute the material or use it for any profit-making activity or commercial gain

If the publication is distributed under the terms of Article $25 \mathrm{fa}$ of the Dutch Copyright Act, indicated by the "Taverne" license above, 


\section{Prevention and Treatment of Obesity}

The Role of Lifestyle Coaches and Health Brokers

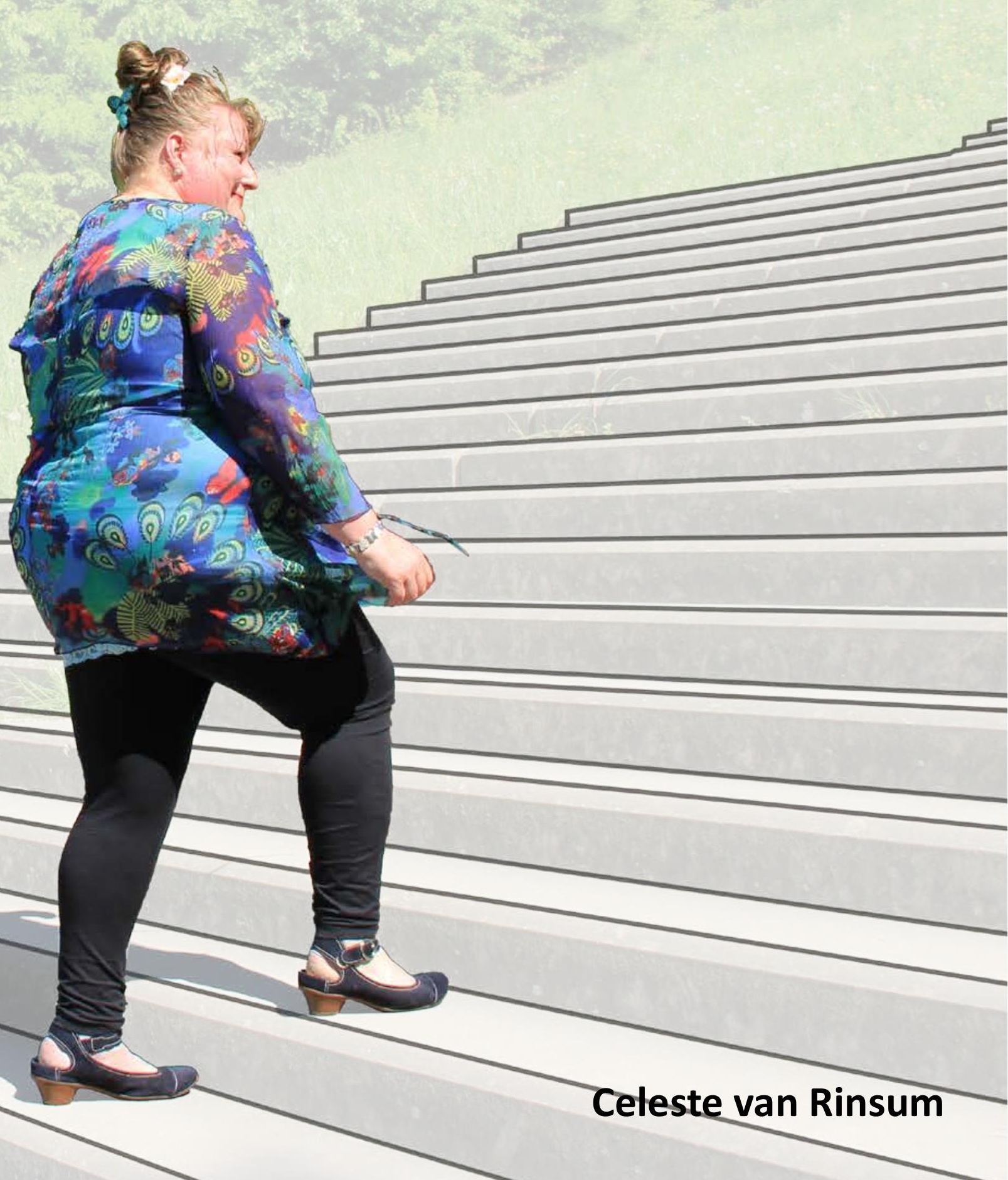


The cover photo illustrates CooL participants setting feasible and achievable action steps for a healthier lifestyle. The other illustrations on the stairs represent different ways of being physically active.

\section{Colophon}

Cover photo: Celeste van Rinsum

Cover and design: Ceciel van Rinsum

Production: Datawyse | Universitaire Pers Maastricht

(C) Celeste van Rinsum, Maastricht, 2018

ISBN: 9789463800037

All rights reserved. Illustrations and brief excerpts from this thesis may be used for scientific and educational purpose provided that the source is acknowledged.

The research presented in this thesis was conducted at the Department of Health Promotion, NUTRIM School of Nutrition and Translational Research in Metabolism, Maastricht University in collaboration with the Department Tranzo, Tilburg School of Social and Behavioral Sciences, Tilburg University. The work of this thesis was supported by health insurance company CZ (project number 20140052). The sub-study 'health brokers' was commissioned by the Northern Limburg Public Health Services. 


\title{
Prevention and Treatment of Obesity The Role of Lifestyle Coaches and Health Brokers
}

\author{
Proefschrift \\ ter verkrijging van de graad van doctor aan de Universiteit Maastricht, \\ op gezag van de Rector Magnificus, Prof dr. Rianne M. Letschert \\ volgens het besluit van het College van Decanen, \\ in het openbaar te verdedigen \\ op donderdag 1 november 2018 om 16.00 uur \\ door \\ Celeste Elvira van Rinsum \\ geboren op 21 december 1989 te Nijmegen
}




\section{Promotoren}

Prof. dr. S.P.J. Kremers

Prof. dr. L.A.M. van de Goor (Tilburg University)

\section{Copromotoren}

Dr. S.M.P.L. Gerards

Dr. G.M. Rutten

\section{Beoordelingscommissie}

Prof. dr. N.K. de Vries (voorzitter)

Dr. R. Crutzen

Dr. P. van Assema

Prof. dr. H. van de Mheen (Tilburg University)

Prof. dr. S.B. Vos (Eindhoven University of Technology) 


\section{CONTENTS}

Chapter 1 General introduction

Chapter 2 The Coaching on Lifestyle (CooL) intervention for obesity, a study protocol for an action-oriented mixed-methods study 21

Chapter 3 Coaching on Lifestyle (CooL) intervention: the lifestyle coach as a linchpin?

Chapter 4 The Coaching on Lifestyle (CooL) intervention for overweight and obesity: a longitudinal study into participants' lifestyle changes

Chapter 5 The implementation of the Coaching on Lifestyle (CooL) intervention: lessons learnt

Chapter 6 Health brokers: how can they help deal with the wickedness of public health problems?

Chapter 7 General discussion

Chapter 8 Appendix

Valorisation

Summary

Samenvatting

References

Dankwoord

Curriculum vitae 


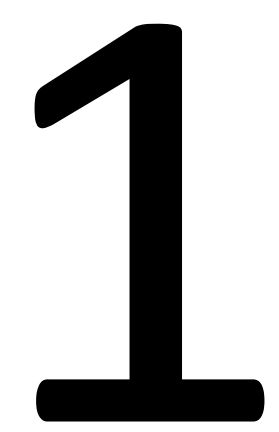

General introduction

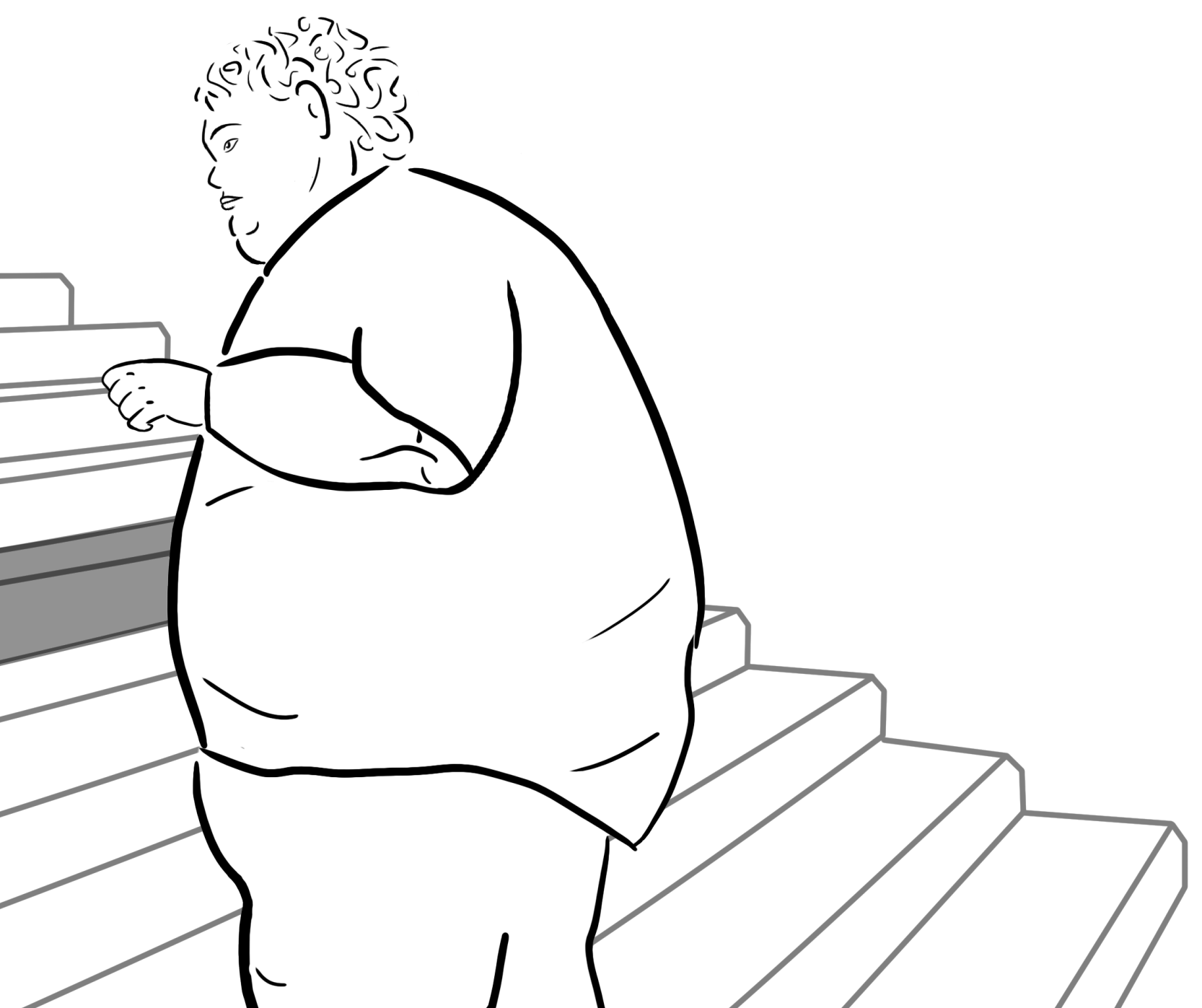





\section{OVERWEIGHT AND OBESITY}

The prevalence of overweight and obesity is increasing worldwide, and has reached nearly $30 \%$ of the global population [1-3]. In the Netherlands, $49.9 \%$ of the adult population are overweight, $14.2 \%$ of whom are obese [4]. The number of children with overweight and obesity in particular has rapidly increased in recent decades [5]. Currently, $13.3 \%$ of the children in the Netherlands are overweight and $2.8 \%$ are obese [4]. A high BMI contributed to $7.1 \%$ of worldwide deaths from any cause, and $39 \%$ of deaths were associated with high BMI-related problems in persons ranging from underweight to overweight [6].

Weight status is classified on the basis of body mass index (BMI) into five categories, according to international guidelines [7]: normal weight $\left(18.5 \leq \mathrm{BMI}<25 \mathrm{~kg} / \mathrm{m}^{2}\right)$, overweight $\left(25 \leq \mathrm{BMI}<30 \mathrm{~kg} / \mathrm{m}^{2}\right)$, obesity $\left(30 \leq \mathrm{BMI}<35 \mathrm{~kg} / \mathrm{m}^{2}\right)$, severe obesity $(35 \leq \mathrm{BMI}$ $<40 \mathrm{~kg} / \mathrm{m}^{2}$ ) and morbid obesity (BMI $\left.\geq 40 \mathrm{~kg} / \mathrm{m}^{2}\right)$. Since overweight is defined as a prestage of obesity, the term overweight also includes obesity in this thesis.

Being overweight is caused by an imbalance in energy balance-related behaviours [8]. Briefly, this imbalance is due to excess energy intake and not enough energy expenditure, involving insufficient levels of physical activity, too much sedentary behaviour [9], too much high-energy dietary intake [10] and sleep deprivation [11, 12]. Sleep deprivation may lead to a decrease in physical activity and an increase in energy intake due to disturbed hormone levels. Overweight and obesity have a variety of consequences, both at personal (e.g. medical problems) and at societal level (e.g. huge global economic impact) $[3,13]$.

Obesity is defined as a chronic disease, which requires prevention, diagnosis and treatment [14]. Due to the complexity of obesity [8], this problem must be tackled by addressing multiple components in order to achieve lifestyle changes [15], involving professionals of different care sectors [16]. This integrated or intersectoral approach is often regarded as the best way to prevent overweight and obesity [17].

\section{OBESITY HEALTH CARE IN THE NETHERLANDS}

\section{Health policy}

Overweight is one of the top priorities in the Dutch health policies, which also recommend an integrated and multidisciplinary approach to decrease its prevalence, and the complex problem is being addressed by a wide range of professionals [14]. In addition, for over a decade now, prevention and health promotion in general have been a key point of the Dutch health policy and have gained a prominent place in the national health care system [18-20]. In 2007, the Minister of Health, Welfare and Sports introduced a health policy that aimed to reduce health care expenses by means of a greater focus on prevention $[16,21]$. Current policy focusses less on the absence of disease and more on what people are still able to do, regardless of their diseases [22, 23]. In this per- 
spective, health is viewed as a way to achieve other goals, such as a better quality of life, rather than as a goal in itself. Health policy focusses on individual choices and a physical environment which makes it easy for individuals to make healthy choices. To encourage a healthier life, the minister supported updates of the Dutch dietary guidelines and the guidelines for physical activity. These two sets of guidelines have been reviewed and simplified in 2015 and 2017, respectively, based on new scientific insights [22, 24, 25].

\section{Obesity care}

Overweight and obesity can be prevented and treated at several levels within the Dutch health care system, which includes public health, primary, secondary and tertiary care. The public health sector offers preventive actions by organisations (e.g. public health services and youth health care), activities by social workers to increase people's participation in society, and informal care by relatives and others [26]. Primary care is typically the first point of contact for health care for most people. People can directly contact the primary care providers, including general practitioners, practice nurses, dieticians and physiotherapists. Access to secondary care, i.e. specialised care (e.g. by a surgeon) requires referral from primary care. Referral from primary or secondary care is needed for tertiary care (e.g. specialised centres and university hospitals) [26].

General practitioners and practice nurses play a central role, mainly for adult patients, in diagnosing and addressing overweight and obesity and referring them to other professionals if necessary. Nevertheless, a substantial proportion of general practitioners are not motivated to refer patients to lifestyle interventions, which is caused by their perceived subjective norm and behavioural control [27]. General practitioners should also provide basic lifestyle guidance [28], but the advice about nutrition and physical activity given by them is typically quite general and is given infrequently [29].

In most general practices, it is the specialised practice nurses who are responsible for the prevention and management of chronic diseases, such as type 2 diabetes. They are trained to provide more detailed guidance on lifestyle changes, by means of communication techniques such as motivational interviewing. However, it has been shown that they could improve the quality of their lifestyle counselling so as to have more impact, which should also be more fully integrated in their training programme [30].

The key public health care providers for children are the Youth Health Care (YHC) nurses and physicians, who have multiple systematic contact moments with every child in the Netherlands. One to three extra consultations are available for the prevention or treatment of overweight. This is called the Bridging Plan, which is a systematic approach in which YHC professionals diagnose children with overweight, and advise and counsel them to prevent obesity [31]. Obese children are counselled by paediatricians in secondary care, mostly in collaboration with a dietician, physiotherapist and psychologist [32]. 


\section{Health insurance}

Only a few types of health care costs concerning the prevention and treatment of obesity are reimbursed by Dutch health care insurers or municipal authorities. For children, the one to three extra consultations in the Bridging Plan are reimbursed in most municipalities [31]. Care by general practitioners is covered by the obligatory health care insurance, as is lifestyle advice for people with overweight or obesity by their general practitioner [33]. Additionally, three contact hours of dietary advice are covered by the health insurance. Next to this, individual health care insurers have various agreements about reimbursement of overweight and obesity prevention and care, which also depends on the insurance package chosen. For example, most insurers reimburse treatment by physiotherapists. Each health care insurance policy involves an excess which has to be paid by the patient before treatments are reimbursed. Municipal authorities are responsible for funding prevention programmes, such as local sports facilities [22].

So far, no comprehensive programme for obesity is reimbursed in any form, because health insurers see this as prevention, which should not be part of the insurance. However, in 2007 the Ministry of Health, Welfare and Sports initiated the development of a programme called BeweegKuur. BeweegKuur is a combined lifestyle intervention (CLI; see the paragraph about CLIs later in this introduction) for people with overweight or obesity, supported by a general practitioner, a lifestyle advisor, a physiotherapist and a dietician [34]. In 2009, the ministry planned to include CLIs in the basic compulsory health insurance coverage [35]. However, a new government abandoned this plan in 2010, due to high costs in the short term and the financial crisis of 2008 [36, 37]. This had a negative influence on the BeweegKuur programme, as this lack of funding by the government was a barrier for most primary care practices to continue the intervention $[16,38]$. Based on the developments of this process, the health insurance company $C Z$ initiated the evaluation of a new programme. The ultimate aim of this pilot was to have an optimal programme design for the covering CLIs in the Dutch health insurance system, in order to reduce the health care costs in the longer term [39].

\section{PREVENTION PYRAMID}

One of the options is to prevent and treat overweight and obesity by means of interventions. The various intervention types for obesity can be categorised into five specific levels, ranging from collective prevention to intensive treatment, as depicted in the socalled prevention pyramid (Figure 1). The prevention pyramid is included in the Dutch integrated health care guideline on obesity [14]. This guideline describes the diagnosis as well as the early detection of high-risk individuals and the treatment for people who are overweight or obese $[14,28]$. The division into intervention types is based on the principles of stepped care, which means that care starts at the least intensive and cheapest treatment, and the intensity can be increased when this first treatment has insufficient effect [14].

Understanding the differences between the levels first requires an explanation of the diagnoses that are associated with the specific types of prevention or care. The levels 
in the prevention pyramid are based on the weight-related health risk, which is a combination of the body mass index (BMI) and potential or diagnosed co-morbidity (Table 1). For instance, a person with a BMI of 31 and with diabetes has a severely increased health risk. Specific guidelines describe the assessment of increased risks for type 2 diabetes (DM2) and cardiovascular diseases (CVD) [40, 41].

The first level of the pyramid for prevention and treatment of obesity is a combination of universal and selective prevention, which are both forms of collective prevention. This level focusses on the population as a whole or on large subgroups of the population [42]. The goal of the universal prevention type is to reduce the incidence of illness or risk factors. Examples of interventions are the 'Healthy School approach' (i.e. a systematic and integrated approach to increase the health and healthy lifestyle of pupils and teachers) [43], changes in the physical environment (e.g. creating bicycle paths), laws and mass media campaigns. Selective prevention targets population groups which are at increased risk for a particular disease [42]. It aims to increase the health of this group and to decrease the risk that the disease will become worse, by means of early detection. Examples of risk groups include people with a low socio-economic status or low health literacy. These risk groups can be targeted with interventions, such as education on the topics of physical activity and healthy nutrition.

The second prevention level is that of indicated or targeted prevention, focussing on individuals with symptoms, but without a disease having been diagnosed [42]. As regards the prevention of obesity, for instance, indicated prevention may be aimed at people who are overweight, but not obese. The goal is to prevent the development of the disease and further health damage by means of a preventive intervention. There is, however, a lack of proven and cost-effective indicated prevention interventions for adults [14]. Instead, health care providers can enhance patients' awareness of their lifestyle and can discuss changes they can already make by themselves. As regards childhood obesity, indicated prevention interventions are offered in Dutch municipalities for parents and their overweight children. An example of such interventions are the extra consultations from YHC professionals about themes such as physical activity and nutrition [31].

Finally, the top of the pyramid involves treatment and maintenance at three levels of risk severity. Treatment and maintenance is a prevention level for people who have already developed a disease, with the aim of reducing the disease burden and the risk of co-morbidity, and improving their self-management $[14,42]$. The preferred treatment type, as described in the health care guideline on obesity, is a CLI. CLIs consist of components relating to physical activity, diet and behaviour [14]. Only if the CLI treatment is unsuccessful can people be given medication. The highest level of risk severity requires a prevention level including a specialised treatment and maintenance approach. Such treatment may consist of cognitive behavioural therapy as a standard part of their treatment besides the CLI. Bariatric surgery is only an option if the CLI has proved unsuccessful after one year, and under strict criteria [14]. 


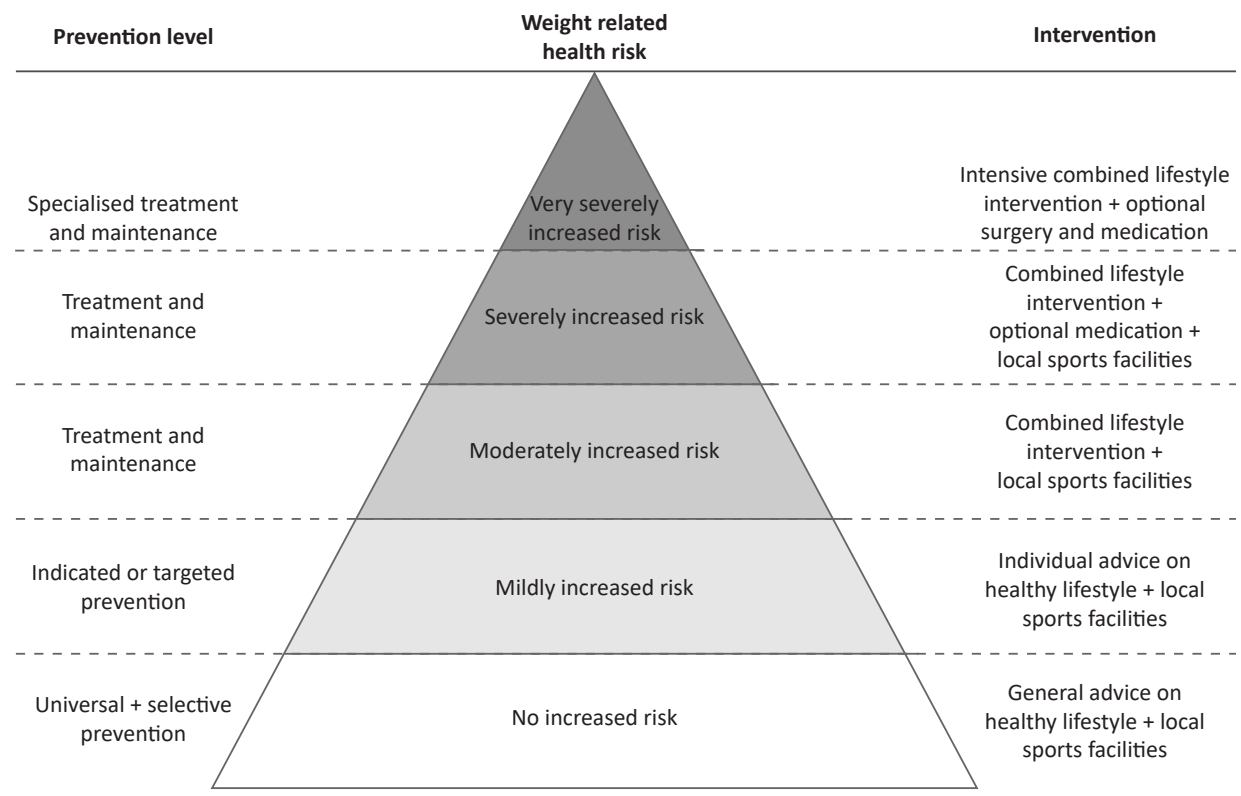

Figure 1: Levels of prevention and treatment for obesity, based on the Dutch integrated health care guideline on obesity [28]

Table 1: Levels of weight-related health risk for adults [28]

\begin{tabular}{llll}
\hline $\mathrm{BMI} \mathrm{kg} / \mathrm{m}^{2}$ & $\begin{array}{l}\text { No increased risk of DM2 } \\
\text { and CVD }\end{array}$ & $\begin{array}{l}\text { Increased risk for DM2 } \\
\text { and CVD }\end{array}$ & Co-morbidity** \\
\hline$\geq 25 \mathrm{BMI}<30$ & Mildly increased & Moderately increased & Moderately increased \\
$\geq 30 \mathrm{BMI}<35$ & Moderately increased & Moderately increased & Severely increased \\
$\geq 35 \mathrm{BMI}<40$ & Severely increased & Severely increased & Very severely increased \\
$\mathrm{BMI} \mathrm{40 \geq}$ & Very severely increased & Very severely increased & Very severely increased \\
\hline
\end{tabular}

Notes: ${ }^{*}>5 \%$ increased mortality risk of CVD and/or increased risk assessed by a type 2 diabetes risk score, which includes waist circumference, family history of type 2 diabetes, presence of hypertension, physical inactivity as well as diagnosis of impaired fasting glucose, ** DM2, CVD, sleep apnoea and/or arthritis.

\section{COMBINED LIFESTYLE INTERVENTIONS}

People who are overweight or obese are supported by CLIs in initiating and maintaining healthier lifestyle behaviours [44]. These interventions include physical activity, dietary and behavioural components [45], and are typically carried out by general practitioners, practice nurses, physiotherapists, psychologists and/or dieticians [46]. Behavioural techniques used in CLIs are strategies to support lifestyle changes in line with behaviour change theories, for example self-monitoring, goal setting and relapse prevention [47]. It is the combination of topics, relative to the individual components, which provides the added value of CLIs. There are many international and Dutch examples of CLIs, both 
for adults [38, 48-51] and for children [52-57].

Randomised clinical trials (RCTs) have shown that CLIs can successfully support adults $[45,58,59]$ and children [60-62] in changing their lifestyle behaviours and body weight. Although evidence from these relatively controlled research settings is promising, the challenge is to make these interventions suitable for 'real-world' settings, in view of the varying and complex contexts of each setting $[63,64]$. It has now become more common to implement CLIs in real-world settings, to understand their effect outside the clinics. For example, the Diabetes Prevention Program has been translated to several real-world settings [65-67]. The conclusions of these real-world effectiveness studies were in line with those of the studies in controlled settings, although effect sizes were generally smaller than those found in highly controlled studies [68]. However, the lifestyle effects of CLIs and other types of weight loss interventions have proved to be rather short-lived; relapses in terms of weight gain in the long term are common [69].

It is not only the effectiveness of CLIs which has been studied, but the implementation process of CLIs in real-world settings has also been investigated [35, 53, 70-73]. These process evaluations have identified implementation barriers in terms of a lack of multidisciplinary collaboration and insufficient skills among primary care professionals, as well as lack of time to optimise coaching $[35,72]$. One of the major barriers for patients has typically been the fact that their health insurance did not fully cover the costs of the interventions [72]. In addition, evaluations have indicated that parents play a key role in determining their children's lifestyle behaviour [74], so both parents and their children are advised to participate in CLIs.

It can be concluded that CLIs can successfully change people's lifestyle, but they have to be further developed in terms of the skills of the professionals, higher frequency of sessions, and programmes offered free of charge for participants, to maintain these changes.

\section{THE COACHING ON LIFESTYLE INTERVENTION}

Insights into implementation issues obtained from previous intervention studies in both adults and children, as described above, have led to the design of the Coaching on Lifestyle (CooL) intervention. The CooL intervention targets people who are obese or at high risk of obesity, that is, ranging from moderately increased weight-related health risk to very severely increased risk. It consists of lifestyle coaching in frequent group and individual sessions, addressing the topics of all major behavioural areas linked to obesity and lifestyle, i.e. physical activity, dietary behaviours, sleep, stress management and behavioural change. The inclusion of the topics of sleep and stress management is relatively new and offers added value compared to most other CLIs. Various evidence-based techniques and approaches have been incorporated in the basic programmes, such as autonomy-supportive coaching, goal setting and ownership. 
During the process of changing the participants' lifestyle, they identify their unhealthy behaviours and are encouraged to replace unhealthy routines by healthier alternatives. The lifestyle coaches stimulate the participants to identify challenging but achievable action steps for each of the behaviours in order to set feasible and sustainable goals that will improve their long term health.

Three different basic programmes focus on three age groups: primary school children (aged 4 to 12 years), adolescents (aged 12 to 18 years) and adults (aged 18 years and older). In the children's and adolescents' programmes, parents participate together with their child. The group sessions are held in locations as close to the participants' residences as possible. Only the individual sessions for the children and adolescents take place at their own homes. The programme as a whole lasts approximately 8 to 10 months.

In addition to these basic programmes, coaches can, if required, assess the needs of each participant and the appropriateness of being included in a relapse prevention intervention (group and individual sessions) or additional individual sessions. These supplementary programmes have been set up to prevent relapse and to maintain the healthy behaviours in the longer term.

During the pilot period described in this thesis, the intervention was reimbursed by health insurance companies and, therefore, free of charge for participants to participate. $\mathrm{CZ}$, one of the major Dutch health insurance companies, initiated the process of developing CooL, and financed a pilot study to evaluate the implementation of this intervention.

In the CooL study, lifestyle changes achieved by participants over time were examined in a one-group pre-post design using mixed methods. The study used an action-oriented approach, meaning that the research team was closely involved in the implementation process, to monitor the implementation of the CooL intervention. Since the main researcher participated in all organisational meetings, this enabled her to observe and simultaneously support the implementation process.

\section{LIFESTYLE COACHES}

In CooL one professional, the lifestyle coach, counsels the participants during the group and individual sessions. This trained coach is a novel professional in Dutch primary care. As described above, evaluations of previous CLIs found that primary care professionals had insufficient skills to counsel participants in changing and maintaining their lifestyle. The goal of CooL was to evaluate the work of coaches with a post-graduate degree and more specific training in lifestyle coaching. The lifestyle coaches in the studies presented in this thesis had completed the post-graduate training programme on lifestyle coaching at the Dutch Academy for Lifestyle and Health (AVLEG). They did not necessarily have a medical degree. CZ chose to collaborate with AVLEG because of the post-graduate level of the training course. 
Lifestyle coaches guide individuals who are obese or at high risk of obesity at the treatment and maintenance level, i.e. the upper part of the prevention pyramid. In addition to counselling the participants, lifestyle coaches act as a single point of contact for the participants and take on a role as linchpins (i.e. having a central and connecting role) in a participant's care provider network. Additionally, they function as links between primary care professionals and public health professionals from whom they receive referrals and to whom they can refer participants.

\section{HEALTH BROKERS}

Another type of professional in the field of prevention is the health broker. In the Netherlands, the role of health brokers was introduced more than a decade ago. Similar to the lifestyle coaches, health brokers act as linchpins in prevention and health care. Unlike the lifestyle coaches, however, they work more at the lower end of the prevention pyramid (the universal and selective prevention level) and at the community level, to connect locally with different parties, such as various municipal government sectors [75]. Note that the study discussed in Chapter 6 of this thesis, which examined the role of health brokers, was unrelated to the CooL pilot study. However, we assumed that the results of the separate health broker study could help to create a more complete picture of the function of brokering in the attempt to link different levels and sectors that operate within the pyramid for prevention and treatment of obesity.

Health brokers aim to create support and establish permanent collaborations, and to encourage knowledge exchange among politicians, private parties, health promotion practitioners, citizens and other stakeholders to improve the health of the community. Additionally, they watch out locally for the most important health problems to tackle and identify opportunities to do so. Health brokers are assumed to operate as 'anchoring points' by connecting community problems to policies and services [76, 77]. An example is that health brokers may facilitate the redesign of a playground, by discussing the health topic and lobbying at the governmental level (e.g. the Department of Spatial Planning) as well as at the operational policy level (e.g. implement health promotion interventions) and with citizens. These brokers have a background in health promotion or in other fields such as social science, and they are employed by the municipal government or the public health services.

\section{BRIDGING THE GAPS}

Obesity prevention will be more successful if multiple levels are addressed. The ideal situation is to provide a preventive environment with rules and regulations that are aligned with educational and counselling interventions [42]. When multiple professionals are linchpins of health promotion, including lifestyle coaches and health brokers, all levels of the prevention pyramid can potentially be linked in such a way that synergy (i.e. an effect of collaboration which is greater than the sum of the parties' individual effects) is induced. 
Currently, there is a gap in the Dutch health care system between the upper and lower parts of the pyramid. While municipal authorities are responsible for providing local sports facilities that can assist in obesity prevention, it is the primary care professionals who advise their patients regarding their engagement in healthier lifestyles, and patients are rarely referred to professionals in the public sector. Additionally, health care providers have been urged to collaborate more fully with each other [14]. Health brokers and lifestyle coaches can play an important role in bridging this gap by taking up the roles of connectors and mediators.

\section{OUTLINE OF THIS THESIS}

The general research question of this thesis is: Can lifestyle coaches and health brokers help to bridge the gap between the prevention and health care domains regarding overweight and obesity? More specifically, this thesis focuses on facilitating and impeding factors concerning the implementation of the CLI called CooL, and on behaviours and determinants of the health brokers' role at the community level in the Netherlands.

Chapter 2 presents the protocol of the evaluation study of the CooL intervention, including a description of the intervention and the qualitative and quantitative measurements used in the study (see Figure 2 for an overview of this thesis). It describes that the aim of the CooL study is to monitor the implementation of the CooL intervention and to investigate how lifestyle coaches contribute to a healthier lifestyle of the participants.

Chapter 3 summarises the results of the CooL study, focussing mainly on the process evaluation of implementing the CooL programme, and briefly describes the lifestyle changes achieved by the CooL participants over time. In essence, this is a summary of the extensive research report on the project, which is also available online (in Dutch) $[78]^{*}$.

A more detailed description of the participants' lifestyle changes over time is presented in Chapter 4. It discusses the results of the CooL intervention as identified in a longitudinal one-group pre-post study using validated questionnaires and objective data. The study investigated the motivational, behavioural, quality of life and weight changes achieved by the CooL participants, during the intervention period as well as in the longer term.

Chapter 5 describes the detailed results of the evaluation of the CooL implementation process and the facilitating and impeding factors in this process, using a mixed methods research design and an action-oriented approach.

Chapter 6 reports on the primary behaviours and determinants of the health brokers' role. This study explored the role of health brokers regarding emerging wicked health

* https://www.maastrichtuniversity.nl/nl/nieuws/leefstijlcoach-motiveert-mensen-met-overgewicht-om-gezonder-te-leven 
problems, such as obesity. Based on interviews, the model called 'Health Broker Wheel' was developed, a framework depicting the health brokers' role.

A general discussion of the PhD project and its conclusions is presented in Chapter 7, in which the findings of the individual chapters are integrated and discussed in the light of a broader view on obesity prevention, while methodological considerations regarding the research process are outlined and an overall conclusion is presented.

Chapter 2: Study protocol of the CooL intervention

Chapter 3: Summary of the results from the CooL intervention

Chapter 4: Longitudinal lifestyle changes of CooL participants

Chapter 5: Process evaluation of the CooL implementation

Chapter 6: Behaviours and determinants of the health brokers' role

Figure 2: General outline of this thesis 


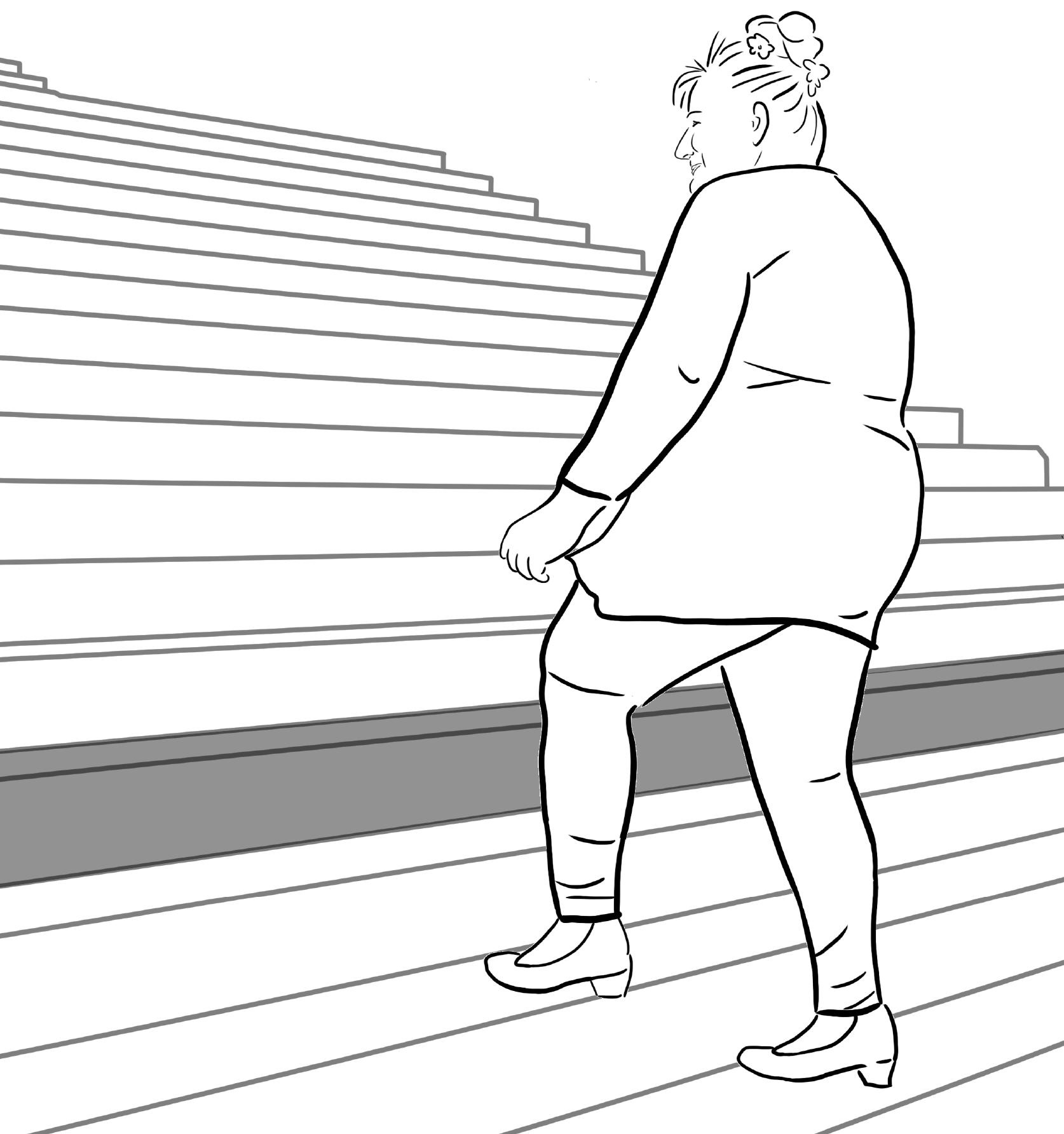




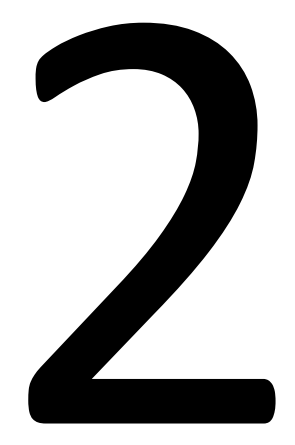

\section{The Coaching on Lifestyle (CooL) intervention for obesity, a study protocol for an action- oriented mixed-methods study}

Celeste E van Rinsum, Sanne MPL Gerards, Geert M Rutten, len AM van de Goor, Stef PJ Kremers

BMC Public Health, 2018; 18: 117 


\begin{abstract}
Background: Combined lifestyle interventions (CLIs) have proved to be effective in changing and maintaining behavioural lifestyle changes and reducing overweight and obesity, in clinical and real-world settings. In this CLI, lifestyle coaches are expected to promote lifestyle changes of participants regarding physical activity and diet. In the Coaching on Lifestyle (CooL) intervention, which takes a period of 8 to 10 months, lifestyle coaches counsel adults and children aged 4 years and older (and their parents) who are obese or are overweight with an increased risk of developing cardiovascular diseases or type II diabetes. In group and individual sessions, themes such as physical activity, dietary behaviours, sleep and stress are addressed. The aim of the present study is to monitor the implementation process of the CooL intervention and to examine how the lifestyle coaches contribute to a healthier lifestyle of the participants.
\end{abstract}

Methods: This action-oriented study involves monitoring the implementation process of the CooL intervention and examining the lifestyle changes achieved by participants over time, in a one-group pre-post design using mixed methods. Methods include semi-structured interviews, observations, document analysis, biomedical parameters and questionnaires.

Discussion: The added value of the CooL study lies in its action-oriented approach and the use of mixed methods, including both qualitative and quantitative research methods. The long-term coaching used in the CooL intervention is expected to have beneficial effects on sustained lifestyle changes.

Trial registration: NTR6208; date registered: 13-01-2017. 


\section{BACKGROUND}

Half of the Dutch adult population are currently overweight or obese, as well as $12 \%$ of the children [4]. These lifestyle-related health problems have a variety of consequences, both at personal (e.g. medical problems) and at societal level (e.g. huge global economic impact) $[3,13]$. Overweight is caused by an imbalance in energy balancerelated behaviours, which are complex behaviours with many underlying factors [8, 79]. Combined lifestyle interventions (CLIs) seem to be suitable interventions to support persons in initiating and maintaining changes in diet and physical activity [44].

Previous randomised clinical trials (RCTs) have shown that CLIs can successfully support children $[60,61]$ and adults $[45,58]$ in changing their lifestyle behaviours and body weight. In the Netherlands, for example, the SLIM intervention was found to be effective in reducing diabetes incidence and body weight, and in improving dietary habits among adults [80]. Although evidence from relatively controlled research settings is promising, the challenge is to make these interventions suitable for 'real-world' settings [63].

Recent studies have investigated the implementation of CLIs, such as the BeweegKuur [38], SLIMMER [50] and MetSLIM [51] programmes, in real-world settings in the Dutch context for patients with type II diabetes or obesity. Lifestyle Triple P [54] and COACH [57] are Dutch examples of interventions in real-world settings for children with obesity. The conclusions of these effectiveness studies were in line with those of the controlled studies, although effect sizes were generally smaller than those found in highly controlled studies. These programmes have been shown to have positive effects on physical activity and dietary behaviours, often accompanied by improved quality of life and a decrease in body mass index (BMI), waist circumference and other metabolic risk factors $[38,50,51,54,57]$.

However, process evaluations of real-world CLIs have indicated implementation barriers in terms of a lack of multidisciplinary collaboration and insufficient skills among primary care professionals, as well as lack of time to optimise coaching [35, 72]. One of the major barriers for patients was the fact that their health insurance did not fully cover the costs of the intervention [72].

The identification of implementation issues, such as those reported above, has led to the design of the Coaching on Lifestyle $(\mathrm{CooL})$ intervention. The lifestyle coaches in this intervention are professionals with special postgraduate training and play a key role in the intervention by coaching adults and children, who are obese or are at high risk of obesity, to help them achieve a sustained healthier lifestyle. These lifestyle coaches are thus expected to occupy a new position in the Dutch health care system [81-83], are currently in an experimental condition financed under the basic health insurance system and will function as linking pins between primary care professionals and public health professionals. In group and individual sessions, the lifestyle coaches address themes such as physical activity, dietary behaviours, sleep and stress. 
The aim of the present study is to monitor the implementation of the CooL intervention and to investigate how lifestyle coaches contribute to a healthier lifestyle of the participants. This study uses an action-oriented approach, implying that results of observations are also used as input to improve the content or implementation process of the intervention. The assumption is that when opportunities and barriers are identified and adjustments are made, valid recommendations can be made for optimising the role of the lifestyle coaches in the prevention chain of chronic lifestyle-related health problems, such as obesity and overweight.

\section{METHODS}

\section{Design}

In this action-oriented study we monitor the implementation process of the CooL intervention and the lifestyle changes achieved by participants over time, in a onegroup pre-post design, using mixed methods. The process is studied by means of group and individual interviews, observations and document analysis (qualitative). The changes over time among participants are examined by means of questionnaires and biomedical parameters (quantitative). This study is expected to provide an indication of the effectiveness of this intervention in terms of the degree to which patients succeed in maintaining their changed behaviours. This study protocol has been approved by the Medical Ethics Committee of the University Hospital Maastricht and Maastricht University (reference number METC 14-5-021).

The theoretical framework of the study is shown in Figure 1. The upper three boxes show the methods and measurements of this study, while the boxes within the dotted lines indicate the behavioural system of the participants, subdivided into adults and children. The energy balance-related behaviours are a combination of energy intake (i.e. dietary behaviours) and energy output (i.e. physical activity). In addition, sleeping behaviour is assumed to influence energy balance. Intervention elements are assumed to impact lifestyle changes of the participants through their influence on motivational regulation and behaviour-specific cognitions. The figure also depicts the context, which is assumed to influence the implementation process of the intervention and thus have a potential moderating influence on the changes achieved by the participants [23]. For example, when referrers are enthusiastic about the intervention, the impact of the intervention is likely to be higher.

\section{Study setting}

The Cool study takes place in different regions within two provinces of the southern part of the Netherlands, i.e. in the province of Noord-Brabant and the southeastern part of the province of Limburg. The CooL intervention and the data collection period for this study started in April 2014, and will continue over a period of three years. 

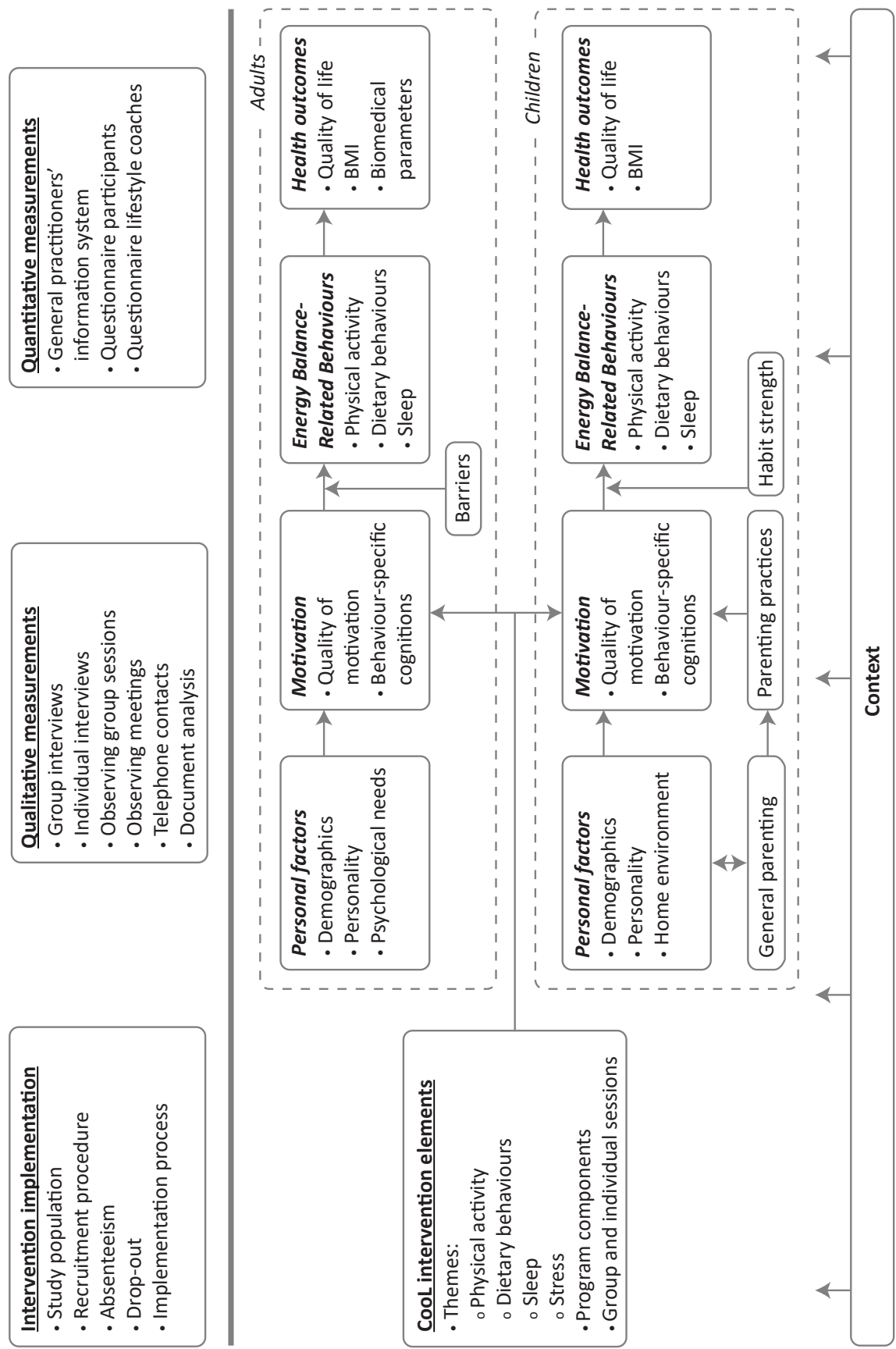

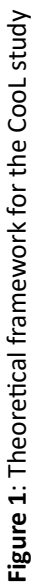




\section{Study population}

\section{Participants}

In this intervention, the lifestyle coaches counsel individuals aged 4 years and older who are obese or are at high risk of obesity (see Appendix 1). These are persons who are either obese $(\mathrm{BMI} \geq 30)$ or are overweight $(\mathrm{BMI} \geq 25)$ and are at increased risk of cardiovascular diseases or type II diabetes, according to the Dutch guidelines on obesity $[14,40,41]$. For children and adolescents (under 18 years), specific BMI cut-off points for the different ages are used [14]. Also, the participants must have a health insurance policy with the Dutch health insurance companies CZ or VGZ or one of their subsidiaries. Exclusion criteria are a lack of motivation to participate in the intervention and being unable to fit in a group (e.g. because of behavioural problems).

\section{Lifestyle coaches}

In all 13 lifestyle coaches participate in the study. All have completed a post-graduate training course at the Dutch Academy for Lifestyle and Health (AVLEG). They are members of the Professional Association of Lifestyle Coaches in the Netherlands (BLCN). A co-founder of the AVLEG has selected the lifestyle coaches, who participate in the project, based on their skills and their willingness to participate.

\section{Recruitment of participants}

There are two pathways of recruitment: participants can either sign up for the intervention themselves or they are referred to the intervention by a health care professional. Practice nurses and general practitioners are instructed by 'health care groups', to which they are affiliated, to refer adult patients. Health care groups are organisations coordinating chronic care for a cluster of health care professionals. In addition, internal medicine specialists from hospitals are instructed by the project leader to refer potential adult participants. The recruitment of children mostly takes place via referral by Youth Health Care agencies (YHC). In addition, general practitioners, neighbourhood sports coaches, schools (such as those taking part in the 'Healthy Elementary School of the Future' programme [84]), pedagogical workers and paediatricians have also been informed about the programme for children and asked to recruit potential participants. Participants that sign up by themselves, are instructed to get a referral from their health care professional.

\section{Sample size calculation}

In a period of three years the goal is to include as much as participants as possible in order to learn optimally from the implementation process, with a maximum of 350 participants per target group (adults / children) due to financial resources. The sample size calculation is based on physical activity, since this is a primary behavioural goal in the intervention, and we used data from previous studies in similar target populations as a basis $[85,86]$. For a difference of 140 min per week in physical activity (i.e. light activities (walking) and moderate-intensity to vigorous-intensity activities) with $80 \%$ power, $5 \%$ significance (two-sided), a standard deviation of 90 min per day and a dropout of 
$30 \%$, a sample size of 235 adult participants is required. Potential clustering of effects (nesting of effects within the lifestyle coaches) has been corrected for by estimating the intracluster correlation $(\rho)$ for physical activity at 0.006 (corresponding to a design effect or inflation factor of 1.23). In the effectiveness study of Lifestyle Triple P [16], it was concluded that within children a difference of 60 min per week in physical activity can be achieved with an intervention aimed at parents. Assuming a standard deviation of 30 min per day, $30 \%$ dropout and $\rho$ of 0.006 , a sample size of 135 participants is required to identify this difference with $80 \%$ power and $5 \%$ significance.

\section{Study procedure}

The participants are informed in advance about the study using written information and personal information by the lifestyle coaches, and they are asked to give permission for the anonymous use of their biomedical data and the data gathered in the study, by means of an informed consent form. The participants receive four questionnaires to fill in: at baseline (TO); approximately 8 to 10 months after baseline, at the programme's end (T1); 18 months after baseline (T2); and 24 months after baseline (T3). The baseline questionnaire is handed out by the health care professionals or the lifestyle coaches. The T1 questionnaire is handed out by the lifestyle coaches, and the T2 and T3 questionnaires are sent to the participants by the research team. This procedure and the referral guidelines have also been laid down in a protocol for the lifestyle coaches and referrers.

\section{CooL intervention}

\section{Programmes}

Three programmes have been developed for the different age groups, based on examination of existing evidencebased programmes, theory-based literature and practice (Table 1):

1. Adults programme (18 years and older)

2. Adolescents programme, for adolescents (12 to 17 years) and their parents

3. Children's programme, for primary school children (4 to 11 years old) and their parents

In the basic programme for adults, participants are coached for 8 to 10 months (see Table 2 for an overview of the sessions and the themes). The groups consist of 4 to 12 participants. The adults attend 1 intake session and 1 outtake session (60 minutes each), 8 group sessions ( 90 minutes each) and 2 individual sessions ( 45 minutes each). During the intake session, the participant's problem is mapped as well as their motivation to change their lifestyle behaviours. The children's programme consists of 1 intake session and 1 outtake session at home with the family (60 minutes each), 8 group sessions with parents and a maximum of 8 individual sessions at home (in total 4 hour). The focus is on providing the parents with tips for healthy parenting styles and for changing their child's lifestyle to a healthier one. The programme for adolescents is a combination of those for the children and the adults, with a greater emphasis on peer influences. The intervention for the adolescents was added at a later stage of the implementation process, to close the gap between children and adults. As a result, our study will only 
Table 1: Number of sessions per target group and per programme

\begin{tabular}{|c|c|c|c|}
\hline Components & Children & Adolescents & Adults \\
\hline \multicolumn{4}{|l|}{ Basic programme } \\
\hline Individual sessions & $10 x$ at home & $10 x$ at home & $4 x$ \\
\hline \multirow[t]{3}{*}{ Group sessions } & $8 x$ for the parents & $5 x$ for adolescents & $8 x$ \\
\hline & & $1 \mathrm{x}$ for parents & \\
\hline & & $\begin{array}{l}2 x \text { for adolescents and } \\
\text { parents }\end{array}$ & \\
\hline Additional programme & - & - & 10x individual sessions \\
\hline $\begin{array}{l}\text { Relapse prevention } \\
\text { programme }\end{array}$ & $\begin{array}{l}\text { Same number of ses- } \\
\text { sions as basic interven- } \\
\text { tion }\end{array}$ & $\begin{array}{l}\text { Same number of ses- } \\
\text { sions as basic interven- } \\
\text { tion }\end{array}$ & $\begin{array}{l}\text { Same number of ses- } \\
\text { sions as basic interven- } \\
\text { tion }\end{array}$ \\
\hline
\end{tabular}

Table 2: Themes group sessions

\begin{tabular}{|c|c|c|c|}
\hline Group sessions & Children & Adolescents & Adults \\
\hline 1 & $\begin{array}{l}\text { Awareness and behaviour } \\
\text { change }\end{array}$ & $\begin{array}{l}\text { Awareness and behaviour } \\
\text { change }\end{array}$ & $\begin{array}{l}\text { Awareness and behaviour } \\
\text { change }\end{array}$ \\
\hline 2 & Physical activity & $\begin{array}{l}\text { Acting as a role model (only } \\
\text { for parents) }\end{array}$ & Physical activity \\
\hline 3 & Nutrition & Physical activity & Structured eating patterns \\
\hline 4 & $\begin{array}{l}\text { Setting boundaries and } \\
\text { rewarding }\end{array}$ & $\begin{array}{l}\text { Nutrition (including } \\
\text { parents) }\end{array}$ & Sleep, relaxing, stresses \\
\hline 5 & Acting as a role model & Snacking & Time-management \\
\hline 6 & Sleep, relaxing, stresses & Sleep and relaxing & Pitfalls \\
\hline 7 & Pitfalls and planning & Stresses and pitfalls & Relapse prevention \\
\hline 8 & $\begin{array}{l}\text { Self-regulation for the } \\
\text { family }\end{array}$ & $\begin{array}{l}\text { Self-regulation for the fami- } \\
\text { ly (including parents) }\end{array}$ & Self-regulation \\
\hline
\end{tabular}

address the children and adults in terms of the implementation process.

Regarding physical activity, the lifestyle coaches ensure contacts with the neighbourhood sports coaches or suppliers of local sports facilities. In order to improve their physical activity levels, participants are invited during the sessions to think about ways of incorporating lowintensity physical activity (and perhaps also higher intensity physical activity) in their daily lives. In addition, the lifestyle coaches can refer participants to other health care professionals, such as a dietician, general practitioner or physiotherapist, when other problems emerge.

If required, during the outtake session the lifestyle coaches assess the needs of each participant and the appropriateness to be included in the relapse prevention programme or the additional programme. The relapse prevention programme for adults comprises the same number of sessions as the basic programme. However, these sessions are spread over a period of 2 years and the content of the topics is more detailed, 
including more attention for the participants' pitfalls and experiences. The additional programme consists of a maximum of 10 individual sessions, in which the personal factors are discussed in depth. Children and adolescents are only eligible for the additional programme.

\section{Coordination of the intervention}

The project leader, an external expert, functions as a project coordinator to start up and to implement the intervention in the different regions and as the chairman of the monthly regional project group meetings. These project groups consist of the lifestyle coaches, a coordinator from the local health care group or from the public health services (PHS), suppliers of local sports facilities and a care purchasing agent of the health insurance company. In addition to these project groups, there is a project steering group which is responsible at a higher level for the overall implementation and for long-term decisions, and meets twice a year. This project steering group includes representatives of a health care group or PHS, a municipal civil servant, a co-founder of AVLEG and the programme manager health care innovation of the health insurance company. Peer feedback meetings are organised twice a year with the lifestyle coaches and their supervisor, during which they address problems that occur in the implementation of the intervention.

\section{Qualitative measurements}

The experiences of participants, lifestyle coaches, project team members and referrers are used to continually improve the intervention and to understand the effects of the intervention on the participants. Their opinions are collected by means of individual interviews, group interviews, observations and document analysis.

\section{Group interviews}

In addition to the information gained with the questionnaires, approximately one group interview is organised with the participants (i.e. the adults and parents) counselled by each lifestyle coach, immediately after the final group session, to gather more detailed information about the intervention and its barriers. These recorded interviews use a semi-structured interview protocol.

\section{Individual interviews}

Interviews are conducted with the different target groups: participants, lifestyle coaches, referrers, project group members and project steering group members. In addition to the group interviews with participants, more in-depth interviews are conducted with participants $(n=5)$ from one intervention group. Interviews are also conducted with the lifestyle coaches $(n=12)$ to gather information about their functioning, their role as pivots in the intervention and the network, and their opinion of the intervention. Furthermore, various referrers $(n=52)$ in each region are interviewed to explore the process of recruiting participants, the logistics of the referral process, barriers in the referral process and points for improvement. Finally, the project group members and project steering group members $(n=12)$ are interviewed to study the implementation process and to assess their opinions about the project group and project steering group 
meetings and the intervention. These interviews are audio-recorded.

\section{Observing group sessions}

One to two participant group sessions (with the theme sleeping and relaxation (for adults session number four and for children session number six), and the last group session) of each lifestyle coach are observed, to study the implementation of the intervention, the process of change within the groups and the development of the intervention over time. In addition, one adult group is observed during the entire programme in order to examine the intervention as a whole.

\section{Observing meetings}

The main researcher or a co-researcher participates in all the project group, steering group and peer feedback meetings to monitor the implementation process and the facilitating and impeding factors for this process. Notes are made of the observations.

\section{Telephone contacts}

Weekly telephone contacts between the project leader and the main researcher are held to keep track of the implementation process and to discuss the current situation. Furthermore, the project team members can always contact the main researcher by telephone or email. Notes of these contacts are again made by the main researcher.

\section{Document analysis}

All minutes from all project meetings, personal notes and e-mails are analysed to monitor the implementation process. Next to this, the lifestyle coaches keep track of absenteeism and drop-out in each intervention group. These documents also record the health care pathway, that is, participant recruitment and throughput towards suppliers of local sports facilities and other health professionals. Lastly, analysis of the intervention protocol of the lifestyle coaches and the participants' workbooks provides detailed insights into the content of the intervention.

\section{Quantitative measurements}

\section{Biomedical parameters}

Biomedical parameters of adults, i.e. objectively measured BMI, HbA1C, blood pressure and fasting glucose, will be retrieved from the general practitioners' information system, provided by the health care group. The general practitioners assess these outcomes for patients at high risk of obesity, while the practice nurses monitor the patients with a chronic disease such as type II diabetes. The research team measures the participants' BMI after the intervention. In addition, medication data and the number of consultations participants had with other health professionals are gathered by the health insurer. Children's baseline BMI is measured by a YHC professional; BMI after the intervention (T1) is measured by the lifestyle coach, and BMI at T2 by the research team.

\section{Questionnaire for participants}

The participants receive four different questionnaires (i.e. T0, T1, T2 and T3), adapted to the three different age groups: the children (10 years and older), their parents and the 
adult participants (18 years and older) receive a different version of the questionnaire. See Figure 1 for the theoretical framework, which includes the behavioural system of the participants. We used validated questionnaires where possible.

\section{Adults' questionnaire}

\section{Demographic characteristics}

The adults are asked at baseline about their personal characteristics, such as gender, date of questionnaire completion, date of birth (to calculate their age), body height and weight, country of birth, highest completed education (as an indicator of socio-economic status), living situation and occupational status. The educational level is subdivided into three categories: low (i.e. no education or only primary education), intermediate (e.g. secondary education), and high (tertiary education). The living situation is categorised into living together with someone (married or cohabiting) and living alone (divorced, unmarried or widowed). The occupational status is divided into being in work (paid work, voluntary work or self-employed) and not working (homemaker, unemployed/job seeker, retired/in early retirement, disabled/incapacitated or in education/ studying). In the follow-up questionnaires participants only fill in their current weight and the date of questionnaire completion.

\section{Personality}

A Dutch translation of the Big Five Inventory $(\mathrm{BFI})[87,88]$ is used to measure five different personality dimensions ( 44 items): extraversion ( 8 items), agreeableness ( 9 items), conscientiousness ( 9 items), neuroticism ( 8 items) and openness ( 10 items). An example question on extraversion is: "I see myself as someone who is talkative." These questions are answered on 5-point Likert scales ranging from totally disagree (1) to totally agree (5).

\section{Psychological needs}

In line with Self-Determination Theory [89] we use the Psychological Need Satisfaction in Exercise scale (PNSE) [90] to measure the perceived psychological needs regarding exercise. The 18 items (with a 5-point Likert scale from totally disagree (1) to totally agree (5)) are divided into three subscales (6 items per subscale): perceived competence, perceived autonomy and perceived relatedness. An example of perceived autonomy is "I feel free to exercise in my own way".

\section{Quality of motivation}

The quality of motivation for physical activity among the adults is measured with the Behavioural Regulation in Exercise Questionnaire (BREQ-3) [91] comprising 23 questions (with a 5-point Likert scale from totally disagree (1) to totally agree (5)). The BREQ-3 consists of six subscales: amotivation ( 4 items), external regulation ( 4 items), introjected regulation ( 3 items), identified regulation ( 4 items), integrated regulation ( 4 items) and intrinsic regulation ( 4 items). An example question of introjected regulation is: "I feel guilty when I don't exercise." 
The Regulation of Eating Behaviour Scale (REBS) [92] is used to measure motivation regarding diet and consists of 24 items, such as "Eating healthy is an integral part of my life". The REBS has the same six subscales as the BREQ-3 (4 items per subscale): amotivation, external regulation, introjected regulation, identified regulation, integrated regulation and intrinsic motivation.

\section{Behaviour-specific cognitions}

At baseline, the participants are asked whether they experience social support for being physically active ( 13 items) and eating a healthy diet (10 items). For example: "In the last 3 months, family members/other persons, who are important to me, have participated together with me in physical activities." Furthermore, a person's self-efficacy is assessed by means of 4 items for both physical activity as well as diet. An example question is: "I think I will be able to be more physically active when I am tired." Lastly, the intention to be physically active or to eat a healthy diet is assessed with 4 items per behaviour. These questions are answered on 5-point Likert scales ranging from never (1) to very often (5) or from totally disagree (1) to totally agree (5).

The adult participants are also asked at baseline about their knowledge of healthy lifestyle norms (4 items). Finally, at baseline there are questions about their previous attempts to lose weight and their reasons to participate in this intervention.

\section{Barriers}

The participants are asked about barriers regarding physical activity (11 items) and diet (10 items), with answering options ranging from totally disagree (1) to totally agree (5), such as "Performing physical activity is hard for me, because I feel ashamed when I'm exercising."

\section{Physical activity level}

The International Physical Activity Questionnaire (IPAQ) [93] is used to measure the physical activity level of the adult participants. They are asked how many days a week and how many minutes a day they walked and engaged in moderate-intensity and vigorous-intensity activities during the past week. The numbers of days for these three activity levels is multiplied by the number of minutes. The number of minutes per week is summed to obtain a total activity score. The sedentary behaviour is assessed with 5 items in different domains (while traveling, at work, watching television, using a computer at home and at leisure), presented in minutes per day [94].

\section{Dietary behaviours}

The shortened Fat List [95] is used to measure dietary behaviours of the adults. They are asked about the number of days (on a scale from never to 7 days a week) they have breakfast, eat warm vegetables, salads or raw vegetables, fruits, consume fruit juices and sugarsweetened beverages (including fruit beverages). The amount of vegetables is calculated by multiplying the number of days by the number of serving spoons a day (on a scale from 1 to 6 or more spoons). The same is done for the number of fruits (days multiplied by pieces [on a scale from 1 to 7 or more pieces]). Participants can fill in the number of slices of bread they eat each day and the type of bread (brown, whole wheat 
or white). They are also asked to report on how many days a week they eat different types of take-away food. Lastly, they are asked how many times a week they eat the following snacks: general snacks, peanuts or nuts, potato chips or cheese, pastries, candy, chocolates and cookies (on a scale of never to 7 times a week). These 7 questions are summed to obtain a total snacking score.

\section{Quality of life}

The adults' quality of life (EQ-5D-3 L) [96] is measured with 5 questions. Each item measures a different health state and uses different answering options: mobility (from "no problem walking" to "confined to bed"), self-care ("no problems" to "unable to wash myself"), usual activities ("no problems" to "unable to perform"), pain/discomfort (none to extreme pain), and anxiety/depression (none to extremely anxious). Each question has three answering options, with lower scores meaning fewer problems.

\section{BMI}

The self-reported BMI is calculated from the reported height and weight. The weight status is classified into five categories according to international guidelines [7]: normal weight $\left(18.5 \leq \mathrm{BMI} \leq 24.99 \mathrm{~kg} / \mathrm{m}^{2}\right)$, overweight $\left(\mathrm{BMI} \geq 25 \mathrm{~kg} / \mathrm{m}^{2}\right)$, obesity (BMI $\geq 30 \mathrm{~kg} /$ $\left.\mathrm{m}^{2}\right)$, severe obesity $\left(B M I \geq 35 \mathrm{~kg} / \mathrm{m}^{2}\right)$ and morbid obesity $\left(B M I \geq 40 \mathrm{~kg} / \mathrm{m}^{2}\right)$.

\section{Process evaluation questions}

The participants can report their experiences with the intervention as a whole in 13 questions, with a 5-point Likert scale ranging from totally disagree (1) to totally agree (5). One of the items is: "I am satisfied with the quality of the CooL intervention." The assessment of the group sessions consists of 11 questions, with answering options ranging from totally satisfied (1) to totally dissatisfied (5). One of the questions is: "How satisfied are you with the content of the group sessions?" For the individual sessions, participants can express their appreciation in 6 questions (with options from totally satisfied (1) to totally dissatisfied (5)). One of them is: "How satisfied are you with the links between the individual sessions with the group sessions?"

Participants are also asked to answer 23 questions (with options from totally disagree (1) to totally agree (5)) about the knowledge and coaching skills of their lifestyle coach. An example of the skills question is: "My lifestyle coach helped me to draft a plan to achieve my goals."

Finally, participants can indicate their perceived results. In 12 questions they are asked to report whether they have achieved their goals. One of these questions is: "I am now living a healthier lifestyle." Additionally, the participants are asked 10 questions to evaluate if the goals they achieved match the intervention themes, for instance: "As a result of participating in the CooL intervention, I have made many small changes."

\section{Parents' questionnaire}

\section{Demographic characteristics}

The children's primary care givers are asked to report the same personal characteristics 
as the adult participants, for themselves, their partner (if any) and the child participating in the CooL intervention, viz. gender, date of filling in, date of birth, height, weight, country of birth, highest completed education, living situation and occupational status. Additionally, they are asked to describe their family composition and situation, the school year that the child is in and whether they use any form of child care.

\section{Personality of the child}

We use the questions on impulsivity from the Temperament in Middle Childhood Questionnaire (TMCQ) [97], which consists of 13 questions. The question "My child makes up its mind suddenly" can be answered on a 5-point Likert scale (with options from almost never applicable (1) to almost always applicable (5)) or choose the answer "never seen my child in this situation."

\section{General parenting}

A shortened version, with 45 items, of the Comprehensive General Parenting Questionnaire (CGPQ) [98] is used to assess general parenting styles, with answering options with a 5-point Likert scale ranging from totally disagree (1) to totally agree (5).

The parental perceptions of their children's behavioural problems regarding overweight and obesity, and the parents' self-efficacy in dealing with these behaviours, are measured with the Lifestyle Behaviour Checklist $(\operatorname{LBC})[99,100]$. Firstly, the parents are asked to report to what degree the child's behaviour is a problem to them ( 25 items). For example, the statement "My child yells about food" is answered on a 7-point Likert scale from not at all (1) to very much (7). Secondly, for the same statement they can grade their own confidence to deal with the problem on a 10-point Likert scale from certain I cannot do it (1) to certain I can do it (10).

\section{Parenting practices}

To assess the parenting practices, 49 items from several validated questionnaires have been combined. Dutch translations of these questions have already been used in other studies. The 4 items on intake monitoring are based on the Child Feeding Questionnaire (CFQ) [101] and the 2 questions on monitoring activity are derived from Gubbels et al. [102]. Stimulation to be active and to eat a healthy diet is assessed by 5 items [102]. The constructs modelling healthy eating ( 4 items), food environment ( 4 items, defined as healthy food being available at home) and child control (5 items) are subscales of the Comprehensive Feeding Practices Questionnaire (CFPQ) [103]. Parental role modelling ( 8 items) and parental policies ( 5 items) for physical activity are derived from the Home Environment Survey (HES) [104]. Emotional (5 items) and instrumental feeding (4 items) are assessed using subscales of the Parental Feeding Style Questionnaire (PFSQ) [105]. Lastly, 3 items from the Covert Control scale [106] are used.

These questions use 5-point Likert scales ranging from totally disagree (1) to totally agree (5) or from never (1) to often (5). An example of emotional feeding is: "I give my child something to eat if $s /$ he is feeling bored." 


\section{Physical activity level}

Parents fill in questions from the Local and National Youth Health Monitor (LNMJ) [107] to specify the physical activity level of their child. They are asked to report how many days a week and how many minutes a day their child watches television, sits behind the computer, plays outside, engages in sports at a sports club, has gym or swimming classes at school, walks or bikes to school, and walks or bikes during leisure time during a normal week.

\section{Dietary behaviours}

Dietary behaviours are measured with 17 questions [108]; for the children, these are filled in by their parents. In addition to the adult questionnaire, which is similar to the LNMJ, the parents fill in extra questions about different types of beverages: water, diet beverages, and energy or sports drinks.

\section{Sleep}

Parents are asked to report the number of hours their child sleeps on week days and during the weekend, using 2 open questions. The quality of sleep is examined by means of 4 questions, such as "During the last month, my child woke up during the night" [109].

\section{Quality of life}

The Impact of Weight on Quality of Life (IWQOL)-Kids [110, 111] is used to measure their children's quality of life, using 27 items for the following scales: physical comfort ( 6 items), body esteem ( 9 items), social life ( 6 items), and family relations ( 6 items). The questions are answered on 5-point Likert scales from never (1) to always (5). An example of a statement on physical comfort is: "Because of my child's weight, she/he avoids using the stairs as much as possible."

\section{BMI}

$\mathrm{BMI}$ is recoded into BMI standardised for age and gender (i.e. BMI z-score) [14, 112]. Weight status will be recoded into different categories based on international cut-off values for overweight $\left(\mathrm{BMI} \geq 25 \mathrm{~kg} / \mathrm{m}^{2}\right)$ and obesity $\left(\mathrm{BMI} \geq 30 \mathrm{~kg} / \mathrm{m}^{2}\right)$ based on the $\mathrm{BMI}$ of adults aged 18 years and older [113].

The Children's Body Image Scale (CBIS) [114] is used to identify the discrepancy between the child's body image perception as reported by the child and by their parent. This is assessed with the question: "Which body picture is most like your own (child's) figure?" In addition, the discrepancy between the actual BMI and the perceived BMI category is assessed. The perceived BMI category is measured with 1 question using a 5-point Likert scale from much too light (1) to much too heavy (5): "How would you describe your child's weight?"

\section{Process evaluation questions}

The parents fill in the same process evaluation questions as the adults fill in. See description in the section on the questionnaire for adults. 


\section{Children's questionnaire}

\section{Home environment}

Children are asked whether they have certain devices available at home, like a television (in their own room), laptop, tablet, mobile phone, or bike. Furthermore, there is a question asking whether the child has been bullied or teased.

\section{Personality}

We use the TMCQ [97] to measure impulsivity from the child's point of view, with 13 questions. Questions such as "I make up my mind to do things all of a sudden" can be answered on a 5-point Likert scale (with options from almost never applicable (1) to almost always applicable (5)).

\section{Quality of motivation}

The children's enjoyment of physical activity is assessed using the Physical Activity Enjoyment Scale (PACES) [115], and the motivational mechanisms of their physical activity behaviour by the Behavioural Regulation of Physical Activity in Children (BRePAC) [Bogaards $L$ et al., unpublished observations] instrument, consisting of 16 and 18 items, respectively. Only the two contexts, in which children tend to be active, of the BRePAC are included in the questionnaire, viz. sporting outside school and playing outdoors. An example question from the PACES is: "When I am physically active I enjoy it"; one from the BRePAC is: "I participate in sports, but it is boring." This is measured on a 5-point Likert scale from totally disagree (1) to totally agree (5). Seven items $(2,3,5,7,12,13$ and 16) of the PACES are negatively formulated and they must be reversed before the total score can be calculated.

\section{Behaviour-specific cognitions}

Children's self-efficacy is measured with 13 items on playing outside and 8 items on eating fruit. These questions, with a 5-point Likert scale ranging from very difficult (1) to totally not difficult (5), are based on the questionnaire of previous study [116]. A question about playing outside is: "I think it is difficult to play outside on most days after school."

Attitude about eating fruit is measured by 7 questions. An example is: "Eating fruit is only necessary when I am sick." The attitude questions use a 5-point Likert scale ranging from totally disagree (1) to totally agree (5).

\section{Habit strength}

We use a shortened version of the Self-Report Habit Index (SRHI) [117] to measure habit strength behaviour to assess the history of repetition, automaticity and expressing identity. The 3 questions (with a 5-point Likert scale from totally disagree (1) to totally agree (5)) are for the behaviours: engaging in sports, playing outside and eating fruit. One example is "Playing outside is something I do without thinking."

\section{Physical activity level}

Children are asked whether they attend a sports club. They are asked what type of sports they play and how many days a week they spend on each type of sport. In addi- 
tion, they are asked how they perceive their physical activity level, on a 5-point Likert scale from very low (1) to very high (5), and how active they are compared with their peers, on a scale ranging from a lot less (1) to a lot more (5).

\section{Dietary behaviours}

Dietary pattern is measured by how children perceive their fruit consumption, with answering options from very low (1) to very high (5), and how much fruit they eat compared with their peers, from a lot less (1) to a lot more (5).

\section{Questionnaire for lifestyle coaches}

A questionnaire to assess competences is sent to all participating lifestyle coaches. This questionnaire includes items about their personality, work engagement and coaching competences. Personality is measured with the BFI [87]. For work engagement we use the 17-item Utrecht Work Engagement Scale (UWES) [118]. The statements have answering options on a 7-point scale from (0) never to (6) always/every day. An example is: "My job inspires me." The competences are assessed with questions derived from the published profile of lifestyle coaches with a degree from a university of professional education [119], supplemented with questions about flexible working attitude, process monitoring, networking skills, entrepreneurship and innovative work attitude, derived from the competence assessment instrument for the Dutch universities [120].

\section{Analyses}

The interviews, group interviews, observations and notes from the project team meetings and from the telephone meetings will be analysed with the Nvivo qualitative program. All interviews are audio-recorded and the several interview transcriptions are coded by two coders. Discrepancies between the two coders will be discussed until agreement is reached. The quantitative data will be analysed using SPSS version 21.0 on the basis of descriptives (i.e. means and frequencies), t-tests or, in case of skewed data distributions, non-parametric alternatives, and multivariate regressions. The absenteeism and dropout will be analysed using descriptives and logistic regression to test for selectivity. 


\section{DISCUSSION}

The purpose of this study protocol paper is to describe our study design to evaluate the implementation of the CooL intervention and to examine how the lifestyle coaches contribute to a healthier lifestyle of the participants. The added value of this study lies in the use of mixed methods (i.e. triangulation), which will increase the internal validity. This will give us information from different points of view, viz. those of the referrers, lifestyle coaches and participants. Furthermore, we combine selfreported data, observations and biomedical data. We are aware of the subjective view and potential prejudgements of the researchers, which we try to eliminate with these procedures.

Another strength of this study is its action-oriented approach, which helps us to implement and sustain the CooL intervention in the real-world setting. The observations of sessions and the interaction between the lifestyle coaches, the intermediaries and the research team enable the adjustment of the intervention programme and its implementation process during the intervention period. Furthermore, the lifestyle coaches can initiate discussions to change the programme based on their practical experiences gained during the implementation of the intervention. However, these programme changes make it more difficult to evaluate the intervention and its implementation process.

The use of one-group pre-post design makes us unable to draw definite conclusions of the causal relationship between the CooL intervention and the lifestyle changes achieved by participants over time. In contrast, the purpose of this study is primarily to answer the 'how' and 'why' questions, e.g. "How have the participants changed their lifestyle and how did they perceive the intervention to affect this?" We try to increase the external validity to combine the information from the different regions, so that we can distinguish important theoretical similarities.

The first results of the study are expected to be available at the end of 2017. 


\section{APPENDIX 1 - INCLUSION CRITERIA}

\section{Adults}

- $\quad \geq 25 \mathrm{BMI}<30$ :

- 10 -year risk of death from (cardiovascular diseases or type II diabetes) risk factors $>5 \%$ or,

- Impaired fasting glucose or,

- Comorbidity:

- $\quad$ Type II Diabetes

- CVD

- Sleep apnea

- Osteoarthritis

- $\quad \mathrm{BMI} \geq 30$

\section{Children}

- 4 to 10 years old:

- $\quad \geq 25 \mathrm{BMI}<30$ with a high risk of type II diabetes, which means having at least two risk factors:

- $\quad 1$ st or 2 nd degree relative with type II diabetes

- $\quad$ Ethnicity (non-Western descent)

- Signs of insulin resistance in children:

- Dlipidemia

- Low birth weight

- Acanthosis nigricans

- Polycystic ovary syndrome (signs of excessive testosterone production: irregular periods, excessive hair growth, excessive acne)

- Hypertension

- Gestational diabetes of the mother during pregnancy

- $\quad \mathrm{BMI} \geq 30$

- 10 years or older:

- $\quad \geq 25 \mathrm{BMI}<30$ with a high risk of type Il diabetes, as determined by blood tests.

- Triglycerides: $\geq 1.7 \mathrm{mmol} / \mathrm{L}$

- HDL cholesterol: < $1.03 \mathrm{mmol} / \mathrm{L}$ for boys; $<1.29 \mathrm{mmol} / \mathrm{L}$ for girls

- Fasting glucose: $\geq 5.6 \mathrm{mmol} / \mathrm{L}$

- $\quad \mathrm{BMI} \geq 30$

- $\quad \geq 35 \mathrm{BMI}>40$ : referral to the lifestyle coach has to be discussed with the pediatrician. 


$$
\text { (q) }
$$




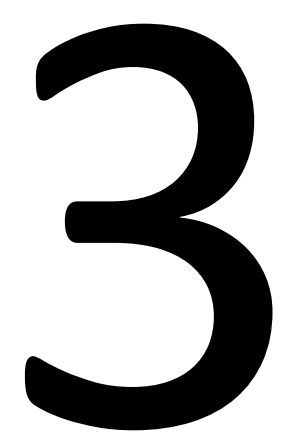

\title{
Coaching on Lifestyle (CooL) intervention: the lifestyle coach as a linchpin?
}

\author{
Celeste E van Rinsum, Sanne MPL Gerards, Geert M Rutten, len AM van de Goor,
} Stef PJ Kremers

Coaching op Leefstijl (CooL) interventie: de leefstijlcoach als spin in het web? Tijdschrift voor gezondheidswetenschappen, 2018; 96(5), 189-193 (published in Dutch) 
$42 \mid$ Chapter 3

\begin{abstract}
The Coaching on Lifestyle intervention ( $\mathrm{CooL}$ ) is a combined lifestyle intervention in the Netherlands, in which professional lifestyle coaches counsel adults and children (and/or their parents) who are obese or at high risk of obesity. The CooL intervention consists of group and individual sessions addressing the topics of physical activity, dietary behaviours, sleep and stress. CooL has shown to have potential in coaching adult participants towards sustained behavioural change and in connecting professionals in the care sector and the public sector. For children, no definite conclusions can be drawn regarding the potential added value of the lifestyle coach.
\end{abstract}


Half of all adults in the Netherlands, and $12 \%$ of all children, are currently overweight or obese [4]. Overweight and obesity are caused by an imbalance between energy uptake and energy expenditure, resulting from complex behaviours, with many underlying factors [8]. Combined lifestyle interventions (CLIs) have been found to effectively change the dietary and physical activity behaviours of people with obesity [121, 122]. CLIs involve supporting people in changing their behaviour in order to reduce their energy intake and increase their level of physical activity. According to the Dutch care guidelines on obesity [14], a CLI is the treatment of first choice for children and adults with obesity.

\section{THE COACHING ON LIFESTYLE INTERVENTION}

Studies of the implementation of CLIs, like BeweegKuur, SLIMMER, MetSLIM, Lifestyle Triple $\mathrm{P}$ and $\mathrm{COACH}[38,50,51,54,57]$ have been used in the development of the Coaching on Lifestyle ( $\mathrm{CoOL}$ ) intervention. The CooL intervention gives professional lifestyle coaches, who have been trained to help people take control of their own health, the central role in the network of care and prevention. The objective of the CooL programme is to gradually improve people's life habits, in order to achieve a sustainable change in their lifestyle, the ultimate goal being to reduce their weight-related health risks and improve their quality of life. A lifestyle coach will be called upon when lifestyle counselling by providers like general practice nurses is insufficient, and more intensive guidance is deemed to be required.

The CooL programme involves individual and group sessions offered by a lifestyle coach. All lifestyle coaches involved in CooL have had a postgraduate training at AVLEG (the Dutch Academy for Lifestyle and Health). Lifestyle coaches have been trained in all aspects of lifestyle and coaching. They focus on participants' personal goals and their counselling covers all relevant lifestyle themes. In the context of the programme, they counsel adults and children from the age of four years with overweight or obesity. The programme comes in three versions: one for children (and their parents), one for adolescents and one for adults. Together with the participants, the lifestyle coach assesses the underlying factors and causes of their unhealthy lifestyle, after which the participants enter a programme of treatment. Major themes include dietary behaviours, physical activity, sleep and stress management. If participants who have completed the basic programme are found to be still in need of further counselling, they can enter one of the two supplementary programmes (i.e. the relapse prevention programme and additional programme).

The present paper reports on a research study we undertook to evaluate the process of implementing the CooL programme. More detailed information on the intervention and research components can be found in our research protocol, which has been published elsewhere [123]. A more detailed discussion of research findings has also been published in a research report (in Dutch) [78]*.

* https://www.maastrichtuniversity.nl/nl/nieuws/leefstijlcoach-motiveert-mensen-met-overgewicht-om-gezonder-te-leven 


\section{RESEARCH}

The aim of our research project was to examine whether lifestyle coaches represent a valuable addition to the care network around people with increased weight-related health risks. The research project was basically action-oriented, meaning that our observations of the programme as it is used in practice were evaluated in light of the available scientific knowledge, which could then be used to adjust the programme's content or process. In the process, we have tried to identify opportunities and barriers for the programme, which could be used to improve it. Data for the research project were collected between 1 April 2014 and 1 April 2017, while monitoring the implementation of the Cool programme. To this end, we engaged upon a quantitative process evaluation (using questionnaires and data on the numbers of people entering and dropping out of the programme), as well as a qualitative process evaluation (using interviews and observations) and an assessment of lifestyle changes among the participants (using questionnaires and anthropometric measurements carried out by professional staff). The qualitative part of the project studied it from the perspective of the professionals involved, as well as that of the participants. The main research question was how the implementation of the CooL intervention was going and how the lifestyle coaches contributed to the process of guiding the participants towards a healthier lifestyle.

\section{RESULTS}

The process evaluation study found that referrals of adults to the programme $(n=494)$ were slow to start. The primary referrers (practice nurses and staff at youth health care services) were not always very aware of the programme, and some of the lifestyle coaches lacked the 'entrepreneurial' skills to effectively draw their attention to it. Active referrers were found to be characterised by a greater personal interest in the problems of overweight.

Once participants had entered the programme, drop-out rates were low (21\% among the children and adolescents and $18 \%$ among the adults). Many of the participants had a low educational level (35\% of the adults; $40 \%$ of the parents of the child participants). The participants expressed favourable opinions about the various programme components. They gave the CooL programme as a whole a rating of 8 out of 10 (adults: 8.6; parents: 8.5 ; children: 7.8 ). Many of the participants indicated that they felt a need for refresher meetings. These meetings have now been developed and made available.

The participants reported that since taking part in the CooL programme, they had started to exercise more, spend less time sitting and eat a healthier diet than before. Their extrinsic motivation had diminished, while their intrinsic motivation for healthier dietary and exercise behaviours had been strengthened. Their reported quality of life had improved significantly. The adult participants had lost an average of $2.2 \mathrm{~kg}$ of body weight after taking part in the programme, a weight loss that was largely maintained up to two years after the baseline assessment. 
Although a large proportion of the participants failed to achieve the desirable $5 \%$ weight reduction, our findings showed that the risk of relapse and weight gain was lower than with interventions that focus more on short-term weight loss [124]. The principles underlying the $\mathrm{CooL}$ intervention are based on solid arguments for focusing on small steps, sustainable change and a holistic approach to the concept of health. Sustained behavioural change among the target group of people with elevated weight-related health risks is very difficult to achieve. Many of the participants had already made several failed attempts at losing weight. One frequent pitfall for health care providers is that of focusing primarily on a participant's weight and on overly drastic changes to behavioural patterns in order to achieve weight loss. This inevitably leads to failures, controlled motivation and, in the longer term, weight gains. Our results show that the CooL intervention is using the right approach in this respect.

Our findings among the children and adolescents $(n=192)$ showed favourable changes regarding some of the indicators of quality of life, dietary behaviour and parenting. In the short term, we found a reduction of BMI z-scores. No long-term changes were found as regards exercise, motivation or weight. However, the number of participants was small, making it difficult to draw definitive conclusions from these results. What is obvious is that it is difficult to ensure that children and adolescents are referred to the programme, and to achieve behavioural change among children with frequently complex family situations and deep-rooted routines.

Our study found that some of the lifestyle coaches did not manage to effectively make health care providers aware of the programme. It is a well-known fact that it requires a certain start-up period to make referrers take an interest in an innovative intervention, support it and know how to refer to it [125]. In the case of CooL we observed that referrals, especially of adults, went more smoothly at the end of the pilot period than at first. This means that future efforts need to take this start-up period for the implementation of innovations into account. It can be regarded as the period required to try out the innovation, to inform and establish contacts with referrers and lifestyle coaches, to find out each other's expertise and to learn from positive and negative experiences.

As more people are being referred to the intervention, the lifestyle coaches will be enabled to optimise the composition of the groups. We found that both coaches and participants would prefer a more homogeneous group composition (especially in terms of age and cognitive skills). If groups are more homogeneous, the lifestyle coaches can tailor their message to the whole group, and increase the chances that participants can empathise with each other's experiences.

\section{Role of the lifestyle coach}

The role of the lifestyle coach as a linchpin should be made better use of. This is part of the strength of CooL, as practical experience shows that it is hard to bridge the gap between care and prevention in existing networks. The CooL pilot project insufficiently managed to bridge this gap. We therefore recommend that the lifestyle coaches (including their professional association) should draw attention to the opportunities that 
are being missed and the need for cooperation. The coaches rated their own entrepreneurial skills in this respect as rather low, and also considered these to be the least important skills they needed for their work. Hence, their training needs to focus not only on professional development but also on greater awareness and knowledge acquisition.

The degree to which the lifestyle coaches assume a central position in the network greatly determines the referral rates, as well as the coaches' ability to offer appropriate care to the participants. The regional or national organisational structures in which the lifestyle coaches work should be organised in such a way as to safeguard the quality of their professional activities. On the other hand, the success of the coaches' work depends on the context in which they function. Contexts in which the prevention pyramid is more fully developed (which means that effective universal, selective, indicated prevention and treatment is being offered) [126] present more opportunities for a suitable implementation of the role of lifestyle coaches. An increasing number of regions in the Netherlands show a favourable trend towards high-quality implementation of the entire prevention pyramid. This development is creating chances for a more effective integration of lifestyle coaching in the chain of health care. Lifestyle coaches are less likely to find themselves in an isolated position if they work in a context where and general practitioners, public health services and municipal authorities give priority to and support the links between care and prevention. Hence, the integrated approach to the problem of overweight can be more effectively implemented by coordinating the activities of key parties [127].

\section{RECOMMENDATIONS}

Table 1 offers some recommendations we can make on the basis of our research findings, to improve the way the lifestyle coaches function and the contents of the CooL programme. 
Table 1: Recommendations for the way lifestyle coaches function, the content of the CooL programme and the organisations implementing the intervention

\begin{tabular}{ll}
\hline Theme & Recommendations \\
\hline Lifestyle coaches & The encouraging attitude of the lifestyle coaches and their focus on improving \\
the quality of participants' motivation to change their behaviour should be main- \\
tained. \\
-
\end{tabular}

- Participants with complex problems should be referred sooner to other relevant professionals, who can provide the appropriate care for their problems. This is true for adults, but especially also for children in complex family situations.

- The quality of the lifestyle coaches' professional activities should be ensured. One aspect of this is safeguarding the quality of their training courses (e.g. by means of inspections and certification), while another is the development of a transparent profile of professional competences and a professional code of conduct.

- Peer feedback and supervision meetings should be systematically incorporated in the CooL programme, for the promotion of contacts, quality assurance and the exchange of ideas.

Programme

- The programme should be further developed regarding interdisciplinary collaboration, the coaches' competences (especially regarding referrals, entrepreneurship and counselling children and their parents), professionalisation of the organisational structure and further development of the relapse prevention programme.

- The principles that make the programme effective should be clearly described and communicated to the professionals in the field, to make them widely available. One of the ways to ensure this in the Dutch context is through certification by the Centrum Gezond Leven.

- The programme should pay more attention to the target group of people with a migrant background, preferably by developing an intervention specifically adapted to their cultural characteristics.

- The intervention for children should be improved by also offering them the option of individual counselling or fewer group sessions.

- Consideration should be given to the possibility of extending the role of the lifestyle coaches to include the selective prevention of overweight among children (e.g. based on socioeconomic status) or for indicated prevention specifically based on a child's overweight status, in order to increase the coaches' reach.

Organisation - The process-related preparations for the programme, including clear conditions, rooms, referral structure and the record-keeping and expenses system, should be completed before professionals and lifestyle coaches can optimise their collaboration. Extra preparatory time will need to be made available in regions with a fragmented network. There has to be sufficient time for efforts to inform referrers and lifestyle coaches and bring them into contact with each other, as well as for them to find out each other's expertise and to experiment in order to learn from each other's experiences.

- General practitioners, public health services and local authorities should give priority to and offer support for connecting local care and prevention services, as well as relevant national organisations at the national level. 


\section{CONCLUSION}

Our research findings show that lifestyle coaches can fulfil an important role in connecting the various links in the chain of professionals addressing the problem of overweight in the Netherlands, especially regarding the adult target group. Lifestyle coaches provide easily accessible guidance towards sustainable behavioural change, as well as connecting professionals in the care and public domains, and referring participants to other relevant services. As regards the target group of children and adolescents, our findings do not as yet allow definitive conclusions to be drawn regarding the added value offered by integrating lifestyle coaches in the chain of care, as our sample size was too small, and behavioural change proved to be difficult to achieve within the study period in the often complex family situations.

The CooL programme offers useful opportunities to sustainably incorporate lifestyle coaching in the integrated approach to obesity. We have identified a number of starting points for further optimisation of the programme. The programme should be further developed as regards interdisciplinary collaboration, the coaches' competences (especially those concerning referrals, 'entrepreneurship', and counselling children and their parents), creating a more professional organisation (including quality assurance by means of peer feedback and supervision) and further elaboration of the relapse prevention programme. In addition, it would seem advisable not to use weight loss as the most important outcome measure, but to focus instead on lifestyle-related indicators. As regards the possible extended implementation on a national scale, the pilot project has shown once again that it is important to take enough time to inform referrers and lifestyle coaches, and bring them into contact with each other, so they can find out each other's expertise and learn from each other's positive and negative experiences. In the coming years, the Dutch prevention and care situation is expected to develop in such a way that lifestyle coaches will be able to assume a central position, together with other key parties, in order to optimise the integrated approach to combating obesity. 
3 


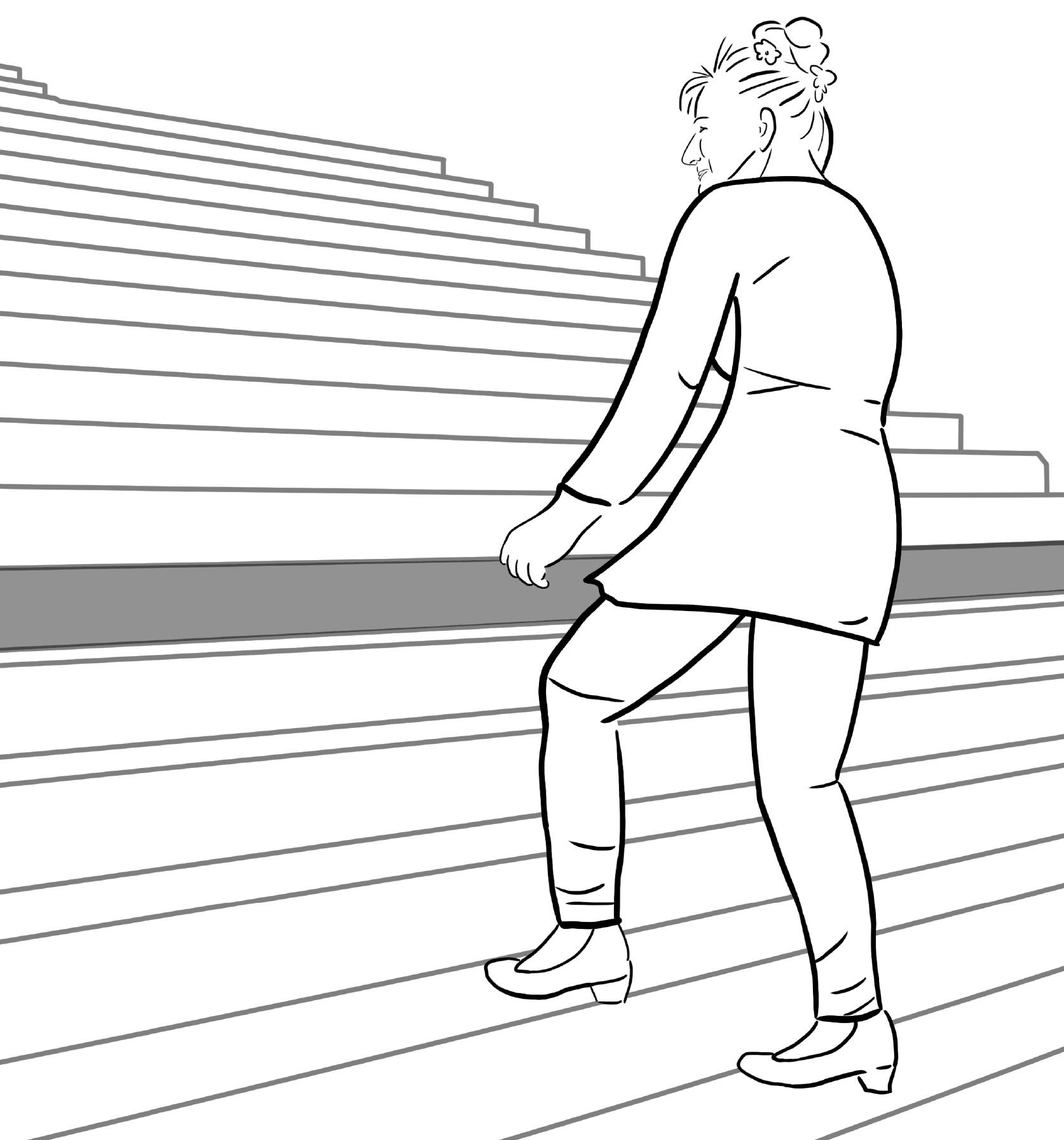




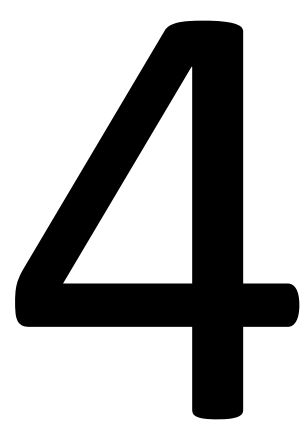

\section{The Coaching on Lifestyle (CooL) intervention for overweight and obesity: a longitudinal study into participants' lifestyle changes}

Celeste E van Rinsum, Sanne MPL Gerards, Geert M Rutten, Nicole ML Philippens,

Ester MJ Janssen, Bjorn Winkens, len AM van de Goor, Stef PJ Kremers

International Journal of Environmental Research and Public Health, 2018; 15(4), 680 
$52 \mid$ Chapter 4

\begin{abstract}
Combined lifestyle interventions (CLIs) can be effective in reducing weight and improving lifestyle-related behaviours but it is unclear how CLIs can best be implemented in practice in order to achieve sustained lifestyle changes. The Coaching on Lifestyle programme ( $\mathrm{CooL})$ is a $\mathrm{CLI}$ in the Netherlands, in which professional lifestyle coaches counsel adults and children (and/or their parents) who are obese or at high risk of obesity to achieve a sustained healthier lifestyle. The CooL intervention consists of group and individual sessions addressing the topics of physical activity, dietary behaviours, sleep and stress. Our longitudinal one-group pre-post study aimed to identify lifestyle changes among participants (adults, children and their parents) at 8 and 18 months after initiation. We assessed constructs ranging from motivation and behaviour-specific cognitions to behaviours and health outcomes. Positive and sustained changes among adults were found regarding perceived autonomy, motivation, perceived barriers, lifestyle behaviours, quality of life and weight. Among children and their parents, few improvements were found regarding behaviours and quality of life. CooL has been successful in coaching adult participants towards sustained behavioural change during the intervention period. Mixed results and smaller effect sizes were found for children and their parents.
\end{abstract}


Longitudinal lifestyle changes of CooL participants $\mid 53$

\section{INTRODUCTION}

Worldwide, the prevalence of overweight and obesity is still increasing [1,2]. In The Netherlands, $50.2 \%$ of the adult population are overweight, $14.5 \%$ of whom are obese [4]. The number of children with overweight and obesity in particular has rapidly increased in recent decades [5]. Currently, $13.4 \%$ ofthe children in The Netherlands are overweight and $2.7 \%$ are obese. Overweight is caused by an imbalance between energy intake and energy expenditure [79]. Primary factors that may lead to a chronically disturbed energy balance include insufficient levels of physical activity, too much sedentary behaviour [9] and too much high-energy dietary intake [10]. This imbalance can also be influenced by sleep, since sleep deprivation may lead to a decrease in physical activity and an increase in energy intake due to disturbed hormone levels [11, 12].

Combined lifestyle interventions (CLIs) support people who are overweight or obese in initiating and maintaining healthier lifestyle behaviours, by increasing physical activity and improving dietary behaviours. CLIs include physical activity, dietary and behavioural components [45] and are typically carried out by general practitioners, practice nurses, physiotherapists, psychologists and/or dieticians [46]. These interventions have been shown to be effective in terms of weight reduction and health improvements compared with standard care or drug treatment $[45,58]$. Lifestyle effects of CLIs and other types of weight loss interventions, however, have been shown to be rather short-term; relapses are common in long-term weight loss [69].

The main causes of relapse are that it is not easy to incorporate sustainable lifestyle changes into daily behaviour patterns and that intervention success depends heavily on the quality and quantity of external stimuli from professionals. Motivation, as well as well-being and resilience, are strong predictors of sustained healthy lifestyle behaviours and weight loss [69]. In addition, previous CLI studies identified undesirable implementation issues: lack of multidisciplinary collaboration, insufficient coaching skills among primary care professionals, insufficient time to optimize coaching and high intervention costs [35, 72].

In comparison to coaching adult participants, supporting lifestyle changes among overweight children may even be more complex. Since parents play a key role in determining their children's lifestyle behaviour [74], both parents as well as their children are advised to participate in CLIs. However, parents often do not see their child's weight as a cause for concern [128], which decreases the likelihood that these families will participate in CLIs. In addition, the interplay between multiple contextual family-based factors (parenting, broken families, lack of resources) may undermine potential intervention effects [74].

Insights into implementation issues obtained from previous intervention studies on both adults and children in The Netherlands [35, 38, 54, 86] have led to the design of the Coaching on Lifestyle ( $\mathrm{CooL}$ ) intervention for people who are overweight or obese [123]. The present paper discusses the results of the CooL intervention in a longitudinal one-group pre-post study using validated questionnaires and objective data. The study 
investigated the motivational, behavioural, quality of life and weight changes achieved by the CooL participants (i.e. adults and children) during the intervention period and in the longer term.

\section{METHODS}

A detailed description of the study protocol can be found elsewhere [123]. Below, we providemore details about the CooL intervention, followed by the study design and methods. This study was registered in a trial register (Dutch Trial Register: NTR6208) and it was exempt from review by a research ethics committee (METC 14-5-021), as it does not fall within the scope of the Dutch Medical Research Involving Human Subjects Act (Central Committee on Research Involving Human Subjects (CCMO), 2015).

\section{Cool: the Coaching on Lifestyle intervention}

CooL is a CLI in The Netherlands, in which professional lifestyle coaches counsel adults and children (and/or their parents) who are obese or at high risk of obesity to help them achieve a sustained healthier lifestyle. The lifestyle coaches who took part in the present study had completed a post-graduate lifestyle coaching education programme. Health coaching or lifestyle coaching, although relatively new in both practice and research [129], is a promising approach to achieve positive behavioural and health outcome changes [130-132].

Lifestyle coaching entails a client-oriented approach. The starting-point is the current lifestyle and the associated healthy and unhealthy habits of the participant. During the process of changing their lifestyle, the participants remain in charge of their intended (behavioural) goals. Unhealthy behaviours (mainly concerning physical activity, nutrition, sleep, stress management and relaxation) are identified and throughout the coaching sessions each participant is stimulated to replace unhealthy routines by healthier alternatives. The lifestyle coaches support participants in their change process by helping them to identify their unhealthy as well as their healthy habits and underlying processes. Furthermore, they stimulate the participants to take responsibility for their own behaviour and to identify challenging but achievable action steps for each of the above behaviours in order to set feasible and sustainable goals that will improve their long-term health. The lifestyle coaches' role includes listening, supporting, motivating and providing feedback to the participants as well as providing background information on health topics and behavioural change [131].

The CooL programme consists of 8 group sessions and 4 to a maximum of 10 individual sessions targeting physical activity, dietary behaviours, sleep and stress. The programme as a whole lasts approximately 8 to 10 months. Three different basic programmes focus on three age groups: primary school children (aged 4 to 12 years), adolescents (aged 12 to 18 years) and adults (aged 18 years and older). In the children's and adolescents' programmes, parents participate together with their child. 
Longitudinal lifestyle changes of CooL participants $\mid 55$

Various evidence-based techniques and approaches are incorporated in the basic programmes (see Appendix A, Table A1), which are similar to other interventions [133, 134]. In addition to the basic programmes, coaches can, if required, assess the needs of each participant and the appropriateness of being included in the relapse prevention intervention (group and individual sessions) or the additional intervention (individual sessions). These supplementary programmes were set up to prevent relapse and to maintain the healthy behaviours in the longer term.

\section{Design and study population}

In this one-group pre-post study, the lifestyle changes of the CooL participants were monitored over time. The target population of this intervention consisted of Dutch-speaking individuals living in The Netherlands, aged 4 years and older, who were obese (BMI 30) or at high risk of obesity (i.e. were overweight (BMI 25) and at increased risk of cardiovascular diseases or type 2 diabetes mellitus) [14, 40,41]. The exclusion criterion was being unable to function in a group (e.g. because of behavioural disorders).

Adults were often referred by their general practitioner or practice nurse in three different regions (Oosterhout and its region, Parkstad and Tilburg) within two provinces of the southern part of The Netherlands. The recruitment of children mostly took place via referral by Youth Health Care (YHC) professionals, schools (such as those taking part in the 'Healthy Elementary School of the Future' programme [84]), pedagogical workers and paediatricians in two regions ('s-Hertogenbosch and Breda) in one of the same two provinces. Participants could also sign up for the intervention themselves. These participants were instructed to consult their health care provider (e.g. general practitioner) to check whether they met the inclusion criteria. All participants were asked to sign an informed consent form when they started in the study. Both parents were asked to sign for informed consent and in case of adolescents (12-18 years), also the child had to sign an informed consent form.

\section{Measurements}

\section{Questionnaires}

The participants completed three questionnaires: at baseline (T0); immediately after the intervention, which was approximately 8 to 10 months after baseline (T1); and 18 months after baseline (T2). The questionnaires were adapted to the three different groups: the older children (aged 10 years and older) and their parents both filled in their own questionnaires, while the adult participants (18 years and older) received a different version of the questionnaire. Children younger than 10 years were considered too young to fill out a questionnaire. The constructs measured in the questionnaires were based on the theoretical framework of this study [123], which consists of psychological needs, parenting, motivation, behaviour-specific cognitions (e.g. barriers) and energy balance-related behaviours, which were assumed to produce the health outcomes (i.e. quality of life and $\mathrm{BMI}$ ). Where possible, validated questionnaires were used (see Appendix $A$, Tables $A 2$ and $A 3$ for an overview and reliability indices). The attendance lists, 
filled out by the lifestyle coaches, provided insights in the attendance rates and dropout.

\section{Body weight}

Body mass index (BMI) of adults was objectively measured by the practice nurses, general practitioner and/or researchers. When objective weight data were missing (14\%), they were replaced by self-reported weight. The weight status was classified into five categories according to international guidelines [7]: normal weight (18.5 BMI $24.99 \mathrm{~kg} /$ $\mathrm{m}^{2}$ ), overweight (BMI $\left.25 \mathrm{~kg} / \mathrm{m}^{2}\right)$, obesity (BMI $\left.30 \mathrm{~kg} / \mathrm{m}^{2}\right)$, severe obesity (BMI $35 \mathrm{~kg} /$ $\mathrm{m}^{2}$ ) and morbid obesity (BMI $40 \mathrm{~kg} / \mathrm{m}^{2}$ ).

For children, we used objectively measured BMI, as assessed by $\mathrm{YHC}$ professionals, lifestyle coaches and/or researchers. When objective data were missing, we imputed this with the child's weight as reported by the parent. The data were converted to BMI standardized for age and gender (i.e. BMI z-score) using the Dutch reference population $[14,112]$. Weight status was categorized using international cut-off values [113].

\section{Statistical analyses}

Numerical variables are presented as means (SD), while the number of participants (\%) is used for categorical ones. Linear mixed model analysis was used to assess the changes over time, using an unstructured or compound symmetric (where appropriate) covariance structure for repeated measures. In addition, a random intercept at coach level was included to take the nesting of participants within coaches into account. The longitudinal trend was assessed by including time (categorical; T0, T1 and T2) in the fixed part of the model and was corrected for demographic characteristics such as age, gender, living situation, occupational status, highest completed education and BMI or BMIz (if BMI was not the outcome), if these characteristics were related to missing outcome values. All data from participants who did not drop out before the first group session were included in these analyses. Longitudinal effects were presented as corrected estimated mean difference in change scores from baseline, together with their corresponding $95 \%$ confidence intervals and $p$-values.

Standardized effect sizes were also included, which were defined as (mean at T1 or T2mean at T0)/standard deviation at TO. The effect sizes were categorized in accordance with Lipsey's guidelines [135]: small effect (0-0.32), medium effect $(0.33-0.55)$ and large effect (higher than 0.56). As a result of the large amount of outcome measures, we aimed to summarize the observed changes in a graph for illustrative purposes. For the adult population, the average effect sizes regarding psychological needs, motivation and barriers ('motivation'), physical activity, dietary behaviours ('behaviour'), weight and quality of life were plotted in a graph. For the children and adolescents, the average effect sizes of the general parenting and parenting practices were plotted together ('parenting'), the motivation and behaviour-specific cognitions ('motivation') and the physical activity, dietary and sleeping behaviours ('behaviour').

All analyses were performed using IBM SPSS Statistics for Windows, version 23.0 [136]. A two-sided $p$-value 0.05 was considered statistically significant. 


\section{RESULTS}

\section{Characteristics of participating adults}

The pre-test questionnaire was filled in by 138 participating adults between 20th of May 2014 and 15th of February 2016. The adult participants consisted of an evenly divided group of men and women, whose mean age was 55.1 years (SD 10.1; see Table 1). In total, $86 \%$ of the participants were obese. Almost all (95\%) participants had been born in The Netherlands, more than three quarters of participants had a low or intermediate educational level, more than half the participants did not have a steady job and most of them (71\%) were living with a partner.

\section{Characteristics of participating children}

Demographic characteristics of 31 children and 10 adolescents who participated (referred to below as 'children') are presented in Table 1. Children were nearly evenly divided in terms of gender. The average BMI z-score of children was 2.4 (SD 0.4). A large majority of the children who participated were obese (93\%). Most parents were overweight too. The mean BMI of the mothers and fathers was 30.4 (SD 6.5) and 27.3 (SD 4.6), respectively. Almost all children were born in The Netherlands (97\%) but $23 \%$ had a Turkish or Moroccan migration background. The majority of the mothers had an intermediate or lower educational level (73\%) and the majority of the fathers had a low educational level (57\%). In more than half of the families both parents had a job and most parents were living together. On average the families consisted of four persons.

\section{Drop-out and attendance}

Among the adult participants, $13 \%(n=18)$ dropped out of the intervention. There was a loss to follow-up regarding measured weight of $20 \%$ at T1 and $23 \%$ at T2 (included $n$ $=110$ and $n=106$, respectively). Regarding the questionnaires, the loss to follow-up was $33 \%$ at T1 $(n=93)$ and $45 \%$ at T2 $(n=76)$. Of the demographic characteristics, only gender was significantly different in the adult drop-out group compared with the rest, as more women than men dropped out $(p=0.023)$. Among the children, $7 \%(n=3)$ dropped out of the intervention. The loss to follow-up regarding measured weight was $22 \%$ at T1 and $29 \%$ at T2 ( $n=32$ and $n=29$, respectively), while loss to follow-up regarding the questionnaires was $41 \%$ at T1 and $49 \%$ at T2 ( $n=24$ and $n=21$, respectively). For the children, there were no significant demographic predictors of loss to follow-up.

The total amount of sessions in the protocol for adults consisted of 8 groups sessions and 3.5 individual hours. The adults participated on average in 5.9 (1.9) group sessions and had $3.2(0.7) \mathrm{h}$ of individual counselling from their lifestyle coach. Their average total intervention period was 219.9 (76.0) days. The protocol for children and/or their parents consisted of 8 group sessions and $7 \mathrm{~h}$ of individual counselling. The children and/or their parents attended on average in 4.6 (2.6), group sessions and they had 5.4 (1.4) $\mathrm{h}$ of individual sessions. They participated in the intervention for a period 283.0 (103.0) days. 
$58 \mid$ Chapter 4

Table 1: Demographics and weight-related characteristics of the participants

\begin{tabular}{|c|c|c|c|c|}
\hline \multirow[b]{2}{*}{ Variable } & \multicolumn{2}{|c|}{ Adult participants ( $n=138)$} & \multicolumn{2}{|c|}{$\begin{array}{c}\text { Child }(n=31) \text { and adolescent }(n=10) \\
\text { participants }\end{array}$} \\
\hline & Mean (SD) & N (\%) & Mean (SD) & N (\%) \\
\hline \multicolumn{5}{|l|}{ Gender } \\
\hline Male & & $66(47.8)$ & & $18(43.9)$ \\
\hline Female & & $72(52.2)$ & & $23(56.1)$ \\
\hline Age & $55.1(10.1)$ & & $10.2(3.5)$ & \\
\hline $\mathrm{BMI} / \mathrm{BMIz}^{1}$ & $36.0(5.8)$ & & $2.4(0.4)$ & \\
\hline Missing & & 5 & & \\
\hline \multicolumn{5}{|l|}{ Weight status } \\
\hline Overweight & & $18(13.5)$ & & $3(7.3)$ \\
\hline Obesity & & $44(33.1)$ & & $23(56.1)$ \\
\hline Severe obesity & & $45(33.8)$ & & $11(26.8)$ \\
\hline Morbid obesity & & $26(19.5)$ & & $4(9.8)$ \\
\hline Missing & & 5 & & \\
\hline BMI of mother ${ }^{1}$ & & & $30.4(6.5)$ & \\
\hline Missing & & & & 7 \\
\hline BMI of father ${ }^{1}$ & & & $27.3(4.6)$ & \\
\hline Missing & & & & 14 \\
\hline \multicolumn{5}{|l|}{ Country of birth } \\
\hline Netherlands & & $125(95.4)$ & & $35(97.2)$ \\
\hline Other & & $6(4.6)$ & & $1(2.8)$ \\
\hline Missing & & 7 & & 5 \\
\hline \multicolumn{5}{|l|}{ Ethnicity ${ }^{2}$} \\
\hline Dutch & & & & $29(74.4)$ \\
\hline Turkish & & & & $2(5.1)$ \\
\hline Moroccan & & & & $8(20.5)$ \\
\hline Missing & & & & 2 \\
\hline \multicolumn{5}{|c|}{ Educational level of adults or of mother } \\
\hline Low & & $50(38.2)$ & & $11(29.7)$ \\
\hline Intermediate & & $53(40.5)$ & & $16(43.2)$ \\
\hline High & & $28(21.4)$ & & $10(27.0)$ \\
\hline Missing & & 7 & & 4 \\
\hline \multicolumn{5}{|c|}{ Educational level of father } \\
\hline Low & & & & $21(56.8)$ \\
\hline Intermediate & & & & $9(24.3)$ \\
\hline High & & & & $7(18.9)$ \\
\hline Missing & & & & 4 \\
\hline \multicolumn{5}{|c|}{ Working situation (of parents) } \\
\hline Not in work & & $67(51.1)$ & & $6(15.4)$ \\
\hline One parent in work & & & & $13(33.3)$ \\
\hline
\end{tabular}




\begin{tabular}{|c|c|c|c|c|}
\hline \multirow[b]{2}{*}{ Variable } & \multicolumn{2}{|c|}{ Adult participants ( $n=138$ ) } & \multicolumn{2}{|c|}{$\begin{array}{c}\text { Child }(n=31) \text { and adolescent }(n=10) \\
\text { participants }\end{array}$} \\
\hline & Mean (SD) & $\mathrm{N}(\%)$ & Mean (SD) & $\mathrm{N}(\%)$ \\
\hline In work & & 64 (48.9) & & $20(51.3)$ \\
\hline Missing & & 7 & & 2 \\
\hline \multicolumn{5}{|c|}{ Living situation (of parents) } \\
\hline Living alone & & $38(28.8)$ & & 7 (17.9) \\
\hline Living together & & $94(71.2)$ & & $32(82.1)$ \\
\hline Missing & & 6 & & 2 \\
\hline Family size & & & $4.2(1.1)$ & \\
\hline Missing & & & & 2 \\
\hline
\end{tabular}

Notes: $\mathrm{SD}=$ standard deviation, $\mathrm{N}=$ number of participants; ${ }^{1}$ in $\mathrm{kg} / \mathrm{m}^{2}$ or standardized $\mathrm{BMI},{ }^{2}$ based on the children's and parents' country of birth.

\section{Changes among the adult participants}

Figure 1 offers a visual summary of all the changes among the adult participants regarding motivation, behaviours, quality of life and weight. A positive change in effect size corresponds to a change in a healthier direction. All four components were increased at $\mathrm{T} 1$, followed by a slight decrease (quality of motivation, behaviour and weight) or further increase (quality of life) at T2. All aggregated effect sizes can be categorized as small (0-0.32).

\section{Psychological needs}

Perceived autonomy increased significantly, with a small effect size, after the intervention, by 0.2 points on a 5-point Likert scale, which was maintained in the longer term at T2 with a medium effect size (Table 2). This means that the participants reported a higher sense of volition and willingness to engage in physical activity, compared to baseline.

\section{Motivation for physical activity and healthy diet}

External motivational regulation had decreased significantly and both integrated regulation and intrinsic motivation had increased significantly at post-test. This applies to the motivation for physical activity and that for a healthy diet, with some exceptions at T2. Introjected regulation for healthy diet had decreased significantly at the two follow-up moments. Overall, participants were more autonomously motivated (e.g. exercising because it is fun or eating a healthy diet because it is part of who you are) after the intervention, with small to medium effect sizes and had less controlled motivation (exercising because others say you should) for physical activity at T2 and for healthy diet at the post-test.

\section{Behaviour-specific barriers}

Participants perceived significantly fewer barriers, such as lack of time, lack of good equipment or lack of discipline, to being physically active and to eating a healthy diet at $\mathrm{T} 1$ and $\mathrm{T} 2$ compared with the pre-test, with medium effect sizes. 


\section{Energy balance-related behaviours}

Participants reported sitting significantly less after the intervention compared with the pre-test (-123.2 minutes a day), with a large effect size. They also engaged in more moderate-intensity and vigorous-intensity activity (medium effect size), with the largest increase at T2. Changes in dietary behaviour were in the desired direction. Participants reported eating breakfast more often and eating more fruits and consuming less fruit juice, sugar-sweetened beverages and unhealthy snacks (small to medium effect sizes). Changes regarding sugar-sweetened beverages and consumption of unhealthy snacks were maintained in the longer term.

\section{Quality of life and BMI}

The participants reported a significantly better quality of life for the dimensions of selfcare, pain and anxiety (medium effect size) at post-test. The pain and anxiety dimensions remained improved at T2. This led to better total quality of life scores at T1 and T2. Health in general and perceived health on the day they completed the questionnaire, which are also quality of life indicators, had increased at T2 and health in general had also increased at T1 (medium effect sizes).

The participants lost an average of $2.3 \mathrm{~kg}$ immediately after completing the CooL intervention, which corresponds to $0.8 \mathrm{BMI}$ points. At $\mathrm{T} 2$, the average weight loss was still $1.8 \mathrm{~kg}$, compared to the pre-test. In total, $63.2 \%$ of the participants had lost weight at T1; $25.0 \%$ of the participants had lost more than $5 \%$ body weight; and $44.3 \%$ of the participants had lost more than $2 \%$ body weight (data not shown). At T2, 61.8\% of the participants had lost weight compared to baseline, while $21.6 \%$ had lost more than $5 \%$ weight and $39.6 \%$ had lost more than $2 \%$ of their original weight.

\section{Changes among the children participants}

Figure 2 shows all the healthy changes among the parents and children regarding parenting, motivation, behaviours, quality of life and BMIz. All aggregated effect sizes clustered around 0.10 at T2 (small effect sizes), except for the behaviours (with an average effect size of 0.20).

\section{General parenting and parenting practices}

At T1, parents experienced fewer problems with their child's behaviour compared to the pre-test, with a small effect size ( -0.5 on a 7-point Likert scale; Table 3$)$. In addition, their self-efficacy as regards managing these problems had increased at $\mathrm{T1}$, with a large effect size. After the programme, parents showed a healthier role model behaviour regarding healthy eating and being physically active in the presence of their child (medium to large effect sizes). This role modelling for healthy eating was maintained at T2. The children's food environment had also improved at T1 and T2, with parents providing more healthy food for their children (medium to large effect sizes). In addition, their so-called 'covert control' practices had also changed at T1 and T2, with medium effect sizes: parents more often tried to influence their children's intake of unhealthy products in a way that was not directly visible to the child, for example by reducing the availability or visibility of unhealthy food products at home. 


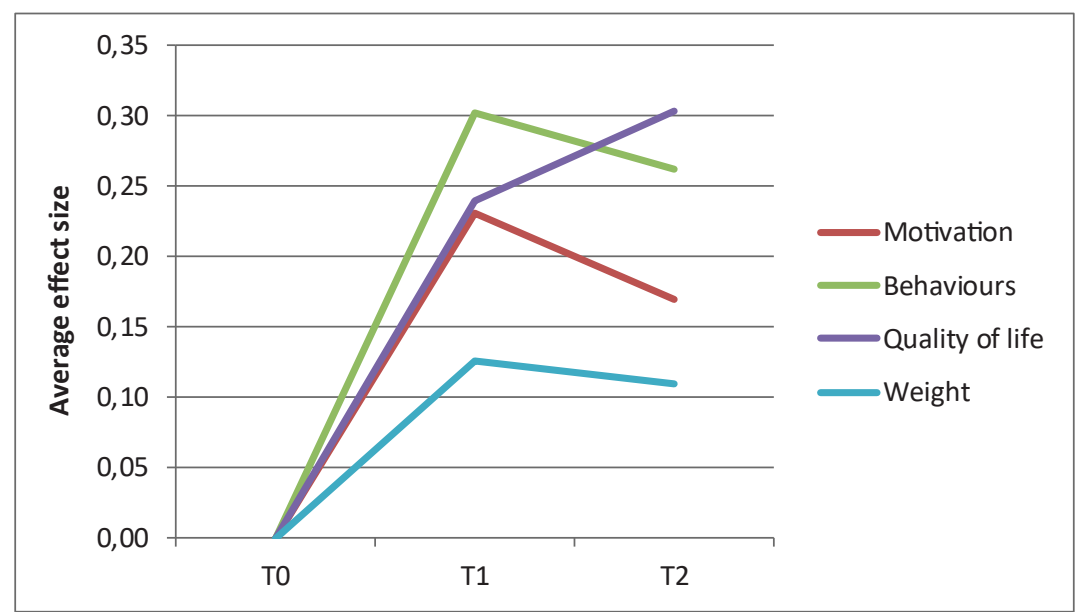

Figure 1: Overall changes among the adults regarding motivation, behaviours, quality of life and weight

\section{Motivation and behaviour-specific cognitions}

The children appeared not to be affected by the intervention as regards the motivational sub-scales for physical activity, since no statistically significant changes from baseline were detected. However, medium effect sizes were found regarding their intrinsic motivation at T1 and their introject regulation at T2. Children's self-reported attitude towards fruit showed a significant negative change after the intervention compared with the pre-test (medium effect size). No significant changes were observed regarding enjoyment of physical activity, habit strength for physical activity and healthy eating, or regarding self-efficacy. Medium effect sizes were found at T1 or T2 for habit strength for playing outside and eating and self-efficacy regarding playing outside.

\section{Energy balance-related behaviours}

Immediately after completing the intervention, as well as in the longer term, the children were spending significantly less time watching television compared with the pretest ( -2.1 and -4.5 hours per week, respectively) as reported by their parents, with small to large effect sizes. A large effect size was found regarding the sports behaviour reported by the children at $\mathrm{T} 2$. The parents reported that their child was eating more vegetables and was drinking water more often and was drinking sugar-sweetened beverages and fruit juices less often, at T1 or T2, with small to large effect sizes. Medium to large effect sizes were found at T1 for eating fruit and unhealthy snacks and drinking diet beverages. Regarding the other physical activity and dietary behaviours, small and non-significant changes were found. At the pre-test, $23 \%$ of the children suffered from sleeping problems (data not shown). The number of sleeping problems had not significantly decreased after the intervention.

\section{Quality of life and BMIz}

At post-test, the physical comfort dimension and the overall quality of life score had improved significantly compared to the pre-test (small effect sizes). Parents reported that their child was able to move better and with greater ease. We found medium effect siz- 
62 Chapter 4

Table 2: Average changes among adults regarding psychological needs, motivation for physical activity and healthy diet, barriers, physical activity, dietary behaviour, quality of life and BMI, at T1 and T2 compared to T0, analysed using linear mixed models

\begin{tabular}{|c|c|c|c|c|c|}
\hline \multirow[b]{2}{*}{ Outcome variable } & \multirow{2}{*}{$\begin{array}{l}\text { Pre-test (T0) } \\
\text { Mean (SD) }\end{array}$} & \multicolumn{2}{|c|}{ Change T1-T0 } & \multicolumn{2}{|c|}{ Change T2-T0 } \\
\hline & & $\mathrm{B}(\mathrm{Cl})$ & Effect size & $\mathrm{B}(\mathrm{Cl})$ & Effect size \\
\hline \multicolumn{6}{|l|}{ Psychological needs ${ }^{1}$} \\
\hline Autonomy & $4.3(0.7)$ & $0.2(0.0,0.3)^{* *}$ & 0.31 & $0.2(0.1,0.3)^{* *}$ & 0.40 \\
\hline Competence & $3.3(0.9)$ & $0.2(0.0,0.4)$ & 0.27 & $0.2(-0.1,0.4)$ & 0.29 \\
\hline Relatedness & $3.4(1.0)$ & $-0.1(-0.3,0.1)$ & 0.04 & $-0.1(-0.4,0.1)$ & -0.04 \\
\hline \multicolumn{6}{|l|}{ Motivation for physical activity ${ }^{2}$} \\
\hline Amotivation & $0.6(0.7)$ & $0.0(-0.1,0.2)$ & -0.01 & $0.0(-0.2,0.2)$ & -0.01 \\
\hline External regulation & $0.9(1.1)$ & $-0.3(-0.4,-0.1)^{* *}$ & -0.24 & $-0.3(-0.5,-0.2)^{* * *}$ & -0.28 \\
\hline Introjected regulation & $1.4(1.0)$ & $0.0(-0.2,0.2)$ & 0.05 & $-0.2(-0.4,0.1)$ & -0.14 \\
\hline Controlled motivation ${ }^{3}$ & $5.0(3.9)$ & $-0.4(-1.2,0.3)$ & -0.14 & $-0.8(-1.6,0.0)^{*}$ & -0.21 \\
\hline Identified regulation & $2.8(0.9)$ & $0.1(-0.1,0.3)$ & 0.16 & $0.0(-0.2,0.1)$ & 0.00 \\
\hline Integrated regulation & $2.2(1.1)$ & $0.3(0.1,0.5)^{* * *}$ & 0.30 & $0.2(0.0,0.4)$ & 0.14 \\
\hline Intrinsic motivation & $2.5(1.2)$ & $0.3(0.1,0.5)^{* * *}$ & 0.36 & $0.2(0.0,0.4)^{*}$ & 0.16 \\
\hline Autonomous motivation ${ }^{4}$ & $14.7(6.3)$ & $1.9(0.9,2.9)^{* * *}$ & 0.33 & $1.0(0.0,1.9)$ & 0.14 \\
\hline \multicolumn{6}{|l|}{ Motivation for healthy diet ${ }^{2}$} \\
\hline Amotivation & $0.6(0.8)$ & $0.0(-0.2,0.1)$ & -0.06 & $0.0(-0.1,0.2)$ & 0.04 \\
\hline External regulation & $2.0(1.0)$ & $-0.2(-0.4,-0.1)^{*}$ & -0.16 & $-0.2(-0.4,0.0)$ & -0.15 \\
\hline Introjected regulation & $2.1(1.0)$ & $-0.2(-0.4,0.0)^{*}$ & -0.14 & $-0.2(-0.4,0.0)^{*}$ & -0.19 \\
\hline Controlled motivation ${ }^{3}$ & $8.0(3.9)$ & $-0.9(-1.5,-0.2)^{*}$ & -0.21 & $-0.5(-1.3,0.3)$ & -0.10 \\
\hline Identified regulation & $3.6(0.6)$ & $0.0(-0.2,0.1)$ & -0.01 & $-0.1(-0.2,0.1)$ & -0.09 \\
\hline Integrated regulation & $2.6(1.0)$ & $0.4(0.2,0.6)^{* * *}$ & 0.52 & $0.3(0.1,0.5)^{* *}$ & 0.34 \\
\hline Intrinsic motivation & $2.9(0.9)$ & $0.2(0.1,0.4)^{* * *}$ & 0.38 & $0.0(-0.1,0.2)$ & 0.10 \\
\hline Autonomous motivation ${ }^{4}$ & $17.5(4.6)$ & $1.6(0.9,2.2)^{* * *}$ & 0.46 & $0.7(-0.2,1.5)$ & 0.19 \\
\hline \multicolumn{6}{|l|}{ Barriers $^{1}$} \\
\hline Physical activity & $2.5(0.8)$ & $-0.3(-0.5,-0.2)^{* * *}$ & -0.52 & $-0.3(-0.4,-0.2)^{* * *}$ & -0.44 \\
\hline Eating a healthy diet & $2.1(0.8)$ & $-0.3(-0.5,-0.2)^{* * *}$ & -0.51 & $-0.3(-0.5,-0.1)^{* * *}$ & -0.44 \\
\hline \multicolumn{6}{|l|}{ Physical activity } \\
\hline Sedentary behaviour ${ }^{5}$ & $437.1(198.8)$ & $\begin{array}{l}-123.2(-162.2 \\
-84.2)^{* * *}\end{array}$ & -0.69 & $\begin{array}{l}-102.9(-151.6 \\
-54.2)^{* * *}\end{array}$ & -0.52 \\
\hline Walking ${ }^{6}$ & $284.3(327.9)$ & $-35.3(-101.4,30.7)$ & -0.15 & $-12.1(-83.2,59.0)$ & -0.06 \\
\hline Moderate-intensity activities ${ }^{6}$ & $229.6(326.5)$ & $72.2(-23.3,167.7)$ & 0.25 & $106.8(16.5,197.0)^{*}$ & 0.32 \\
\hline Vigorous-intensity activities ${ }^{6}$ & $96.2(167.7)$ & $78.2(26.3,130.0)^{* *}$ & 0.41 & $87.5(25.4,149.5)^{* *}$ & 0.50 \\
\hline Total & $593.8(662.6)$ & $131.6(-22.6,285.8)$ & 0.18 & $202.0(37.9,366.1)^{*}$ & 0.28 \\
\hline \multicolumn{6}{|l|}{ Dietary behaviour } \\
\hline Breakfast $^{7}$ & $6.3(1.8)$ & $0.5(0.2,0.8)^{* *}$ & 0.24 & $0.2(-0.1,0.5)$ & 0.11 \\
\hline Fruits ${ }^{8}$ & $9.4(7.6)$ & $1.8(0.3,3.2)^{*}$ & 0.26 & $0.8(-0.6,2.1)$ & 0.21 \\
\hline Vegetables $^{9}$ & $26.8(12.4)$ & $2.4(0.0,4.7)$ & 0.21 & $3.0(-0.9,6.9)$ & 0.18 \\
\hline Fruit juices ${ }^{7}$ & $2.1(2.3)$ & $-0.7(-1.1,-0.3)^{* * *}$ & -0.33 & $-0.5(-1.0,0.0)$ & -0.28 \\
\hline
\end{tabular}


Table 2: continued

\begin{tabular}{|c|c|c|c|c|c|}
\hline \multirow[b]{2}{*}{ Outcome variable } & \multirow{2}{*}{$\begin{array}{l}\text { Pre-test (T0) } \\
\text { Mean (SD) }\end{array}$} & \multicolumn{2}{|c|}{ Change T1-T0 } & \multicolumn{2}{|c|}{ Change T2-T0 } \\
\hline & & $\mathrm{B}(\mathrm{Cl})$ & Effect size & B (CI) & Effect size \\
\hline Sugar-sweetened beverages ${ }^{7}$ & $2.7(2.9)$ & $-1.0(-1.5,-0.6)^{* * *}$ & -0.44 & $-1.0(-1.5,-0.5)^{* * *}$ & -0.40 \\
\hline Unhealthy snacks ${ }^{7}$ & $8.7(6.3)$ & $-2.5(-3.6,-1.5)^{* * *}$ & -0.33 & $-1.5(-2.9,-0.1)^{*}$ & -0.17 \\
\hline \multicolumn{6}{|l|}{ Quality of life } \\
\hline Mobility ${ }^{10}$ & $1.5(0.5)$ & $-0.1(-0.2,0.0)$ & -0.16 & $0.0(-0.1,0.0)$ & -0.24 \\
\hline Self-care ${ }^{10}$ & $1.1(0.3)$ & $-0.1(-0.1,0.0)^{*}$ & -0.20 & $0.0(-0.1,0.1)$ & -0.07 \\
\hline Usual activities ${ }^{10}$ & $1.4(0.5)$ & $0.0(-0.1,0.0)$ & -0.11 & $-0.1(-0.2,0.1)$ & -0.19 \\
\hline Pain/discomfort ${ }^{10}$ & $1.8(0.6)$ & $-0.1(-0.2,0.0)^{*}$ & -0.19 & $-0.1(-0.2,0.0)^{*}$ & -0.29 \\
\hline Anxiety/depression ${ }^{10}$ & $1.5(0.6)$ & $-0.2(-0.3,-0.1)^{* * *}$ & -0.37 & $-0.2(-0.3,-0.1)^{* *}$ & -0.35 \\
\hline Total $^{11}$ & $0.7(0.3)$ & $0.1(0.0,0.1)^{* *}$ & 0.24 & $0.1(0.0,0.1)^{*}$ & 0.32 \\
\hline In general ${ }^{12}$ & $6.3(1.4)$ & $0.5(0.2,0.7)^{* * *}$ & 0.42 & $0.7(0.4,0.9)^{* * *}$ & 0.57 \\
\hline At that moment ${ }^{12}$ & $6.5(1.3)$ & $0.3(0.0,0.5)$ & 0.22 & $0.5(0.2,0.8)^{* *}$ & 0.42 \\
\hline \multicolumn{6}{|l|}{ Weight and BMI } \\
\hline Weight ${ }^{13}$ & $105.4(19.7)$ & $-2.3(-3.3,-1.3)^{* * *}$ & -0.13 & $-1.8(-2.8,-0.8)^{* * *}$ & -0.11 \\
\hline $\mathrm{BMI}^{14}$ & $36.2(5.9)$ & $-0.8(-1.1,-0.4)^{* * *}$ & -0.13 & $-0.6(-0.9,-0.3)^{* * *}$ & -0.14 \\
\hline
\end{tabular}

Notes: Results of linear mixed model analyses (unstructured covariance type); analyses were controlled for background characteristics if they were significantly related to missingness: age, gender, living situation, occupational status, highest completed education and $\mathrm{BMI} ; \mathrm{SD}=$ standard deviation, $\mathrm{B}$ is the corrected estimated mean difference in change scores from baseline, $\mathrm{Cl}=$ confidence interval, significantly different $\left(^{*}=p<0.050 ;{ }^{* *}=p<0.010 ; * * *=p<0.001\right) ;{ }^{1}$ five-point scale $(1=$ totally disagree; $5=$ totally agree $),{ }^{2}$ five-point scale $(0=$ totally disagree; $4=$ totally agree $),{ }^{3}$ sum score $(0=$ uncontrolled motivation; 24 = controlled motivation), ${ }^{4}$ sum score ( 0 = no autonomous motivation; 24 = autonomous motivation), ${ }^{5}$ outcome measured in minutes per day, ${ }^{6}$ minutes per week, ${ }^{7}$ frequency per week, ${ }^{8}$ pieces per week, ${ }^{9}$ serving spoons per week, ${ }^{10}$ three-point scale $\left(1=\right.$ no disability; 3 = greatly disabled), ${ }^{11}$ correction factor $\left(-0.33=\right.$ completely unhealthy; 1 = completely healthy), ${ }^{12}$ grade (1-10); ${ }^{13}$ in $\mathrm{kg} ;{ }^{14}$ in $\mathrm{kg} / \mathrm{m}^{2}$.

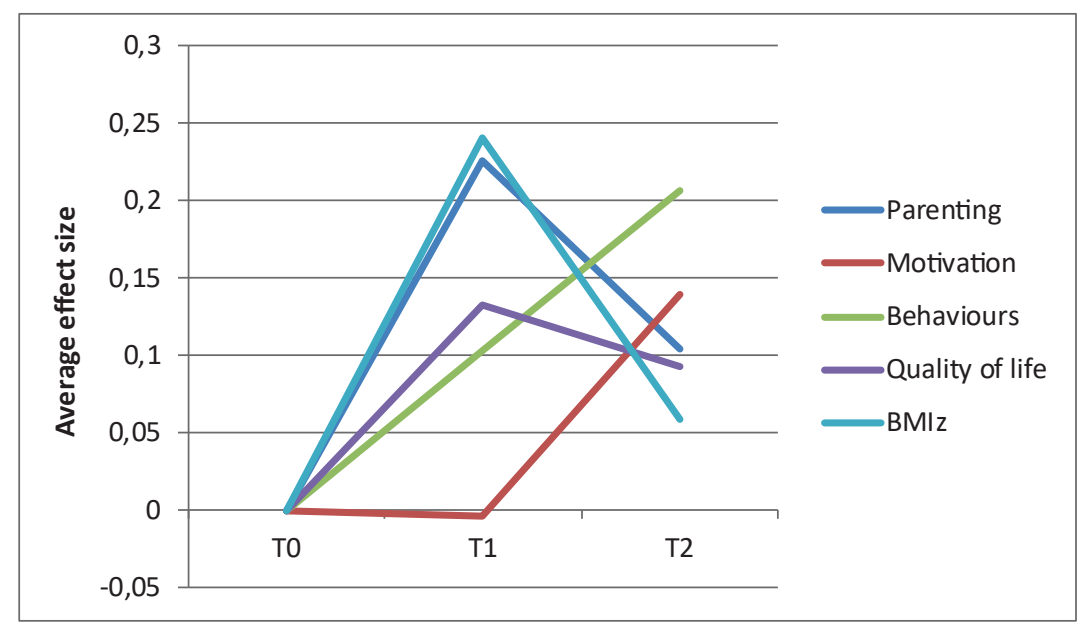

Figure 2: Overall changes among the parents and children regarding parenting, motivation, behaviours, quality of life and BMIz. 
64 Chapter 4

Table 3: Average change reported by parents and children regarding parenting, motivation for physical activity and healthy diet, behaviour-specific cognitions, physical activity, dietary behaviour, sleeping behaviour, quality of life and BMIz, at T1 and T2, analysed with linear mixed models

\begin{tabular}{|c|c|c|c|c|c|}
\hline \multirow[b]{2}{*}{ Outcome variable } & \multirow{2}{*}{$\begin{array}{l}\text { Pre-test (T0) } \\
\text { Mean (SD) }\end{array}$} & \multicolumn{2}{|c|}{ Change T1-T0 } & \multicolumn{2}{|c|}{ Change T2-T0 } \\
\hline & & $\mathrm{B}(\mathrm{Cl})$ & Effect size & $\mathrm{B}(\mathrm{Cl})$ & Effect size \\
\hline \multicolumn{6}{|l|}{ General parenting } \\
\hline Monitoring ${ }^{1 a}$ & $3.4(1.0)$ & $0.0(-0.6,0.7)$ & 0.12 & $-0.1(-0.7,0.5)$ & -0.01 \\
\hline Consistent discipline ${ }^{1 a}$ & $2.8(1.3)$ & $0.2(-0.3,0.8)$ & 0.19 & $0.3(-0.4,1.1)$ & 0.28 \\
\hline Autonomy support ${ }^{1 a}$ & $4.1(1.0)$ & $-0.1(-0.6,0.3)$ & 0.01 & $0.2(-0.3,0.7)$ & 0.18 \\
\hline Problem scale ${ }^{2}$ & $2.7(1.1)$ & $-0.5(-0.8,-0.1)^{*}$ & -0.24 & $-0.3(-0.8,0.2)$ & -0.23 \\
\hline Confidence scale $^{3 b}$ & $6.6(2.3)$ & $1.3(0.3,2.4)^{* *}$ & 0.74 & $0.4(-0.9,1.8)$ & 0.22 \\
\hline \multicolumn{6}{|l|}{ Parenting practices } \\
\hline $\begin{array}{l}\text { Stimulation to be active and to eat a } \\
\text { healthy diet }{ }^{1}\end{array}$ & $4.4(0.5)$ & $0.1(-0.1,0.3)$ & 0.15 & $0.0(-0.2,0.3)$ & 0.13 \\
\hline $\begin{array}{l}\text { Parental role modelling - healthy } \\
\text { eating }{ }^{1}\end{array}$ & $4.0(0.6)$ & $0.3(0.1,0.6)^{* *}$ & 0.51 & $0.3(0.0,0.5)^{*}$ & 0.59 \\
\hline $\begin{array}{l}\text { Parental role modelling - physical } \\
\text { activity }{ }^{4 a}\end{array}$ & $3.1(0.6)$ & $0.4(0.2,0.6)^{* * *}$ & 0.66 & $0.1(-0.1,0.3)$ & 0.19 \\
\hline Food environment ${ }^{1 a}$ & $3.5(1.0)$ & $0.4(0.0,0.8)^{*}$ & 0.35 & $0.5(0.1,1.0)^{*}$ & 0.65 \\
\hline Parental policies ${ }^{4 a}$ & $3.9(0.8)$ & $0.1(-0.1,0.4)$ & 0.35 & $-0.1(-0.4,0.2)$ & -0.06 \\
\hline Emotional feeding ${ }^{4}$ & $1.3(0.5)$ & $0.0(-0.1,0.2)$ & -0.03 & $0.2(-0.1,0.5)$ & 0.32 \\
\hline Instrumental feeding ${ }^{4}$ & $1.5(0.6)$ & $0.0(-0.1,0.2)$ & -0.22 & $0.0(-0.2,0.3)$ & 0.15 \\
\hline Monitoring physical activity ${ }^{4}$ & $3.7(0.7)$ & $0.2(0.0,0.3)$ & 0.30 & $-0.2(-0.5,0.2)$ & -0.25 \\
\hline Covert control ${ }^{4}$ & $2.9(0.9)$ & $0.3(0.0,0.6)^{*}$ & 0.35 & $0.3(0.0,0.7)^{*}$ & 0.48 \\
\hline Child control ${ }^{4}$ & $2.4(0.7)$ & $0.2(-0.1,0.5)$ & 0.22 & $0.1(-0.2,0.3)$ & 0.07 \\
\hline \multicolumn{6}{|l|}{ Motivation for physical activity ${ }^{1}$} \\
\hline Amotivation $^{a}$ & $0.8(1.2)$ & $-0.1(-0.5,0.3)$ & 0.00 & $-0.3(-0.8,0.2)$ & -0.12 \\
\hline External regulation ${ }^{b}$ & $0.8(0.7)$ & $-0.1(-0.8,0.6)$ & -0.21 & $0.0(-0.5,0.4)$ & -0.09 \\
\hline Introjected regulation & $1.0(0.6)$ & $0.2(-0.1,0.4)$ & 0.13 & $-0.2(-0.6,0.1)$ & -0.38 \\
\hline Controlled motivation ${ }^{5}$ & $5.2(5.0)$ & $0.5(-1.4,2.5)$ & -0.11 & $-0.4(-3.4,2.7)$ & -0.19 \\
\hline Identified regulation & $2.3(1.0)$ & $-0.2(-0.9,0.4)$ & -0.03 & $0.1(-0.4,0.5)$ & 0.27 \\
\hline Intrinsic motivation & $2.7(1.1)$ & $0.1(-0.3,0.6)$ & 0.48 & $0.0(-0.6,0.6)$ & 0.21 \\
\hline Autonomous motivation ${ }^{6}$ & $12.7(5.2)$ & $-1.1(-2.7,0.4)$ & 0.30 & $-0.4(-3.2,2.4)$ & 0.24 \\
\hline \multicolumn{6}{|l|}{ Behaviour-specific cognitions } \\
\hline Physical activity enjoyment ${ }^{1}$ & $4.0(0.8)$ & $0.0(-0.3,0.3)$ & -0.18 & $-0.1(-0.3,0.1)$ & -0.14 \\
\hline Habit strength - sports $^{1}$ & $3.5(1.0)$ & $-0.1(-0.5,0.4)$ & -0.29 & $-0.1(-0.4,0.3)$ & -0.18 \\
\hline Habit strength - playing outside ${ }^{1}$ & $3.3(1.1)$ & $0.2(-0.3,0.7)$ & 0.38 & $-0.1(-0.7,0.5)$ & 0.23 \\
\hline Habit strength - eating fruits ${ }^{1}$ & $3.2(1.1)$ & $0.1(-0.2,0.4)$ & 0.42 & $0.4(-0.2,1.0)$ & 0.40 \\
\hline Self-efficacy - playing outside ${ }^{7}$ & $2.9(1.0)$ & $-0.1(-0.6,0.3)$ & -0.23 & $0.1(-0.5,0.6)$ & 0.41 \\
\hline Self-efficacy - eating fruits ${ }^{7}$ & $3.2(0.6)$ & $-0.1(-0.4,0.2)$ & -0.26 & $0.0(-0.4,0.3)$ & 0.09 \\
\hline Attitude - eating fruits ${ }^{1}$ & $4.1(0.6)$ & $-0.3(-0.5,0.0)^{*}$ & -0.42 & $-0.2(-0.4,0.1)$ & -0.20 \\
\hline \multicolumn{6}{|l|}{ Physical activity ${ }^{8}$} \\
\hline Watching television & $9.8(5.9)$ & $-2.1(-4.0,-0.2)^{*}$ & -0.23 & $-4.5(-6.6,-2.4)^{* * *}$ & -0.91 \\
\hline
\end{tabular}


Table 3: continued

\begin{tabular}{|c|c|c|c|c|c|}
\hline \multirow[b]{2}{*}{ Outcome variable } & \multirow{2}{*}{$\begin{array}{l}\text { Pre-test (TO) } \\
\text { Mean (SD) }\end{array}$} & \multicolumn{2}{|c|}{ Change T1-T0 } & \multicolumn{2}{|c|}{ Change T2-T0 } \\
\hline & & $\mathrm{B}(\mathrm{Cl})$ & Effect size & $\mathrm{B}(\mathrm{Cl})$ & Effect size \\
\hline Using a computer & $9.2(7.2)$ & $0.5(-2.0,3.0)$ & 0.10 & $1.7(-0.9,4.3)$ & 0.28 \\
\hline Playing outside & $7.4(6.7)$ & $-1.1(-3.4,1.2)$ & -0.10 & $-1.8(-4.8,1.1)$ & -0.29 \\
\hline Engaging in sports & $3.1(3.6)$ & $0.4(-0.8,1.6)$ & 0.00 & $-0.4(-1.4,0.7)$ & -0.20 \\
\hline Walking or biking to school & $3.0(3.9)$ & $0.6(-0.3,1.5)$ & -0.08 & $0.2(-1.4,1.9)$ & 0.09 \\
\hline Walking or biking during leisure time & $4.6(5.7)$ & $-0.2(-2.2,1.9)$ & -0.10 & $-1.4(-3.4,0.5)$ & -0.27 \\
\hline Sports behaviour ${ }^{9 b}$ & $3.0(1.5)$ & $-0.9(-2.2,0.5)$ & -0.22 & $1.5(-0.3,3.2)$ & 0.66 \\
\hline \multicolumn{6}{|l|}{ Dietary behaviour } \\
\hline Breakfast $^{9}$ & $6.0(1.8)$ & $0.5(-0.1,1.1)$ & 0.24 & $0.0(-0.4,0.4)$ & 0.08 \\
\hline Fruits ${ }^{10}$ & $9.3(6.3)$ & $2.8(-2.3,8.0)$ & 0.58 & $0.6(-1.5,2.7)$ & 0.03 \\
\hline Vegetables ${ }^{11 \mathrm{~b}}$ & 14.5 (9.9) & $6.6(0.8,12.3)^{*}$ & 0.68 & $5.9(-1.1,13.0)$ & 0.92 \\
\hline Water $^{12}$ & $16.2(16.8)$ & $12.7(3.2,22.1)^{*}$ & 0.61 & $8.3(1.4,15.3)^{*}$ & 0.27 \\
\hline Sugar-sweetened beverages ${ }^{12}$ & $8.3(11.2)$ & $-3.9(-11.0,3.3)$ & -0.23 & $-5.6(-10.5,-0.8)^{*}$ & -0.55 \\
\hline Diet beverages ${ }^{12}$ & $2.7(5.3)$ & $2.7(2.7,8.0)$ & 0.61 & $-0.5(-2.6,1.5)$ & -0.09 \\
\hline Fruit beverages ${ }^{12}$ & $1.9(2.8)$ & $-0.4(-3.0,2.3)$ & -0.10 & $-1.2(-2.4,0.0)^{*}$ & -0.47 \\
\hline Fruit juices ${ }^{12}$ & $1.6(2.4)$ & $-1.1(-2.0,-0.2)^{*}$ & -0.40 & $-1.0(-2.0,0.0)$ & -0.41 \\
\hline Energy drinks ${ }^{12 b}$ & $0.1(0.5)$ & $0.1(-0.2,0.4)$ & 0.19 & $0.0(-0.3,0.3)$ & -0.05 \\
\hline Unhealthy snacks $^{9}$ & $8.3(4.2)$ & $-0.9(-2.6,0.7)$ & -0.33 & $-0.8(-2.1,0.5)$ & -0.17 \\
\hline \multicolumn{6}{|l|}{ Sleeping behaviour } \\
\hline Sleeping hours ${ }^{8}$ & $48.5(7.8)$ & $1.2(-2.4,4.8)$ & 0.16 & $0.5(-3.3,4.3)$ & 0.12 \\
\hline Sleeping problems ${ }^{13}$ & $0.3(0.7)$ & $0.0(-0.3,0.4)$ & 0.22 & $-0.1(-0.4,0.2)$ & -0.13 \\
\hline \multicolumn{6}{|l|}{ Quality of life } \\
\hline Physical comfort ${ }^{14}$ & $25.2(5.3)$ & $1.9(0.2,3.5)^{*}$ & 0.25 & $1.4(-0.6,3.4)$ & 0.35 \\
\hline Body esteem ${ }^{14}$ & $35.4(8.2)$ & $1.8(-0.7,4.2)$ & 0.06 & $0.0(-3.0,3.1)$ & 0.01 \\
\hline Social life ${ }^{14}$ & $26.0(4.4)$ & $0.4(-0.8,1.6)$ & 0.07 & $0.8(-0.3,1.8)$ & 0.14 \\
\hline \multirow[t]{2}{*}{ Family relations ${ }^{14}$} & $28.3(2.4)$ & $0.8(-0.3,1.9)$ & 0.35 & $0.7(-0.5,1.8)$ & 0.18 \\
\hline & $115.2(16.5)$ & $4.4(0.8,8.1)^{*}$ & 0.16 & $2.2(-3.4,7.7)$ & 0.15 \\
\hline Health of parent in general ${ }^{3}$ & $7.6(1.0)$ & $-0.1(-0.5,0.3)$ & -0.13 & $-0.2(-0.6,0.3)$ & -0.05 \\
\hline Health of parent at that moment ${ }^{3}$ & $7.5(1.1)$ & $0.0(-0.6,0.7)$ & -0.07 & $-0.2(-0.7,0.4)$ & -0.10 \\
\hline Health of child in general ${ }^{3}$ & $7.4(1.5)$ & $0.3(-0.1,0.8)$ & 0.22 & $-0.1(-0.6,0.4)$ & 0.08 \\
\hline Health of child at that moment ${ }^{3}$ & $7.4(1.6)$ & $0.5(0.0,1.1)$ & 0.31 & $-0.1(-0.6,0.4)$ & 0.13 \\
\hline \multicolumn{6}{|l|}{ BMIz } \\
\hline $\mathrm{BMIz}{ }^{16}$ & $2.4(0.4)$ & $-0.1(-0.2,0.0)$ & -0.24 & $0.0(-0.1,0.1)$ & -0.06 \\
\hline
\end{tabular}

Notes: Results of linear mixed model analyses (unstructured covariance type); analyses were controlled for background characteristics if they were significantly related to missingness: age, gender, living situation, occupational status, highest completed education and $\mathrm{BMIz} ; \mathrm{SD}=$ standard deviation, $\mathrm{B}$ is the corrected estimated mean difference in change scores from baseline,

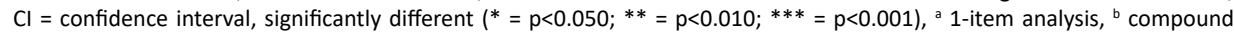
symmetry analysis; ${ }^{1}$ five-point scale $\left(1=\right.$ totally disagree; $5=$ totally agree), ${ }^{2}$ seven-point scale $(1=$ no problem at all; $7=$ very much a problem), ${ }^{3}$ grade $(1-10),{ }^{4}$ five-point scale $(1=$ never; $5=$ always $),{ }^{5}$ sum score $(0=$ uncontrolled motivation; $24=$ controlled motivation), ${ }^{6}$ sum score $(0=$ no autonomous motivation; $20=$ autonomous motivation $),{ }^{7}$ five-point scale $(1=$ very hard; $5=$ not hard at all), ${ }^{8}$ hours per week, ${ }^{9}$ frequency per week, ${ }^{10}$ pieces per week, ${ }^{11}$ serving spoons per week, ${ }^{12}$ cups per week, ${ }^{13}$ five-point scale $(0=$ no problems; $1=1$ problem; $4=4$ problems $),{ }^{14}$ five-point scale $(1=$ always true; $5=$ never true $)$, ${ }^{15}$ scale $(1$ = worse; 135 = best $),{ }^{16}$ standardized BMI. 
es for the family relations dimension at $\mathrm{T} 1$ and the physical comfort dimension at $\mathrm{T} 2$. On average, children did not differ significantly in terms of their BMIz after the intervention.

\section{DISCUSSION}

In this study we monitored the motivational, behavioural and weight changes among adults and children with obesity or overweight participating in the CooL intervention over a period of 1.5 years. Among the adult participants, we found significant improvements regarding perceived autonomy, motivational regulation for physical activity and healthy diet, perceived barriers to engaging in physical activity and eating a healthy diet, physical activity, healthy dietary behaviours and quality of life. Adults lost an average of $2.3 \mathrm{~kg}$ of body weight, which was largely maintained approximately one year later. These findings are relatively favourable compared to what was found in previous studies on implemented 'real-world' CLIs [38, 51, 137-139], in which the body weight change varied between losses of $0.6 \mathrm{~kg}$ [41] and $3.0 \mathrm{~kg}$ over a study period of one year or longer [141, 142].

The children in our study, however, showed less significant changes and smaller effect sizes. We found significant improvements regarding the time spent watching television, dietary behaviours, physical comfort and overall quality of life score. In contrast, children developed a significantly more negative attitude towards fruit consumption. They did not significantly change their BMIz score. In general, there were no significant changes regarding the children's physical activity, motivation, habit formation or weight. However, medium to large effects sizes were found for some motivational, behavioural and quality of life outcomes. Their parents, however, perceived significantly less problems with their child's behaviour, they increased their self-efficacy as regards addressing these issues and they improved their role modelling regarding healthy eating and physical activity, food environment and covert control.

One of the main aspects of the CooL approach is an autonomy-supportive coaching style, which has been shown to predict a shift towards more autonomous types of motivation among participants [83]. Motivational regulation for physical activity and healthy diet is a predictor of changing and maintaining healthier behaviour [10, 143]. Among our adult population we did indeed observe some changes towards more autonomous types of motivation. The adults showed more autonomous types of motivation at T1. Although this was not fully maintained at $\mathrm{T} 2$, the moderate and vigorous physical activity scores had even increased at $\mathrm{T} 2$. This result may imply that improved quality of motivation functions as a mediator to sustained behavioural change. An improved quality of motivation may be needed to induce behavioural changes, after which other habitual or routine processes play a role in maintaining these changes [144-146].

CLIs are typically carried out by multiple professionals, with a strong basis in primary care (e.g. general practices). Ours was the first study that investigated the effects of a lifestyle coaching intervention within The Netherlands in which a lifestyle coach was positioned as the central health professional, outside the traditional primary care chain. 
One of the outcomes from earlier studies [72] was that the participating professionals were undereducated and lacked resources (including sufficient time) to optimally counsel participants regarding their lifestyle. The post-graduated lifestyle coaches in our study showed that they were able to sustainably change the lifestyle of their participants using a step-by-step approach focusing on an autonomy-supportive coaching style that combines client-tailored individual sessions with more general group sessions. Note that lifestyle coaches in real-life settings need to be highly adaptive to the health care context, client profiles and public health systems.

A clear difference between the adults and children was observed regarding maintained changes. Firstly, in comparison to adults, it proved to be harder to include children (and their parents) in the intervention. Recruitment issues have been encountered previously in childhood obesityinterventions [147]. Reasons for this include denial of the problem by parents and resistance of parents towards discussing weight issues, as well as professionals who feel unable (through lack of skills or self-efficacy) to motivate parents to participate in obesity interventions and inhibiting societal norms regarding weight and participation in child overweight programmes. The adult participant recruitment was also insufficient, since the study sample was smaller than expected. Apart from recruitment issues, it may also be more difficult to achieve behavioural change in children, since they often have complex family situations and rooted routines. Another reason can be the complexity of the child's obesity problem. When a child or its family are facing additional problems, it might not be the best time to start a lifestyle intervention. In such situations other, more urgent, problems (e.g. safety and poverty) need to be tackled first, after which other needs (e.g. losing weight) can be discussed [11]. This is a topic for which the coaches should be better trained, or for which coaches could involve with other health professionals more often.

\section{Strengths and limitations}

Strengths of this study include its real-life setting, longitudinal design and the use of validated questionnaires. The combination of multiple outcome variables provides a general overview of cognitive and behavioural changes among the participants. However, in order to shorten the questionnaires, not all the variables were included in every measurement. Another strength was that the group that has been exposed to this CooL intervention was geographically heterogeneous, as it has been implemented in multiple regions within The Netherlands. In addition, the study sample was representative of the Dutch population in terms of educational level and living situation. Note that a relatively large part of the study sample had a low or intermediate educational level. Most comparable studies have had difficulties recruiting this specific target population [139]. In our intervention the participants were recruited via the primary care system (i.e. general practices and YHC professionals), which has been reported to be a good strategy for including participants with a lower or intermediate educational level [139].

Some limitations have to be mentioned too. Including a control group in the design would have been beneficial in terms of making strong statements regarding the effectiveness of the intervention. However, the primary focus of this study was on the imple- 
mentation process (i.e. following the implementation and changes among participants) [123], which reduced the need for a usual care control group. Previous studies on CLIs that did include a control group have shown relative stable patterns of behaviour and weight within the control group [148, 149]. Furthermore, there is also a risk of social desirability in the answers regarding motivation, quality of life and behaviour.

Due to logistical issues, the post-test objective weight data were not measured at the exact same time as the questionnaires were completed. Therefore, we used a wider time frame (several months before and after the T1 or T2) to link these data to the $\mathrm{T} 1$ or T2 data. It is known that overweight or obese individuals underreport their dietary intake [11], height and weight $[150,151]$. In this study, the objectively measured weight and height were combined with the self-reported data to decrease the amount of missing values. This may have led to partially biased data. However, the degree of consistency between objective and self-reported weight data for adults was very high for the cases where we had access to both data sources $(r=0.985)$, which offers some confidence in the internal validity of these data.

\section{CONCLUSIONS}

It can be concluded that the lifestyle coaches in this CooL intervention have been successful in coaching the adult participants who are obese or at high risk of obesity towards sustained behavioural change during the study period. Statistically significant changes and medium to large effect sizes among adults were found concerning cognitive and behavioural lifestyle factors and weight. For children and their parents, mixed results and smaller effect sizes were found. 


\section{APPENDIX A. ADDITIONAL TABLES}

Table A1: Techniques and approaches used in the Coaching on Lifestyle (CooL) intervention

\begin{tabular}{|c|c|}
\hline $\begin{array}{l}\text { Techniques, key terms and approaches used in the } \\
\text { adult programme }\end{array}$ & $\begin{array}{l}\text { Techniques and approaches used in the children } \\
\text { and adolescent programme } \\
\text { All techniques and approaches used for the adult } \\
\text { programme are combined with specific inter- } \\
\text { ventions, because of the specific nature of the } \\
\text { educational relationship between parents and their } \\
\text { children }\end{array}$ \\
\hline Autonomy-supportive coaching & General parenting constructs (control, \\
\hline Celebrating (small) successes & responsiveness and structure) \\
\hline Coaches providing feedback and compliments & Parenting practices (e.g. modelling, rule setting \\
\hline Communication skills of the client & and rewarding) to stimulate desired behaviour \\
\hline Dealing with lapses/relapses and temptations & and to restrict undesired behaviour \\
\hline Estimating the value of (different sources of) & Negotiation skills \\
\hline information & \\
\hline Goal setting & \\
\hline Implementation intentions & \\
\hline Increasing self-efficacy & \\
\hline $\begin{array}{l}\text { Knowledge about healthy lifestyle and behavioural } \\
\text { change }\end{array}$ & \\
\hline Mobilizing social support & \\
\hline Motivational interviewing & \\
\hline Nudging & \\
\hline Ownership & \\
\hline Peer modelling, peer support and peer learning & \\
\hline Positive health and positive psychology & \\
\hline Rewarding & \\
\hline $\begin{array}{l}\text { Self-management, self-monitoring and self-evalu- } \\
\text { ation }\end{array}$ & \\
\hline Stepping out of one's comfort zone & \\
\hline
\end{tabular}


$70 \mid$ Chapter 4

Table A2: Constructs used in the questionnaires for the adult participants, with their corresponding scales

\begin{tabular}{|c|c|c|c|c|}
\hline Construct & Scale & Items & Answering options & $\begin{array}{l}\text { Cronbach's } \\
\text { alpha }\end{array}$ \\
\hline \multicolumn{5}{|l|}{ Personal factors } \\
\hline Demographics & - & 9 & Divers & - \\
\hline Psychological needs & $\begin{array}{l}\text { Psychological Need Satis- } \\
\text { faction in Exercise scale } \\
\text { (PNSE) [90] }\end{array}$ & 18 & Totally disagree / totally agree ${ }^{1}$ & \\
\hline Autonomy & & 6 & & 0.9 \\
\hline Competence & & 6 & & 0.9 \\
\hline Relatedness & & 6 & & 0.9 \\
\hline
\end{tabular}

\begin{tabular}{|c|c|c|c|c|}
\hline \multicolumn{5}{|l|}{ Motivation } \\
\hline $\begin{array}{l}\text { Quality of motivation for } \\
\text { physical activity }\end{array}$ & $\begin{array}{l}\text { Behavioural Regulation } \\
\text { in Exercise Questionnaire } \\
\text { (BREQ-3) }[91,152]\end{array}$ & 23 & Totally disagree / totally agree ${ }^{1}$ & \\
\hline Amotivation (AM) & & 4 & & 0.7 \\
\hline External regulation (EX) & & 4 & & 0.8 \\
\hline Introjected regulation (IJ) & & 3 & & 0.7 \\
\hline Controlled motivation & & & Sum score $=(A M * 3)+(E X * 2)+(I J * 1)$ & \\
\hline Identified regulation (ID) & & 4 & & 0.7 \\
\hline Integrated regulation (IG) & & 4 & & 0.9 \\
\hline Intrinsic motivation (IM) & & 4 & & 0.9 \\
\hline Autonomous motivation & & & Sum score $=(I D * 1)+(I G * 2)+(I M * 3)$ & \\
\hline $\begin{array}{l}\text { Quality of motivation for } \\
\text { healthy diet }\end{array}$ & $\begin{array}{l}\text { Regulation of Eating Be- } \\
\text { haviour Scale (REBS) [92] }\end{array}$ & 24 & Totally disagree / totally agree ${ }^{1}$ & \\
\hline Amotivation & & 4 & & 0.7 \\
\hline External regulation & & 4 & & 0.8 \\
\hline Introjected regulation & & 4 & & 0.7 \\
\hline Controlled motivation & & & Sum score $=(A M * 3)+(E X * 2)+(I J * 1)$ & \\
\hline Identified regulation & & 4 & & 0.7 \\
\hline Integrated regulation & & 4 & & 0.8 \\
\hline Intrinsic motivation & & 4 & & 0.8 \\
\hline Autonomous motivation & & & Sum score $=(I D * 1)+(I G * 2)+(I M * 3)$ & \\
\hline \multicolumn{5}{|l|}{ Behaviour-specific cognitions } \\
\hline \multicolumn{5}{|l|}{ Barriers } \\
\hline For physical activity & & 11 & Totally disagree / totally agree ${ }^{1}$ & 0.8 \\
\hline For eating a healthy diet & & 10 & Totally disagree / totally agree ${ }^{1}$ & 0.8 \\
\hline \multicolumn{5}{|c|}{ Energy balance-related behaviours } \\
\hline Physical activity & $\begin{array}{l}\text { International Physical } \\
\text { Activity Questionnaire } \\
\text { (IPAQ) }\end{array}$ & 7 & Days per week, minutes per day & \\
\hline Sedentary behaviour & & 1 & & \\
\hline Walking & & 2 & & \\
\hline Moderate-intensity activities & & 2 & & \\
\hline
\end{tabular}


Table A2: continued

\begin{tabular}{|c|c|c|c|c|}
\hline Construct & Scale & Items & Answering options & $\begin{array}{l}\text { Cronbach's } \\
\text { alpha }\end{array}$ \\
\hline Vigorous-intensity activities & & 2 & & \\
\hline Dietary behaviours & Shortened Fat List [95] & 20 & Days per week, frequency per day & \\
\hline Breakfast & & 1 & & \\
\hline Fruits & & 2 & & \\
\hline Vegetables & & 4 & & \\
\hline Fruit juices & & 1 & & \\
\hline Sugar-sweetened beverages & & 1 & & \\
\hline Unhealthy snacks & & 7 & & \\
\hline \multicolumn{5}{|l|}{ Quality of life and BMI } \\
\hline Quality of life & EQ-5D-3L [96] & 7 & No disability / strongly disabled ${ }^{2}$ & \\
\hline Mobility & & 1 & & \\
\hline Self-care & & 1 & & \\
\hline Usual activities & & 1 & & \\
\hline Pain/discomfort & & 1 & & \\
\hline Anxiety/depression & & 1 & & \\
\hline Total quality of life & [153] & & $\begin{array}{l}\text { Index = } 1 \text { (complete health) - } 0.071 \\
\text { (if there is a problem on a dimen- } \\
\text { sion) - deduction per problem on a } \\
\text { dimension }\end{array}$ & \\
\hline In general & & 1 & & \\
\hline At that moment & & 1 & & \\
\hline$B M I$ & [7] & 2 & Centimetres, kilograms & \\
\hline
\end{tabular}

Notes: ${ }^{1}$ Five-point scale, ${ }^{2}$ three-point scale. 
$72 \mid$ Chapter 4

Table A3: Constructs used in the questionnaires for the children and their parents, with their corresponding scales

\begin{tabular}{|c|c|c|c|c|c|}
\hline Construct & Scale & Items & Answering options & $\begin{array}{l}\text { Cronbach's } \\
\text { alpha }\end{array}$ & Group \\
\hline \multicolumn{6}{|l|}{ Personal factors } \\
\hline Demographics & - & 16 & Divers & - & Parent \\
\hline \multicolumn{6}{|l|}{ General parenting } \\
\hline General Parenting & $\begin{array}{l}\text { Shortened version of the } \\
\text { Comprehensive General } \\
\text { Parenting Questionnaire } \\
\text { (CGPO) [98] }\end{array}$ & 3 & $\begin{array}{l}\text { Totally disagree / } \\
\text { totally agree }{ }^{1}\end{array}$ & & Parent \\
\hline Monitoring & & 1 & & & \\
\hline Consistent discipline & & 1 & & & \\
\hline Autonomy support & & 1 & & & \\
\hline $\begin{array}{l}\text { Children's behavioural prob- } \\
\text { lems and parents' self-efficacy }\end{array}$ & $\begin{array}{l}\text { Lifestyle Behaviour Checklist } \\
\text { (LBC) }[99,100]\end{array}$ & 50 & & & Parent \\
\hline Problem scale & & 25 & Not at all / very much ${ }^{2}$ & 0.9 & \\
\hline Confidence scale & & 25 & Grade $^{3}$ & 1.0 & \\
\hline \multicolumn{6}{|l|}{ Parenting practices } \\
\hline $\begin{array}{l}\text { Stimulation to be active and } \\
\text { to eat a healthy diet }\end{array}$ & [102] & 5 & $\begin{array}{l}\text { Totally disagree / } \\
\text { totally agree }{ }^{1}\end{array}$ & 0.6 & Parent \\
\hline $\begin{array}{l}\text { Parental role modelling- } \\
\text { healthy eating }\end{array}$ & $\begin{array}{l}\text { Comprehensive Feeding } \\
\text { Practices Questionnaire } \\
\text { (CFPQ) [103] }\end{array}$ & 4 & $\begin{array}{l}\text { Totally disagree / } \\
\text { totally agree }{ }^{1}\end{array}$ & 0.6 & Parent \\
\hline $\begin{array}{l}\text { Parental role modelling- } \\
\text { physical activity }\end{array}$ & $\begin{array}{l}\text { Home Environment Survey } \\
\text { (HES) [104] }\end{array}$ & 7 & $\begin{array}{l}\text { Totally disagree / } \\
\text { totally agree }^{1}\end{array}$ & 0.6 & Parent \\
\hline Food environment & CFPQ [103] & 2 & Never / always ${ }^{1}$ & 0.8 & Parent \\
\hline Parental policies & HES [104] & 2 & Never / always ${ }^{1}$ & 0.6 & Parent \\
\hline Emotional feeding & $\begin{array}{l}\text { Parental Feeding Style Ques- } \\
\text { tionnaire (PFSQ) [105] }\end{array}$ & 5 & Never / always ${ }^{1}$ & 0.8 & Parent \\
\hline Instrumental feeding & PFSQ [105] & 4 & Never / always ${ }^{1}$ & 0.7 & Parent \\
\hline Monitoring physical activity & $\begin{array}{l}\text { Physical activity }[102] \text { and } \\
\text { dietary intake with Child } \\
\text { Feeding Questionnaire (CFQ) } \\
{[101]}\end{array}$ & 6 & Never / always ${ }^{1}$ & 0.8 & Parent \\
\hline Covert control & Covert Control Scale [106] & 3 & Never / always ${ }^{1}$ & 0.7 & Parent \\
\hline Child control & CFPQ [103] & 5 & Never / always ${ }^{1}$ & 0.7 & Parent \\
\hline \multicolumn{6}{|l|}{ Motivation } \\
\hline $\begin{array}{l}\text { Motivation for physical } \\
\text { activity }\end{array}$ & $\begin{array}{l}\text { Behavioural Regulation of } \\
\text { Physical Activity in Children } \\
\text { (BRePAC) [154] }\end{array}$ & 18 & $\begin{array}{l}\text { Totally disagree / } \\
\text { totally agree }{ }^{1}\end{array}$ & & Child \\
\hline Amotivation & & 1 & & & \\
\hline External regulation & & 4 & & 0.6 & \\
\hline Introjected regulation & & 5 & & 0.5 & \\
\hline Controlled motivation & & & $\begin{array}{l}\text { Sum score }=(A M * 3)+ \\
(E X * 2)+(I J * 1)\end{array}$ & & \\
\hline
\end{tabular}


Table A3: continued

\begin{tabular}{|c|c|c|c|c|c|}
\hline Construct & Scale & Items & Answering options & $\begin{array}{l}\text { Cronbach's } \\
\text { alpha }\end{array}$ & Group \\
\hline Identified regulation & & 5 & & 0.8 & \\
\hline Intrinsic motivation & & 2 & & 0.7 & \\
\hline Autonomous motivation & & & $\begin{array}{l}\text { Sum score }=(I D * 2)+ \\
(I M * 3)\end{array}$ & & \\
\hline \multicolumn{6}{|l|}{ Behaviour-specific cognitions } \\
\hline Physical activity enjoyment & $\begin{array}{l}\text { Physical Activity Enjoyment } \\
\text { Scale (PACES) [115]; }\end{array}$ & 16 & $\begin{array}{l}\text { Totally disagree / } \\
\text { totally agree }{ }^{1}\end{array}$ & 0.9 & Child \\
\hline Habit strength & $\begin{array}{l}\text { Shortened version of the } \\
\text { Self-Report Habit Index } \\
\text { (SRHI) [117] }\end{array}$ & 9 & $\begin{array}{l}\text { Totally disagree / } \\
\text { totally agree }{ }^{1}\end{array}$ & & Child \\
\hline Sporting & & 2 & & 0.6 & \\
\hline Playing outside & & 2 & & 0.9 & \\
\hline Eating fruits & & 2 & & 0.7 & \\
\hline Self-efficacy & [116] & 21 & $\begin{array}{l}\text { Very difficult / totally } \\
\text { not difficult }{ }^{1}\end{array}$ & & Child \\
\hline Playing outside & & 13 & & 0.9 & \\
\hline Eating fruits & & 8 & & 0.6 & \\
\hline Attitude about eating fruits & & 7 & $\begin{array}{l}\text { Totally disagree / } \\
\text { totally agree }{ }^{1}\end{array}$ & 0.6 & Child \\
\hline \multicolumn{6}{|c|}{ Energy balance-related behaviours } \\
\hline Physical activity & [107] & 14 & $\begin{array}{l}\text { Not at all / } 3 \text { hours or } \\
\text { longer }{ }^{4}, \text { I don't know }\end{array}$ & & Parent \\
\hline Watching television & & 2 & & & \\
\hline Using a computer & & 2 & & & \\
\hline Playing outside & & 2 & & & \\
\hline Engaging in sports & & 2 & & & \\
\hline Walking or biking to school & & 2 & & & \\
\hline $\begin{array}{l}\text { Walking or biking during } \\
\text { leisure time }\end{array}$ & & 2 & & & \\
\hline Sports behaviour & & 1 & Days per week & & Child \\
\hline Dietary behaviours & [108] & 26 & $\begin{array}{l}\text { Days per week, fre- } \\
\text { quency per day }\end{array}$ & & Parent \\
\hline Breakfast & & 1 & & & \\
\hline Fruits & & 2 & & & \\
\hline Vegetables & & 4 & & & \\
\hline Water & & 2 & & & \\
\hline Sugar-sweetened beverages & & 2 & & & \\
\hline Diet beverages & & 2 & & & \\
\hline Fruit beverages & & 2 & & & \\
\hline Fruit juices & & 2 & & & \\
\hline Energy drinks & & 2 & & & \\
\hline Unhealthy snacks & & 7 & & 0.7 & \\
\hline
\end{tabular}


74 Chapter 4

Table A3: continued

\begin{tabular}{|c|c|c|c|c|c|}
\hline Construct & Scale & Items & Answering options & $\begin{array}{l}\text { Cronbach's } \\
\text { alpha }\end{array}$ & Group \\
\hline Sleeping behaviour & [109] & 6 & & & Parent \\
\hline Sleeping hours & & 2 & Hours per day & & \\
\hline Sleeping problems & & 4 & Never/always ${ }^{1}$ & & \\
\hline \multicolumn{6}{|l|}{ Quality of life and BMIz } \\
\hline Quality of life & $\begin{array}{l}\text { Impact of Weight on Quality } \\
\text { of Life (IWQOL)-Kids [110, } \\
111]\end{array}$ & 31 & Never/always ${ }^{1}$ & & Parent \\
\hline Physical comfort & & 6 & & 0.9 & \\
\hline Body esteem & & 9 & & 0.9 & \\
\hline Social life & & 6 & & 0.9 & \\
\hline Family relations & & 6 & & 0.7 & \\
\hline Total quality of life & & & Sum score & & \\
\hline Health of parent in general & & 1 & & & \\
\hline $\begin{array}{l}\text { Health of parent at that } \\
\text { moment }\end{array}$ & & 1 & & & \\
\hline Health of child in general & & 1 & & & \\
\hline $\begin{array}{l}\text { Health of child at that } \\
\text { moment }\end{array}$ & & 1 & & & \\
\hline$B M I z$ & {$[14,112,113]$} & 4 & $\begin{array}{l}\text { Centimetres, } \\
\text { kilo-grams, age, } \\
\text { ethnicity }\end{array}$ & & Parent \\
\hline
\end{tabular}

Notes: ${ }^{1}$ Five-point scale, ${ }^{2}$ seven-point scale, ${ }^{3}$ ten-point scale, ${ }^{4}$ six-point scale. 
4 



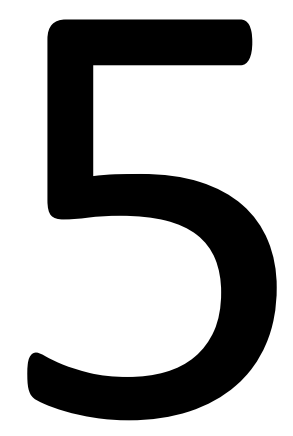

\section{The implementation of the Coaching on Life- style (CooL) intervention: lessons learnt}

Celeste E van Rinsum, Sanne MPL Gerards, Geert M Rutten, Madelon CG Johannesma, len $A M$ van de Goor, Stef PJ Kremers

Submitted

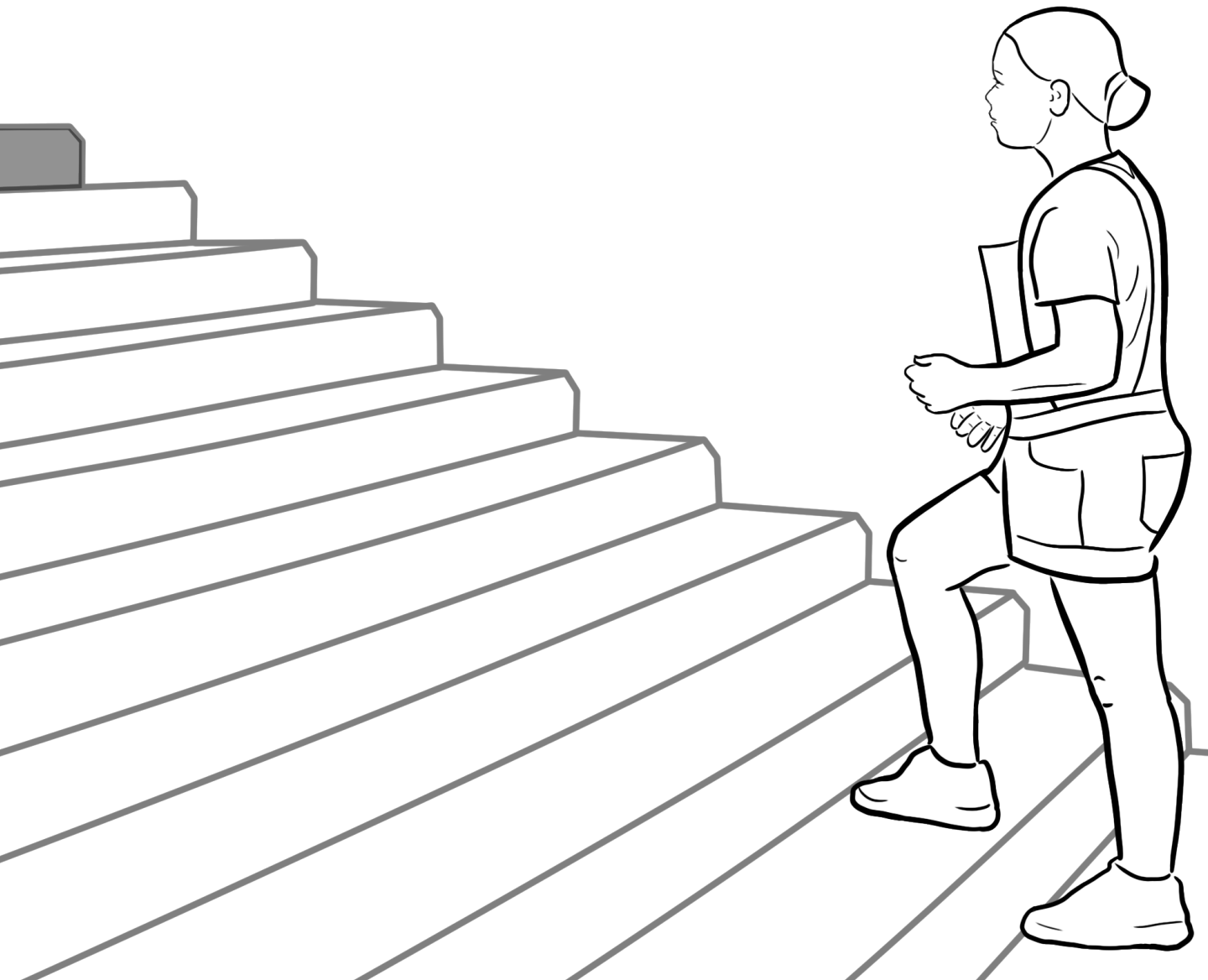




\section{ABSTRACT}

Background: Combined lifestyle interventions (CLIs) are designed to help people who are overweight or obese maintain a healthy new lifestyle. The CooL intervention is a CLI in the Netherlands, in which lifestyle coaches counsel adults and children (and/or their parents) who are obese or at high risk of obesity to achieve a sustained healthier lifestyle. The intervention consists of coaching on lifestyle in group and individual sessions, addressing the topics of physical activity, dietary behaviours, sleep, stress management and behavioural change. The aim of this study was to evaluate the implementation process of the Coaching on Lifestyle ( $\mathrm{CooL}$ ) intervention and its facilitating and impeding factors.

Methods: Mixed methods were used in this action-oriented study. Both quantitative (number of referrals, attendance lists of participants and questionnaires) and qualitative (group and individual interviews, observations, minutes and open questions) data were collected among participants, lifestyle coaches, project group members and other stakeholders. The Consolidated Framework for Implementation Research was used to analyse the data.

Results: CooL was evaluated by stakeholders and participants as an accessible and useful programme, because of its design and content and the lifestyle coaches' approach. However, stakeholders indicated that the lifestyle coaches need to become more familiar in the health care network and public sectors in the Netherlands. Lifestyle coaching is a novel profession and the added value of the lifestyle coach is not always acknowledged by all health care providers. Lifestyle coaches play a crucial role in ensuring the impact of CooL by actively networking, using clear communication materials and creating stakeholders' support and understanding.

Conclusion: The implementation process needs to be strengthened in terms of creating support for and providing clear information about lifestyle coaching. The CooL intervention was implemented in multiple regions, thanks to the efforts of many stakeholders. Lifestyle coaches should engage in networking activities and entrepreneurship to boost the implementation process. It takes considerable time for a lifestyle coach to become fully incorporated in primary care. 


\section{BACKGROUND}

An increasing proportion of the adult Dutch population is now overweight or obese (49.9\% and $14.2 \%$, respectively) [4]. The prevalence of overweight or obesity among children has also increased (13.3\% and $2.8 \%$, respectively). Combined lifestyle interventions (CLIs) help people who are overweight or obese change their physical activity level and dietary behaviours and maintain the new healthier lifestyle [121, 122]. However, many interventions have failed to translate research outcomes to real-world settings, due to unsuccessful or incomplete implementation $[155,156]$. Implementation of CLIs may benefit from process evaluation, as this provides insight into the implementation process. It also helps to understand the results of the intervention and the success factors influencing both the intervention and its implementation [157].

The implementation process of various types of $\mathrm{CLI}$ has been evaluated $[35,53,67$, $70-73,158]$. The results of many studies show too little multidisciplinary collaboration between important stakeholders, and professionals having insufficient skills and time to give participants the best possible guidance $[35,72,159,160]$. One important barrier stopping participants from attending CLIs was that health insurers refused to cover all costs [72]. Furthermore, previous studies have shown that long-term coaching is needed to maintain lifestyle changes $[73,83,121,161]$.

The Coaching on Lifestyle (CooL) intervention was developed based on previous research findings and addresses the most important bottlenecks. In this CLI, lifestyle coaches counsel, in separate groups, children and adults who are obese or at high risk of obesity. A lifestyle coach counsels a group of participants in the longer term, on average for 6 to 8 months. Lifestyle coaching encompasses integrating and addressing all major behavioural areas linked to obesity and lifestyle, i.e. physical activity, dietary behaviours, sleep, stress management and the umbrella topic of behavioural change. The essence of lifestyle coaching does not lie in its focus on the role of the professionals, nor in giving advice or directing participants. Instead it focuses on stimulating participants to take the lead and define their personal goals, guided by means of an autonomy-supportive coaching style of the lifestyle coaches. The lifestyle coach can act as a single point of contact for the participants regarding their lifestyle goals. The coach takes on the role of linchpin in the participants' care provider network. The intervention is reimbursed by health insurance companies and therefore free of charge for participants.

Since the trained lifestyle coach is a novel professional in Dutch primary care, a comprehensive implementation evaluation is required, taking into account factors that may be encountered during the implementation process. The research question of the current study was: How was the CooL intervention implemented and what were facilitating and impeding factors?

The results are described using the Consolidated Framework for Implementation Research (CFIR). This framework is a synthesis of existing implementation theories and it includes constructs of effective implementation [155]. These constructs are clustered in five domains, reflecting the characteristics of implementing an intervention. The CFIR 
was slightly modified to make it suitable to evaluate the CooL intervention (see Figure 1). The following key concepts of CFIR were operationalised: the unadapted and adapted intervention (CooL intervention), the process by which implementation is carried out (planning, engaging, executing, reflecting and evaluating), the inner setting (the organisation that implements the intervention: CooL organisation), the outer setting (participants, referrers and context) and the lifestyle coaches who carry out the intervention (defined in CFIR as 'individuals'). A successful implementation process focuses on the use of the intervention by the lifestyle coaches and the inner setting. Changes in the contextual outer setting are assumed to influence both the inner setting and the implementation process. It also shows that an intervention may evolve and be adapted to local preferences during the implementation process.

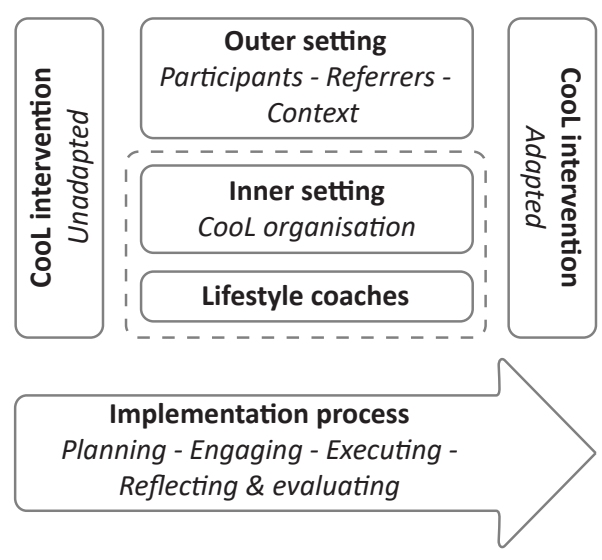

Figure 1: Schematic overview of the different implementation domains of the CooL intervention, based on the Consolidated Framework for Implementation Research (CFIR) [155]

\section{METHODS}

\section{CooL intervention}

The lifestyle coach leads the CooL programme, which consists of individual sessions and group sessions (see appendix Table 3 ). The programme targets both children (aged 4 years and older) and adults who are obese or at high risk of obesity. There are separate programmes for children, adolescents and adults. Children and adolescents are described as the same group, because of the small numbers in the programmes. Major themes are physical activity, dietary behaviours, sleep, stress management and behavioural change. The aim is to change the lifestyle pattern of the participants in a stepwise fashion and to achieve sustainable lifestyle change. If required, after the basic programme each participant can be included in one of additional programmes, namely the relapse prevention programme (group and individual sessions) or the additional programme (only individual sessions). A total of 13 lifestyle coaches, who had completed a post-graduate training course at the Dutch Academy for Lifestyle and Health (AVLEG), were involved in the pilot programme. More information about the content of the 
programme can be found elsewhere, as well as the methods, techniques and working approaches used in the intervention [123, 162].

The pilot started in two regions in the southern part of the Netherlands with the programme for adults (Regions 1 and 2) and in two other regions for the children's programme Regions 3 and 4) in 2014. During the subsequent pilot period, more regions were added. At the end of the study period, the adult programme was implemented in five regions (Regions 1 and 2 plus Regions 5, 6 and 7) and four children's regions (Regions 3 and 4 plus Regions 2 and 5). The adult participants were mostly referred to CooL by their general practitioners or their practice nurses. The children were mostly referred by the Youth Health Care (YHC) service.

In each region, a project group was responsible for the local implementation of the intervention. These project groups consisted of the central project leader, the lifestyle coaches involved, a coordinator from the local 'health care group' (i.e. coordinating organisation for primary care providers) or from the public health services, a representative of the local sports organisation and a care purchasing agent of the health insurance company. The project leader's role changed during the last year of the study, from external change agent to main researcher (CvR). In addition to the project groups, there was a steering group which was responsible for general decisions about the programme, its implementation and the evaluation study. During peer feedback meetings with their supervisor, the lifestyle coaches discussed problems that occurred in the implementation, shared best practices and learned from each other's experiences.

\section{CooL study}

The study protocol of the CooL study has been published and presents a detailed description of the study and the methods [123]. The lifestyle changes achieved among CooL participants have been reported in an earlier publication [162]. Briefly, the results showed positive and sustained changes among adults regarding motivation, behaviour-specific cognitions, lifestyle behaviours, quality of life and weight. Among children and their parents, few improvements were found regarding behaviours and quality of life.

The present paper describes the implementation process. Both quantitative and qualitative data were used (see Table 1 for an overview). Since the overall study was designed as an action-oriented study, the research team was closely allied with the implementation process. Since the main researcher participated in all organisational meetings, this enabled her to observe and simultaneously support the implementation process. The CFIR framework was used to analyse and cluster the data. Data collection took place between 1 May 2014 and 1 April 2017.

The quantitative measures included the number of referrals, attendance lists of participants, questionnaires for participants about their satisfaction with the intervention and the guidance provided by their lifestyle coach, and questionnaires for lifestyle coaches to assess their competences. 
The qualitative methods consisted of group and individual interviews with the participants, lifestyle coaches and other stakeholders, observations and minutes of group sessions and meetings, and a questionnaire with additional process questions. The interview structures were based on various implementation theories [156, 163], adapted to the CooL intervention.

Table 1: Study components and methods used, for each domain of the CFIR framework

\begin{tabular}{|c|c|c|c|c|}
\hline Domain & Evaluation components & Method & Target group & $\mathbf{N}$ \\
\hline \multirow[t]{2}{*}{ Intervention } & $\begin{array}{l}\text { Evolution of the pro- } \\
\text { gramme }\end{array}$ & Observations & Peer feedback meetings & 13 \\
\hline & $\begin{array}{l}\text { Programme fidelity: } \\
\text { executed as intended }\end{array}$ & Interviews & Lifestyle coaches & 12 \\
\hline \multirow[t]{3}{*}{ Lifestyle coaches } & $\begin{array}{l}\text { Competences of lifestyle } \\
\text { coaches }\end{array}$ & Questionnaire & Lifestyle coaches & 13 \\
\hline & & Questionnaire & Participants & 187 \\
\hline & Tasks of lifestyle coaches & Questionnaire & $\begin{array}{l}\text { Referrers, project group mem- } \\
\text { bers, lifestyle coaches and local } \\
\text { parties }\end{array}$ & 129 \\
\hline Inner setting & $\begin{array}{l}\text { Organisations in the } \\
\text { various CooL regions }\end{array}$ & $\begin{array}{l}\text { Weekly telephone } \\
\text { meetings }\end{array}$ & Project leader & 72 \\
\hline \multirow[t]{2}{*}{ Outer setting } & $\begin{array}{l}\text { Number of referrals and } \\
\text { attendance rates }\end{array}$ & $\begin{array}{l}\text { Registration lists } \\
\text { and attendance } \\
\text { lists }\end{array}$ & Lifestyle coaches & 13 \\
\hline & $\begin{array}{l}\text { Involvement and opinion } \\
\text { of stakeholders }\end{array}$ & Questionnaire & $\begin{array}{l}\text { Referrers, project group mem- } \\
\text { bers, lifestyle coaches and local } \\
\text { parties }\end{array}$ & 129 \\
\hline \multirow[t]{12}{*}{ Process } & \multirow{6}{*}{$\begin{array}{l}\text { Experiences with the } \\
\text { programme: satisfaction }\end{array}$} & Questionnaire & Participants & 187 \\
\hline & & Group interviews & Participants & 6 \\
\hline & & Interviews & Participants & 4 \\
\hline & & & Referrers & 52 \\
\hline & & & Project group members & 14 \\
\hline & & & Lifestyle coaches & 12 \\
\hline & \multirow{6}{*}{$\begin{array}{l}\text { Facilitating and impeding } \\
\text { factors for successful } \\
\text { implementation during } \\
\text { different implementation } \\
\text { phases }\end{array}$} & Interviews & Referrers & 52 \\
\hline & & & Project group members & 14 \\
\hline & & & Lifestyle coaches & 12 \\
\hline & & $\begin{array}{l}\text { Observations and } \\
\text { minutes }\end{array}$ & $\begin{array}{l}\text { Project \& steering group \& } \\
\text { peer feedback meetings }\end{array}$ & 107 \\
\hline & & & Group sessions & 28 \\
\hline & & Questionnaire & $\begin{array}{l}\text { Referrers, project group mem- } \\
\text { bers, lifestyle coaches and local } \\
\text { parties }\end{array}$ & 129 \\
\hline
\end{tabular}

Note: $\mathrm{N}=$ number of respondents. 


\section{RESULTS}

The results are described for each of the domains presented in Figure 1, based on the CFIR framework. The facilitating and impeding factors are outlined in each domain and are listed in Table 2. For each domain, multiple perspectives are presented, such as those of the lifestyle coaches, project group members, referrers and participants.

\section{Unadapted intervention}

At the start of the implementation process, the content of the various programmes was not designed or protocolled in full detail. This left the lifestyle coaches the opportunity to fill in the contents according to their own preferred working methods. Major topics were established in advance as key elements of the programme (physical activity, dietary behaviours, sleep, stress management and behavioural change). The lifestyle coaches were trained to develop their own programme, based on evidence-based behaviour change approaches, their general coaching styles, specific coaching strategies and knowledge gained in their training course. The coaches in the regions that were the first to start designed their own programme content and discussed this with the others during peer feedback meetings. The lifestyle coaches shared all formats with the other coaches.

\section{Lifestyle coaches}

The questionnaire regarding the lifestyle coaches' competences showed that the coaches were significantly more engaged in their work than average Dutch employees [164]. Empathising with others was their strongest competence, which they also indicated as the most important competence for a lifestyle coach. The coaches evaluated entrepreneurship as their weakest competence, but at the same time they thought this was the least important competence to have as a lifestyle coach. The majority of lifestyle coaches appeared to lack these additional skills during the pilot, which impeded the effectiveness of their coaching. During the interviews, the coaches indicated that coaching skills and empathic skills are necessary. They also mentioned that it is necessary to be inquisitive and to keep up to date with the most recent scientific insights. Some of the lifestyle coaches admitted that they did not feel confident at first about counselling a whole group, but that they had soon gained confidence. In addition, project group members noticed that the coaches needed to be flexible about their working hours, as some participants could only be seen in the evenings, for example. One of the lifestyle coaches resigned because travelling took too much of her time.

\section{Stakeholders' perspective}

The stakeholders, including referrers, project group members, lifestyle coaches and local parties most commonly defined the lifestyle coaches' tasks as guiding participants towards a sustained healthier lifestyle, addressing all lifestyle themes (such as physical activity and stress management). When asked for more details, they explained they were referring to creating awareness, transferring knowledge, providing information and advice, intrinsically motivating participants, signalling problems, helping partici- 
Table 2: Facilitating and impeding factors for each domain

\begin{tabular}{|c|c|c|}
\hline Domain & Facilitating factors & Impeding factors \\
\hline Lifestyle coaches & $\begin{array}{l}\text { - High level of work engagement } \\
\text { - } \text { Empathising with others } \\
\text { - } \text { the participants } \\
\text { - High involvement } \\
\text { - Great enthusiasm } \\
\text { - } \text { Openness } \\
\text { - } \text { Pupporting instead of directing } \\
\text { - Confidence in participants } \\
\text { - Knowledge and skills regarding sys- } \\
\text { tematic behaviour change }\end{array}$ & $\begin{array}{l}\text { - Lack of entrepreneurship } \\
\text { - Lack of networking skills } \\
\text { - Not using the professional network } \\
\text { for referring }\end{array}$ \\
\hline Inner setting & $\begin{array}{l}\text { - Having project groups } \\
\text { - Locations in the neighbourhood } \\
\text { - Support from the health care centre } \\
\text { - } \text { Cooperation between the LSCs }\end{array}$ & $\begin{array}{l}\text { - No appropriate financial compensa- } \\
\text { tion for lifestyle coaches } \\
\text { - Too many unpaid administrative tasks } \\
\text { for lifestyle coaches }\end{array}$ \\
\hline Outer setting & & \\
\hline Participants & $\begin{array}{l}\text { - Low drop-out rates } \\
\text { - Intrinsic motivation to change before } \\
\text { the start } \\
\text { - High self-efficacy to change }\end{array}$ & $\begin{array}{l}\text { - History of multiple failures in trying } \\
\text { to lose weight } \\
\text { - Having other more important prob- } \\
\text { lems decreases motivation } \\
\text { - } \text { Financial problems } \\
\text { - Sense of not fitting in with the group } \\
\text { - Unsupportive parents regarding } \\
\text { changing their child's lifestyle }\end{array}$ \\
\hline Referrers & $\begin{array}{l}\text { - Personal motivation of referrers } \\
\text { - Referrers' knowledge of and experi- } \\
\text { ence with lifestyle coaching and the } \\
\text { coaches }\end{array}$ & $\begin{array}{l}\text { - Perceived lack of time or priority to } \\
\text { be involved in the programme } \\
\text { - Some referrers knew too little about } \\
\text { the programme }\end{array}$ \\
\hline Context & $\begin{array}{l}\text { - Expected future coverage of CLIs by } \\
\text { health insurance } \\
\text { - Collaborating with other partners } \\
\text { and different disciplines } \\
\text { - Increased familiarity with the lifestyle } \\
\text { coaches and their role }\end{array}$ & $\begin{array}{l}\text { Health care professional's unaware- } \\
\text { ness about their role in lifestyle } \\
\text { change }\end{array}$ \\
\hline \multicolumn{3}{|c|}{ Implementation process } \\
\hline Planning & $\begin{array}{l}\text { - Involvement of stakeholders in } \\
\text { project groups }\end{array}$ & $\begin{array}{l}\text { - Too little time for implementation to } \\
\text { create support among the referrers }\end{array}$ \\
\hline Engaging & $\begin{array}{l}\text { - } \text { Creating support } \\
\text { - Kick-off meetings } \\
\text { - Protocols for lifestyle coaches and } \\
\text { referrers }\end{array}$ & $\begin{array}{l}\text { - Not having the logistics organised at } \\
\text { the start of the implementation }\end{array}$ \\
\hline
\end{tabular}


Table 2: continued

\begin{tabular}{|c|c|c|}
\hline Domain & Facilitating factors & Impeding factors \\
\hline Executing & $\begin{array}{l}\text { Effective communication and collabo- } \\
\text { ration between lifestyle coaches and } \\
\text { referrers } \\
\text { - Attending more meetings to inform } \\
\text { the referrers } \\
\text { - Articles in local newspapers }\end{array}$ & $\begin{array}{l}\text { - Time investment for lifestyle coaches, } \\
\text { stakeholders and participants } \\
\text { - Too few personal contacts with } \\
\text { referrers } \\
\text { - Lack of clear communication mate- } \\
\text { rials }\end{array}$ \\
\hline $\begin{array}{l}\text { Reflecting and } \\
\text { evaluating }\end{array}$ & $\begin{array}{l}\text { - Most participants were satisfied } \\
\text { - Ensuring well-organised precondi- } \\
\text { tions } \\
\text { - Having suitable manuals for new } \\
\text { lifestyle coaches }\end{array}$ & $\begin{array}{l}\text { - Too heterogeneous groups and large } \\
\text { differences between participants } \\
\text { - Too much time between contact } \\
\text { moments, and between registration } \\
\text { and start of the group } \\
\text { - Too few individual coaching sessions } \\
\text { (for children) } \\
\text { - No ambassador in every region }\end{array}$ \\
\hline CooL intervention & $\begin{array}{l}\text { - Frequent contacts over a period of } \\
\text { - Optix months } \\
\text { and group sessions } \\
\text { - Not only focusing on nutrition, but } \\
\text { multiple themes including stress and } \\
\text { sleep } \\
\text { - Learning from peers } \\
\text { - Whole family takes part in the chil- } \\
\text { - } \text { dren's programme } \\
\text { - Participant-centred approach } \\
\text { - Positive approach aimed at increas- } \\
\text { - } \text { ing autonomous motivation } \\
\text { - imowledge transfer and practical } \\
\text { - Approach tailored to the participants' } \\
\text { - } \text { needs } \\
\text { - Elexibility in design and content } \\
\text { No charge for participants }\end{array}$ & $\begin{array}{l}\text { - Inadequate time slots for group } \\
\text { sessions } \\
\text { - Strict inclusion criteria } \\
\text { - Participant materials with too much } \\
\text { text } \\
\text { - Materials not suitable for non-Dutch } \\
\text { speaking persons }\end{array}$ \\
\hline
\end{tabular}


pants set realistic goals, supporting, helping participants to learn new skills, and improving self-management. They also emphasised the importance of having a positive approach, monitoring the process, tailoring the programme and finding a suitable form of physical activity together with each participant. Some of the stakeholders mentioned that lifestyle coaches' tasks also included communicating with referrers, providing them with feedback, referring participants to other professionals and networking with stakeholders.

\section{Inner setting}

\section{Financial organisation}

The lifestyle coaches, as well as the project group members, had to invest time and money at the start. The fees for each individual participant, paid by the health insurers, did not cover all the costs for the lifestyle coaches. The meeting time and contact time with absent participants were not included in these fees, nor was the time needed to design the detailed content of the programme and complete portfolios and plans of action. At least eight participants per group were required to break even and make it viable to start a group. For the children's groups, it was not easy to make up a group large enough to cover the costs.

\section{Organisation within regions}

In most of the regions, one or two lifestyle coaches were assigned, in which case they both counselled their own groups. In Region 6 the two lifestyle coaches divided the tasks: one coach was responsible for the coaching and the other for the networking and registration of participants. They both experienced this as a good and pleasant task division. In Region 5 the lifestyle coach received administrative support from the local health care group, which helped her considerably.

\section{Locations}

It was a barrier for participants when the meeting location was not in their immediate neighbourhood. Therefore, the group sessions were held in locations as close to the participants residences as possible, and in rent-free or cheap locations, to minimise the intervention costs. The chosen locations included meeting rooms of the health care groups or the health insurance company, community centres and schools. The children's lifestyle coach of Region 2 was sometimes present at the location of the YHC referrer. This gave the participants the opportunity to immediately plan an intake session.

\section{Outer setting}

\section{Participants}

During the study period, 494 adults were referred to the CooL intervention, 358 of whom actually started the intervention. A total of 66 adults (18\%) dropped out during the programme. The number of referrals of children and adolescents was 192, 106 of whom started the programme, and $22(21 \%)$ children dropped out. 


\section{Participants' characteristics}

Among the CooL participants, adults had an average BMI of 36.1, while the children had an average BMI z-score of 2.3. The self-reported educational level of the majority of the adults and the children's parents was low or intermediate. The study population had tried to lose weight before, but were unable to maintain this weight loss for more than one year. Participants with a low autonomous motivation were more likely to drop out of the programme. The lifestyle coaches noticed during the implementation that the participant's motivation should preferably be checked at the intake session, which made the operationalisation of the inclusion criteria stricter as the pilot progressed. Participants with a higher autonomous motivation were more conscious of their unhealthy behaviours and felt more responsible for them. Overweight parents were less motivated to participate in the programme with their children, compared to parents with a normal weight. In the baseline questionnaire, $15 \%$ of the parents answered that it had actually come as a surprise to them that their child's weight was a matter of concern.

\section{Reasons and criteria for not starting}

There were several reasons why potential participants decided not to attend the programme. The most frequently mentioned reason was lack of motivation. This appeared to be more often the case for participants with multiple problems, such as diseases, financial problems or mental problems. Another important impeding factor was that some participants did not like to participate in a group. Most children or their parents showed a need for more individual guidance, which was sometimes provided by the lifestyle coaches.

The most common criticism among lifestyle coaches and referrers was the strictness of the inclusion criteria for CooL, particularly for children. When children were obese at a young age, this usually meant there were more problems in the family. In such multi-problem families, lifestyle change is typically not their first priority. Lifestyle coaches reported a preference for a less strict inclusion criterion for weight status.

\section{Referrers}

Referrers reported that patients were hard to reach and to motivate for participation in the programme. Furthermore, they saw multiple barriers to taking part in the intervention, especially at the beginning. They were under great pressure of time, and they felt that there was no time or priority for referring patients to the programme. Their awareness of the intervention decreased over time, because they were not referring to it on a regular basis. Professionals who saw the advantage of the intervention and had a passion for prevention referred more patients. It depended on the region and the lifestyle coach's place within the care network whether they received more referrals and support from the referrers. Considerable time went by before referrers became aware of the positive results of the intervention and realised the benefits and relevance of the CooL programme.

\section{Context}

The goal of the pilot was to evaluate and further develop the implementation process, 
with the ultimate aim of arriving at an optimal design for the systematic uptake of CooL in the Dutch health care system. At the time the pilot started, in 2014, obesity care was not a common theme to discuss during consultations in primary care [29]. General practitioners were insufficiently trained to discuss lifestyle with their patients [165]. Health care professionals typically applied a mono-disciplinary approach to their patients, for example physiotherapists mainly tried to improve their musculoskeletal system [166]. Care for patients was fragmented. The idea that obesity should be addressed in an integrated approach did gain some ground, but at a very slow pace [14]. At the local level, the implementation of CooL started in regions where covenants, connections and other arrangements among the care providers already existed and prevention was already on the agenda more explicitly than in many other regions in the country.

\section{The central role of lifestyle coaches}

In the course of the process, the lifestyle coaches' role as linchpins in obesity care appeared crucial. If lifestyle coaches were part of relatively dense networks, this meant that participants were more likely to be referred to these coaches. In any case, referral to CooL was suboptimal and lifestyle coaches should become more visible as an important stakeholder in obesity care.

\section{Changed context}

Currently, health care professionals and policy makers have become more aware of the importance of lifestyle behaviour for health outcomes [165]. Integrated approaches to the prevention of chronic diseases have become more common over time. In the course of the implementation process it was becoming clearer that CLIs would be included in health insurance policies in the Netherlands from 2019 onwards [166]. This had a positive influence on the motivation of the lifestyle coaches, referrers and other stakeholders. The lifestyle coaches invested more time in describing and detailing the adjusted intervention contents than in the early stages of the pilot. The referrers increasingly perceived CooL as a permanent referral option instead of just another project.

\section{Stakeholders' contributions}

Most stakeholders (66\%) saw themselves as contributing relatively little to the programme; although some stakeholders were relatively active (24\%) and a small proportion contributed greatly (10\%). The most commonly mentioned reasons to participate were: improving the participants' health (82\%); the sense that the programme was a good initiative (70\%); collaboration with other disciplines/organisations (33\%); and referring people (28\%). Furthermore, most of them fully agreed (on a 5-point Likert scale) with the statements that the lifestyle coach represented a useful addition to the health care network (46\%) and that the lifestyle coaching programme was a valuable innovation (48\%). They were a bit more reserved about the statement that there was a need for the lifestyle coaching programme in the health care system (38\%).

\section{Implementation process}

\section{Planning}

The implementation started with the programme for adults, and involved a small se- 
lection of interested general practices. Meanwhile, the sample size was calculated and lifestyle coaches were spread over the regions. When the number of referrals was found to be low, the inclusion period was extended and all general practices in each region were invited to refer patients to CooL. Some practices (2\%) declined this invitation, as they did not want to invest time.

\section{Engaging}

The lifestyle coaches used kick-off meetings and information provision to referrers during group or individual meetings to try and create more support among the referrers. The referrers received an information package with a flyer for patients and a referral protocol, which presented information on how to sign up patients and what was expected from them. The lifestyle coaches had also been informed about the referral process and the execution of the intervention by means of a protocol.

In the beginning of the pilot programme, the logistics of the intervention had not yet been fully organised at the start of the intervention's implementation. The contacts with stakeholders had already been established before the information was prepared and the programme was finalised. On the one hand, this meant that the information was distributed in phases. On the other hand, the stakeholders could already contribute to the implementation process.

When one of the two programmes had already been implemented in a particular region, the chances were greater that the second programme would be implemented as well (in most cases the children's programme followed the adult programme).

\section{Executing}

The referrers indicated during the interviews that they wanted to know who the lifestyle coaches were, and the lifestyle coaches noticed that the referrers had many practical questions. Project groups members therefore pointed out that personal contact was very important to increase the referrers' motivation. This demanded a lot of time investment on the part of the lifestyle coaches. Furthermore, the question remained to what extent the referrers were aware of the programme and the referral process. In each region, newsletters were sent by the health care group or public health services, presenting the most important information and updates. Referrers and lifestyle coaches reported that the patient flyer was too complicated and that it lacked a clear and understandable description of the intervention. Their comments were used to improve the communication and information for referrers and potential participants.

\section{Reflecting and evaluating}

Based on attendance lists, it appeared that the adult participants attended on average $5.3( \pm 2.3)$ group sessions and $2.9( \pm 0.9)$ hours of individual coaching. Their total programme covered $188.4( \pm 89.4)$ days. Children, adolescents and their parents participated in the CooL programme for 229.4 ( \pm 128.5$)$ days. They attended $3.8( \pm 2.6)$ group sessions and had $4.2( \pm 1.9)$ hours of individual sessions. 


\section{Evaluation by the participants}

On average, the participants were satisfied with the programme, the group sessions, individual sessions and the work of the lifestyle coach. The participants rated the programme at about 8 out of 10 (adults: 8.6 ; parents: 8.5 ; children: 7.8 ). There were a few exceptions. For example, some participants had expected a stricter approach, in which they were told how much to exercise and what to eat. This expectation conflicted directly with the nature of lifestyle coaching, in which the participant is supposed to take the leading role and is in charge of their own goals and corresponding actions.

Most participants perceived the combination of group and individual sessions as pleasant. The individual guidance enabled them to discuss personal problems. The group dynamics in the group sessions linked them to fellow sufferers and familiar problems were discussed. However, some participants reported in the questionnaire that they felt a need for a more personal approach. This remark typically came from participants in larger groups (often larger than ten members) and from participants in groups with persons with special needs. This made it harder to give enough personal time and space to all the group members.

In addition, some of the participants wanted to have more practical assignments, for example more assignments with pictures, audio-visual tools, digital materials and less text. Finally, the participants mentioned in the early stage of the pilot programme that they needed refresher sessions to better maintain their changed behaviours.

\section{Lifestyle coaches' perspective}

According to the lifestyle coaches, the ideal group size was about ten to twelve participants. In reality, the groups were often smaller, since some of the participants did not always attend. Moreover, it was hard to get enough people for the groups, which made the time between registration and the start of the programme rather long for some participants. It also led to mixed group compositions, with different ages and cognitive skills. Participants could not identify themselves with the other group members when the differences between them were large. The lifestyle coaches argued that it would be desirable to work with more homogeneous groups, so they could easily adjust the content of the programme to the level of the group. The participants could then learn more from each other and the group process would improve. Furthermore, the lifestyle coaches perceived the home visits for children and their parents as valuable, as it made their daily lives and behavioural patterns more visible and could be discussed more easily.

\section{Project groups}

The implementation process was discussed at every monthly project group meeting in each region. If the implementation was not yet successful, new actions were instigated to improve the information available among the stakeholders. In the early stage of the pilot, the project group members noticed that the division of roles and expectations was not clear to all of them. In some cases, it was unclear who was responsible for which tasks, such as arranging the location for the group sessions. Another observation was that the project leaders were often geographically far removed from the pilot re- 
gion and that they were not familiar with the stakeholders in the networks.

\section{Adapted intervention}

The core components of the programme were the sessions with their fixed themes (see Table 3 in the appendix), as well as appointing one lifestyle coach to each group. The themes were sometimes presented in a different order and the contents were adapted to meet the needs of the group. The exact content and the practical exercises were part of the 'adaptable periphery' of the intervention protocol. In the unadapted intervention, the lifestyle coaches started with their own custom-made content and exercises. During the course of the implementation process, the lifestyle coaches shaped and finalised the content based on their professional knowledge, their expertise, feedback from the participants, evaluation sessions with other lifestyle coaches and interim findings from the current action-oriented study. They exchanged practical exercises and assignments for the group sessions, and discussed difficult cases of participants and their own coaching methods with each other during peer feedback meetings. Gradually during the study period, they compared their differences and combined their best practices into a final programme format. A document was produced which described the goals and multiple examples of exercises for each group session, to support lifestyle coaches in designing sessions for their own groups and in their own context. When the intervention document was being drafted, the coaches were invited to substantiate the programme with underlying theories, strategies and applications [168].

\section{DISCUSSION}

The aim of this study was to examine the implementation process of the CooL intervention and its facilitating and impeding factors. We found that the principles that contributed most to the successful implementation of CooL were: having one professional (the lifestyle coach) for multiple lifestyle-related themes, offering a combination of group and individual sessions for adults, the family approach for children, a high frequency of sessions, easy accessibility for participants and the fact that the programme was offered free of charge. Impeding aspects for the intervention were the strict inclusion criteria and small group sizes. Crucial factors for lifestyle coaches included empathising with the participants and having a high work engagement. Impeding factors for the lifestyle coaches were a lack of networking skills and entrepreneurship. The most important facilitating factors for the inner setting (i.e. the CooL organisation) were the project groups and close proximity of the intervention location. CooL participants were more likely to participate when they had a strong intrinsic motivation to change. Factors that make it less likely for people to participate or to complete the programme included not fitting in with the group and having financial constraints. As regards the outer setting, the contacts between lifestyle coaches and their network were crucial. Greater familiarity with and a positive attitude towards the lifestyle coaches' role among the stakeholders were necessary for effective implementation. It helped if the coaches were able to strengthen their network to ensure optimal referral of participants. 
Effective implementation starts by creating support among stakeholders, such as referrers. Since the role of lifestyle coach is a new one in the health care system, it has not yet become very familiar. Therefore, we recommend that the central role of the lifestyle coach is more clearly positioned in the integrated approach to obesity. Above all, personal contacts are crucial, and intensive collaboration between coaches and other professionals will help increase their familiarity and trust among other network members [169]. A trend towards increased motivation of referrers was observed towards the end of the pilot period.

If more stakeholders support the intervention, they will probably contribute more effectively to accelerating the recruitment of participants. Slow recruitment processes have also been found in other studies $[85,158]$ and this remains an issue of concern. An important cause of the low number of referrals was the lack of clear communication materials for the referrers. In combination with the low frequency of personal contacts with referrers, this meant that not all referrers had sufficient knowledge about the intervention, about their specific role in the process and about how to refer patients. More contacts and better information could probably take away the barriers from the referrers, such as the time investment required for referring [170]. Since general practitioners are not trained to assess a patient's motivation, they should be assisted by the lifestyle coaches to make this assessment [27]. The fact that the costs of CLIs are expected to be covered by health insurance may help to institutionalise the referral process [16]. If lifestyle coaches informed the referrers more effectively about the participants' progress, referrers might take a more positive view of the programme $[171,172]$.

Investment in the contacts among the stakeholders could make the relationships sustainable, with help from an 'ambassador' or a broker [160, 173]. Such an ambassador should be in close contact with the stakeholders in the region and can probably take over some of the networking and entrepreneurial tasks from the lifestyle coach, if this person is not the lifestyle coach. The role could be filled by the lifestyle coach, a local project leader, someone from a central organisation (e.g. a health care group) or a central person in the network of public health and health care (e.g. a health broker [174]).

Extensive preparation and implementation time is needed to create support among the stakeholders and to create an optimal intervention context. This is often underestimated. Depending on the characteristics of the context, it can take up to a few years [175].

The autonomous motivation to change has been shown to be crucial for the attendance of participants as well as for intervention effects $[160,176]$. People differ in their readiness to change their unhealthy behaviour. This depends on their previous experiences [177] and the extent to which they experience the negative effects of their current behaviour. The lifestyle coach uses methods like motivational interviewing to try increase this autonomous motivation [178]. 


\section{Strengths and limitations of the study}

Strengths of this study were its action-oriented approach, the real-world setting in different regions and the use of several implementation process methods and instruments. Thanks to the action-oriented approach, the collaboration between the lifestyle coaches and the researchers was good and the implementation process could be closely followed and improved when needed. Implementing an intervention in a real-world setting is always complex, due to contextual and systemic processes [179]. But the chances of achieving sustainability of the CooL intervention and its nation-wide dissemination are probably greater than if the pilot had been accompanied by a controlled trial [180]. Another added value of this study was the use of mixed methods, which gave us information from different points of view, viz. those of the stakeholders, lifestyle coaches, researchers and participants.

The lifestyle coaches constantly adjusted and adapted the CooL programme to the participants' needs during the study period. They worked in their own way, but used the same themes, general principles and way of thinking. These programme changes and the different ways in which it was executed made it impossible to measure the programme fidelity among the lifestyle coaches. This may be viewed as a limitation, but in line with basic assumptions underlying the CFIR for evaluating interventions in complex systems [64, 181], we postulate that adaptation is desirable and promoting complete programme fidelity may even be harmful (Schaap et al., unpublished observations). A limitation of this study is that the data were not analysed with qualitative software programmes, such as Nvivo. The amount of data and the different types of qualitative data (ranging from observations and minutes of meetings to semi-structured interviews) prevented us from adopting a computerised approach to the analyses. Instead, paper-and-pen methodologies were used, while applying a coding system based on our research framework.

\section{Conclusions}

The aim of this study was to examine the implementation process of the CooL intervention and its facilitating and impeding factors. A substantial number of barriers have been overcome and promising opportunities have arisen for integrating lifestyle coaching in a broader approach, to bridge the gap between prevention and treatment of chronic diseases. However, the dissemination process of CooL still needs to be improved further. Networking activities should be intensified and the contents of the intervention continuously improved to fit both the inner and outer implementation settings. It will take time before the lifestyle coaches have become accepted as valuable professionals who bridge the gap between the public health sector and health care settings. We expect our recommendations to be helpful in improving the dissemination and monitoring of combined lifestyle interventions. 
94 Chapter 5

\section{APPENDIX ADDITIONAL TABLE}

Table 3: Number of sessions per target group and per programme, and themes per group session

\begin{tabular}{|c|c|c|c|}
\hline Components & Children & Adolescents & Adults \\
\hline Basic programme & 9 to 10 months & 9 to 10 months & 7.5 months \\
\hline Individual sessions & $\begin{array}{l}\text { Maximum } 7 \text { hours }(10 x) \\
\text { at home }\end{array}$ & $\begin{array}{l}\text { Maximum } 7 \text { hours }(10 x) \\
\text { at home }\end{array}$ & $\begin{array}{l}2 \times 60 \text { minutes } \& 2 \times 45 \\
\text { minutes }\end{array}$ \\
\hline \multirow[t]{3}{*}{ Group sessions } & $\begin{array}{l}8 \times 90 \text { minutes for the } \\
\text { parents }\end{array}$ & $\begin{array}{l}5 \times 90 \text { minutes for ado- } \\
\text { lescents }\end{array}$ & $8 \times 90$ minutes \\
\hline & & $\begin{array}{l}1 \times 90 \text { minutes for } \\
\text { parents }\end{array}$ & \\
\hline & & $\begin{array}{l}\text { 2x } 90 \text { minutes for ado- } \\
\text { lescents and parents }\end{array}$ & \\
\hline 1 & $\begin{array}{l}\text { Awareness and be- } \\
\text { haviour change }\end{array}$ & $\begin{array}{l}\text { Awareness and be- } \\
\text { haviour change }\end{array}$ & $\begin{array}{l}\text { Awareness and be- } \\
\text { haviour change }\end{array}$ \\
\hline 2 & Physical activity & $\begin{array}{l}\text { Acting as a role model } \\
\text { (only for parents) }\end{array}$ & Physical activity \\
\hline 3 & Nutrition & Physical activity & $\begin{array}{l}\text { Structured eating } \\
\text { patterns }\end{array}$ \\
\hline 4 & $\begin{array}{l}\text { Setting boundaries and } \\
\text { rewarding }\end{array}$ & $\begin{array}{l}\text { Nutrition (including } \\
\text { parents) }\end{array}$ & Sleep, relaxing, stresses \\
\hline 5 & Acting as a role model & Snacking & Time management \\
\hline 6 & Sleep, relaxing, stresses & Sleep and relaxing & Pitfalls \\
\hline 7 & Pitfalls and planning & Stresses and pitfalls & Relapse prevention \\
\hline 8 & $\begin{array}{l}\text { Self-regulation for the } \\
\text { family }\end{array}$ & $\begin{array}{l}\text { Self-regulation for } \\
\text { the family (including } \\
\text { parents) }\end{array}$ & Self-regulation \\
\hline $\begin{array}{l}\text { Relapse prevention } \\
\text { programme }\end{array}$ & $\begin{array}{l}\text { Same number of ses- } \\
\text { sions as basic interven- } \\
\text { tion, spread over } 2 \text { years }\end{array}$ & $\begin{array}{l}\text { Same number of ses- } \\
\text { sions as basic interven- } \\
\text { tion, spread over } 2 \text { years }\end{array}$ & $\begin{array}{l}\text { Same number of ses- } \\
\text { sions as basic interven- } \\
\text { tion, spread over } 2 \text { years }\end{array}$ \\
\hline Additional programme & - & - & $\begin{array}{l}10 \times 30 \text { minutes individu- } \\
\text { al sessions }\end{array}$ \\
\hline
\end{tabular}


5 


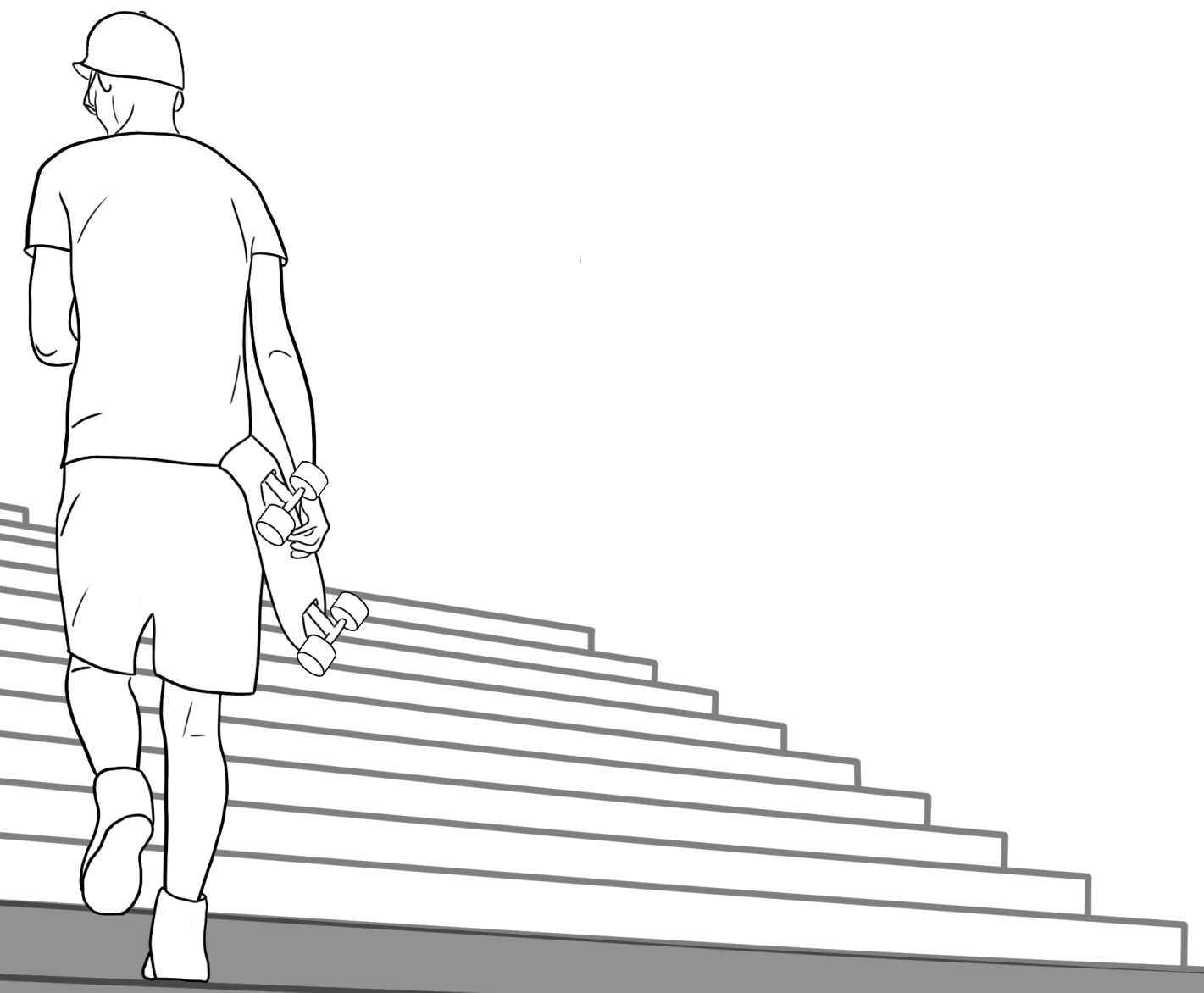




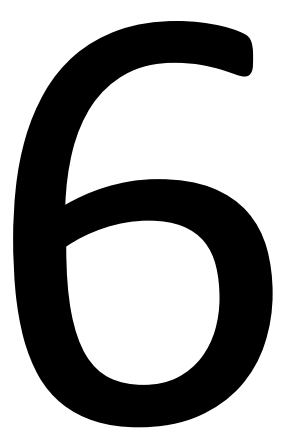

\section{Health brokers: how can they help deal with the wickedness of public health problems?}

Celeste E van Rinsum, Sanne MPL Gerards, Geert M Rutten, len AM van de Goor, Stef PJ Kremers

BioMed Research International, 2017; 2017: 1-10 


\section{ABSTRACT}

Background: The role of health broker is a relatively new one in public health. Health brokers aim to create support for efforts to optimise health promotion in complex or even 'wicked' public health contexts by facilitating intersectoral collaborations and by exchanging knowledge with different stakeholders. The current study aimed to explore the role of health brokers, by examining the motivational, contextual, and behaviour-related factors they have to deal with.

Methods: Fifteen professionals from various backgrounds and from various policy and practice organisations were recruited for a semistructured interview. To structure the interviews, we developed the 'Health Broker Wheel' (HBW), a framework we then specified with more details derived from the interviews.

Results: We identified seven primary types of behaviour that health brokers need to engage in: recognizing opportunities, agenda setting, implementing, network formation, intersectoral collaboration, adaptivemanaging, and leadership.Determinants of health brokers' behaviours were identified and categorised as capability, opportunities, motivation, and local or national contextual factors.

Conclusion: The health brokers' role can be seen as an operational approach and is visualised in the HBW. This framework can assist further research to monitor and evaluate this role, and health promotion practitioners can use it as a tool to implement the health brokers' role and to facilitate intersectoral collaboration. 


\section{BACKGROUND}

Many previous studies have reported on the complexity of emerging worldwide public health problems, such as overweight and obesity [1, 13, 182-184]. The causes of overweight and obesity are complex and there are many underlying interactions between the determinants $[8,79,185]$. It has proved to be difficult to address these causes with interventions, due to this complexity and the variety of determinants [15].

Addressing such complex or even 'wicked' health problems requires a combination of solutions, involving different sectors, such as business, industry, education, spatial planning, public health care, welfare, sports, housing, civil affairs, agriculture, transportation, public safety, and media [3, 186-192]. An integrated or intersectoral approach is often regarded as the optimal way to prevent complex public health problems, such as obesity and socioeconomic health disparities [17, 187, 193, 194].

Previous research has shown the benefits of facilitators, change agents, or 'catalysts' of change in connecting stakeholders and subsequently stimulating the integrated approach [76, 195-197]. In Netherlands, the role of 'health broker' was introduced a few years ago, and several municipalities have now appointed them [76]. Health brokers are social entrepreneurs [198], who can be characterised as change agents [76]. They aim to create support and establish permanent collaborations and encourage knowledge exchange among politicians, policy-makers, private parties, health promotion practitioners and citizens to improve the health of the community, to reduce the number of disadvantaged persons and to optimise evidence coproduction in the prevention of complex public health problems $[76,187,199]$. Health brokers are assumed to operate as 'anchoring points' by connecting community problems to policies and services [76, 77]. For instance, they are expected to support obesity prevention by connecting different parties at the local level, such as various municipal government sectors [75]. As such health brokers can facilitate intersectoral collaboration, combine knowledge from different stakeholders and sectors, and actively incorporate evidence into public health policy and practice.

Harting et al. [76] showed that the complexity of health issues and the local situation often impedes the health brokers' role. To date, however, little is known about the factors influencing health brokerage, such as the motivational, contextual, and competence-related factors they have to deal with. In order to be able to examine such factors, it needs to be clear which are the primary behaviours related to the implementation of the health broker role. The present study aimed to explore the role of the health brokers regarding emerging wicked health problems, by examining these primary behaviours and their determinants. We conducted a qualitative study with semistructured individual interviews with various professionals, based on a broad theoretical framework as outlined below. 


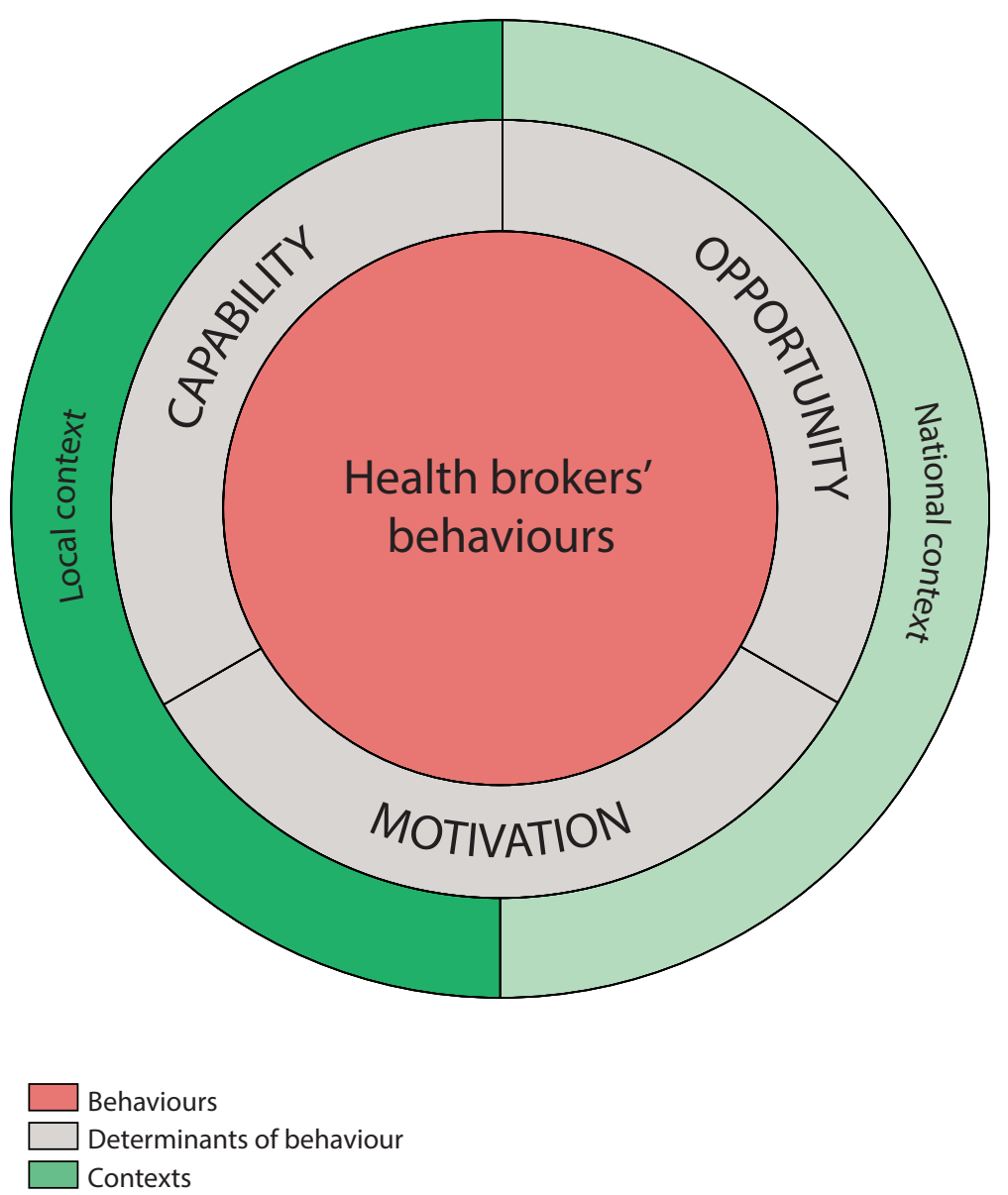

Figure 1: The core of the theoretical framework: the 'Health Broker Wheel', based on Hendriks et al. [179] and Michie et al. [200]

\section{THEORETICAL FRAMEWORK}

We based our theoretical framework, the 'Health Broker Wheel' [HBW] (see Figure 1), on the 'Behaviour Change Ball' [BCB] [179], a tool to analyse barriers and facilitators of integrated health policies within local governments [179]. The 'Behaviour Change Ball' is in its turn based on the 'Behaviour Change Wheel' [200]. In essence, we view behaviours of health brokers as determined by sociopsychological processes that underlie human motivation. This is completely in line with the 'COM-B' assumptions in the BCW: capability, opportunity, and motivation (COM) and behaviour (B) [200]. The COM-B system recognizes that behaviour change does not occur in a vacuum but will occur only when COM determinants for health brokerage are sufficiently present [179]. These determinants underlie the implementation of the behaviours [179].

Capability refers to what individuals, in this case health brokers, know or are able to do. For example, the ability to guide the process of intersectoral collaboration, to adapt to 
change and to know about integrated health policies [76, 179, 197, 200, 201]. Opportunity comprises structural variables, including all aspects of the physical and social environment that influence behaviour either directly or through motivation (e.g. through organisational cultures or organisational structures) [179]. Motivation can involve automatic processes (e.g. beliefs, emotions, and work routines) [202] or more reflective conscious decision-making, such as choices that are made based on evaluations of past experiences [200]. The COM-B system is in turn influenced by different contexts and external influences, including characteristics of both the national and local contexts.

\section{METHODS}

\section{Study design and sample}

This qualitative study involved semistructured interviews, held between April and June 2013, in which fifteen health promotion professionals from across Netherlands were invited to participate. These professionals had different perspectives on the field of health brokerage. Since the goal of the study was explorative and pioneering,we wanted to interview a wide range of professionals in order to get a relatively broad perspective on relevant behaviours and beliefs, rather than to aim for saturation and deeper information in a small range of interviewees. All professionals were recruited using a snowball method [203]. The inclusion criteria were as follows: having knowledge about the topic of health brokers, based on experiences with or as a health broker, and working in health promotion. The participants were sent an e-mail explaining the topic and goal of the interview. A few days after they had received the e-mail, they were contacted by telephone or e-mail to further explain the study procedure and to set a date for the interview. All of the professionals who were invited agreed to participate in the present study. They were included after they had given permission to record their interview.

The study sample consisted of two health brokers, three former health brokers, two (senior) health policy advisors from the public health services [PHS], one health promotion professional from the PHS, one project leader from the PHS, one former project leader from the National Institute for Health Promotion and Disease Prevention, two PHS managers, two researchers from different universities, and one self-employed public health consultant. This made a total of fifteen interviewees, of which three were men. The health brokers and other professionals were working in a heterogeneous set of municipalities, geographically spread throughout Netherlands. The health brokers were generally structurally embedded in the public health department of their municipality or at the PHS.

\section{Interview procedure}

The interviews were held by the first author at locations chosen by the interviewees. The interview structure (see Appendix) was based on the HBW. Examples of the questions are as follows: "What factors influence a health broker's work?" and "Can you describe the responsibilities of health brokers in your region?" The health brokers were 
asked additional questions concerning how much they enjoyed their work. The interviews were estimated to take one hour. The core of the HBW (see Figure 1) was developed and applied in this study by starting with the central COM-B system and we filled in the different factors per level (i.e. behaviours, determinants, and contexts) based on the interviews $[76,179]$.

\section{Data analysis}

The recorded interviews were transcribed verbatim. These transcripts were coded based on the core of the HBW, using NVivo 11.0 software. New thematic codes were made driven by the data. Three interviews were coded by two researchers (CvR and SG). Discrepancies between the two coders were discussed with a third researcher (SK) until agreement had been reached, after which the first author coded the remaining interviews. After the first analyses, the results were summarised for each participant and sent to them for a member check [204]. Only one interviewee made some textual additions.

\section{RESULTS}

\section{Behaviours}

Using the interviews data we compiled the inner level (i.e. health brokers' behaviour; see Figure 2). Overall, the interviewees stated that the main task of health brokers is to facilitate intersectoral collaboration to improve public health (see Table 1 for an overview of the different health brokers' behaviours and Table 2 for a quotation per each HBW component). They emphasised that intersectoral collaboration is a prerequisite for implementing changes in the physical and social environment, the system, and the policies within a health broker's district. One health broker mentioned that small changes in nonhealth sectors can have a significant impact on health. Topics the health brokers worked on included socioeconomic health disparities, lifestyle themes (e.g. overweight and alcohol consumption), the physical environment (e.g. indoor environment), and loneliness among the elderly.

According to the interviewees, health brokers have to create support and encourage stakeholders to get involved. Participants indicated that many stakeholders do not realise that they can play a part in intersectoral collaboration for promoting health. This is because they do not know exactly how they can play a role in 'health', as that is not their core business. In addition, people work in areas closely related to health and may have different terminologies when talking about health. For example, the Department of Spatial Planning may build more cycle lanes, thereby influencing people's health, but they may not talk about it in terms of health. In order to facilitate agenda setting for health in different sectors, the interviewees proposed that health brokers should use more appealing and positive terms, instead of 'health' or 'prevention'. Furthermore, the health brokers emphasised that the benefits for nonhealth sectors need to be visible for these actors, if they are to participate in intersectoral collaboration. 


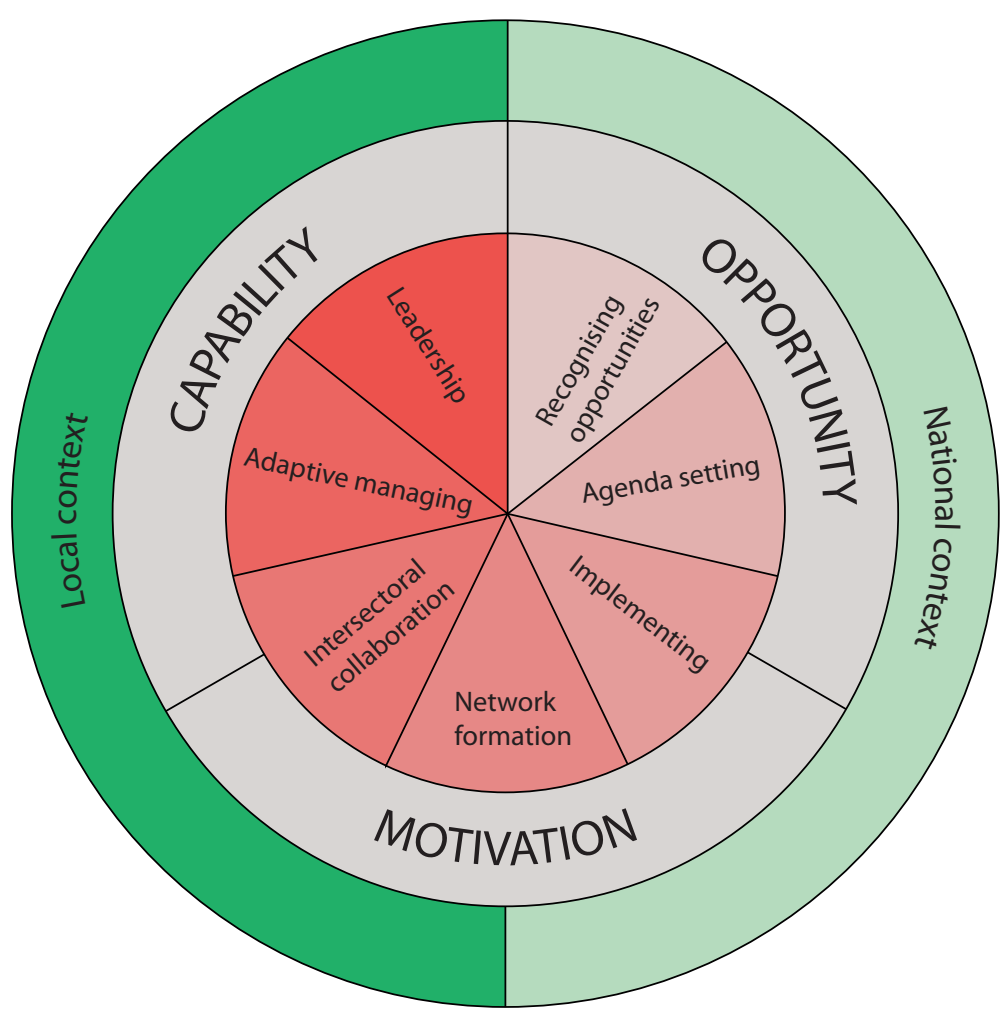

Behaviours

Determinants of behaviour

Contexts

Figure 2: The final version of the theoretical framework: the 'Health Broker Wheel'

The interviewees explained that health brokers have a reinforcing task within the network. The core of their binding role is collaborating with practice (e.g. primary care institutions) and policy and with the public health sector. Their job involves identifying the most important health problems (recognizing opportunities) and putting them on the political decision-making agenda (agenda setting). Another important task that was mentioned is that of initiating projects or using existing products (implementing). Likewise, health brokers can look for new channels in their district to reach the target group and engage in discussions about health with citizens (network formation). Further important health broker behaviours mentioned by the interviewees were lobbying, completing projects, binding parties, creating support, collaborating, empowering people, adapting to the local context (adaptive management), understanding and speaking the language of the different stakeholders, empathising with others, and focusing on how to make prevention or preventive projects sustainable (leadership), such as advising to make policies. Interviewees also mentioned that if there had been no health brokers, things might have evolved more slowly, because stakeholders would be more likely to stay within their own sector and not collaborate. 
Table 1: Behavioural components of the 'Health BrokerWheel'

\begin{tabular}{|c|c|}
\hline Behaviours & Components \\
\hline Recognising opportunities & $\begin{array}{l}\text { - Identifying the most important health problems; and being } \\
\text { aware that citizens can perceive other problems than the statis- } \\
\text { tics indicate } \\
\text { - Seeing where opportunities and chances are } \\
\text { - Pioneering }\end{array}$ \\
\hline Agenda setting & $\begin{array}{l}\text { - Lobbying } \\
\text { - Showing the benefits for non-health sectors } \\
\text { - Get others to contribute to health } \\
\text { - Putting health on the political decision-making agenda }\end{array}$ \\
\hline Implementing & - Initiating and completing projects \\
\hline Network formation & $\begin{array}{l}\text { - } \quad \text { Binding parties } \\
\text { - } \quad \text { Creating support }\end{array}$ \\
\hline Intersectoral collaboration & $\begin{array}{l}\text { - } \text { Collaborating } \\
\text { - Teamwork } \\
\text { - Mediating } \\
\text { - Empowering } \\
\text { - Discussing }\end{array}$ \\
\hline Adaptive management & $\begin{array}{l}\text { - } \text { Adjusting to the local context } \\
\text { - Speaking the language of different stakeholders } \\
\text { - Empathising with others }\end{array}$ \\
\hline Leadership & $\begin{array}{l}\text { - Having a vision for the future } \\
\text { - Focusing on sustainable cooperation }\end{array}$ \\
\hline
\end{tabular}

\section{Determinants of behaviour}

\section{Capability}

Health brokers' competences mentioned by the interviewees include being assertive, being flexible, being patient, not being afraid to be corrected, and knowing how municipal governments work. The respondents stated that it is not sufficient for health brokers to only know about the available health interventions and their scientific foundation but that they should also have knowledge regarding health promotion. However, a background in health promotion was not required, as health brokers do not implement health promotion interventions (at the operational level), but they facilitate these implementations. A background in social science was said to be more useful, because health brokers from outside the health sector can operate more independently. Finally, the complexity of the job, including communication with and switching between (the strategic, tactical, and operational) policy levels, requires highly communicative people with academic skills.

\section{Opportunities}

The interviewees reported that successful intersectoral collaboration required resources to be available, such as information, space, time (for stakeholders to collaborate), 
Table 2: Quotations to illustrate the components of the 'Health Broker Wheel'

\begin{tabular}{|c|c|}
\hline Components & $\begin{array}{l}\text { Quotations regarding the health brokers' behaviours, their determinants } \\
\text { and the different contexts of the health brokers' work }\end{array}$ \\
\hline \multicolumn{2}{|l|}{ Behaviours } \\
\hline Recognising opportunities & $\begin{array}{l}\text { "A health broker in The Hague consistently said: 'I'm not going to commit } \\
\text { myself to a particular activity or theme or whatever. I'm going to look what } \\
\text { is going on here, how I can help and how I can convey the residents' wishes } \\
\text { to the policy official.'... I thought that this health broker in The Hague had } \\
\text { the purest role, because she was not tied to anybody." (Former project } \\
\text { leader) }\end{array}$ \\
\hline Agenda setting & $\begin{array}{l}\text { "It is important to show that there is a benefit to be gained for the other } \\
\text { sector. If you can make that click, then you have somebody on board." } \\
\text { (Former project leader) }\end{array}$ \\
\hline Implementing & $\begin{array}{l}\text { "We have said to the professionals that if there had been no health broker, } \\
\text { things would not have changed, because things would not get started and } \\
\text { would not be sustainable." (Health Broker) }\end{array}$ \\
\hline Network formation & $\begin{array}{l}\text { "You need to have people who know really intuitively how to get others } \\
\text { involved and how to build networks, how to deal with these processes and } \\
\text { how to get citizens involved." (Manager at PHS) }\end{array}$ \\
\hline Intersectoral collaboration & $\begin{array}{l}\text { "We particularly try to motivate the officials to engage in conversations } \\
\text { with other departments and we give them tools to do so." (Senior policy } \\
\text { functionary) }\end{array}$ \\
\hline Adaptive management & $\begin{array}{l}\text { "I think that you always need to connect with the culture, circumstances } \\
\text { and opportunities of a particular setting." (Health Broker) }\end{array}$ \\
\hline Leadership & $\begin{array}{l}\text { "I think it is very important that health brokers have the focus on making } \\
\text { things sustainable. How do we, if we initiated a couple of things, get people } \\
\text { to take autonomy and carry on with it themselves?" (Manager at PHS) }\end{array}$ \\
\hline \multicolumn{2}{|l|}{ COM } \\
\hline Capability & $\begin{array}{l}\text { "Someone who understands what residents' say in their local dialect and } \\
\text { who can explain this to the relevant officials." (Former project leader) }\end{array}$ \\
\hline Opportunity & $\begin{array}{l}\text { "There has to be willingness on the part of different parties to collaborate. } \\
\text { Otherwise, it's like flogging a dead horse. And there has to be willingness to } \\
\text { share data." (Public health consultant) }\end{array}$ \\
\hline Motivation & $\begin{array}{l}\text { "I liked to initiate the discussion and let people think about 'What could I } \\
\text { do' and 'What can I contribute to health'." (Former health broker) }\end{array}$ \\
\hline \multicolumn{2}{|l|}{ Context } \\
\hline National context & $\begin{array}{l}\text { "You can say that major structural barriers are the elections, the fact that } \\
\text { health is currently not an issue in the coalition, the fact that the left-wing } \\
\text { green party is no longer part of the coalition... Those are stumbling blocks." } \\
\text { (Former health broker) }\end{array}$ \\
\hline Local context & $\begin{array}{l}\text { "What makes it more difficult is that no two aldermen are the same, and } \\
\text { they stay only for four years. If you want to eliminate the socioeconomic } \\
\text { health disparities, you need to work in a much longer term. And yes, it is } \\
\text { supported by the alderman and the municipal executive. But speeding it up } \\
\text { and to keeping it high on the political agendas of all aldermen, that is quite } \\
\text { difficult." (Health broker) }\end{array}$ \\
\hline
\end{tabular}

Note: COM is an abbreviation of capability, opportunity and motivation. 
and funding to implement initiatives. Two respondents claimed that policy plans canmake intersectoral collaboration sustainable; nevertheless, the different parties involved must also implement these agreements.

Intersectoral collaboration was said to depend on support from the stakeholders in the community. For example, if one alderman or municipal official leaves the organisation, initiatives can collapse and have to be rebuilt. Furthermore, municipal governments are usually hierarchical, and the internal management is not always well-coordinated. Organisational compartmentalisation (an organisation without internal cross-connections and collaboration) and bureaucracy can hamper intersectoral collaboration.

\section{Motivation}

We asked participating health brokers about their motivation towards their work. They all indicated they had a positive attitude towards their role. They liked the collaboration (intersectoral or otherwise), networking, and communication (although communication was also reported to be hard). In their opinion, a health broker should get his/her work enjoyment out of the satisfaction of bringing people together, acquiring new contacts, developing new initiatives, and learning from other people's views.

Negative aspects of their work were, however, also mentioned. Health brokers said that they needed to be patient and sometimes had to let other stakeholders take the lead, which most of them did not like. Another perceived disadvantage was their dependence on other people. Although the health brokers were highly motivated to reduce health problems, their work was not very concrete or visible, which made it hard to keep focusing on the main goal and to enjoy their 'successes'. The difficulty of reporting results, such as the fact that results are mostly visible in nonhealth sectors and on the long term, and being accountable for their actions were also mentioned as negative aspects of their work. Furthermore, they mentioned that the work is very wide-ranging and many different stakeholders are involved, which sometimes makes it difficult to retain the overall picture.

\section{Contexts}

With the exception of one interviewee, everyone agreed that being a health broker is not so much a job but should be seen as a work attitude, a role, or a working approach. It requires flexibility towards setting goals and taking a demand-driven approach to work. According to the interviewees, a health broker should work and switch between the strategic, tactical, and operational policy levels. Health brokers who switch between the different levels can achieve more than those who work at one of the three levels only. Therefore, the ideal option would be to spread the workload and the three different policy levels over two or three health brokers at all three policy levels in each region.

The interviewees believed the local context was an important factor influencing the determinants of health brokers' behaviours. For example, the differences between urban and rural municipalities were considered to be relevant external factors, especially since health problems are often more prevalent and clustered in deprived areas in 
cities. Interviewees emphasised that in cities there are already many partnerships in place between organisations, which makes intersectoral collaboration easier. On the other hand, there is often more social cohesion in rural areas than in urban areas, which increases the chances of successfully implementing integrated approaches to prevent obesity.

Another aspect of the local context concerns the responsibilities of officials in urban and rural municipal governments. According to the respondents, municipal officials within cities are often responsible only for the public health sector, whereas their counterparts in smaller municipalities have multiple responsibilities in addition to public health. As a consequence, health is typically one of their lowest priorities and less financial resources are available. However, there were also other impediments for health brokers in larger cities.The respondents noted that since urban municipalities have larger numbers of employees, the chances of 'serendipity' (beneficial coincidences) are smaller. Therefore, as one interviewee argued, simply having people physically further removed from their colleagues means they will have fewer conversations with them.

The interviewees stated that the governmental cutbacks within the national context, which started in 2012, led to national decentralisation, enhanced the responsibilities of municipalities for the health of their citizens [205], and affected municipal budgets, health policy budgets, and intersectoral collaboration. The interviewees believed that the perception of municipal authorities is that prevention of obesity will require a lot of national financial support. However, respondents stated that financial resources can have both a beneficial and an inhibiting impact on the health brokers' work. On the one hand, lack of funding can lead to intersectoral dependence, resulting in cooperation and combining the financial resources. On the other hand, a lack of resources can increase territoriality, which means that people stay within their own sector or area. The lack of resources implies that organisations or sectors (also at the local level) need to be selective in projects or initiatives, which can mean that a health project becomes the last priority. Interviewees mentioned that a 'working budget' for health brokers may facilitate the initiation of local activities. This overcomes barriers of territoriality and competent (i.e. highly 'capable') health brokers increase the stakeholders' perceived opportunities to collaborate on the one hand and the motivation to do so on the other.

In addition to financial issues, decentralisation of health care responsibilities, government elections, and the subsequent process of building political coalitions are also influential factors at national level. Furthermore, a former project leader and a manager stated that public-private collaboration is important in integrated efforts to prevent obesity and that this collaboration needs to be strengthened. Public-private collaboration means that private companies, such as health insurers, collaborate with public sector or semipublic sector organisations, such as the PHS. Health brokers can also have a bridge-building or boundary-spanning function in this respect. 


\section{DISCUSSION}

The objective of the current qualitative study was to explore the role of health brokers regarding emerging 'wicked' public health problems, by examining the primary health brokerage behaviours and the various types of determinants and contexts that influence them. We developed the 'Health Broker Wheel' [HBW], which provides a framework for these behaviours and their determinants. Our insights are based on perceptions of closely involved professionals and a survey of the relevant literature. The present study identified that health brokers need to engage in seven different types of behaviour to fulfil their role and that they need to possess certain competences to address wicked health problems. It should be noted that all HBW components interact with each other, both within and between the levels. The levels can rotate relative to each other, which is why the components are presented in the form of a wheel $[179,200]$.

The inner part of the HBW shows these seven types of health brokers' behaviours. Recognizing opportunities means scouting locally for the most urgent health problems, as indicated by both citizens and statistics, and identifying opportunities to tackle a problem. Agenda setting is the first stage toprioritize the health problems and to make stakeholders not involved in health care aware of the health problem [179]. Such nonhealth sectors mostly do not realise that they can play a role in promoting health. Hence, health brokers try to get them involved and lobby to put health on the political decision-making agenda. This can be done by reframing the health terminology in such a way that stakeholders understand how they can contribute to the intersectoral collaboration $[197,206]$. When health problems are being recognized as important to address, health brokers can implement projects to promote health by initiating them, but also by completing them. As an integral part of their work, the health brokers create networks by seeking support among stakeholders, encouraging them to get involved, forming a group of various stakeholders and getting these different parties to collaborate across sectors. Health brokers typically guide this process of intersectoral collaboration, that is, mediating between and empowering the stakeholders, so that the different parties collaborate and can work as a team [207]. Adaptive managing means that health brokers adapt their behaviour to the local context and understand and speak the language of the different stakeholders. Important components of this behaviour are empathising with the stakeholders and having an open and learning attitude [179]. Collaborations need to be managed through good leadership to formulate a clear vision for addressing health problems [208], to maintain a comprehensive view of all projects and to make sure that prevention or preventive actions will become sustainable in terms of integrated policies.

The most important behaviour expected of health brokers consists of facilitating intersectoral collaboration, for example, between policy and practice in order to improve public health. Previous research has found that intersectoral collaboration and building support are essential behaviours for people in similar positions, such as public managers [76, 209]. In addition to facilitating intersectoral collaboration, the goal of the health brokers' work is to facilitate changes in nonhealth sectors and to make these changes sustainable by means of changes at the policy level. This is comparable to the work of 
a policy entrepreneur, who links different parties in order to shape policies [210]. Other studies have also identified this strategic, big-picture thinking [209], and long-term perspective [197] as important prerequisites for maintaining intersectoral collaboration [211]. Long-term policies would be most effective in terms of achieving intersectoral collaboration when they are imposed by the national or local government [212]. Longterm policies can also lead to changes in nonhealth sectors, which is beneficial and supportive for the health brokers' role.

The health brokers' job requires multiple competences such as being flexible, keeping up with the scientific evidence base in multiple fields, and maintaining contacts with different policy levels and sectors. The competences of health brokers relate to social and interpersonal communication skills, as Koelen et al. [188] and McGuire [209] also stated. Research has demonstrated that 'boundary spanners' or similar professionals can use a personal approach to create a shared interest and build social capital and trust $[188,196,213]$. Various studies have shown that trust among stakeholders is essential for teamwork and for building sustainable relationships [77, 188, 196, 209, 214, 215]. Where collaborations already exist, trust is more likely to be building up. However, building trust takes time and occurs throughout the collaboration process [209]. As a consequence, a health broker's main interest in this respect is to initiate collaboration and subsequently ensure its continuation.

Successful intersectoral collaboration is also influenced by opportunities. The interviewees indicated that resources and support from stakeholders have a positive effect on intersectoral collaboration and hence on the health brokers' work. One of these resources is time, which is required to build trust and develop policies [188, 197, 216].

Furthermore, health brokers indicated that outcomes of their work are not always visible, which negatively influences their motivation, since improvements in health and intersectoral collaboration usually only become visible to other stakeholders in the long term and within nonhealth sectors. Hendriks et al. [206] also addressed problems associated with making outcomes visible in the short term. However, since visibility increases the intrinsic motivation of stakeholders (e.g. funding agencies) to collaborate [188], it is important for health brokers tomake short-termsuccesses (on intermediate outcomes) visible and to publicise them.

The national and local contexts were perceived to have a strong influence on the health brokers' work. Factors at the national level include decentralisation [205], elections, cutbacks, financial support from the government, policies, health care, developments in society, and public-private partnerships. Local-level characteristics that were mentioned included the size of the community, organisational characteristics, social networks, financial resources, public-private partnerships, and current and past collaborations, which impact on the social cohesion in the region (the degree of mutual trust), as was also found in earlier research [209, 211]. As Hendriks et al. [206] stated, municipal managers should be responsible for multiple sectors to facilitate intersectoral collaboration within the organisation. At the same time, Steenbakkers et al. [197] argued that managers should collaborate more with other sectors. The degree to which municipal 
authorities work in an integrated way may be more important than the differences between urban and rural contexts. Health brokers need to take these factors into account in the different contexts when designing implementation plans for their work.

Collaboration is also influenced by the positioning of health brokers. Health brokers need to switch between the three different policy levels, so it is preferable to have multiple health brokers at all three policy levels within one region, as was also suggested by Harting et al. [76]. Since health brokers' positioning depends on the existing contexts, these contexts must be assessed before positioning health brokers within a region.

\section{Strengths and limitations}

A strength of this study was the use of a theoretical framework to structure the interviews. A limitation was that we did not interview aldermen or local public health policy officers, so the perspective of the municipal authorities is missing. In addition, on average only two professionals were interviewed for each type of job, which does not ensure data saturation. However, since our sample included a variety of professionals working in the policy and practice of Dutch health promotion and who were familiar with the functions of health brokers, we expect that our results provide a well-substantiated view of the health brokers' job. Based on the explorative nature of this study, we opted for interviewing a relatively wide range of professionals to get a broad perspective, rather than to aim for data saturation and more in-depth information from a narrow range of interviewees (e.g. by focusing on health brokers only). Member checks helped improve the reliability of our research.

\section{Recommendations}

Countries that aim to engage policymakers and practitioners in coproduction and coimplementation of health promotion activities may want to include health brokers in their public health system. Since the health brokers' COM-B system has been specified in the HBW, we recommend the use of this framework as a tool when implementing the health brokers' role in a new region. Careful consideration should be given to the question where to position the health brokers and how their positioning fits the complexity of the local context. Furthermore, our study can be seen as a first step in making the health brokers' behaviours more visible. Further studies are needed to provide more in-depth information on (sub)behaviours and their determinants. The HBW could be a useful framework for such studies or for studies addressing other coproduction activities, for example by guiding interview structures in qualitative research.

\section{CONCLUSION}

Our findings show that health brokers can make useful contributions to address 'wicked' public health problems. These problems require intersectoral collaboration and adapting to contextual factors. By operating as 'anchoring points' in connecting community problems to policies and services, health brokers represent a good example of bringing 
together evidence and health policy and practice in addressing complex public health problems. The health brokers' role can be seen as an operational approach and is visualised in the HBW. This framework can help further research tomonitor and evaluate this role, and health promotion practitioners can use it as a tool to implement the health broker role and to facilitate intersectoral collaborations.

\section{APPENDIX}

\section{Structure of interviews}

\section{Researchers/managers}

1. What is your background or relation with health brokers?

2. Can you describe the responsibilities of health brokers in your region?

3. What, according to you, is the added value/goal of the deployment of health brokers, with regard to policy, target group and organisations within the neighbourhood?

4. In your opinion how do health brokers influence health problems and intersectoral care?

5. What factors influence a health broker's work? Can you give both positive and negative examples?

6. How and at what level should health brokers be deployed?

7. How can different contexts be taken into account (urban compared to rural contexts)?

8. What successes have health brokers accomplished? Why are they successes?

9. Which problems arose in the implementation of the health brokers' role? Why did they happen?

10. What would have happened if there had been no health broker?

11. What should be improved in the future with respect to this role?

12. Can you give some advice to a PHS which has only just heard of health brokers; what should they take into account and where should they position health brokers in the organisation?

13. Can you give examples of functions comparable to that of health brokers in other sectors or countries?

14. What would be the ideal picture of a health broker? What would work best?

15. Are there any important other matters you want to mention?

16. Do you know other people I could interview?

\section{Health brokers}

1. Can you describe a normal working day? What are your responsibilities?

2. What do you like about your job?

3. What do you not like about your job?

4. What is the most important contribution your work makes?

5. In what way does your work influence the problem of overweight (or other health problems) and intersectoral collaboration?

6. What successes have you, as a health broker, accomplished? Why are they suc- 
112 Chapter 6

cesses?

7. Which problems have you experienced during your work?

8. How can you take into account different contexts (urban compared to rural contexts)?

9. How and at what level should health brokers be deployed?

10. What if there had been no health broker?

11. What should be improved in the future with respect to this role?

12. Can you give some advice to a PHS which has only just heard of health brokers; what should they take into account and where should they position health brokers in the organisation?

13. Can you give examples of functions comparable to that of a health broker in other sectors or countries?

14. What would be the ideal picture of a health broker? What would work best?

15. Are there any other important matters you want to mention?

16. Do you know other people I could interview? 


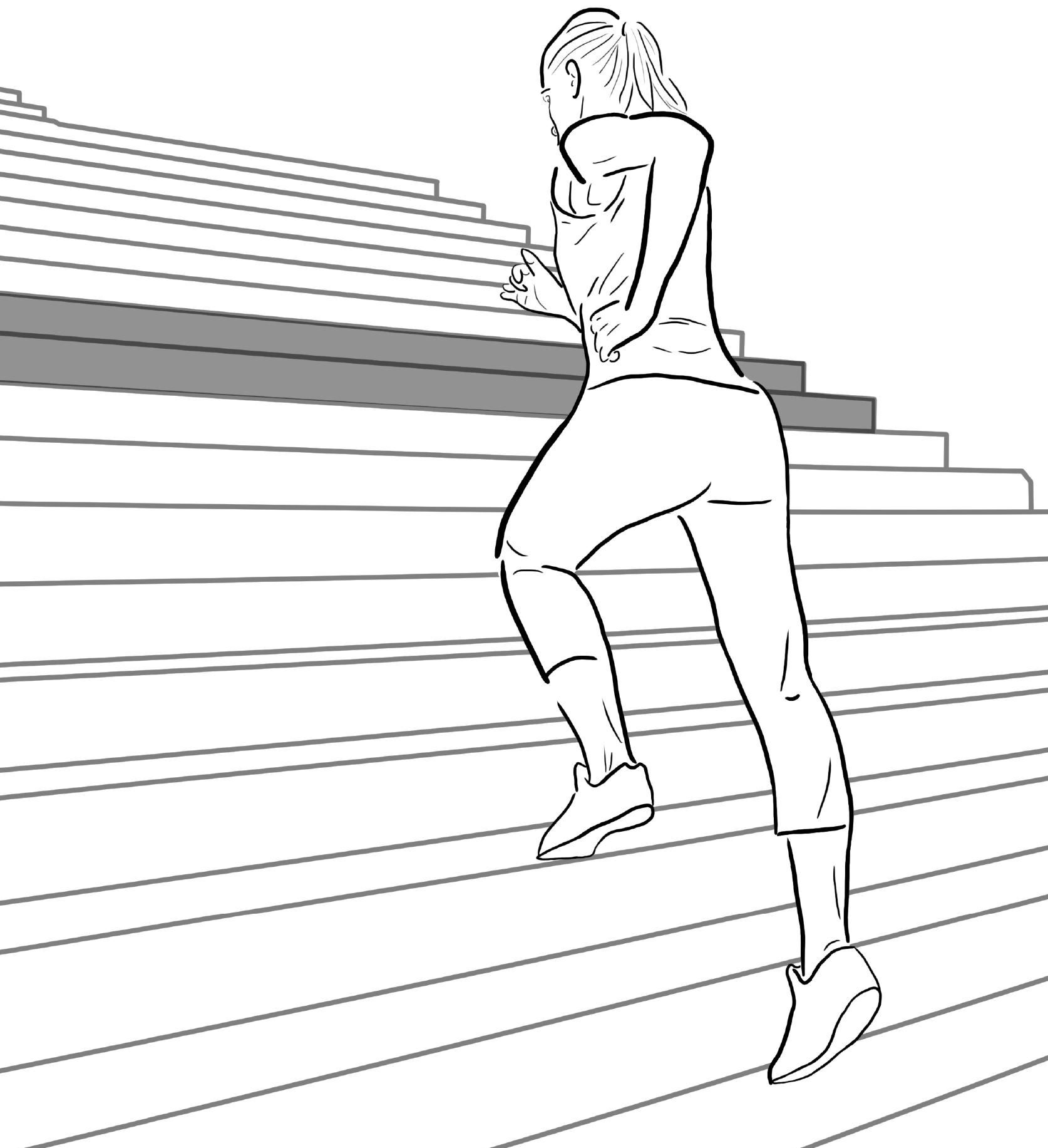




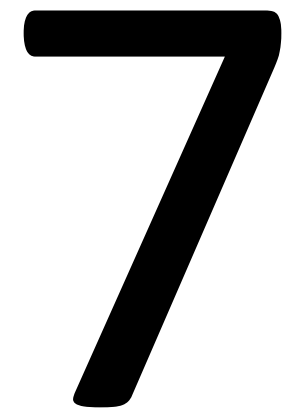

General discussion

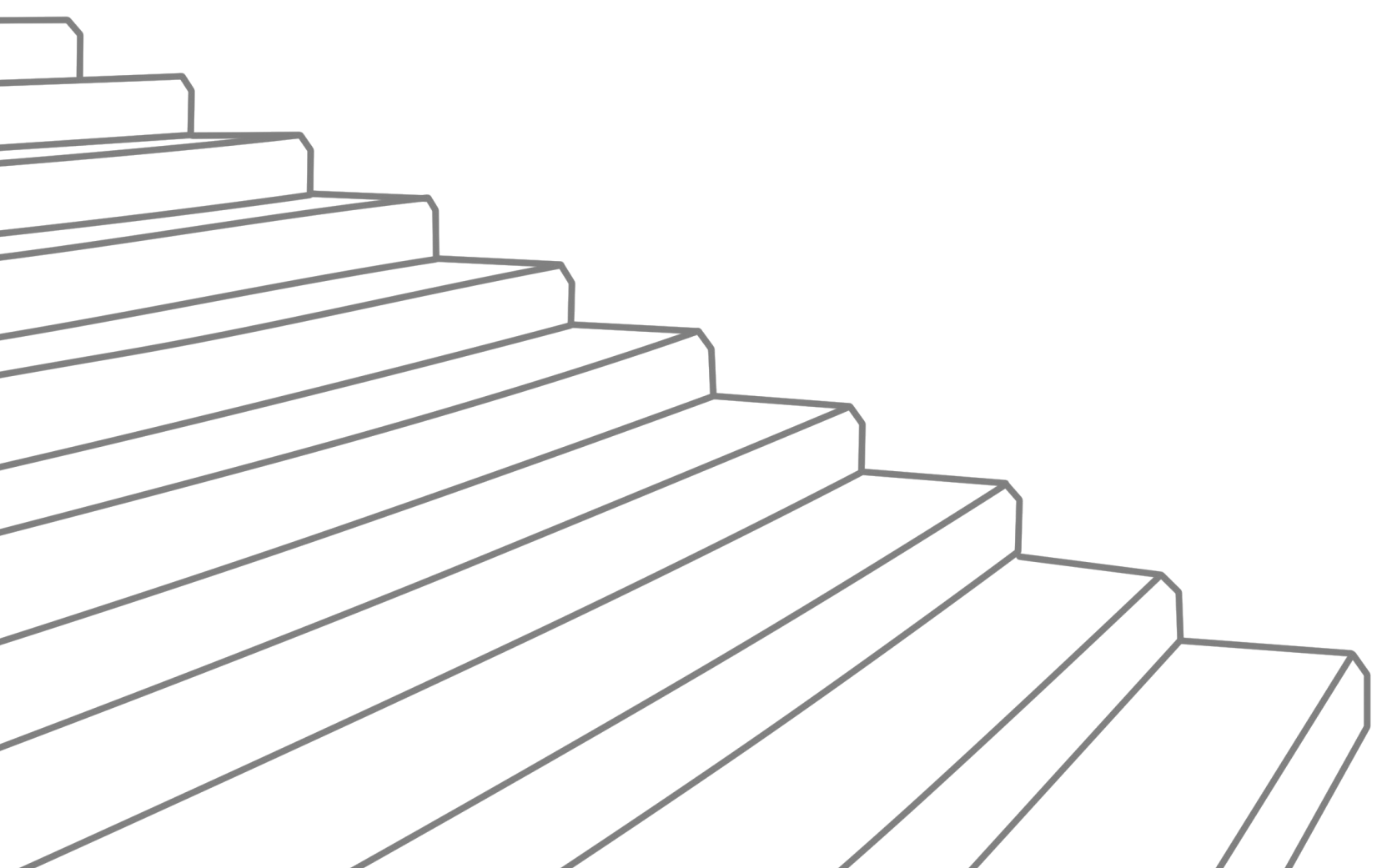





\section{PREVENTION AND TREATMENT OF OBESITY}

The prevalence of overweight and obesity is still increasing worldwide and has reached alarming numbers [2]. Over the last two decades, many preventive interventions, treatment solutions and policies have been implemented. All these initiatives have the same goal: reducing the prevalence of overweight and obesity. Due to the complexity of the obesity problem, a combination of different strategies addressing various health determinants is required to tackle it [15]. Both the environment and policies need to provide opportunities for people to live a healthy life, as well as personal guidance in changing one's lifestyle into a healthier one [17]. This has been the goal of obesity prevention and care for decades [217]. However, the changes in practice and policy needed to achieve this integrated perspective are proceeding quite slowly. Furthermore, different sectors, such as the health and non-health sectors, are poorly aligned regarding the prevention and treatment of obesity. Therefore, certain professionals can play an important role by functioning as linchpins (i.e. having a central and connecting role) in the network of obesity prevention, thereby improving the integration of care. They can connect and bring together different sectors and domains.

The studies reported on in this thesis investigated potential solutions at different prevention levels. The central research question was: Can lifestyle coaches and health brokers help to bridge the gap between the prevention and health care domains regarding overweight and obesity? The primary purpose of the studies in this thesis was to investigate the added value of professionals with a brokering role in the care system regarding obesity prevention and treatment. Two types of professionals have been studied in relation to this brokering function: lifestyle coaches and health brokers. More specifically, the studies in this thesis focused on facilitating and impeding factors concerning the implementation of the Coaching on Lifestyle ( $\mathrm{CooL}$ ) intervention and on behaviours and determinants of the health brokers' role at the community level in the Netherlands.

\section{MAIN FINDINGS}

The professional lifestyle coaches in the Dutch Coaching on Lifestyle (CooL) intervention counsel adults and children (and/or their parents) who are obese or at high risk of obesity, to help them achieve a sustained healthier lifestyle. Chapter 2 presents the study protocol of the evaluation study of the CooL intervention, including a description of the intervention and the qualitative and quantitative measurements used in the study.

Chapter 3 presents an overview of the results of the evaluation of the Cool intervention, which are elaborated in more detail in Chapters 4 and 5. Chapter 4 describes in detail the participants' lifestyle changes over time. It shows that this combined lifestyle intervention (CLI) has the potential to achieve some successes in coaching adult participants towards sustained behavioural change and health outcomes during the intervention period. Mixed results and smaller effect sizes were found for children and their parents.

Chapter 5 describes the detailed results of the process evaluation of implementing the 
CooL intervention, and the facilitating and impeding factors of this implementation. On average, the participants were satisfied with the programme and their lifestyle coaches. The coaches were found to have a higher work engagement than average Dutch employees and they perceived empathising with participants as their best competence. The CooL intervention was implemented in multiple regions, thanks to the efforts of many stakeholders. The results for adults in particular showed that lifestyle coaches can fulfil an important role as binding element in the health care chain of obesity prevention, by connecting the care sector and the public sector. However, we also found that the implementation process still needs to be strengthened in terms of creating stakeholder support and increasing their awareness of the concept of lifestyle coaches, since the lifestyle coach is a novel profession in the Dutch health care system. To boost this process, lifestyle coaches should engage more intensively in networking activities and entrepreneurship.

Chapter 6 discusses the primary behaviours and determinants of the role of health brokers, which, like that of the lifestyle coaches, is a relatively new role in public health. The findings showed that health brokers can make useful contributions in addressing complex public health problems, such as obesity. The health brokers' role was visualised in the theoretical framework called the 'Health Broker Wheel', showing the concepts of capability, opportunity, motivation and behaviour.

\section{LIFESTYLE COACHES IN THE COOL STUDY}

\section{Lifestyle coaches in their brokering role: entrepreneurship and networking}

Ideally, lifestyle coaches have a brokering role in the integrated approach to obesity prevention, as obesity needs to be tackled by addressing several behavioural areas and care domains. First of all, their role involves integrating and addressing all major behavioural areas linked to obesity and lifestyle, such as physical activity and dietary behaviours, in the CooL programme. This role as a health care provider is their primary task. Second, they collaborate with public health and care professionals from whom they receive referrals and to whom they can refer participants. They refer participants to other professionals if this is requested by the participants or if the matter is beyond their professional capacity. The lifestyle coaches in our study could possibly have referred more participants to other professionals. However, we found that they were not yet sufficiently aware of the possibilities for referring, and had not yet acquired enough skills and confidence to fulfil this brokering role. During the study period, the lifestyle coaches had to find their own place within the network, which depended on existing connections and relationships of trust. It became clear that children in complex family situations in particular should be referred earlier, especially to care that best fits the problems in their family.

Since lifestyle coaches were a new phenomenon in the region, fulfilling their linchpin role asked for entrepreneurial activities. However, these had not been included in their 
professional education. The lifestyle coaches also evaluated entrepreneurship as their least strong competence, while at the same time indicating this as the least important competence to have as a lifestyle coach. In addition, the lifestyle coaches argued that entrepreneurship was only necessary for the implementation phase, as a start-up. In the regions where the lifestyle coaches were not familiar with the network, or when there were many referrers, the coaches reported that it was difficult to have good contacts with all the stakeholders. Besides, they did not always fully use their entrepreneurial skills, because they assumed that actively contacting referrers was not allowed according to the CooL study protocol, and they invested little in sustaining these relationships.

The lifestyle coaches perceived their networking skills to be better and more important than their entrepreneurial skills. In fact, however, their networking competence scored below average compared to other competences. It has been shown that networking is necessary in all the implementation phases of interventions [168]. Another study found that general practitioners referred more patients to dieticians if they had frequent contacts with these professionals [218]. Since referrers' interest in a programme can decrease over time, it is good to have regular personal contacts with them to inform them about the progress of the programme participants. Furthermore, if the professional carrying out the intervention has a more central position in a dense network, the implementation is expected to be more successful, relationships are better, more trust is built up and intervention effect sizes increase $[173,219,220]$.

Research has demonstrated that brokering professionals can use a personal approach to create a shared interest and build social capital and trust $[188,196]$. Various studies have shown that trust among stakeholders is essential for teamwork and for building sustainable relationships $[77,188,196,215]$. Where collaborations already exist, trust is more likely to be built up. However, building trust takes time and occurs throughout the collaboration process [209]. Hence, lifestyle coaches need to be trained and encouraged specifically in entrepreneurship, networking, signalling and referring to other health care professionals and key persons in the public domain.

\section{Assistance by health brokers and other professionals in the lifestyle coaches' brokering role}

Lifestyle coaches operate in the health care domain, and their primary role is being a care provider, which may hamper their opportunities to address other domains. 'Ambassadors' or other 'brokers' can assist lifestyle coaches with their networking activities. An 'ambassador' should be in close contact with the stakeholders in the region. If this person is not the lifestyle coach, the role could be fulfilled by a local project leader, someone from a central organisation (e.g. health care group, which coordinate chronic care) or a person holding a central position in the network of public health and health care (e.g. a health broker). One region that started to implement CooL after the pilot period actually employed such a broker, and the recruitment rate in this particular region was much higher than in the regions that participated in our pilot study, with approximately the same number of people referred within a half year as were referred in three years in the other regions. This may be attributed to the broker's networking role and 
the more intensive contact with referrers.

The terms 'brokers' and 'bridges' are not new in the health care sector, because of the many isolated clusters (e.g. so-called professional 'tribes' and clinical versus managerial domains) [173]. Examples of such brokers between the primary care sector and the physical activity professionals in the public domain in the Netherlands are the so-called care-sport connectors (e.g. neighbourhood sports coaches) [221]. These brokers could assist the lifestyle coaches in getting to know other network parties.

Lifestyle coaches could benefit from the help of health brokers regarding networking, since the health brokers' core role is binding parties and facilitating intersectoral collaboration by creating support. These brokers know the professionals in their region and they cooperate with different sectors, such as public health care, education and sports. This makes it valuable for the lifestyle coaches to collaborate with such health brokers to get a better picture of the 'social map' of services and opportunities in the public domain and professionals in the network. Besides, health brokers aim to build sustainable relationships, and it is useful for lifestyle coaches to collaborate with them or with other brokers and to make use of their sustainable contacts, if they cannot invest time in this themselves or if they do not feel comfortable about doing so. Nevertheless, it is a necessary prerequisite for lifestyle coaches to invest in relationships with the referrers and not only focus on coaching the participants.

\section{WHAT HAVE WE LEARNED FROM THE IMPLEMENTATION OF}

\section{THE COOL INTERVENTION?}

\section{More implementation time needed}

The study on the CooL intervention revealed the facilitating and impeding factors of a CLI in a real-world setting. We have learned what is required for their optimal implementation in practice. This can take up to a few years (see also [175]). First of all, stakeholders at all levels should be involved at an early stage to secure their support. For example, referrers should get the opportunity to experience positive effects when referring their patients. Once they support the intervention, they will be more motivated to take part in the programme and to refer patients to CooL. Second, it takes time to organise the logistics of the intervention, such as the location, for each region. Third, more time is needed for the execution phase, including informing referrers, feeding back the positive results of the intervention to the referrer, and acquiring the linchpin position.

\section{Complex network}

Support from the network is crucial for an effective implementation of a CLI, such as the CooL intervention. Over the course of the pilot period, support from the majority of the referrers was relatively low. There was a small group of very enthusiastic referrers, but the rest did not refer any patients or referred only a few. Besides, their awareness 
of the intervention decreased over time, which suggests that referrers should be more frequently reminded of the intervention. Moreover, the network, which was still under development, had a low density, whereas previous research showed that in more dense networks information spreads more efficiently to all stakeholders [173]. Therefore, more effort should be invested in creating continuous attention and support, especially as long as the network is fragmented and has a low density.

The CooL intervention was implemented in seven regions, each with their own individual network, including many different stakeholders, which made the context complex. The characteristics differed in each context, including the level of support from organisations (e.g. the local health care group or public health services), stakeholders, referrers and target groups. Adapting interventions to the complex context of the local conditions can improve its effectiveness [155, 222]. The CooL programme has been adapted to regional conditions. For example, each region organised the sports facility services in a different way. This was first mapped by the lifestyle coaches, after which they discussed the possibilities with the professionals concerned on how they could be given a role in the programme.

\section{Recruitment and inclusion strategy}

The inclusion criteria for the CooL programme should not be made too strict and should be used in a flexible way, especially for children [160]. The study showed that the strict inclusion criteria were the main reason why it appeared to be difficult to recruit enough children. The numbers of children included were smaller than estimated beforehand, as a result of their not meeting one of the inclusion criteria: having a weight-related health risk, sufficient motivation to participate and being insured with one of the participating health insurance companies. Besides, it has been suggested that the youth health care (YHC) nurses in particular lacked the knowledge and skills to discuss children's overweight with their parents [223]. This could have influenced the number of referrals.

As regards the recruitment strategy, we can also learn from other similar interventions how to increase the numbers of referrals. For example, the EuroFIT intervention has been relatively successful in terms of recruitment [224, 225]. EuroFIT is an intervention to support male football fans to become more physically active and adopt better dietary behaviours, by having them exercise at the stadium of their favourite club. This concept appears to result in an increased feeling of solidarity and togetherness. Another study showed that such feelings encouraged people to participate, which boosted the recruitment process [226]. When interventions reach more people, their impact will be larger.

Another recruitment strategy could focus on different subgroups of the target population, such as non-Dutch speaking persons and people with a limited health literacy, and the CooL programme could be tailored to their needs. This could be done by developing a specific programme using simpler language and pictures, or securing the participation of professionals who speak their language. 


\section{Characteristics of the CooL programme}

The post-test revealed that the programme met the needs of the adults and parents, whereas the children were less satisfied about it. The qualitative data showed that the majority of the interviewees appreciated the coaching and the programme as a whole. Regarding the guidance, the CooL participants indicated in the interviews that they wanted more guidance after the basic programme.

As for the children's programme, many parents expressed the need for a more personalised care programme, so the option of attending an individual programme, or having fewer group sessions and more individual sessions, may be beneficial. If the children's programme was more tailored, this would contribute to the effectiveness of the intervention, as it would fit the needs better $[160,170]$. Children and their parents together can work more effectively on their specific goals at a personalised level, making them more responsible for their own behaviour. An advantage of the children's programme was the inclusion of home visits. People feel safer in their home environment, which provides the opportunity to build a relationship of trust with the lifestyle coach [227].

Other CLIs for children have focused more on the 'fun factor' than CooL did. Creating fun is especially important for children, but it is also relevant for adults, in order to intrinsically motivate them to change their lifestyle. Some good and inspiring examples already exist. For example, the MEND programme focuses more on playing and on being physically active [52]. It also uses attractively designed materials, making the intervention more fun. Another $\mathrm{CLI}$ for children, $\mathrm{COACH}$, has been successful in creating opportunities to start the intervention for children even in the waiting room, by trying to stimulate their physical activity [228]. In the CooL intervention, participants do not actually exercise together, but more attention can be given to motivating participants to participate and showing them how to make lifestyle change and exercise a fun activity.

As regards the length of the programme, the adults participated in the CooL programme for an average of six months, and the children, adolescents and their parents for an average of 7.5 months. Both programmes were on average 1.5 months shorter than prescribed in the protocol, as the lifestyle coaches scheduled the sessions closer together than intended. Previous studies recommended taking a minimum of one year for the basic programme to reach beneficial effects [14]. This is comparable with the new Dutch guidelines, which state that CLIs should last at least two years, including a one-year basic programme [229]. During the pilot period, the lifestyle coaches shaped and finalised the CooL programme into a new version (see the section on 'National development of CLIs and CooL' below for more information) to meet these guidelines, which is likely to be nationally implemented from 2019 onwards. This new CooL programme combines the basic and relapse prevention programme, unless there are good reasons not to attend the relapse prevention programme.

Regarding the supplementary CooL programmes, 69 adults started the relapse prevention programme during the study period (group and individual sessions) and eight persons started the additional programme (only individual sessions), after completing the basic programme. Since the relapse prevention programme started later than intended, 
due to delays in the design and implementation of the programme, the effects of these supplementary programmes have not yet been studied. Only a few participants had reached the stage where they could take part in follow-up measurements within the study period. However, it would be interesting to measure the effects of relapse prevention efforts in the future.

\section{Competences and skills for lifestyle coaching}

Lifestyle coaching requires a combination of essential skills, namely empathising with others, autonomy-supportive coaching and knowing about health risks. Also, lifestyle coaches should be easily accessible, should show no prejudices and should possess solid skills concerning professional communication with stakeholders and referrers in the network. The combination of these skills and competences requires a great deal of training, experience and knowledge from the lifestyle coaches. Comparable CLIs have incorporated professionals with a lower educational level or less intensive training regarding coaching skills $[73,230]$. Studies have shown that these professionals lacked specific coaching competences $[73,83]$.

Training courses for lifestyle coaching should therefore be given at a high educational level, because of the complexity of the coaching skills and knowledge required. Coaching skills should also be taught throughout all courses in the training programmes for health care professionals such as dieticians, physiotherapists and general practitioners. Since many different types of professionals are potentially involved in CLIs, they should all have a high basic level of coaching skills.

\section{Weight not the primary goal}

Losing weight is a commonly used outcome measure in research and practice regarding obesity prevention. The CooL research team has had many discussions about the importance of weight as an outcome, especially when the weight change among Cool participants was analysed. Some professionals and researchers interpreted the average weight loss as a small effect, and inferred that CooL was an ineffective intervention.

Weight change is commonly seen as a clear outcome variable and useful for comparing different interventions. However, just as in earlier CLIs like the BeweegKuur [34], weight loss is not the primary goal of the CooL intervention. Lifestyle coaches encourage the participants to identify challenging but achievable action steps for each of the lifestyle behaviours, in order to set feasible and sustainable goals that will improve their longterm health. The assumption is that if the focus was primarily on losing weight, participants would focus mostly on the goal, rather than on the process of getting there [231]. Therefore, we recommend not to focus on weight loss as an outcome, but on behavioural and psychological outcomes, such as physical activity, dietary behaviours and motivation [10]. This focus on other outcomes is not only important for adults, it is also important in evaluating interventions for children, as these outcomes may improve even in the absence of BMIz change [232], for instance as an increase in muscle mass. 
Nowadays, more researchers and interventions are focusing on lifestyle changes, quality of life and health gain, in addition to weight change [14], which is considered a favourable development in the evaluation of obesity prevention and treatment programmes.

\section{HEALTH BROKERS}

The findings of our studies showed that health brokers can make useful contributions to addressing complex public health problems, such as the obesity problem. Seven primary types of behaviour were identified that health brokers need to engage in: recognising opportunities, agenda setting, implementing, network formation, intersectoral collaboration, adaptive management and leadership. Determinants of health brokers' behaviours were identified and categorised as capability, opportunities, motivation and local or national contextual factors.

Health brokers have a binding role, facilitate intersectoral collaboration and connect community problems to policies and services. This implies that they are good networkers, and their job requires skills such as creating support and maintaining contacts with different policy levels and sectors. Whereas networking is at the core of the health broker's job, the lifestyle coaches appeared to lack these skills. Health brokers can therefore probably assist lifestyle coaches with these skills and their network. Since our study of health brokers did not focused specifically on obesity, we are unable to draw definitive conclusions about their role in the obesity network. However, given the complexity of, and the multi-sectorial approach to, the obesity problem, engaging health brokers in this process is expected to be beneficial.

As regards obesity prevention, health brokers can in turn benefit from the collaboration with lifestyle coaches, because of the latter's counselling relationship with the target group. Although health brokers do have contacts with the target group to investigate their needs and health problems, they can learn a lot from lifestyle coaches concerning communication and coaching. Lifestyle coaches know more about the motivation and behaviour of obese people and about the health care system regarding obesity (e.g. problems that people can encounter). This knowledge is relevant when health brokers are in dialogue with those representing the interests of citizens, such as politicians, policy makers, social and welfare workers, and private parties. Furthermore, lifestyle coaches have more contacts with professionals in the health care sector, for example general practitioners, practice nurses and YHC professionals, concerning the care for their participants. Health brokers can thus learn more about the care for one person and the different professionals that a person has to consult. This knowledge can be useful for them to understand the complexity of problems that people have and the associated issues. 


\section{METHODOLOGICAL CONSIDERATIONS}

\section{Cool study}

The CooL study monitored the implementation process of the CooL intervention and the lifestyle changes achieved by participants over time, in a one-group pre-post design, using mixed methods. The process was studied by means of group and individual interviews, observations and document analysis (qualitative). The changes over time among participants were examined by means of questionnaires and objective parameters (quantitative).

\section{Strength and limitations}

The strengths of the CooL study include its action-oriented approach, the real-world setting in different regions, the use of several implementation process research methods and instruments, and the longitudinal design. The action-oriented approach resulted in close and effective collaboration between the lifestyle coaches and the researchers, and enabled the implementation process to be closely followed and improved when needed. We were aware of the subjective view and prejudices of the researchers which are inherent to this approach. The main researcher had good contacts with the project teams and the lifestyle coaches, resulting in relationships characterised by reciprocity and equality, which is beneficial to the research requirements [233]. However, such close involvement in the process may challenge the objectivity of the researcher who does the field work. Therefore, we stimulated an objective attitude of the researcher by means of a study design applying mixed methods, including frequent discussions with all members of the research team and the involvement of various target groups (e.g. stakeholders, lifestyle coaches and participants) to receive information from different points of view.

Implementing the intervention in the real-world setting was complex, due to contextual and organisational factors. However, the chances of achieving sustainability of the CooL intervention and its nation-wide dissemination were probably greater than if the pilot had been accompanied by a controlled trial [180]. Nevertheless, to correctly test the effectiveness of the CooL programme, it would have been necessary to include a control group. Without a control group, the results cannot be compared with care as usual. Since the primary focus of this study was on the implementation process, however, testing the effectiveness was not our main objective. The one-group pre-post design limited our ability to draw conclusions about the added value of the programme in terms of effectiveness and the causal relationship between the CooL intervention and the changes achieved by participants over time. Previous studies on CLIs that did include a control group have shown relatively stable patterns of behaviour and weight within the control group $[148,149]$.

The fact that the study was executed in the currently existing national context can be considered a strong point. The national health care policies had a favourable effect on the dissemination for the dissemination of CooL after the pilot period, because during 
the study period it became clearer that CLIs would be covered by health insurance policies in the Netherlands from 2019 onwards [167]. This was seen to have a positive influence on the motivation of the lifestyle coaches, referrers and other stakeholders in both the pilot regions and the regions that started implementation after the pilot period.

The lifestyle coaches constantly adjusted and adapted the CooL programme to the participants' needs during the study period. In the early stage of the study, the lifestyle coaches designed their own custom-made programme content. Each of them worked in their own way, but used the same themes, general principles and way of thinking. Over the study period, they gradually compared their methods and combined their best practices into a final programme format. This meant that differences between the ways the coaches executed the programme and the programme fidelity among the lifestyle coaches could not be investigated systematically, because of the continuing changes to the programme over time. This may be viewed as a limitation, but we assumed that adaptation is desirable, and promoting complete programme fidelity might even reduce its effectiveness [234].

There was also a risk of social desirability in the answers given in the questionnaires. It has been shown that people who are overweight or obese underreport their dietary intake [11], and obese adults and adolescents also underreport their weight. In addition, obese adults have been found to underreport their height [150]. To reduce this bias and to minimise the number of missing variables, objectively measured BMI values were combined in our analyses with self-reported BMI. The degree of consistency between objective and self-reported weight data for adults was very high, which offers some confidence in the internal validity of these data. However, the limitations of these data should also be mentioned. Due to logistical issues, the post-test objective weight data were not measured by the research team at the same time as the questionnaires were completed. Therefore, a wider time frame was used (several months before and after the T1, T2 or T3 assessments) to link these data to the T1, T2 or T3 data. In addition to these measured data, objective weight data were retrospectively gathered from various general practitioner information systems. This made it possible to use a combination of data collection methods to calculate the BMI for each person.

\section{Missing values and numbers of participants}

Understanding how we dealt with missing values in the analyses is important for interpreting and generalising our longitudinal quantitative results, especially since the missing values rate was rather high. Firstly, sum scores for each sub-scale were calculated for each person at each measurement moment. For each scale, we allowed a maximum of two missing values. If a subscale had more than two missing values, the sum score was regarded as missing. Second, we checked whether the missing values rate of the outcome measurements was related to the demographic variables. If this was the case, the corresponding demographic covariate was included in the model. Additionally, all data from participants who did not drop out before the first group session were included in the analyses. 
Our power analysis indicated that a sample size of 235 adult participants and 135 children was required. These intended inclusion numbers were not reached in the longitudinal study, and there was a high loss to follow-up, especially regarding the questionnaires. Not all the referred people started the intervention, nor did every participant fill in all three questionnaires. Hence, the group of participants in the longitudinal analysis was smaller than the number of people referred. Although the relatively low numbers of participants that we could follow have a negative impact on the statistical power, we found multiple significant differences in the adult population. Although a research assistant phoned people to remind them to return the questionnaires, the specific reasons why participants did not return the post-test questionnaires are unknown. We also changed the protocol for sending the T1 questionnaire to participants during the study. We noticed that it was more effective if the coach handed out the T1 questionnaire at the final group session, so that participants could return it during the individual outtake session. In addition, participants received a gift voucher if they had filled in all the questionnaires. The drop-out rate during the CooL programme was relatively low for both groups ( $18 \%$ for adults and $7 \%$ for children), and loss to follow-up for BMI was lower than for the questionnaires, as a result of our additional efforts to gather objective data.

During the study period, several actions were undertaken to increase the number of referrals, because in the early stage of the pilot project, referrals were slow to come in. First, the number of regions was extended. Second, the numbers of referrers in each region, such as the numbers of general practices, were increased. Third, various recruitment strategies were added. In the course of the pilot, proactive recruitment was also allowed, such as making use of social media, local newspapers, websites and electronic displays in the waiting rooms of general practices. Fourth, the inclusion period was extended by a year (until 1 April 2017).

\section{Unreported outcomes}

Not all the outcomes regarding various constructs mentioned in the study protocol have been described in the chapters of this thesis. During the study period, it became apparent that fewer completed questionnaires were returned than had been expected. Feedback from respondents taught us that the questionnaires were perceived as too long. As a result, we decided to reduce the length of the questionnaires, leading to a lack of follow-up data on constructs such as social support and self-efficacy in the adult questionnaire. Choices regarding the prioritisation of constructs in our research and analysis plan were based on our focus on motivational and behavioural changes. This also led to the decision to omit certain topics from the chapters in this thesis, such as the data on the children's home environment and body image. Future studies are planned to analyse these data.

Biomedical parameters of adults were collected by retrieving them from the general practitioners' information systems. These data were found to have many missing values, however, as the data appeared to be poorly recorded by the professionals. As a result, we were unable to reliably present these data in this thesis. 
Participants received a maximum of four different questionnaires (i.e. T0, T1, T2 and T3), as mentioned in the study protocol. For the analyses in Chapter 4 however, it was decided not to use the data of the T3 questionnaire (i.e. 24 months after baseline), because the T3 questionnaire did not contain all constructs that were relevant in that particular study (e.g. motivational regulation was not assessed, in order to reduce respondents' burden) and the sample size would have been smaller. As a result, the data of the T3 measurement have not been presented in this thesis, but they have been reported in the research report [78]. The results did not differ substantially from the results that are presented in this thesis.

\section{Internal and external validity}

The focus of the study was on internal as well as external validity. Several study components were designed to increase the internal validity, namely using a longitudinal study design, multiple qualitative and quantitative measurements, validated questionnaires and combining objectively measured BMI with self-reported BMI. The intention-to-treat principle was applied in analysing the quantitative data. This approach also takes into account the results of the participants who dropped out, as this is part of the intervention effect. A limitation of the study regarding internal validity was that not all the data were analysed with qualitative software programmes, due to the amount of data and the various types of qualitative data. Instead, paper-and-pen methodologies were used, while applying a coding system based on our research framework. Since our focus was on investigating the overall perspective, this approach suited the purpose of the study. Nevertheless, we had two researchers start the coding of the data, in order for them to agree on thematic codes and make the data analysis more reliable. Discrepancies between the two coders were discussed until agreement was reached. Additionally, with respect to the internal validity, the dynamics of the action-oriented nature of the study contributed to ad-hoc decisions regarding research focus and research methods, such as an increased focus on objectively measured BMI as the study progressed.

The factors of the study design that related to its external validity were the implementation in a real-world setting, the one-group pre-post design, and the adaptation of the programme's content. The study sample was representative of the Dutch population in that participation was free of charge. If CLIs will indeed be covered by health insurance policies from 2019 onwards, the CooL programme will also be offered free of charge at a national level, so this corresponds to the situation that was created for the participants in the pilot. It was also representative regarding the educational level of the participants, as the percentages of the various levels found in our study sample were similar to those in the Dutch population. Note that a relatively large part of the study sample had a low or intermediate educational level. By contrast, the study sample was not representative in terms of ethnicity and geography. Almost $95 \%$ of the adult study sample were of Dutch origin, whereas in the Netherlands as a whole, $76.9 \%$ of the population have a Dutch background [235]. Children were more representative of the national population, since $77.8 \%$ had a Dutch background. Furthermore, the CooL regions were only situated in two southern provinces of the Netherlands. 


\section{Health broker study}

A strength of the health broker study was the use of a theoretical framework to structure the interviews. Additionally, two researchers both coded three interviews and discussed the discrepancies between them with a third researcher until agreement had been reached. Another strength was that after the first analyses, the results were summarised for each participant and sent to them for a member check.

A limitation of the study was the selection of interviewees. We did not interview all relevant jobs involved in brokering, such as municipal authorities. In addition, on average only two professionals were interviewed for each type of job, which does not ensure data saturation. Since exploration was the specific goal of this study, it was sufficient to interview a relatively wide range of professionals to get a broad perspective, rather than to aim for data saturation and more in-depth information from one perspective (e.g. by focusing on health brokers only). Another limitation was that the data for the broker study were gathered approximately one year before the CooL study data were gathered. This may hamper efforts to compare or combine their results.

\section{IMPLICATIONS FOR PRACTICE}

\section{National development of CLIs and CooL}

In the course of the study period, it became clearer that CLIs were to be included in health insurance policies in the Netherlands from 2019 onwards. The new guidelines for CLIs describe the competences that professionals should have to be allowed to execute a CLI, but the type of professional is not defined. Health insurers can set up their own quality requirements for these professionals, based on the minimum required competences. The specific professionals or combination of professionals should at least have all the competences required to execute an effective $\mathrm{CLI}$ (i.e. comparable to a lifestyle coach having at least a degree from a university of applied sciences) [229]. The lack of specification in the new guidelines could result in many people wanting to function as lifestyle coaches without being sufficiently qualified. Hence, it is important to officially define the quality of lifestyle coaches and other professionals who are allowed to execute CLIs.

As a result of the action-oriented study described in this thesis, and to meet the new guidelines for CLIs, the protocol for CooL was further developed and adjusted by the lifestyle coaches [236]. The underlying determinants, theories and applications for this process have been described in this new protocol. In addition, some basic characteristics of the intervention were also changed. In the resulting new CooL programme, the programme period has been extended to two years, by combining the basic and relapse prevention programmes. Only if they have good reasons not to attend this relapse programme can participants stop after the basic programme. The programmes have now been described in more detail and an example script for each group session of the basic programme has been developed. Next to this, the attainment targets of the programme have been described to ensure the quality of the intervention. These targets include 
all topics that should have been addressed in the programme. The participants are assumed to be able to apply the topics described in the attainment targets to their own lifestyle. Finally, lifestyle coaches should register before being allowed to execute CooL, after a check to see whether they have the required competences as a coach. Any new Cool coach should attend a course. They also need to obtain accreditation points, have peer feedback meetings and monitor their participants' results on a yearly basis. The topics of the group sessions and the open character of the intervention have remained the same, as well as the number of sessions. However, positive health was also included in the programme [23]. CooL received an official accreditation ('first indications of effectiveness') from the Dutch Centre for Healthy Living, and is included in their Dutch database of best practice interventions [236]. This accreditation is required for the reimbursement of the programme by the health insurers.

\section{Further development of lifestyle coaching}

Lifestyle coaches could develop to take up the role of central care provider, by acting as a single point of contact regarding participants' lifestyle goals and by taking the lead among all care providers the participant has to deal with. Apart from coaching individuals towards a healthier lifestyle, they could then help people understand the information they receive and coordinate the process of care, including the involvement of the various specific professionals required to treat them [160]. Other studies are currently investigating the added value of such a central care provider and whether this role can be fulfilled by other professionals, such as YHC nurses $[237,238]$. It has been hypothesised that if more time is available to fulfil this role, patients would require less health care. YHC nurses could be good central care provider for children, whereas lifestyle coaches could adopt this role for adults.

A useful addition to the CooL programme could be the use of eHealth (i.e. electronic health) or mHealth (i.e. mobile health). Technological applications are rapidly developing all over the world. They can facilitate care on demand by means of easy exchange of information between the participants and their lifestyle coaches [239] as well as digital consultations, which may support the sustainability of the behaviour changes achieved. Examples include food diaries, physical activity monitors (such as the Fitbit), sleep trackers and chat functions. The benefits of adding these applications to the current programme are their ease of use, continuous availability and increased opportunities for gathering objective data. However, it should be taken into account that not all participants are able to use such applications and that the specific needs for eHealth or mHealth applications should be carefully studied from the end user perspective. Moreover, the professionals would need additional skills to use eHealth or mHealth applications properly.

\section{Context of prevention}

CooL applies to the prevention type of treatment and maintenance, targeting persons at increased weight-related risk. However, universal or indicated prevention, aiming to prevent people from becoming overweight or obese, would in the longer term be more 
efficient at national level. The coaches indicated that they would like to focus more on prevention, as this would benefit a larger part of the population. Although universal or indicated prevention may not be a suitable area for lifestyle coaches, an option would be to make the lifestyle coaches partners in indicated prevention by broadening the target group to include people who are overweight without having risk factors.

This preventive approach should be considered especially for children, in view of the complex situations of most families that include children with obesity. The professionals taking part in our study reported that there is a need for this preventive type of health care especially for children who have obesity or are at risk of it. Additionally, if children are overweight at a young age, there is a higher probability that their overweight will persist throughout their lives [240]. Therefore, starting obesity prevention at an early age by coaching the family of overweight children is to be recommended.

Not only lifestyle coaches, but also other health care professionals should focus more on prevention [217]. For instance, general practitioners can use a more preventive approach instead of a therapeutic approach by taking more time to give lifestyle advice or check for other possibilities [29]. Increased attention for lifestyle coaching already begins during medical training. Furthermore, the topic of lifestyle could be integrated in the training of other health care disciplines to reduce the disease burden and the risks of co-morbidity.

In addition to the greater focus on prevention, more complete integration between the different prevention levels should be achieved. Different sectors and professionals currently tend to focus more on the upper part of the prevention pyramid, whereas others focus more on the lower part of the pyramid (see Figure 1 in Chapter 1). A major cause is the funding system. The upper part of the pyramid is funded by the health insurance system in the Netherlands, while the lower part is funded by the municipal authorities or other public sector bodies. This creates a gap between the levels. Solutions to bridge this gap include changing funding structures, improving regulations, increasing intersectoral collaboration and integrated care by the various professionals, and encouraging professionals to look beyond their own professional field [212].

\section{Other public health problems}

The main topics in this thesis were overweight and obesity. However, this is only one of the topics that lifestyle coaching and health brokering could help to tackle; they could also be deployed for other public health problems. Lifestyle coaching, for instance, can also address many other chronic diseases such as cardiovascular disease, type 2 diabetes, lung diseases (such as chronic obstructive pulmonary disease), cancer, underweight and addiction (e.g. alcoholism and smoking), as they involve important lifestyle-related aspects. Health brokers also play a role in tackling other health problems. Their job starts with identifying the most important health problems within a region, which can range from socioeconomic health disparities (e.g. health literacy and low literacy), lifestyle or behavioural themes, mental problems and the physical environment (e.g. indoor environment), to loneliness and mobility problems among the elderly. Health bro- 
kers prioritise the different problems together with stakeholders, such as policy makers and citizens.

Similar to what happens with other problems, more attention could be given to subgroups of the target population, such as non-Dutch speaking persons and people with limited health literacy. Their needs should first be identified, so the coaching approach can be tailored to them and the programme can be adjusted.

\section{IMPLICATIONS FOR FURTHER RESEARCH}

One of the most important questions to be answered before adapting a programme to the different contexts is to know what the core components are (i.e. the essential and effective principles that make the programme effective) [155]. In our CooL study, several elements were systematically included in all the groups, for example a number of fixed themes. However, we did not investigate whether these components actually functioned as core components. Further research could address how the CooL intervention can be defined in terms of a fixed core and an adaptable periphery in relation to the context [241]. Additionally, it would be interesting to do further research to determine why participants started one of the supplementary programmes.

The position of lifestyle coaches seemed to be crucial for the implementation and success of the intervention. The network analyses we intend to perform in the context of the CooL study will help to investigate the development over time of the lifestyle coach's network in each region and their positioning. We hope to learn more about the successful characteristics of the networks and of the lifestyle coaches.

If CLIs are indeed included in health insurance policies, it is useful to continuously monitor the intervention outcomes, in order to evaluate the effects on a larger scale in a real-wolrd setting. Constructs that could be measured besides BMI are waist circumference, quality of life, lifestyle behaviours, co-morbidity and risk factors. What could also be studied are the effects among different subgroups of the target population, such as groups with a limited health literacy, groups with different weight-related health risks and different socio-economic groups. It is assumed that the inclusion of CLIs in health insurance policies will increase the awareness among referrers of the opportunities for referring their patients, which could result in a more heterogeneous group of participants. In addition to behavioural outcomes, the costs of national implementation should be investigated, in terms of health care and societal costs of the CLIs, compared with other health care methods. The estimated costs have already been calculated by means of a budget impact analysis [242]. The results show that CLIs will cost 5 million euro in 2019 and up to 15 million euro in the fifth year after the implementation of the new guidelines. Finally, it is important to evaluate the implementation process. Such an evaluation needs to relate successful implementation to the quality of the lifestyle coaches, the influence of the implementation context and the numbers of referrals. 
Apart from the CLIs themselves, the development of the bridging professionals within the overweight and obesity domains could be a subject for further studies. For instance, further research can focus on the added value of bridging professionals joining forces, their success rate within their networks and for closing the gap between prevention and health care domains.

\section{GENERAL CONCLUSION}

The findings of the studies described in this thesis allow the conclusion that professionals who function as linchpins within the field of prevention, policy and care (e.g. health brokers and lifestyle coaches) provide substantial added value in bridging the gap between the prevention and health care domains and in tackling complex problems such as obesity. We propose that collaborations between lifestyle coaches and health brokers are likely to result in more successful attempts to bridge this gap as regards obesity. A prerequisite is that their work should be complementary and should not overlap, for instance regarding collaboration with the same stakeholders.

Health brokers have shown their specific added value in bringing stakeholders together in terms of policy, practice and care, to address complex public health problems such as obesity. The CooL study provided the opportunity to evaluate the impact of the lifestyle coaches' role in the health care network and the public sectors. Concerning the adult target group, our research has shown the added value of lifestyle coaches in the health care chain for obesity. The coaches were found to be able to coach adult participants towards sustained behavioural change. As regards the children, no clear conclusions can be drawn regarding the added value. The findings of the CooL study suggest that the lifestyle coaches did not have enough entrepreneurial skills and motivation to intensively invest in creating and sustaining their professional network. They should become more visible as an important stakeholder in the obesity care for adults and children.

In the coming years, the Dutch prevention and care situation is expected to develop in such a way that lifestyle coaches will be able to assume a central position, together with other key parties, in order to optimise the integrated approach to combating obesity. 


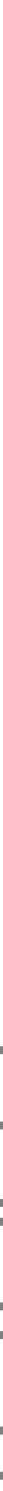




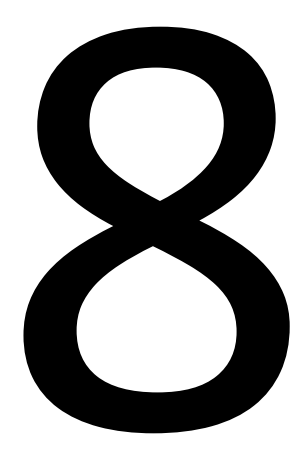

Appendix

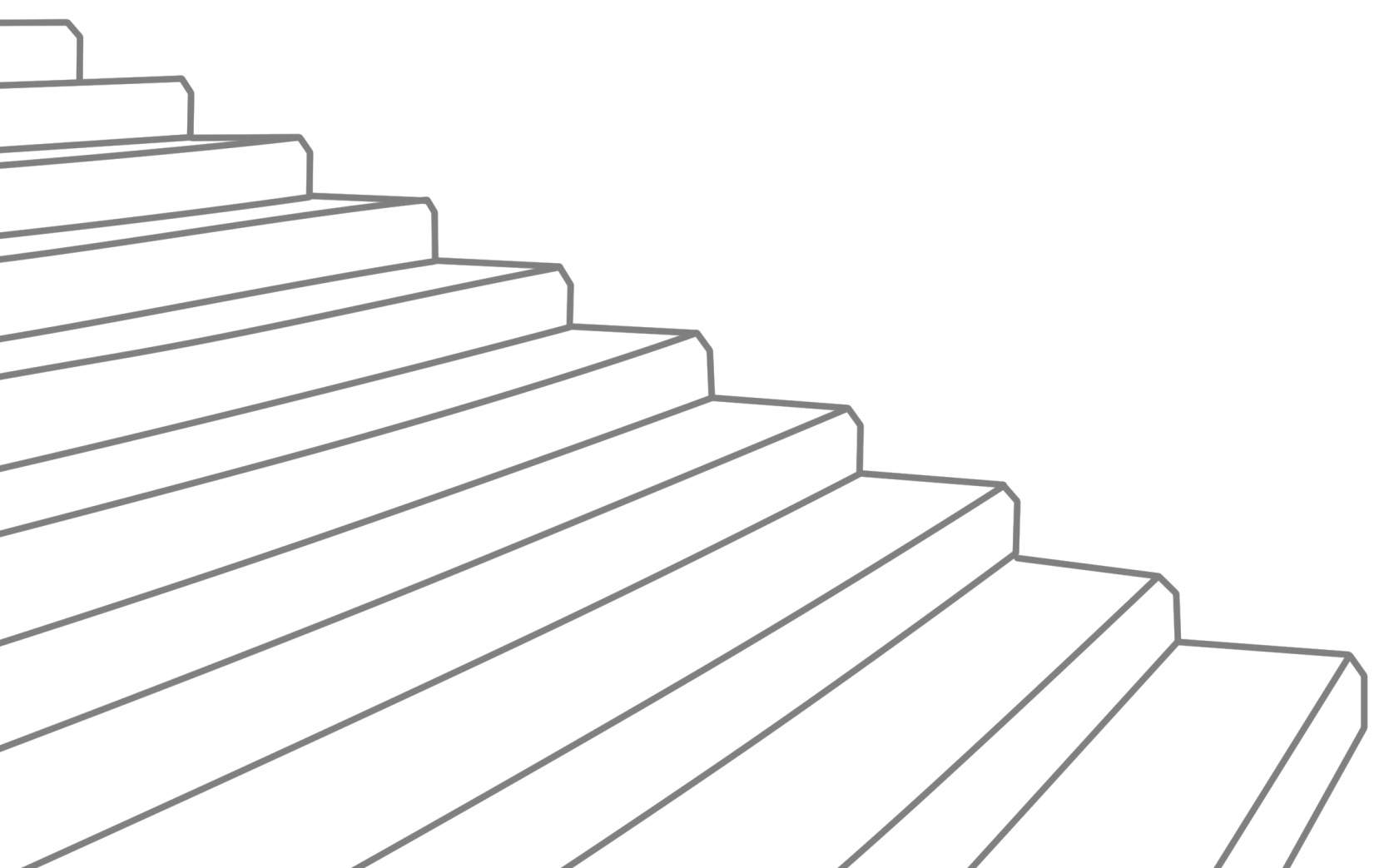





\section{VALORISATION}

An important aspect of scientific work, besides its value for the scientific community, is the translation of research findings into something with social impact. This chapter describes the relevance of the work presented in this thesis for society as a whole, including the target population, that is, people with obesity or at risk of it, lifestyle coaches, health care professionals, health brokers, health professionals, other bridging professionals and educational institutes. It also discusses how the findings of the studies described in this thesis can contribute to the work of other researchers.

\section{Relevance for society}

The studies presented in this thesis had a highly practical approach and focus, especially the Coaching on Lifestyle (CooL) study. This study evaluated the CooL intervention, in which people who are obese or at high risk of obesity are guided by a lifestyle coach. The topics in this combined lifestyle intervention (CLI) relate to obesity and lifestyle, including physical activity, dietary behaviours, sleep and stress management. This thesis has revealed the complexity of the implementation process of the CooL intervention, the facilitating and impeding factors in this process and the lifestyle changes achieved by the CooL participants over time.

CooL was implemented in a real-world setting to increase the chances of achieving sustainability of a nationally disseminated intervention programme. At the start of the implementation process in the pilot study, it was a programme that still needed further development. As a result of this, the research team and the stakeholders, including the lifestyle coaches who were the co-developers, encountered various real-world implementation barriers and were challenged to find ways to overcome these impeding factors. Furthermore, the action-oriented approach mean that the research team was in close contact with those who actually carried out the programme and other stakeholders. Thanks to this approach, this study was closely connected to the real-world setting in which the subsequent nation-wide implementation was expected to take place.

\section{Health care insurance}

The CooL intervention was started at the request of practitioners. The health insurance company $\mathrm{CZ}$ initiated the development and the evaluation of the CooL programme to improve participants' lifestyle. The goal of this pilot for $\mathrm{CZ}$ was to develop an optimal system for the reimbursement of CLIs by health insurance companies, with the ultimate aim of reducing the health care costs in the longer term. While our pilot study was underway, the Dutch government decided that CLIs will indeed be covered by the health insurance from 2019 onwards. This decision was partly based on the results of the studies described in this thesis. Since various parties were waiting to see the results of a successfully implemented $\mathrm{CLI}$, the CooL results came at exactly the right time.

The new guidelines for the reimbursement state that CLIs can be carried out by various professionals [229]. The specific professionals or combination of professionals involved 
should at least have all the competences required to execute an effective CLI, comparable with the competences of a lifestyle coach with at least a degree from a university of applied sciences. Individual health insurance companies are allowed to add additional quality requirements for these professionals, based on the minimum required competences.

Our studies found that the prospect that the new guidelines will apply from 2019 onwards had a positive influence on the motivation of the lifestyle coaches, referrers and other stakeholders in both the pilot regions and the regions that started the implementation after the pilot period.

\section{The population of people with obesity or at increased risk of it}

The implementation of the new guidelines and the reimbursement of CLIs by the health insurers from 2019 onwards is relevant for the Dutch population of people with obesity or at increased risk of it. It is expected that 3.5 million Dutch people will qualify for CLIs [242]. This number was calculated for the purpose of the nationwide implementation of $\mathrm{CLIs}$, in which the CooL research team assisted the authors, who performed the analysis [242].

The reimbursement of CLIs is very relevant for the target population. These people have usually tried to lose weight before, but are unable to maintain this weight loss due to motivational, habitual and contextual factors. This population will benefit from the support of a coach and of their peers in group sessions, as well as from the easy accessibility of reimbursed programmes. The fact that CLIs will be offered free of charge for participants will be even more welcome for people with a low socio-economic status. This target population has been difficult to reach over the last 35 years [243]. The CooL study has succeeded in including in its study sample a relatively large percentage with a low educational level. Nevertheless, reaching the populations that are most in need of it will remain a challenge for the nationally disseminated CLIs, and their success in this respect needs to be closely monitored.

\section{Further development of CooL into a programme protocol}

The fact that the lifestyle coaches were responsible for the further development of the content of the programme motivated them to design a detailed CooL programme protocol that meets the new national guidelines for CLIs. All qualified lifestyle coaches in the Netherlands can now buy the CooL programme materials from the company 'Expertisecentrum Leefstijlinterventies' (Expertise Centre for Lifestyle Interventions) and start it up in their own region. The basic programme consists of a format with an open character, meaning that lifestyle coaches can use the practical exercises and adapt them to what they feel comfortable with, and so as to fit the participants' needs. However, the attainment targets of the programme have been defined, to ensure the quality of the intervention. These targets include all topics that should be addressed in the programme. CooL received an official accreditation ('first indications of effectiveness') from the Dutch Centre for Healthy Living, and is included in their Dutch database of best 
practice interventions [236]. This accreditation is required for the reimbursement of the programme by the health insurers.

\section{Relevance for lifestyle coaches}

The CooL study and the national development of CLIs are also relevant for the profession of lifestyle coach. At the time the pilot started, in 2014, the lifestyle coach was a novel profession in the Dutch health care system. Over the course of the pilot period, lifestyle coaches became a more familiar phenomenon in the field of obesity prevention. The upcoming reimbursement of CLIs will probably lead to more people wanting to start with the CooL programme, increasing the need for coaches across the Netherlands.

The studies described in this thesis have provided lifestyle coaches in general with insights into what is relevant for executing their jobs. The action-oriented approach of the study enabled them to immediately integrate the knowledge they acquired in their routine practice. Coaching competences and empathy were found to be important competences for the coaches to have, and were well-developed among the coaches in this pilot. At the same time, it was found that the lifestyle coaches should further improve their entrepreneurial and networking skills, to create better stakeholder support, to increase the awareness of the concept of lifestyle coaches among professionals in the network and to create a sustainable professional network. Stakeholder support is necessary to successfully implement the intervention and to acquire a better position in the health care chain for tackling obesity.

In this light, coaches can take on the role of an 'ambassador' for the programme. If they cannot invest time in these networking activities themselves, or if they do not feel comfortable about doing so, other professionals can assist them. Additionally, lifestyle coaches could further develop the role of central care provider, by acting as a central point of contact regarding participants' lifestyle goals and by taking the lead among all care providers the participant has to deal with.

\section{Comparable programmes}

CooL formed a starting point for two other interventions, namely the healthyLIFE and Healthy Heart interventions. These two interventions differ slightly from CooL in terms of lifestyle coaching. After the CooL pilot study, one region (in the southern part of the Netherlands) started to implement CooL for adults, and further developed it into the healthyLIFE programme [244]. In healthyLIFE, they have integrated physical activity sessions with an exercise coach in the programme and provide several options for group size, i.e. a one-on-one programme, a buddy programme and a small group programme. Some activities are implemented during the lifestyle coaching sessions, e.g. supermarket safari, cooking workshop and walking activity.

The Healthy Heart intervention is executed by lifestyle coaches in one Dutch city (in the Randstad conurbation in the west of the Netherlands) [245]. The programme focuses not only on overweight, but also includes other lifestyle problems (e.g. smoking). Its 
study includes a control period, after which adults with an increased risk for cardiovascular diseases can start with the lifestyle programme. These two interventions were, like CooL, funded by the health insurance company CZ. More studies and a variety of programmes should create greater awareness of lifestyle coaching, and knowledge will be gained regarding effective coaching strategies.

Not only $\mathrm{CZ}$, but also other health insurance companies have initiated lifestyle programmes. An example is that the VGZ company funded the Keer Diabetes2 Om intervention [246]. In this programme various professionals (i.e. coach, dietician, nurse and programme coordinator) counsel patients with type 2 diabetes for half a year, starting with an intensive two-day programme. The development of these and similar initiatives will improve the scientific basis of the CLI.

\section{Health care context}

Not all health care professionals treat people with obesity the same as persons without obesity, due to prejudices, such as that obese patients are lazy and not motivated to try and improve their health [150]. They perceive it as a waste of time to treat these obese patients. Furthermore, at the time when our pilot started, obesity care was not a common theme to discuss during consultations in primary care [29]. Although the idea that obesity should be addressed in an integrated approach was gaining some ground, this happened at a very slow pace [14]. Integrated approaches to the prevention of chronic diseases have become more common since. Currently, health care professionals and policy makers are more aware of the importance of the impact of lifestyle behaviour on health outcomes [165].

Studies in this thesis could encourage health care professionals to acknowledge the relevance of their role in relation to obesity and prevention. First of all, these professionals have the possibility from 2019 onwards to refer obese patients to a reimbursed intervention, namely CooL or one of the other certified CLIs (currently BeweegKuur and SLIMMER). Second, health care professionals could take up their role in obesity prevention by using a more preventive approach instead of a curative approach, and by taking more time to motivate their patients and to give them lifestyle advice.

Furthermore, this research project can stimulate greater intersectoral collaboration between health care professionals and other stakeholders regarding obesity. Having more professionals with a bridging function in the health care chain would be beneficial for the intersectoral collaboration to prevent and treat obesity. Health brokers have shown their specific added value in bringing stakeholders together and facilitating intersectoral collaboration. They connect different stakeholders with each other, such as politicians, policy makers, health promotion practitioners, social and welfare workers, and private parties. A stronger collaboration between health brokers and lifestyle coaches can substantially enhance the integrated approach to reducing the burden of disease caused by unhealthy lifestyle. 
Relevance for health brokers and other bridging professionals

The 'Health Broker Wheel' model, which was developed in our health broker study, can be used by health brokers, health promotion practitioners or other 'bridges' as a tool to better implement their brokering role and to facilitate intersectoral collaboration. The tool can also help to monitor and evaluate this role. Health brokers can stimulate integrated care and collaboration within the wider local community. We hope that this study will encourage policy makers and practitioners to work together in a more integrated way.

\section{Relevance for educational institutes}

This research project had close connections with various training programmes. Coordinators of these training programmes can adjust their courses based on the insights provided in this thesis. Programmes for lifestyle coaches should focus more on their entrepreneurial and networking skills, as well as their role within the health care chain of obesity prevention. Furthermore, the courses for lifestyle coaches of children and adolescents should emphasise the complexity of childhood obesity problems and potential additional problems faced by their families. One of supervisors in this research project (SG) teaches in the training programme for paediatric lifestyle coaches at the Dutch Academy for Lifestyle and Health (AVLEG). During her involvement in the training programme, she also provided some insights from the research perspective. It is useful to provide training courses for lifestyle coaching at a high educational level, because of the complexity of the coaching skills and knowledge required.

Apart from the training programmes for lifestyle coaches, coaching skills should also be taught throughout all training courses for health care professionals such as dieticians, physiotherapists and general practitioners. Since many different types of professionals are potentially involved in CLIs, they should all have a high basic level of coaching skills. Additionally, not only lifestyle coaches, but also other health care professionals should focus more on prevention and on providing lifestyle advice. The topic of lifestyle should also be better integrated in the medical training of general practitioners and in the training of other health care disciplines.

Our health broker study resulted in collaboration between AVLEG and the main researcher to develop a new training programme for health brokers. It has already been investigated whether there is a need for such a programme and what it should contain, and further development work is being planned.

In addition, this thesis has stimulated discussions at Maastricht University about the competence profiles of students with regard to obesity-related topics. Master students should not only develop their scientific skills, but should also acquire practical skills, so as to prepare them for their professional life. The context of this type of public health problems is often complex, and there are no 'magic bullet' solutions available for these 'wicked' problems. These problems therefore need to be approached in a flexible way, applying adaptive management strategies (i.e. adapted to the local context). This the- 
sis may help to further stimulate improvements in the education of students, teaching them how to deal with wicked problems and complex contexts.

\section{Relevance for researchers}

The action-oriented study design of the CooL study is useful for researchers who want to study an intervention in a complex real-world setting. A strength of this approach was that it resulted in close and effective collaboration between the lifestyle coaches and the researchers, which enabled the implementation process to be closely followed and improved when needed. However, limitations of this design included taking ad-hoc decisions and the challenge for the researchers to maintain an objective view. Furthermore, the differences between the ways the coaches executed the programme could not be investigated systematically, because of the continuing changes to the programme over time. As a follow-up to the CooL study, two PhD students have initiated studies to further improve the contents and monitoring of CLIs.

The Dutch national media, such as newspapers (AD, De Limburger and Trouw), websites and social media, were interested in the CooL pilot from the beginning, and closely followed the developments of the pilot study and the intervention. We learned that it is useful to have a contact person at a newspaper and to use these contacts, rather than sending out general press releases. We also found out that the title of a newspaper article will only be established at the moment of publicaton, since it is written by the subeditor. The resulting heading can have considerable impact on the way the article is perceived by the readers, so it is advisable to provide a suggestion for a title when reviewing the draft version of the article.

We expect our recommendations throughout this thesis to be helpful in improving the dissemination, recruitment and monitoring of CLIs. If CLIs are reimbursed by health insurance, it will be useful for researchers to continuously monitor the intervention outcomes, in order to evaluate the long-term effects on a larger scale in a real-world setting. In addition, the costs of national implementation should be investigated, in terms of health care and societal costs of the CLIs, compared with other health care methods. Finally, it is important to evaluate the implementation process, in view of the changes taking place in the health care and prevention chain, and the time that is needed to implement the intervention at the national level. Such an evaluation should also investigate the influence of the implementation context. 




\section{SUMMARY}

The prevalence of overweight and obesity has reached alarming proportions worldwide, including in the Netherlands. Due to the complexity of the obesity problem, a combination of different strategies addressing various health determinants is required to tackle it. The studies reported on in this thesis focused on potential solutions at different prevention levels, ranging from collective prevention to intensive treatment. The central research question was: Can lifestyle coaches and health brokers help to bridge the gap between the prevention and health care domains regarding overweight and obesity? Several professionals can play important roles in bridging this gap by adopting a central position in the network of professionals. Two types of professionals have been studied in relation to this brokering function: lifestyle coaches and health brokers.

\section{Chapter 2: study protocol of the CooL intervention}

Lifestyle coaches can play a brokering role in obesity prevention as they have close contacts with the target group and with professionals in the network of obesity care and prevention. The role of lifestyle coaches is a new one in the Dutch health care system. In the Coaching on Lifestyle ( $\mathrm{CooL}$ ) intervention, they counsel adults and children aged 4 years and older (and their parents) towards a healthier lifestyle. The inclusion criterion for the intervention is being obese or overweight with an increased risk of developing cardiovascular diseases or type 2 diabetes. The CooL intervention is a combined lifestyle intervention (CLI). Previous CLIs have proved to be effective in changing and maintaining healthier lifestyle behaviours and reducing overweight and obesity, in clinical as well as community settings.

The themes addressed in the CooL intervention are similar to those in regular CLIs: physical activity, dietary and behavioural components, with additional topics of sleep and stress management. Over a period of 8 to 10 months, lifestyle coaches use group and individual sessions to promote sustainable lifestyle changes among the participants. Furthermore, the coaches encourage the participants to take responsibility for their own behaviour. The participants are helped to set challenging but achievable goals for each of the above behaviours, which is expected to improve their long-term health. If participants who have completed the basic programme are found to be still in need of further counselling, they can enter one of two supplementary programmes (i.e. a relapse prevention programme and an additional programme). The intervention is reimbursed by health insurance companies and therefore free of charge for participants.

The aim of the CooL study was to monitor the implementation process of the Cool intervention and to examine how the lifestyle coaches contribute to a healthier lifestyle of the participants. The process was studied by means of semi-structured group and individual interviews, observations and document analysis (qualitative research). The lifestyle changes over time among participants were examined by means of questionnaires and objective parameters (quantitative research), in a one-group pre-post design. Furthermore, the study applied an action-oriented approach, meaning that the research team was closely involved in the implementation process. Since the main re- 
searcher participated in all organisational meetings, this enabled her to observe and simultaneously support the implementation process.

\section{Chapter 3: summary of the results from the CooL intervention}

This chapter presents a summary of the results of an evaluation of the CooL intervention; these results are described in more detail in the following two chapters. The CooL study has shown that the CooL intervention had potential in coaching adult participants towards sustained behavioural change. The findings also showed that lifestyle coaches can fulfil an important role in connecting the various links in the chain of professionals addressing the problem of overweight in the Netherlands, especially regarding the adult target group. Lifestyle coaches provide easily accessible guidance towards sustainable behavioural change, as well as connecting professionals in the care and public domains, and referring participants to other relevant services. As regards the target group of children and adolescents, the findings do not as yet allow definitive conclusions to be drawn regarding the potential added value offered by integrating lifestyle coaches in the chain of care, as the sample size was too small. Furthermore, behavioural change proved to be difficult to achieve within the study period, due to the often complex family situations.

The CooL study offers useful insights which can help to sustainably incorporate lifestyle coaching in the integrated approach to obesity. A number of recommendations have been identified for further optimisation of the intervention. The intervention should be further developed as regards interdisciplinary collaboration, a number of the coaches' competences (especially those concerning referrals towards other professionals, 'entrepreneurship', and counselling children and their parents), creating a more professional organisation (including quality assurance by means of peer feedback and supervision) and further elaboration of the relapse prevention programme. In addition, it would seem advisable to not only use weight loss as an outcome measure, but also to focus on lifestyle-related indicators, as the CooL approach involves small action steps for behavioural change. As regards the possible implementation on a national scale, the pilot project has shown that it is important to take enough time to inform referrers and lifestyle coaches, and bring them into contact with each other, so they can find out each other's expertise and learn from each other's positive and negative experiences.

\section{Chapter 4: longitudinal lifestyle changes of CooL participants}

In a longitudinal one-group pre-post study, the motivational, behavioural and weight changes among the participants of the CooL intervention were monitored over a 1.5 year period. Favourable and sustained changes among adults were found regarding perceived autonomy, motivation, perceived barriers, lifestyle behaviours, quality of life and weight. Adults lost an average of $2.3 \mathrm{~kg}$ of body weight, which was largely maintained approximately one year later.

The participating children and their parents, however, showed smaller and less significant changes. Significant improvements were found regarding the time spent watching 
television, dietary behaviours, the quality of life scales physical comfort and the overall score. In contrast, the children developed a significantly more negative attitude towards fruit consumption. In general, there were no significant changes regarding the children's physical activity, motivation, habit formation or BMI z-score. Their parents, however, perceived fewer problems with their child's behaviour, they increased their self-efficacy as regards addressing these issues, and they improved some of their parenting practices regarding healthy eating and physical activity.

It can be concluded that the lifestyle coaches in the CooL intervention have been successful in coaching adult participants towards sustained behavioural change during the intervention period, while mixed results and smaller changes were found for children and their parents.

\section{Chapter 5: process evaluation of the CooL implementation}

The aim of this study was to evaluate the implementation process of the CooL intervention and its facilitating and impeding factors. The CooL intervention was implemented in multiple regions, involving the efforts of many stakeholders. The intervention was evaluated by stakeholders (e.g. the project team members) and participants as an accessible and useful intervention, thanks to its design and content and the lifestyle coaches' approach. However, stakeholders indicated that the lifestyle coaches needed to become more visible in the health care network and public sectors in the Netherlands. Lifestyle coaching is a novel profession and the added value of the lifestyle coach is not always acknowledged by all health care providers. Lifestyle coaches play a crucial role in ensuring the impact of the CooL intervention by actively networking, using clear communication materials and creating stakeholder support and understanding.

A substantial number of barriers have been overcome (such as dealing with resistance from referrers), and promising opportunities have arisen for integrating lifestyle coaching in a broader approach, based on the coaches' central role, in order to bridge the gap between prevention and treatment of chronic diseases. However, the implementation and dissemination processes need to be more effectively executed, including creating support for lifestyle coaching and providing clear information. Networking activities should be intensified and the contents of the intervention continuously improved to fit the implementation settings. It will take time before the lifestyle coaches have become accepted as valuable professionals who bridge the gap between the public health sector and health care settings.

\section{Chapter 6: behaviours and determinants of the health broker's role}

Although health brokers have been active for some time in some regions in the Netherlands, the role of health broker, just like that of the lifestyle coach, is a relatively new phenomenon in the public health sector. Health brokers aim to create support for efforts to optimise health promotion in complex public health contexts by facilitating intersectoral collaboration and by exchanging knowledge with different stakeholders. The current study aimed to explore the role of health brokers regarding emerging complex 
public health problems, by examining the motivational, contextual and behaviour-related factors the health brokers have to deal with.

Fifteen professionals from various backgrounds and from various policy and practice organisations were recruited for semi-structured interviews. A theoretical framework called the 'Health Broker Wheel' was developed to visualise the health brokers' role.

Seven main types of behaviour that health brokers need to engage in were identified: recognising opportunities, agenda setting, implementing, network formation, intersectoral collaboration, adaptive managing and leadership. The most important behaviour expected of health brokers consists of facilitating intersectoral collaboration, for example between policy and practice, in order to improve public health. Determinants of health brokers' behaviours were identified and categorised as capability, opportunities, motivation, and local or national contextual factors.

The findings showed that health brokers can make useful contributions to addressing complex public health problems, such as obesity. These problems require intersectoral collaboration and adapting to contextual factors. Health brokers operate as 'anchoring points' in connecting community problems to policies and services. They thus represent a good example of bringing together research evidence, health policy and practice in addressing complex public health problems.

\section{Chapter 7: general discussion}

The findings of the studies presented in this thesis are summarized in the general discussion. In addition, methodological considerations are discussed and implications for practice and further research are suggested. The main conclusion of this thesis is that lifestyle coaches have shown their added value in fulfilling the brokering role in the health care chain of obesity, definitely as regards the adult target group. As regards the population of children, no clear conclusions can be made regarding their added value. Health brokers have shown their added value in presenting a good example of bringing stakeholders together to address complex public health problems such as obesity. It is proposed that collaboration between lifestyle coaches and health brokers is likely to result in more successful attempts to bridge the gap between prevention and health care concerning obesity. 




\section{SAMENVATTING}

Overgewicht en obesitas komen wereldwijd en in Nederland steeds vaker voor. Door de complexiteit van het obesitasprobleem is een combinatie van verschillende strategieën, gericht op meerdere gezondheidsdeterminanten, nodig om het probleem tegen te gaan. De studies in dit proefschrift waren gericht op oplossingen op verschillende preventieniveaus, variërend van universele tot zorg-gerelateerde preventie. De centrale onderzoeksvraag was: Kunnen leefstijlcoaches en gezondheidsmakelaars helpen om de kloof tussen de preventie en zorg met betrekking tot overgewicht en obesitas te overbruggen? Verschillende professionals kunnen een belangrijke rol spelen in het overbruggen van deze kloof door een centrale positie te nemen in het netwerk van professionals die zich bezighouden met obesitaspreventie. Twee soorten professionals zijn onderzocht in relatie tot deze brugfunctie: leefstijlcoaches en gezondheidsmakelaars.

\section{Hoofdstuk 2: onderzoeksprotocol van de CooL-interventie}

Leefstijlcoaches kunnen een brugfunctie vervullen in obesitaspreventie, omdat zij de mogelijkheid hebben om als zorgverleners nauw in contact te zijn met de doelgroep en professionals in het netwerk van obesitaszorg en -preventie. De leefstijlcoach is een nieuwe professional in het Nederlandse gezondheidzorgsysteem. In de Coaching op Leefstijl (CooL) interventie begeleiden zij volwassenen en kinderen vanaf 4 jaar en ouder (en hun ouders) naar een gezondere leefstijl. Het inclusiecriterium voor de interventie is het hebben van obesitas óf overgewicht met daarbij een verhoogd risico op het ontwikkelen van hart- en vaatziekten of diabetes type 2 . CooL is een gecombineerde leefstijlinterventie (GLI). Voorgaande GLI's hebben bewezen effectief te zijn in het veranderen en behouden van leefstijlgedragingen en in het verminderen van overgewicht en obesitas, in gecontroleerd onderzoek en in de praktijk.

De thema's behandeld in de CooL interventie zijn vergelijkbaar met de reguliere GLI-thema's: lichamelijke beweging, voeding en gedrag, aangevuld met de thema's slaap en stressmanagement. In groeps- en individuele sessies, in het 8 tot 10 maanden durende basisprogramma, coachen leefstijlcoaches de deelnemers om hun leefstijl duurzaam te veranderen. De leefstijlcoaches stimuleren deelnemers om verantwoordelijkheid te nemen voor hun eigen gedrag. Daarnaast worden de deelnemers ondersteund om uitdagende maar haalbare doelen te stellen voor elk van de bovengenoemde gedragingen, die hun gezondheid op lange termijn kunnen verbeteren. Als deelnemers het basisprogramma hebben afgerond en nog steeds behoefte hebben aan verdere begeleiding, kunnen zij in één van de twee aanvullende programma's instromen (namelijk een terugval- of aanvullende programma). De interventie wordt vergoed door zorgverzekeraars en deelname is daardoor gratis voor deelnemers.

Het doel van het CooL-onderzoek was het monitoren van het implementatieproces van de CooL-interventie en onderzoeken of de leefstijlcoaches bijdragen aan een gezondere leefstijl van de deelnemers. Het proces werd bestudeerd aan de hand van semigestructureerde groeps- en individuele interviews, observaties, en documentanalyse (kwalitatief onderzoek). De leefstijlveranderingen van deelnemers over de tijd waren 
onderzocht met behulp van vragenlijsten en objectieve parameters (kwantitatief onderzoek), in een pre-post design zonder controlegroep. Daarnaast had de studie een actie-begeleidende aanpak, hetgeen betekent dat het onderzoeksteam nauw betrokken was bij het implementatieproces. De hoofdonderzoeker nam deel aan alle organisatorische overleggen, wat haar in staat stelde om het implementatieproces te observeren en gelijktijdig te ondersteunen.

\section{Hoofdstuk 3: samenvatting van de resultaten van de CooL-interventie}

In dit hoofdstuk worden de resultaten van de evaluatie over de CooL-interventie samengevat en in de volgende twee hoofdstukken worden de resultaten uitgebreider beschreven. Uit het CooL-onderzoek blijkt dat de CooL-interventie potentie heeft om volwassen deelnemers te begeleiden naar duurzame gedragsverandering. Leefstijlcoaches kunnen een belangrijke rol vervullen in het verbinden van de verschillende professionals in de keten die het overgewichtprobleem in Nederland aanpakken, voornamelijk met betrekking tot de volwassen doelgroep. De begeleiding van leefstijlcoaches naar een duurzame gedragsverandering is toegankelijk gebleken. Daarnaast zijn de leefstijlcoaches in de positie waarin ze kunnen dienen als een verbinder tussen professionals in de zorg- en het publieke domein en ze gemakkelijk deelnemers naar andere relevante diensten kunnen doorverwijzen. Echter laten de onderzoeksbevindingen zien dat leefstijlcoaches de verbindende rol alleen in beperkte mate uitvoeren. Wat betreft de doelgroep kinderen en adolescenten is het aan de hand van het huidige onderzoek niet mogelijk om definitieve conclusies te trekken over de potentiële toegevoegde waarde van leefstijlcoaches in de ketenzorg, omdat de studiepopulatie te klein was. Daarnaast veronderstellen we dat gedragsverandering lastig te behalen is binnen de onderzoeksperiode, vanwege de vaak complexe familiesituaties.

De CooL-studie bood nuttige inzichten om leefstijlcoaching duurzaam te integreren in de integrale aanpak van obesitas. Een aantal aanbevelingen werd gedaan om de interventie verder te optimaliseren. De interventie moet worden doorontwikkeld met betrekking tot interdisciplinaire samenwerking, een aantal competenties van de leefstijlcoaches (vooral wat betreft het doorverwijzen naar andere professionals, 'ondernemerschap' en het begeleiden van kinderen en hun ouders), professionalisering van de organisatie omtrent leefstijlcoaching (inclusief kwaliteitsborging door middel van intervisie en supervisie) en het verder uitwerken van het terugvalprogramma. Daarnaast lijkt het raadzaam om niet alleen gewichtsverlies als uitkomstmaat te gebruiken, maar ook te focussen op leefstijl-gerelateerde indicatoren, omdat de CooL-aanpak gericht is op het zetten van kleine stappen van gedragsverandering. Wat betreft de mogelijke implementatie op landelijk niveau heeft deze pilot aangetoond dat het belangrijk is om voldoende tijd te nemen om verwijzers en leefstijlcoaches te informeren en om ze met elkaar in contact te brengen, zodat ze elkaars expertise beter kunnen begrijpen en kunnen leren van elkaars positieve en negatieve ervaringen. 


\section{Hoofdstuk 4: longitudinale leefstijlveranderingen van CooL-deelnemers}

In een longitudinale pre-poststudie met één groep werden de motivatie, gedrag, gewicht en kwaliteit van leven van de deelnemers van de CooL-interventie gevolgd gedurende 1,5 jaar. Positieve en duurzame veranderingen werden bij volwassenen gevonden met betrekking tot autonomie, motivatie, barrières, leefstijlgedrag, kwaliteit van leven en gewicht. Volwassenen verloren gemiddeld 2,3 kg lichaamsgewicht. Eén jaar na afronding van de interventie was het resultaat op gewichtsverlies nog steeds zichtbaar.

Deelnemende kinderen en hun ouders vertoonden echter minder en kleinere veranderingen. Significante verbeteringen werden gevonden met betrekking tot de tijd dat kinderen televisiekeken, de voedingsinname en de kwaliteit van leven schalen lichamelijk ongemak en de totaalscore. Daarentegen ontwikkelden de kinderen een significant negatievere attitude ten aanzien van fruitconsumptie. Over het algemeen waren er geen significante veranderingen gevonden bij de kinderen met betrekking tot het beweeggedrag, motivatie, gewoontevorming of BMI z-score. Hun ouders ervoeren echter minder problemen met het gedrag van hun kind, ze verhoogden hun eigen effectiviteit wat betreft het aanpakken van deze problemen en ze verbeterden enkele ouderschapspraktijken ten aanzien van voeding en bewegen.

Het kan geconcludeerd worden dat de leefstijlcoaches in deze CooL-interventie succesvol zijn geweest in het coachen van volwassen deelnemers naar een duurzame gedragsverandering tijdens de interventieperiode, terwijl gemengde resultaten en kleinere veranderingen voor kinderen en hun ouders werden gevonden.

\section{Hoofdstuk 5: procesevaluatie van de CooL-implementatie}

Het doel van deze studie was om het implementatieproces van de CooL-interventie en de daarbij behorende faciliterende en belemmerende factoren te evalueren. De CooL-interventie werd uitgevoerd in meerdere regio's, dankzij de inspanningen van vele belanghebbenden. De interventie werd door belanghebbenden (zoals leden van de projectgroepen) en deelnemers beoordeeld als een toegankelijke en nuttige interventie, vanwege de opzet en de inhoud van de interventie en de aanpak van de leefstijlcoaches. Belanghebbenden gaven echter aan dat de leefstijlcoaches meer bekendheid moesten krijgen in het zorgnetwerk en publieke sectoren in Nederland. Leefstijlcoaching is een nieuw beroep en de toegevoegde waarde van de leefstijlcoach wordt niet altijd erkend door alle zorgverleners. Leefstijlcoaches spelen een cruciale rol bij het waarborgen van de impact van de CooL interventie door actief te netwerken, gebruik te maken van helder communicatiemateriaal en het creëren van draagvlak en begrip van belanghebbenden.

Een aanzienlijk aantal barrières is overwonnen (zoals het omgaan met de weerstand van verwijzers) en er zijn veelbelovende mogelijkheden ontstaan voor het integreren van leefstijlcoaching in een bredere aanpak door de centrale rol van de coaches, om de kloof tussen preventie en zorg van chronische ziekten te overbruggen. De implementatie- en disseminatieprocessen moeten echter effectiever worden voorbereid en 
uitgevoerd, inclusief het creëren van draagvlak voor leefstijlcoaching en het verstrekken van duidelijke informatie. Netwerkactiviteiten moeten frequenter plaatsvinden en de inhoud van de interventie moet voortdurend worden verbeterd, om aan te sluiten bij de implementatie settings. Het zal enige tijd duren voordat de leefstijlcoaches geaccepteerd zijn als waardevolle professionals die trachten de kloof tussen de publieke gezondheidssector en de zorgsettingen te overbruggen.

\section{Hoofdstuk 6: gedrag en determinanten van de rol van de gezondheidsma- kelaar}

Hoewel in enkele regio's in Nederland gezondheidsmakelaars al enige tijd actief zijn, is de rol van gezondheidsmakelaar, net als de leefstijlcoach, relatief nieuw fenomeen in de gezondheidssector. Gezondheidsmakelaars streven ernaar om draagvlak te creëren voor het optimaliseren van gezondheidsbevordering in complexe gezondheidscontexten door intersectorale samenwerkingsverbanden mogelijk te maken en door kennis uit te wisselen met verschillende belanghebbenden. Het doel van de gezondheidsmakelaar studie was om de rol van gezondheidsmakelaars te onderzoeken bij opkomende complexe gezondheidsproblemen door de motivationele, contextuele en gedrags-gerelateerde factoren te achterhalen waarmee de ze te maken hebben.

Vijftien professionals met verschillende achtergronden en van verschillende beleids- en praktijkorganisaties werden geworven voor semigestructureerd interviews. Een theoretische kader genaamd 'Health Broker Wheel' werd ontwikkeld om de rol van de gezondheidsmakelaar te visualiseren.

Er werden zeven hoofdgedragingen geïdentificeerd waar gezondheidsmakelaars zich mee bezig houden: herkennen van mogelijkheden, agenderen, implementeren, netwerk formeren, intersectoraal samenwerken, adaptief managen en het tonen van leiderschap. Het belangrijkste gedrag dat wordt verwacht van gezondheidsmakelaars is het faciliteren van intersectorale samenwerking, bijvoorbeeld tussen beleid en praktijk om de volksgezondheid te verbeteren. Determinanten van het gedrag van gezondheidsmakelaars werden geïdentificeerd en gecategoriseerd als vaardigheden, mogelijkheden, motivatie en lokale of nationale contextuele factoren.

De bevindingen toonden aan dat gezondheidsmakelaars een nuttige bijdrage kunnen leveren bij het aanpakken van complexe gezondheidsproblemen, zoals obesitas. Deze problemen vereisen intersectorale samenwerking en het aanpassen aan de contextuele factoren. Gezondheidsmakelaars fungeren voornamelijk als 'spin in het web', waarbij ze de problemen in de samenleving verbinden met beleidsmaatregelen en diensten. Daarmee zijn ze een goed voorbeeld van hoe wetenschappelijke kennis, gezondheidsbeleid en praktijk samengebracht kunnen worden bij het aanpakken van complexe gezondheidsproblemen. 


\section{Hoofdstuk 7: algemene discussie}

De bevindingen van alle studies in dit proefschrift zijn samengevat in de algemene discussie. Verder worden methodologische overwegingen besproken en worden implicaties voor de praktijk en voor vervolgonderzoek voorgesteld. De hoofdconclusie van dit proefschrift is dat leefstijlcoaches hun toegevoegde waarde hebben laten zien in de zorg rondom obesitas, zeker met betrekking tot de volwassen doelgroep. Wat betreft de kinderpopulatie kunnen er geen duidelijke conclusies worden getrokken over hun toegevoegde waarde. Gezondheidsmakelaars hebben hun toegevoegde waarde laten zien als een goed voorbeeld van het samenbrengen van belanghebbenden bij het aanpakken van complexe gezondheidsproblemen, zoals obesitas. Samenwerking tussen leefstijlcoaches en gezondheidsmakelaars zal mogelijk resulteren in meer succesvolle pogingen om de kloof tussen preventie en gezondheidszorg met betrekking tot obesitas te dichten. 



\section{REFERENCES}

1. World Health Organisation. Obesity and overweight. 2017. http://www.who.int/mediacentre/factsheets/fs311/en/. Accessed on 26 Jan 2016.

2. NCD Risk Factor Collaboration. Trends in adult body-mass index in 200 countries from 1975 to 2014 : a pooled analysis of 1698 population-based measurement studies with 19.2 million participants. The Lancet. 2016;387(10026):1377-1396.

3. Dobbs R, Sawers C, Thompson F, Manyika J, Woetzel J, Child P, et al. Overcoming obesity: An initial economic analysis. London: McKinsey Global Institute; 2014.

4. Centraal Bureau voor de Statistiek. Lengte en gewicht van personen, ondergewicht en overgewicht; vanaf 1981 [Height and weight of persons, underweight and overweight; since 1981]. Den Haag/Heerlen, 2018. https://opendata.cbs.nl/\#/CBS/nl/dataset/81565NED/table?ts=1530885747034. Accessed on 6 Jul 2018.

5. World Health Organization. Tenfold increase in childhood and adolescent obesity in four decades: new study by Imperial College London and WHO. 2017. http://www.who.int/mediacentre/news/releases/2017/increase-childhood-obesity/en/. Accessed on 20 Oct 2017.

6. The GBD 2015 Obesity Collaborators. Health Effects of Overweight and Obesity in 195 Countries over 25 Years. New England Journal of Medicine. 2017;377(1):13-27.

7. World Health Organization. Obesity: preventing and managing the global epidemic. Report of a WHO Consultation. Geneva; 2000.

8. Kremers SP, Visscher TL, Seidell JC, van Mechelen W, Brug J. Cognitive determinants of energy balance-related behaviours: measurement issues. Sports Med. 2005;35(11):923-933.

9. Chau JY, Grunseit AC, Chey T, Stamatakis E, Brown WJ, Matthews CE, et al. Daily Sitting Time and AllCause Mortality: A Meta-Analysis. PLoS One. 2013;8(11):e80000.

10. Teixeira PJ, Silva MN, Mata J, Palmeira AL, Markland D. Motivation, self-determination, and long-term weight control. International Journal of Behavioral Nutrition and Physical Activity. 2012;9(1):22.

11. Van Mil E, Struik A. Overgewicht en obesitas bij kinderen, verder kijken dan de kilo's [Overweight and obesitas in children, looking beyond the kilograms]. Amsterdam: Uitgeverij Boom; 2015.

12. Capers PL, Fobian AD, Kaiser KA, Borah R, B.Allison D. A Systemic Review and Meta-Analysis of Randomized Controlled Trials of the Impact of Sleep Duration on Adiposity and Components of Energy Balance. Obesity Reviews. 2015;16(9):771-782.

13. Perry KI, MacDonald SJ. The obese patient: a problem of larger consequence. The Bone \& Joint Journal. 2016;98-b(1 Suppl A):3-5.

14. Partnerschap Overgewicht Nederland. Zorgstandaard Obesitas [Care Guidelines for Obesity]. Amsterdam: Partnerschap Overgewicht Nederland; 2010.

15. Lang T, Rayner G. Overcoming policy cacophony on obesity: an ecological public health framework for policymakers. Obesity Reviews. 2007;8(s1):165-181.

16. Helmink JHM, Raaijmakers LGM, Rutten GM, Vries NK, Kremers SPJ. Gecombineerde leefstijl interventies in Nederland: ervaringen uit de BeweegKuur [Combined lifestyle interventions in the Netherlands: experiences gained with the BeweegKuur intervention]. Tijdschrift voor Gezondheidswetenschappen. $2013 ; 2013$.

17. Dietz WH, Baur LA, Hall K, Puhl RM, Taveras EM, Uauy R, et al. Management of obesity: improvement of health-care training and systems for prevention and care. The Lancet. 2015;385(9986):2521-2533.

18. Ministerie van Volksgezondheid Welzijn en Sport. Langer gezond leven 2004-2007; ook een kwestie van gezond gedrag [A longer healthy life 2004-2007; also a question of healthy behaviour]. Den Haag; 2003.

19. Ministerie van Volksgezondheid Welzijn en Sport. Kiezen voor gezond leven 2007-2010 [Opting for a healthy life 2007-2010]. Den Haag; 2006.

20. Rijksoverheid. Alles is gezondheid... Het Nationaal Programma Preventie 2014 - 2016 [Everything is health... The National Prevention Programme 2014 - 2015]. Den Haag; 2013.

21. Van der Veen, Joldersma, Vendrik, Voordewind, Van der Vlies. Motie 30800 XVI 74 [Motion by members of parliament], (2007).

22. Ministerie van Volksgezondheid Welzijn en Sport. Kamerbrief Landelijke nota gezondheidsbeleid 20162019 [Letter to parliament on national health policy document 2016-2019], 846306-142553-PG (2015).

23. Huber M, Knottnerus JA, Green L, Horst Hvd, Jadad AR, Kromhout D, et al. How should we define health? BMJ. 2011;343.

24. Gezondheidsraad. Richtlijnen goede voeding 2015 [Guidelines on healthy nutrition 2015]. Den Haag; 2015.

25. Gezondheidsraad. Beweegrichtlijnen 2017 [Physical activity guidelines 2017]. Den Haag; 2017. 
26. Timmermans O. Crossing over to a healthy region [Inaugural address]. Vlissingen: HZ University of Applied Sciences; 2015.

27. Bouma AJ, van Wilgen P, Baarveld F, Lemmink KAPM, Diercks RL, Dijkstra A. A Cross-sectional Analysis of Motivation and Decision Making in Referrals to Lifestyle Interventions by Primary Care General Practitioners: A Call for Guidance. American Journal of Lifestyle Medicine. 2017.

28. Seidell JC, Halberstadt J, Noordam H, Niemer S. An integrated health care standard for the management and prevention of obesity in The Netherlands. Family Practice. 2012;29(suppl 1):i153-i156.

29. Van Dillen SME, van Binsbergen JJ, Koelen MA, Hiddink GJ. Nutrition and physical activity guidance practices in general practice: A critical review. Patient Education and Counseling. 2013;90(2):155-169.

30. Van Dillen SM, Noordman J, Van Dulmen S, Hiddink GJ. Setting goal and implementation intentions in consultations between practice nurses and patients with overweight or obesity in general practice. Public Health Nutrition. 2015;18(16):3051-3059.

31. Nederlands Centrum Jeugdgezondheid. JGZ-richtlijn. Overgewicht: preventie, signalering, interventie en verwijzing [Youth Health Care guidelines. Overweight: prevention, signaling, intervention and referring]. Utrecht; 2012.

32. Halberstadt J, Seidell J. Addendum ernstige kinderobesitas bij de Zorgstandaard Obesitas. Ketenzorg voor kinderen met een extreem verhoogd gewichtsgerelateerd gezondheidsrisico en hun ouders [Addendum on severe childhood obesity in the health care guideline on obesity. Integrated care for children with a very severely increased weight-related health risk and their parents]. Amsterdam: Partnerschap Overgewicht Nederland; 2012.

33. Zorginstituut Nederland. Basispakket Zvw [Basic compulsory health insurance package]. Diemen, Unknown. https://www.zorginstituutnederland.nl/Verzekerde+zorg/b/basispakket-zorgverzekeringswet-zvw. Accessed on 19 Jul 2018.

34. Berendsen BA, Hendriks MR, Verhagen EA, Schaper NC, Kremers SP, Savelberg HH. Effectiveness and cost-effectiveness of 'BeweegKuur', a combined lifestyle intervention in the Netherlands: rationale, design and methods of a randomized controlled trial. BMC Public Health. 2011;11:815.

35. Berendsen BA, Kremers SP, Savelberg HH, Schaper NC, Hendriks MR. The implementation and sustainability of a combined lifestyle intervention in primary care: mixed method process evaluation. BMC Family Practice. 2015;16(1):37.

36. Van der Meer FM, Couwenbergh BTLE, Enzing JJ, Ligtenberg G, Staal PC. De gecombineerde leefstijlinterventie: kosten, opbrengsten en de praktijk [The combined lifestyle intervention: costs, outcomes and practice]. Diemen: College voor zorgverzekeringen; 2010.

37. Ministerie van Volksgezondheid Welzijn en Sport. Herziening Zorgstelsel: Brief van de Minister van Volksgezondheid, Welzijn en Sport [Revision of Dutch health care: Letter from the Minister of Health, Welfare and Sports], Kamerstuk 29689 (nr. 326) (2011).

38. Berendsen BA. Measurement and promotion of physical activity: Evaluation of activity monitors and a multidisciplinary lifestyle intervention in primary care. [PhD dissertation]. Maastricht: Maastricht University; 2016.

39. CZ. Zorginkoopbeleid 2019 Gecombineerde leefstijlinterventie [Care procurement policy 2019 Combined lifestyle intervention]. 2018.

40. Nederlandse Diabetes Federatie. NDF Zorgstandaard. Addendum Geïndiceerde preventie van diabetes type 2 [NDF Care Guidelines. Addendum on indicated prevention of type 2 diabetes]. Rotterdam: iDrukker; 2012.

41. Platform Vitale Vaten. Zorgstandaard Cardiovasculair Risicomanagement [Care Guidelines for Cardiovascular Risk Management]. Den Haag; 2013.

42. Brug J, Van Assema P, Lechner L. Gezondheidsvoorlichting en gedragsverandering: een planmatige aanpak [Health education and behavioural change: a planned approach]. Assen: Koninklijke van Gorcum; 2017.

43. Gezonde School. Gezonde school [Healthy school]. 2017. www.gezondeschool.nl. Accessed on 17 Jul 2018.

44. Van der Meer FM, Ligtenberg G, Staal PA. Preventie bij overgewicht en obesitas: de gecombineerde leefstijlinterventie [Prevention of overweight and obesity: the combined lifestyle intervention]. Diemen: College voor Zorgverzekeringen; 2009.

45. Hassan Y, Head V, Jacob D, Bachmann MO, Diu S, Ford J. Lifestyle interventions for weight loss in adults with severe obesity: a systematic review. Clinical Obesity. 2016;6(6):395-403.

46. Wilson K, Senay I, Durantini M, Sanchez F, Hennessy M, Spring B, et al. When it comes to lifestyle recommendations, more is sometimes less: a meta-analysis of theoretical assumptions underlying the effectiveness of interventions promoting multiple behavior domain change. Psychological Bulletin. 
2015;141(2):474-509.

47. Lv N, Azar KMJ, Rosas LG, Wulfovich S, Xiao L, Ma J. Behavioral lifestyle interventions for moderate and severe obesity: A systematic review. Preventive Medicine. 2017;100(Supplement C):180-193.

48. The Diabetes Prevention Program (DPP) Research Group. 10-year follow-up of diabetes incidence and weight loss in the Diabetes Prevention Program Outcomes Study. The Lancet. 2009;374(9702):16771686.

49. The Look AHEAD Research Group. Eight-year weight losses with an intensive lifestyle intervention: the look AHEAD study. Obesity (Silver Spring). 2014;22(1):5-13.

50. Duijzer G. Type 2 diabetes prevention from research to practice: the SLIMMER lifestyle intervention [PhD dissertation]. Wageningen: Wageningen University; 2016.

51. Bukman AJ. Targeting persons with low socioeconomic status of different ethnic origins with lifestyle interventions: opportunities and effectiveness [PhD dissertation]. Wageningen: Wageningen University; 2016.

52. Sacher PM, Kolotourou M, Chadwick PM, Cole TJ, Lawson MS, Lucas A, et al. Randomized controlled trial of the MEND program: a family-based community intervention for childhood obesity. Obesity (Silver Spring). 2010;18 Suppl 1:S62-68.

53. Niemer S, Bruggers C, Van den Eynde E. LEFF 2013 t/m 2015 - Een beschrijving van de ontwikkeling, uitvoering en eerste resultaten van LEFF in Nederland [LEFF 2013-2015 - A description of the development, implementation and first results of LEFF in the Netherlands]. Amsterdam: Care for Obesity \& Vrije Universiteit; 2015.

54. Gerards SM, Dagnelie PC, Gubbels JS, van Buuren S, Hamers FJ, Jansen MW, et al. The effectiveness of lifestyle triple P in the Netherlands: a randomized controlled trial. PLoS One. 2015;10(4):e0122240.

55. West F, Sanders MR, Cleghorn GJ, Davies PSW. Randomised clinical trial of a family-based lifestyle intervention for childhood obesity involving parents as the exclusive agents of change. Behaviour Research and Therapy. 2010;48(12):1170-1179.

56. Bartelink NH, Jansen MW, Kremers SP, Mulkens S, Mujakovic S. Long-term effects of the RealFit intervention on body composition, aerobic fitness, and behavior. Childhood Obesity. 2014;10(5):383-391.

57. Rijks JM, Plat J, Mensink RP, Dorenbos E, Buurman WA, Vreugdenhil AC. Children With Morbid Obesity Benefit Equally as Children With Overweight and Obesity From an Ongoing Care Program. The Journal of Clinical Endocrinology and Metabolism. 2015;100(9):3572-3580.

58. Gillies C, Abrams K, Lambert P, Cooper N, Sutton A, Hsu R, et al. Pharmacological and lifestyle interventions to prevent or delay type 2 diabetes in people with impaired glucose tolerance: systematic review and meta-analysis. BMJ. 2007;334(7588):9.

59. Barry E, Roberts S, Oke J, Vijayaraghavan S, Normansell R, Greenhalgh T. Efficacy and effectiveness of screen and treat policies in prevention of type 2 diabetes: systematic review and meta-analysis of screening tests and interventions. BMJ. 2017;356.

60. Janicke DM, Steele RG, Gayes LA, Lim CS, Clifford LM, Schneider EM, et al. Systematic review and meta-analysis of comprehensive behavioral family lifestyle interventions addressing pediatric obesity. Journal of Pediatric Psychology. 2014;39(8):809-825.

61. McGovern L, Johnson JN, Paulo R, Hettinger A, Singhal V, Kamath C, et al. Clinical review: treatment of pediatric obesity: a systematic review and meta-analysis of randomized trials. The Journal of Clinical Endocrinology and Metabolism. 2008;93(12):4600-4605.

62. Van der Heijden LB, Feskens EJM, Janse AJ. Maintenance interventions for overweight or obesity in children: a systematic review and meta-analysis. Obesity reviews: an official journal of the International Association for the Study of Obesity. 2018;19(6):798-809.

63. Harting J, van Assema P, Ruland E, van Limpt P, Gorgels T, van Ree J, et al. Implementation of an Innovative Health Service: A "Real-World" Diffusion Study. American Journal of Preventive Medicine. 2005;29(2):113-119.

64. Hawe P, Shiell A, Riley T. Theorising Interventions as Events in Systems. American Journal of Community Psychology. 2009;43(3-4):267-276.

65. Ali MK, Echouffo-Tcheugui J, Williamson DF. How effective were lifestyle interventions in real-world settings that were modeled on the Diabetes Prevention Program? Health Affairs. 2012;31(1):67-75.

66. Ackermann RT, Marrero DG. Adapting the Diabetes Prevention Program lifestyle intervention for delivery in the community: the YMCA model. The Diabetes Educator. 2007;33(1):69, 74-65, 77-68.

67. Bozack A, Millstein S, Garcel JM, Kelly K, Ruberto R, Weiss L. Implementation and Outcomes of the New York State YMCA Diabetes Prevention Program: A Multisite Community-Based Translation, 2010-2012. Preventing Chronic Disease. 2014;11:E115.

68. Duijzer G, Haveman-Nies A, Jansen SC, Ter Beek J, Van Bruggen R, Willink MGJ. SLIMMER diabetes voor- 
komen in de eerste lijn [SLIMMER preventing diabetes in the primary care]. Huisarts en Wetenschap. 2017;60(4):160-163.

69. Verstuyf J, Vansteenkiste M. Does the quality of motivation matter ? An examination of the relationship between goal content. [PhD dissertation]. Gent: Universiteit Gent; 2014.

70. Teuscher D, Bukman AJ, van Baak MA, Feskens EJM, Renes RJ, Meershoek A. A lifestyle intervention study targeting individuals with low socioeconomic status of different ethnic origins: important aspects for successful implementation. BMC Public Health. 2017;18(1):54.

71. Barte JCM, Hendriks MRC, Rutten G, Veenhof C, Bemelmans WJE. Implementation of the 'BeweegKuur' in practice: utilization of care of a lifestyle intervention in the Netherlands. International Journal of Health Promotion and Education. 2014;52(4):222-228.

72. Helmink JH, Kremers SP, Van Boekel LC, Van Brussel-Visser FN, Preller L, De Vries NK. The BeweegKuur programme: a qualitative study of promoting and impeding factors for successful implementation of a primary health care lifestyle intervention for overweight and obese people. Family Practice. 2012;29 Suppl 1:i68-i74.

73. Lucas PJ, Curtis-Tyler K, Arai L, Stapley S, Fagg J, Roberts H. What works in practice: user and provider perspectives on the acceptability, affordability, implementation, and impact of a family-based intervention for child overweight and obesity delivered at scale. BMC Public Health. 2014;14(1):614.

74. Gerards SMPL, Kremers SPJ. The Role of Food Parenting Skills and the Home Food Environment in Children's Weight Gain and Obesity. Current Obesity Reports. 2015;4(1):30-36.

75. Storm I, Verweij A, Lucht Fvd. Integraal gezondheidsbeleid op lokaal niveau. Wat weten we en hoe nu verder? [Integrated health at the local level. What do we know and how to continue now?]. Bilthoven: Rijksinstituut voor Volksgezondheid en Milieu (RIVM); 2011.

76. Harting J, Kunst AE, Kwan A, Stronks K. A 'health broker' role as a catalyst of change to promote health: an experiment in deprived Dutch neighbourhoods. Health Promotion International. 2011;26(1):65-81.

77. Krebbekx W, Harting J, Stronks K. Does collaborative research enhance the integration of research, policy and practice? The case of the Dutch Health Broker Partnership. Journal of Health Services Research \& Policy. 2012;17(4):219-226.

78. Van Rinsum CE, Gerards SMPL, Rutten GM, Van de Goor LAM, Kremers SPJ. Coaching op Leefstijl (CooL): Eindrapportage van een implementatie- en monitoringstudie [Coaching on Lifestyle (CooL): Final report of an implementation and monitoring study]. Maastricht: Maastricht University; 2018.

79. Hill JO, Wyatt HR, Peters JC. Energy Balance and Obesity. Circulation. 2012;126(1):126-132.

80. Roumen C, Corpeleijn E, Feskens EJ, Mensink M, Saris WH, Blaak EE. Impact of 3-year lifestyle intervention on postprandial glucose metabolism: the SLIM study. Diabetic Medicine: a Journal of the British Diabetic Association. 2008;25(5):597-605.

81. Ball GD, Mackenzie-Rife KA, Newton MS, Alloway CA, Slack JM, Plotnikoff RC, et al. One-on-one lifestyle coaching for managing adolescent obesity: Findings from a pilot, randomized controlled trial in a real-world, clinical setting. Paediatrics \& Child Health. 2011;16(6):345-350.

82. The Diabetes Prevention Program Research Group. The Diabetes Prevention Program (DPP): Description of lifestyle intervention. Diabetes Care. 2002;25(12):2165-2171.

83. Rutten GM, Meis JJ, Hendriks MR, Hamers FJ, Veenhof C, Kremers SP. The contribution of lifestyle coaching of overweight patients in primary care to more autonomous motivation for physical activity and healthy dietary behaviour: results of a longitudinal study. International Journal of Behavioral Nutrition and Physical Activity. 2014;11:86.

84. Willeboordse M, Jansen MW, van den Heijkant SN, Simons A, Winkens B, de Groot RH, et al. The Healthy Primary School of the Future: study protocol of a quasi-experimental study. BMC Public Health. 2016;16:639.

85. Gerards SMPL. Childhood obesity prevention. Rationale, implementation and effectiveness of the Lifestyle Triple P intervention. [PhD dissertation]. Maastricht: Maastricht University; 2014.

86. Helmink JHM. Ready Set Go? A study of the development and implementation process of the BeweegKuur [PhD dissertation]. Maastricht: Maastricht University; 2012.

87. Benet-Martinez V, John OP. Los Cinco Grandes across cultures and ethnic groups: multitrait multimethod analyses of the Big Five in Spanish and English. Journal of Personality and Social Psychology. 1998;75(3):729-750.

88. Denissen JJ, Geenen R, van Aken MA, Gosling SD, Potter J. Development and validation of a Dutch translation of the Big Five Inventory (BFI). Journal of Personality Assessment. 2008;90(2):152-157.

89. Ryan RM, Deci EL. Self-determination theory and the facilitation of intrinsic motivation, social development, and well-being. The American Psychologist. 2000;55(1):68-78.

90. Wilson PM, Rogers WT, Rodgers WM, Wild TC. The Psychological Need Satisfaction in Exercise Scale. 
Journal of Sport \& Exercise Psychology. 2006;28(3):231-251.

91. Wilson PM, Sabiston CM, Mack DE, Blanchard CM. On the nature and function of scoring protocols used in exercise motivation research: An empirical study of the behavioral regulation in exercise questionnaire. Psychology of Sport and Exercise. 2012;13(5):614-622.

92. Pelletier L, Dion S, Slovinec-D’Angelo M, Reid R. Why Do You Regulate What You Eat? Relationships Between Forms of Regulation, Eating Behaviors, Sustained Dietary Behavior Change, and Psychological Adjustment. Motivation and Emotion. 2004;28(3):245-277.

93. Craig CL, Marshall AL, Sjostrom M, Bauman AE, Booth ML, Ainsworth BE, et al. International physical activity questionnaire: 12-country reliability and validity. Medicine and Science in Sports and Exercise. 2003;35(8):1381-1395.

94. Marshall AL, Miller YD, Burton NW, Brown WJ. Measuring total and domain-specific sitting: a study of reliability and validity. Medicine and Science in Sports and Exercise. 2010;42(6):1094-1102.

95. Van Assema P, Brug J, Ronda G, Steenhuis I. The relative validity of a short Dutch questionnaire as a means to categorize adults and adolescents to total and saturated fat intake. Journal of Human Nutrition and Dietetics. 2001;14(5):377-390.

96. The EuroQol Group. EuroQol: A new facility for the measurement of health-related quality of life. Health Policy. 1990;16(3):199-208.

97. Simonds J. The Role of Reward Sensitivity and Response Execution in Childhood Extraversion [PhD dissertation]. Eugene, OR: University of Oregon; 2006.

98. Sleddens EF, O'Connor TM, Watson KB, Hughes SO, Power TG, Thijs C, et al. Development of the Comprehensive General Parenting Questionnaire for caregivers of 5-13 year olds. International Journal of Behavioral Nutrition and Physical Activity. 2014;11:15.

99. West F, Morawska A, Joughin K. The Lifestyle Behaviour Checklist: evaluation of the factor structure. Child: Care, Health and Development. 2010;36(4):508-515.

100. Gerards SM, Hummel K, Dagnelie PC, de Vries NK, Kremers SP. Parental self-efficacy in childhood overweight: validation of the Lifestyle Behavior Checklist in the Netherlands. International Journal of Behavioral Nutrition and Physical Activity. 2013;10(1):7.

101. Birch L, Fisher J, Grimm Thomas K, Markey C, Sawyer R, Johnson S. Confirmatory factor analysis of the Child Feeding Questionnaire: a measure of parental attitudes, beliefs and practices about child feeding and obesity proneness. Appetite. 2001;36:201 - 210.

102. Gubbels JS, Kremers SP, Stafleu A, de Vries SI, Goldbohm RA, Dagnelie PC, et al. Association between parenting practices and children's dietary intake, activity behavior and development of body mass index: the KOALA Birth Cohort Study. International Journal of Behavioral Nutrition and Physical Activity. 2011;8(1):18.

103. Musher-Eizenman D, Holub S. Comprehensive Feeding Practices Questionnaire: validation of a new measure of parental feeding practices. Journal of Pediatric Psychology. 2007;32(8):960-972.

104. Gattshall ML, Shoup JA, Marshall JA, Crane LA, Estabrooks PA. Validation of a survey instrument to assess home environments for physical activity and healthy eating in overweight children. International Journal of Behavioral Nutrition and Physical Activity. 2008;5:3-3.

105. Wardle J, Sanderson S, Guthrie CA, Rapoport L, Plomin R. Parental feeding style and the inter-generational transmission of obesity risk. Obesity Research. 2002;10(6):453-462.

106. Ogden J, Reynolds R, Smith A. Expanding the concept of parental control: a role for overt and covert control in children's snacking behaviour? Appetite. 2006;47(1):100-106.

107. Lokale en Nationale Monitor Jeugdgezondheid. Standaardvraagstelling Bewegen [Standard Questionnaire on Physical Activity]. 2005. https://www.monitorgezondheid.nl/vragenlijsten/vragenlijsten-jeugd. Accessed on $15 \mathrm{Jul} 2014$.

108. Lokale en Nationale Monitor Jeugdgezondheid. Standaardvraagstelling Voeding [Standard Questionnaire on Nutrition]. 2005. https://www.monitorgezondheid.nl/vragenlijsten/vragenlijsten-jeugd. Accessed on 15 Jul 2014.

109. Lokale en Nationale Monitor Jeugdgezondheid. Standaardvraagstelling Slaapproblemen [Standard Questionnaire on Sleeping Problems]. 2006. https://www.monitorgezondheid.nl/vragenlijsten/vragenlijsten-jeugd. Accessed on 15 Jul 2014.

110. Kolotkin RL, Zeller M, Modi AC, Samsa GP, Quinlan NP, Yanovski JA, et al. Assessing weight-related quality of life in adolescents. Obesity (Silver Spring). 2006;14(3):448-457.

111. Wouters EJM, Geenen R, Kolotkin RL, Vingerhoets AJJM. Met lichaamsgewicht samenhangende kwaliteit van leven bij adolescenten [Body-weight related quality of life in adolescents]. Tijdschrift Kindergeneeskunde. 2010;78(3):119-125.

112. Schonbeck Y, Talma H, van Dommelen P, Bakker B, Buitendijk SE, Hirasing RA, et al. Increase in preva- 
lence of overweight in Dutch children and adolescents: a comparison of nationwide growth studies in 1980, 1997 and 2009. PLoS One. 2011;6.

113. Cole T, Bellizzi M, Flegal K, Dietz W. Establishing a standard definition for child overweight and obesity worldwide: international survey. BMJ. 2000;320:1240-1243.

114. Truby H, Paxton SJ. The Children's Body Image Scale: reliability and use with international standards for body mass index. The British Journal Of Clinical Psychology. 2008;47(Pt 1):119-124.

115. Moore JB, Yin Z, Hanes J, Duda J, Gutin B, Barbeau P. Measuring Enjoyment of Physical Activity in Children: Validation of the Physical Activity Enjoyment Scale. Journal of Applied Sport Psychology. 2009;21(S1):S116-S129.

116. Jurg ME, Kremers SP, Candel MJ, Van der Wal MF, De Meij JS. A controlled trial of a school-based environmental intervention to improve physical activity in Dutch children: JUMP-in, kids in motion. Health Promotion International. 2006;21(4):320-330.

117. Verplanken B, Orbell S. Reflections on Past Behavior: A Self-Report Index of Habit Strength. Journal of Applied Social Psychology. 2003;33(6):1313-1330.

118. Schaufeli WB, Bakker AB, Salanova $M$. The measurement of work engagement with a short questionnaire: A cross-national study. Educational and Psychological Measurement. 2006;66(4):701-716.

119. Beroepsvereniging Leefstijlcoaches Nederland. Het profiel van de hbo leefstijlcoach [The profile of the higher professional lifestyle coach]. 2016. http://www.blcn.nl/wp-content/uploads/2016/04/Het-profiel-van-de-hbo-leefstijlcoach-versie-1.0.pdf. Accessed on 29 Mar 2016.

120. VSNU-NOA. Competentie Instrument voor de Nederlandse Universiteiten [Competence Instrument for the Dutch Universities]. 2010. http://www.vsnu.nl/files/documenten/CAO/Competentie_Instrument_ VSNU_20101201.pdf. Accessed on 8 Sept 2015.

121. Brown T, Avenell A, Edmunds LD, Moore H, Whittaker V, Avery L, et al. Systematic review of long-term lifestyle interventions to prevent weight gain and morbidity in adults. Obesity reviews: an official journal of the International Association for the Study of Obesity. 2009;10(6):627-638.

122. Tuomilehto J, Lindström J, Eriksson JG, Valle TT, Hämäläinen H, llanne-Parikka $P$, et al. Prevention of type 2 diabetes mellitus by changes in lifestyle among subjects with impaired glucose tolerance. New England Journal of Medicine. 2001;344(18):1343-1350.

123. Van Rinsum CE, Gerards SMPL, Rutten GM, Van de Goor LAM, Kremers SPJ. The coaching on lifestyle (CooL) intervention for obesity, a study protocol for an action-oriented mixed-methods study. BMC Public Health. 2018;18(1):117.

124. Kwaliteitsinstituut voor de Gezondheidszorg CBO. Richtlijn Diagnostiek en behandeling van obesitas bij volwassenen en kinderen [Guideline Diagnostics and treatment of obesity among adults and children]. Alphen aan den Rijn; 2008.

125. ZonMw. Mooie plannen en weerbarstige praktijk. Theorie en praktijk ervaringen rond implementeren van zorgverbeteringen [Beautiful plans and stubborn practice. Theory and practical experiences of implementing care improvements]. Den Haag; 2010.

126. Sanders M, Markie-Dadds C, Turner K. Theoretical, scientific and clinical foundations of the Triple P-Positive Parenting Program: a population approach to the promotion of parenting competence. Parenting Research and Practice Monograph. 2003;1:1-24.

127. Halberstadt J. Long-term weight management in children and adolescents with severe obesity: psychological aspects [PhD dissertation]. Amsterdam: VU University Amsterdam; 2015.

128. Cullinan J, Cawley J. Parental misclassification of child overweight/obese status: The role of parental education and parental weight status. Economics and Human Biology. 2017;24:92-103.

129. Dejonghe LAL, Becker J, Froboese I, Schaller A. Long-term effectiveness of health coaching in rehabilitation and prevention: A systematic review. Patient Education and Counseling. 2017;100(9):1643-1653.

130. Hill B, Richardson B, Skouteris H. Do we know how to design effective health coaching interventions: a systematic review of the state of the literature. American Journal of Health Promotion. 2015;29(5):e158168.

131. Kivelä K, Elo S, Kyngäs $H$, Kääriäinen M. The effects of health coaching on adult patients with chronic diseases: a systematic review. Patient Education and Counseling. 2014;97(2):147-157.

132. Olsen JM, Nesbitt BJ. Health coaching to improve healthy lifestyle behaviors: an integrative review. American Journal of Health Promotion. 2010;25(1):e1-e12.

133. Samdal GB, Eide GE, Barth T, Williams G, Meland E. Effective behaviour change techniques for physical activity and healthy eating in overweight and obese adults; systematic review and meta-regression analyses. International Journal of Behavioral Nutrition and Physical Activity. 2017;14(1):42.

134. Neuner-Jehle S, Schmid M, Gruninger U. The "Health Coaching" programme: a new patient-centred and visually supported approach for health behaviour change in primary care. BMC Family Practice. 
2013;14:100

135. Lipsey M. Design Sensitivity: Statistical Power for Experimental Research. Newbury Park, CA: Sage Publications; 1990.

136. IBM Corp. IBM SPSS Statistics for Windows, Version 23.0. Armonk, NY: IBM Corp.; 2015.

137. Mensink M, Corpeleijn E, Feskens EJ, Kruijshoop M, Saris WH, de Bruin TW, et al. Study on lifestyle-intervention and impaired glucose tolerance Maastricht (SLIM): design and screening results. Diabetes Research and Clinical Practice. 2003;61(1):49-58.

138. Helmink JHM, Meis JJM, Kremers SPJ. Een jaar BeweegKuur, en dan? Een onderzoek naar de bevorderende en belemmerende factoren [A year BeweegKuur, and then? A study on the facilitators and barriers]. Universiteit Maastricht; 2010.

139. Teuscher D. A lifestyle intervention study targeting individuals with low socioeconomic status of different ethnic origins: needs of the target group and research demands [PhD dissertation]. Maastricht: Maastricht University; 2017.

140. Foster GD, Borradaile KE, Vander Veur SS, Shantz KL, Dilks RJ, Goldbacher EM, et al. The Effects of a Commercially Available Weight Loss Program among Obese Patients with Type 2 Diabetes: A Randomized Study. Postgraduate Medicine. 2009;121(5):113-118.

141. Wadden TA, Volger S, Sarwer DB, Vetter ML, Tsai AG, Berkowitz RI, et al. A Two-Year Randomized Trial of Obesity Treatment in Primary Care Practice. New England Journal of Medicine. 2011;365(21):19691979.

142. Lin JS, O'Connor EA, Evans CV, Senger CA, Rowland MG, Groom HC. Behavioral Counseling to Promote a Healthy Lifestyle for Cardiovascular Disease Prevention in Persons With Cardiovascular Risk Factors: An Updated Systematic Evidence Review for the U.S. Preventive Services Task Force. Rockville, MD: Agency for Healthcare Research and Quality (US); 2014.

143. Pelletier LG, Dion SC. An Examination of General and Specific Motivational Mechanisms for the Relations Between Body Dissatisfaction and Eating Behaviors. Journal of Social and Clinical Psychology. 2007;26(3):303-333.

144. Teixeira PJ, Carraça EV, Markland D, Silva MN, Ryan RM. Exercise, physical activity, and self-determination theory: A systematic review. International Journal of Behavioral Nutrition and Physical Activity. 2012;9(1):78

145. Santos I, Silva MN, Teixeira PJ. A self-determination theory perspective on weight loss maintenance. The European Health Psychologist. 2016;18(5):194-199.

146. Silva MN, Markland D, Vieira PN, Coutinho SR, Carraça EV, Palmeira AL, et al. Helping overweight women become more active: Need support and motivational regulations for different forms of physical activity. Psychology of Sport and Exercise. 2010;11(6):591-601.

147. Gerards SM, Dagnelie PC, Jansen MW, De Vries NK, Kremers SP. Barriers to successful recruitment of parents of overweight children for an obesity prevention intervention: a qualitative study among youth health care professionals. BMC Family Practice. 2012;13:37.

148. Bukman AJ, Teuscher D, Meershoek A, Renes RJ, van Baak MA, Feskens EJ. Effectiveness of the MetSLIM lifestyle intervention targeting individuals of low socio-economic status and different ethnic origins with elevated waist-to-height ratio. Public Health Nutrition. 2017;20(14):2617-2628.

149. Roumen C, Feskens EJ, Corpeleijn E, Mensink M, Saris WH, Blaak EE. Predictors of lifestyle intervention outcome and dropout: the SLIM study. European Journal of Clinical Nutrition. 2011;65(10):1141-1147.

150. Seidell JC, Halberstadt J. Tegenwicht: feiten en fabels over overgewicht [Counterweight - facts and fiction about overweight]. Amsterdam: Bert Bakker; 2014.

151. Davidson $\mathrm{K}$, Vidgen $\mathrm{H}$. Why do parents enrol in a childhood obesity management program?: a qualitative study with parents of overweight and obese children. BMC Public Health. 2017;17(1):159.

152. Wilson PM, Rodgers WM, Loitz CC, Scime G. "It's Who I Am ... Really!' The Importance of Integrated Regulation in Exercise Contexts1. Journal of Applied Biobehavioral Research. 2006;11(2):79-104.

153. Lamers LM, Stalmeier PFM, McDonnell J, Krabbe PFM, van Busschbach JJ. Kwaliteit van leven meten in economische evaluaties: het Nederlands EQ-5D-tarief [Measuring quality of life in economic evaluations: the Dutch EQ-5D-rate]. Nederlands Tijdschrift voor de Geneeskunde. 2005;149(28):1574-1578.

154. Bogaards L. Motivational Regulation and Enjoyment of Physical Activity in Children: Development and Validation of a Scale [Master thesis]. Maastricht: Maastricht University; 2012.

155. Damschroder LJ, Aron DC, Keith RE, Kirsh SR, Alexander JA, Lowery JC. Fostering implementation of health services research findings into practice: a consolidated framework for advancing implementation science. Implementation Science. 2009;4(1):50.

156. Fleuren M, Wiefferink K, Paulussen T. Determinants of innovation within health care organizations: literature review and Delphi study. International journal for quality in health care: journal of the Internation- 
al Society for Quality in Health Care. 2004;16.

157. De Meij JS, van der Wal MF, van Mechelen W, Chinapaw MJ. A mixed methods process evaluation of the implementation of JUMP-in, a multilevel school-based intervention aimed at physical activity promotion. Health Promotion Practice. 2013;14(5):777-790.

158. Carroll J, Winters P, Fiscella K, Williams G, Bauch J, Clark L, et al. Process Evaluation of Practice-based Diabetes Prevention Programs: What Are the Implementation Challenges? The Diabetes Educator. 2015;41(3):271-279.

159. Hamberg-van Reenen HH, Mikolajczak J, Post NAM, Barte JCM. Inventarisatie leefstijlinterventies en ondersteuningsaanbod zorgprofessionals in de diabeteszorg. Twee projecten in het kader van het $\mathrm{Na}$ tionaal Actieprogramma Diabetes [Survey of lifestyle interventions and support offered by health care professionals in diabetes care. Two projects of the Dutch National Action programme on Diabetes]. Bilthoven: Rijksinstituut voor Volksgezondheid en Milieu; 2011.

160. Preller L, Schaars D. Generieke werkzame elementen van Gecombineerde leefstijlinterventies en duurzame uitvoerbaarheid [Generic effective components of combined lifestyle interventions and sustainable feasibility]. Ede: Kenniscentrum Sport; 2016.

161. Wu T, Gao X, Chen M, van Dam RM. Long-term effectiveness of diet-plus-exercise interventions vs. diet-only interventions for weight loss: a meta-analysis. Obesity reviews: an official journal of the International Association for the Study of Obesity. 2009;10(3):313-323.

162. Van Rinsum CE, Gerards SMPL, Rutten GM, Philippens NML, Janssen EMJ, Winkens B, et al. The Coaching on Lifestyle (CooL) Intervention for Overweight and Obesity: A Longitudinal Study into Participants' Lifestyle Changes. International Journal of Environmental Research and Public Health. 2018;15(4):680.

163. Rogers EM. Diffusion of Innovations. New York: Free Press; 2003.

164. Schaufeli W, Bakker A. Voorlopige handleiding Utrechtse bevlogenheidsschaal (UBES) [Provisional Instruction Manual Utrecht's Work Engagement Scale (UWES)]. Utrecht: Universiteit Utrecht; 2003.

165. Van Dam-Nolen DHK. Voeding en leefstijl in de opleiding geneeskunde: een overzicht [Nutrition and lifestyle in medical education: an overview]. Den Haag: Ministerie van Volksgezondheid, Welzijn en Sport; 2017.

166. Leemrijse C, Veenhof C, Dekker J. Inventarisatie van onderzoeksprioriteiten voor de fysiotherapie [Survey of research priorities for physiotherapy]. Utrecht: NIVEL; 2012.

167. Ministerie voor Medische Zorg. Herziening Zorgstelsel: Brief van de Minister voor Medische Zorg [Revision of Dutch health care system: Letter from the Minister for Medical Care], Kamerstuk 29689 (nr. 909) (2018).

168. Bartholomew Eldredge LK, Markham CM, Ruiter RAC, Fernández ME, Kok G, Parcel GS. Planning Health Promotion Programs: An Intervention Mapping Approach. 3th ed. Hoboken, NJ: Wiley; 2011.

169. Lau R, Stevenson F, Ong BN, Dziedzic K, Treweek S, Eldridge S, et al. Achieving change in primary care - causes of the evidence to practice gap: systematic reviews of reviews. Implementation Science. 2016;11(1):40.

170. Van Dongen EJ, Duijzer G, Jansen SC, Ter Beek J, Huijg JM, Leerlooijer JN, et al. Process evaluation of a randomised controlled trial of a diabetes prevention intervention in Dutch primary health care: the SLIMMER study. Public Health Nutrition. 2016;19(16):3027-3038.

171. Rogers EM. Diffusion of preventive innovations. Addictive Behaviors. 2002;27(6):989-993.

172. Kim KK, Yeong L-L, Caterson ID, Harris MF. Analysis of factors influencing general practitioners' decision to refer obese patients in Australia: a qualitative study. BMC Family Practice. 2015;16(1):45.

173. Long JC, Cunningham FC, Braithwaite J. Bridges, brokers and boundary spanners in collaborative networks: a systematic review. BMC Health Services Research. 2013;13(1):158.

174. Van Rinsum CE, Gerards SMPL, Rutten GM, Van de Goor IAM, Kremers SPJ. Health Brokers: How Can They Help Deal with the Wickedness of Public Health Problems? BioMed Research International. 2017;2017:10.

175. Durlak JA, DuPre EP. Implementation Matters: A Review of Research on the Influence of Implementation on Program Outcomes and the Factors Affecting Implementation. American Journal of Community Psychology. 2008;41(3):327.

176. Buratta L, Reginato E, Ranucci C, Pippi R, Aiello C, Sbroma Tomaro E, et al. Stage of Change and Motivation to a Healthier Lifestyle before and after an Intensive Lifestyle Intervention. Journal of Obesity. 2016;2016:7.

177. Ceccarini M, Borrello M, Pietrabissa G, Manzoni GM, Castelnuovo G. Assessing motivation and readiness to change for weight management and control: an in-depth evaluation of three sets of instruments. Frontiers in Psychology. 2015;6(511).

178. Rubak S, Sandbæk A, Lauritzen T, Christensen B. Motivational interviewing: a systematic review and 
meta-analysis. The British Journal of General Practice. 2005;55(513):305-312.

179. Hendriks AM, Jansen MW, Gubbels JS, De Vries NK, Paulussen T, Kremers SP. Proposing a conceptual framework for integrated local public health policy, applied to childhood obesity - the behavior change ball. Implementation Science. 2013;8:46.

180. Rutter H, Savona N, Glonti K, Bibby J, Cummins S, Finegood DT, et al. The need for a complex systems model of evidence for public health. The Lancet. 2017;390(10112):2602-2604.

181. Moore GF, Audrey S, Barker M, Bond L, Bonell C, Hardeman W, et al. Process evaluation of complex interventions: Medical Research Council guidance. British Medical Journal. 2015;350.

182. Ng M, Fleming T, Robinson M, Thomson B, Graetz N, Margono C, et al. Global, regional, and national prevalence of overweight and obesity in children and adults during 1980-2013: a systematic analysis for the Global Burden of Disease Study 2013. The Lancet. 2014;384(9945):766-781.

183. Britz B, Siegfried W, Ziegler A, Lamertz C, Herpertz-Dahlmann BM, Remschmidt H, et al. Rates of psychiatric disorders in a clinical study group of adolescents with extreme obesity and in obese adolescents ascertained via a population based study. International journal of obesity and related metabolic disorders: journal of the International Association for the Study of Obesity. 2000;24(12):1707-1714.

184. Bray GA. Medical consequences of obesity. The Journal of Clinical Endocrinology and Metabolism. 2004;89(6):2583-2589.

185. Anderson PM, Butcher KE. Childhood obesity: trends and potential causes. Future Child. 2006;16(1):1945.

186. Kickbusch I, McCann W, Sherbon T. Adelaide revisited: from healthy public policy to Health in All Policies. Health Promotion International. 2008;23(1):1-4.

187. Ten Dam J. Gezond Beleid. Tijdschrift voor Gezondheidswetenschappen. 2010;88(5):228-231.

188. Koelen MA, Vaandrager L, Wagemakers A. The Healthy ALLiances (HALL) framework: prerequisites for success. Family Practice. 2012;29 Suppl 1:i132-i138.

189. Hendriks AM, Gubbels JS, De Vries NK, Seidell JC, Kremers SPJ, Jansen MWJ. Interventions to Promote an Integrated Approach to Public Health Problems: An Application to Childhood Obesity. Journal of Environmental and Public Health. 2012;2012:913236.

190. Weber EP, Khademian AM. Wicked Problems, Knowledge Challenges, and Collaborative Capacity Builders in Network Settings. Public Administration Review. 2008;68(2):334-349.

191. Huang TT, Drewnowski A, Kumanyika SK, Glass TA. A Systems-Oriented Multilevel Framework for Addressing Obesity in the 21st Century. Preventing Chronic Disease. 2009;6(3):A82.

192. Glass TA, McAtee MJ. Behavioral science at the crossroads in public health: extending horizons, envisioning the future. Social Science \& Medicine. 2006;62(7):1650-1671.

193. Kickbusch I, Buckett K. Implementing Health in All Policies: Adelaide 2010. Adelaide: Government of South Australia; 2010.

194. Kumanyika SK, Morssink CB. Bridging Domains in Efforts to Reduce Disparities in Health and Health Care. Health Education \& Behavior. 2006;33(4):440-458.

195. Borys JM, Le Bodo Y, Jebb SA, Seidell JC, Summerbell C, Richard D, et al. EPODE approach for childhood obesity prevention: methods, progress and international development. Obesity Reviews. 2012;13(4):299-315.

196. Williams P. Special Agents: The Nature and Role of Boundary Spanners. Paper to the ESRC Research Seminar Series - Collaborative Futures: New Insights from Intra and Inter-Sectoral Collaborations. 2010.

197. Steenbakkers M. Lokaal integraal gezondheidsbeleid: realistische uitdaging of utopie? Een onderzoek binnen gemeenten naar mogelijkheden tot intersectorale samenwerking. [Integrating Health in all Policies: realistic challenge or utopia? A study of the opportunities for inter-sectoral collaboration within Dutch municipal governments to stimulate integrated health policy] [PhD dissertation]. Maastricht: Maastricht University; 2012.

198. De Leeuw E. Healthy Cities: urban social entrepreneurship for health. Health Promotion International. 1999;14(3):261-270.

199. Linger RJ, Bakker I, Bosscher RJ, ten Dam JJM. De invloed van de fysieke omgeving op lichamelijke activiteit: hoe beweeg je beleidsmakers? [The influence of the physical environment on physical activity: how do you get policy makers moving?]. Tijdschrift voor Gezondheidswetenschappen. 2011;89(6):300-304.

200. Michie S, van Stralen M, West R. The behaviour change wheel: A new method for characterising and designing behaviour change interventions. Implementation Science. 2011;6(1):42.

201. Ter Denge PJ. Gezondheidsmakelaar als vliegwiel. Een evaluatieonderzoek naar het project Gezondheidsmakelaars in Nijmegen. [Health brokers as a flywheel. An evaluation study of the health brokers project in Nijmegen]. Nijmegen: Municipality Nijmegen and MHS Nijmegen; 2012.

202. Himmelman AT. Collaboration Defined: A Developmental Continuum of Change Strategies. 2002. http:// 
depts.washington.edu/ccph/pdf_files/4achange.pdf. Accessed on 5 Apr 2017.

203. Goodman LA. Snowball Sampling. Annals of Mathematical Statistics. 1961;32(1):148-170.

204. Creswell JW, Miller DL. Determining Validity in Qualitative Inquiry. Theory Into Practice. 2000;39(3):124130.

205. Rijksoverheid. Decentralisatie van overheidstaken naar gemeenten [Decentralisation government tasks to municipalities]. 2015. https://www.rijksoverheid.nl/onderwerpen/gemeenten/decentralisatie-van-overheidstaken-naar-gemeenten. Accessed on 29 Jan 2016.

206. Hendriks AM, Kremers SP, Gubbels JS, Raat H, de Vries NK, Jansen MW. Towards health in all policies for childhood obesity prevention. Journal of Obesity. 2013;2013:632540.

207. Alter C, Hage J. Organizations working together: coordination in interorganizational networks. Newbury Park: Sage; 1993.

208. Steenbakkers M, Jansen M, Maarse H, de Vries N. Lokaal integraal gezondheidsbeleid: effecten van beleidsondersteuning op de ontwikkeling van gemeentelijke intersectorale samenwerking. [Local integrated health policy: effects of policy support for the development of municipal inter-sectoral collaboration]. Tijdschrift voor Gezondheidswetenschappen. 2012;90(3):184-192.

209. McGuire M. Collaborative Public Management: Assessing What We Know and How We Know It. Public Administration Review. 2006;66:33-43.

210. Guldbrandsson K, Fossum B. An exploration of the theoretical concepts policy windows and policy entrepreneurs at the Swedish public health arena. Health Promotion International. 2009;24(4):434-444.

211. Roussos ST, Fawcett SB. A review of collaborative partnerships as a strategy for improving community health. Annual Review of Public Health. 2000;21:369-402.

212. Hendriks AM. Wicked problems and challenging opportunities: development of integrated public health policies for the prevention of obesity [PhD dissertation]. Maastricht: Maastricht University; 2016.

213. Burt R. Structural Holes and Good Ideas. American Journal of Sociology. 2004;110(2):349-399.

214. Agranoff R, McGuire M. Big Questions in Public Network Management Research. Journal of Public Administration Research and Theory. 2001;11(3):295-326.

215. Leenaars KE, Smit E, Wagemakers A, Molleman GR, Koelen MA. Facilitators and barriers in the collaboration between the primary care and the sport sector in order to promote physical activity: A systematic literature review. Preventive Medicine. 2015;81:460-478.

216. Leenaars KE, Smit E, Wagemakers A, Molleman GR, Koelen MA. The role of the care sport connector in the Netherlands. Health Promotion International. 2016.

217. WHO Europe. European Charter on counteracting obesity. WHO European Ministerial Conference on Counteracting Obesity; Istanbul2006.

218. Kloek CJJ, Tol J, Veenhof C, Van der Wulp I, Swinkels ICS. Huisartsenzorg bij overgewicht en obesitas [General practice care for overweight and obesity]. Huisarts en Wetenschap. 2015;58(2):66-69.

219. Cunningham FC, Ranmuthugala G, Plumb J, Georgiou A, Westbrook JI, Braithwaite J. Health professional networks as a vector for improving healthcare quality and safety: a systematic review. BMJ Quality \& Safety. 2012;21(3):239-249.

220. Greenhalgh T, Robert G, Macfarlane F, Bate P, Kyriakidou O. Diffusion of innovations in service organizations: systematic review and recommendations. The Milbank Quarterly. 2004;82(4):581-629.

221. Leenaars KEF, Smit E, Wagemakers A, Molleman GRM, Koelen MA. Exploring the impact of the care sport connector in the Netherlands. BMC Public Health. 2017;17(1):813

222. Hawe P, Shiell A, Riley T. Complex interventions: how "out of control" can a randomised controlled trial be? BMJ. 2004;328(7455):1561-1563.

223. Dera-de Bie EGE, Brink- Melis W, Jansen M, Gerver WJ. Characteristics of child health care practitioners in overweight prevention of children. Applied Nursing Research. 2016;29:157-162.

224. Van de Glind I, Bunn C, Gray CM, Hunt K, Andersen E, Jelsma J, et al. The intervention process in the European Fans in Training (EuroFIT) trial: a mixed method protocol for evaluation. Trials. 2017;18(1):356.

225. Van der Ploeg HP, Van Nassau F, Andersen E, Bunn C, Gray CM, Hunt K, et al., editors. Effectiveness of the EuroFIT lifestyle program delivered to male football fans across Europe. International Society of Behavioral Nutrition and Physical Activity; 2018; Hong Kong.

226. Eriksson M. Social capital and health - implications for health promotion. Global Health Action. 2011;4(1):5611.

227. Huffman M. Health coaching: a new and exciting technique to enhance patient self-management and improve outcomes. Home Healthc Nurse. 2007;25(4):271-274; quiz 275-276.

228. Maastricht UMC+. $\mathrm{COACH}$ wint Europese prijs voor beste 'Healthcare Design' [COACH wins European award for best 'HealthCare Design']. 2018. https://www.mumc.nl/actueel/nieuws/coach-wint-europese-prijs-voor-beste-healthcare-design. Accessed on 18 Jun 2018. 
229. Latta JM, Van der Meer FM. De gecombineerde leefstijlinterventie nader bekeken. Addendum bij de duiding van het Zorginstituut van 2009 [A closer look at combined lifestyle interventions. Addendum to the 2009 report of the National Health Care Institute]. Diemen: Zorginstituut Nederland; 2018.

230. BeweegKuur. Subsidieronde: monitoringonderzoek naar pakket 1 en 2 BeweegKuur: eindverslagformulier [Subsidy round: monitoring study of packages 1 and 2 of the BeweegKuur: final reporting form]. Ede: ZonMw; 2014.

231. Freund AM, Hennecke M. Changing eating behaviour vs. losing weight: The role of goal focus for weight loss in overweight women. Psychology \& Health. 2012;27(sup2):25-42.

232. Kolotourou M, Radley D, Chadwick P, Smith L, Orfanos S, Kapetanakis V, et al. Is BMI Alone a Sufficient Outcome To Evaluate Interventions for Child Obesity? Childhood Obesity. 2013;9(4):350-356.

233. Small SA, Uttal L. Action-Oriented Research: Strategies for Engaged Scholarship. Journal of Marriage and Family. 2005;67(4):936-948.

234. Schaap R, Bessems K, Otten R, Kremers S, van Nassau F. Measuring implementation fidelity of schoolbased obesity prevention programmes: a systematic review. International Journal of Behavioral Nutrition and Physical Activity. 2018;15(1):75.

235. Centraal Bureau voor de Statistiek. Bevolking; generatie, geslacht, leeftijd en migratieachtergrond, 1 januari [Population; generation, gender, age and migration background, 1 January]. Den Haag/Heerlen, 2018. http://statline.cbs.nl/StatWeb/publication/?VW=T\&DM=SLNL\&PA=37325\&D1=0\&D2=a\&D3=0\& D4=0\&D5=0-4\&D6=I\&HD=110629-1412\&HDR=G5,T,G3,G2,G4\&STB=G1. Accessed on $11 \mathrm{Jul} 2018$.

236. Philippens NML, Janssen EMJ. CooL Werkblad beschrijving interventie [CooL Workbook for description of the intervention]. Amersfoort: Expertisecentrum Leefstijlinterventies; 2018.

237. Care for Obesity. Concept landelijk model: ketenaanpak voor kinderen met overgewicht en obesitas [Draft national model: health care chain for children with overweight and obesity]. Amsterdam: Stichting Vrije Universiteit Amsterdam; 2018.

238. De Laat S. Evaluation of the preventive care chain for overweight in children. Amsterdam: Nederlands Trial Register; 2017. http://www.trialregister.nl/trialreg/admin/rctview.asp?TC=6813. Accessed on 27 Jul 2018.

239. World Health Organisation. Classification of Digital Health Interventions. Geneva: WHO; 2018.

240. Singh AS, Mulder C, Twisk JW, van Mechelen W, Chinapaw MJ. Tracking of childhood overweight into adulthood: a systematic review of the literature. Obesity reviews: an official journal of the International Association for the Study of Obesity. 2008;9(5):474-488.

241. Ter Haar WMA. Communiceren en improviseren: Omgaan met dynamiek en complexiteit bij de ontwikkeling en implementatie van een gezondheidsinterventie [Communicating and improvising: Dealing with dynamics and complexity in the development and implementation of a health intervention] [PhD dissertation]. Amsterdam: University of Amsterdam; 2014.

242. Van Giessen A, De Wit GA, Wendel-Vos W, Feenstra TL. Budget impact analyse van gecombineerde leefstijlinterventie (GLI). Raming van het benodigde budget bij opname van de GLI in de basisverzekering [Budget impact analysis of combined lifestyle intervention (CLI). Estimation of the required budget when the $\mathrm{CLI}$ is included in the basic health insurance package]. Bilthoven: Rijksinstituut voor Volksgezondheid en Milieu (RIVM); 2018.

243. Broeders D, Das D, Jennissen R, Tiemeijer W, De Visser M. WRR-Policy Brief 7. Van verschil naar potentieel. Een realistisch perspectief op de sociaaleconomische gezondheidsverschillen [WRR-Policy Letter 7. From difference to potential. A realistic perspective on socio-economic health differences]. Den Haag: Wetenschappelijke Raad voor het Regeringsbeleid (WRR); 2018.

244. Ecsplore. HealthyLIFE. 2017. https://healthylife.ecsplore.nl/. Accessed on 24 Aug 2018.

245. Eerstelijns Zorggroep Haaglanden (ELZHA). Healthy Heart. 2018. https://www.elzha.nl/zorgprogamma/ healthy-heart/. Accessed on 24 Aug 2018.

246. Keer Diabetes2 Om. Keer Diabetes2 Om. 2018. https://keerdiabetesom.nl/. Accessed on 24 Aug 2018. 



\section{DANKWOORD}

Wat ontzettend cool dat na 4,5 jaar mijn proefschrift af is. Er is veel gebeurd in die tijd en we hebben veel werk verzet. Mijn promotietraject heeft veel fases gekend. In het begin was ik vooral praktisch bezig, zoals met het opstarten van de interventie en het bijwonen van de projectgroep overleggen. Gaandeweg kwam er meer ruimte om aan het wetenschappelijk gedeelte te werken en kon ik me meer gaan focussen op het schrijven van de artikelen. Net als bij alle promovendi wisselde mijn motivatie door de jaren heen. Vooral omdat wetenschap niet met de dag komt en het lang kan duren voordat je een succes boekt. Daarvoor heb je mensen nodig die je motiveren en het gevoel geven dat je op de goede weg zit. Gelukkig had ik genoeg mensen om me heen, die me in alle fases hebben geholpen en gesteund. Ik wil iedereen bedanken die bijgedragen heeft aan mijn proefschrift!

Ik heb veel geluk gehad met mijn promotieteam. Stuk voor stuk heb ik veel aan jullie vier gehad. Mijn promotietraject begon al op een goede manier, want tijdens het sollicitatiegesprek hebben we gezellig gekletst over atletiek en hardlopen. De projectgroep overleggen in het begin voelden ook als een uitje, wanneer ik er samen met Geert of Sanne naar toe ging. Tijdens mijn promotietraject kreeg ik zowel op werk- als privévlak steeds meer vrijheid. Gelukkig kreeg ik goede sturing als ik even niet meer wist hoe ik het moest aanpakken. De teamindeling veranderde ook af en toe, wat een positief effect had op mij.

Stef, ik kon bij jou goed de ontwikkelingen in de praktijk bespreken. Je begreep goed wat er gaande was en je was ook nauw betrokken bij de discussies. Ook vond ik het erg bijzonder om te zien hoe intensief jij hebt meegeholpen en meegeschreven, zo offerde je zelfs een nationale feestdag op om samen met mij aan het eindrapport te werken.

Sanne, je was nog maar net klaar met jouw eigen promotietraject toen jij mij ging begeleiden. Maar je hebt me altijd erg goed geholpen en ik kon altijd bij jou binnenlopen voor vragen. Ik heb veel gehad aan jouw kritische en gedetailleerde manier van feedback. Ook heb ik op onderwijsgebied veel van je geleerd door bij jou mee te kijken, en toen je mij kwam observeren. Daarnaast is het echt leuk om nu het moederschap samen te kunnen delen.

Geert, je bent altijd in voor een Brabants grapje. Het is fijn dat je ondanks de afstand toch zo betrokken bent gebleven, toen je uit Maastricht wegging. Het is altijd leuk om je weer te zien bij teamoverleggen. Ook inhoudelijk was jouw ondersteuning fijn, door jouw nuttige en gedetailleerde feedback.

len, je stond wat meer op de achtergrond, maar toch bleef je altijd goed op de hoogte van alle ontwikkelingen. Vooral rondom de procesevaluatie hebben we veel gediscussieerd en gaf je me altijd goede inzichten. Je kan dingen goed en gestructureerd opschrijven en je kijkt naar de rode lijn in het verhaal. Doordat jij in mijn team zat heb ik ook een tijdje regelmatig in Tilburg gewerkt. Bij Tranzo voelde ik me altijd erg thuis en vond ik de sfeer erg gemoedelijk. 
Zonder mijn onderzoeksassistenten had ik dit allemaal niet voor elkaar gekregen. Lieke, Else, Sabine, Floor en Anke, bedankt voor al jullie werk en inspanningen. Zo hebben jullie deelnemers ingepland en opgemeten, ze nagebeld om ze aan de vragenlijsten te herinneren, vragenlijsten ingevoerd, interviews uitgetypt, interviews gecodeerd en nog zoveel meer. Vooral Lieke, je hebt onwijs veel bijgedragen aan het project en het was fijn om jou als discussiemaatje te hebben. Wesley, jij had me in een korte tijd erg goed geholpen met de gegevens uit de huisartssystemen.

Nina, je was het beste kamergenootje wat ik me kon wensen. We hebben ontzettend veel met elkaar gedeeld. Ik heb veel aan jou gehad dankzij onze soortgelijke onderzoeken en het feit dat we ongeveer op hetzelfde moment met hetzelfde onderwerp bezig waren. We konden elkaar goed advies geven en je was mijn sparringpartner voor alles. Ik zal ook ons nachtje samen in Wageningen tijdens een cursus niet vergeten, net als de treinreizen samen.

Ook mijn andere kamergenootjes, Robin en Dorus, bedankt. Robin, je wist overal antwoord op toen ik net nieuw was op de afdeling. We hebben samen een leuke tijd gehad en we hebben vaak samen gesport. Dorus, leuk dat je weer terugkwam op onze afdeling, erg gezellig om bij jou op de kamer te zitten. Ook is het heel leuk om alles rondom onze baby's te bespreken.

Ik wil ook andere (oud)collega's bedanken, een aantal wil ik nog even specifiek benoemen. Dennis, ik heb jou niet voor niks gevraagd als paranimf. Je bent de gangmaker van onze afdeling en altijd erg geïnteresseerd. Je kon me ook vaak helpen met statistiek of EndNote en je dacht ook graag mee met mijn teksten. We hebben ook veel leuke dingen samengedaan, zoals rennen, boulderen en eten, wat we ook vaak met Nina erbij deden. Anna Marie, je hebt mij niet alleen geholpen met mijn master thesis, je was ook nauw betrokken bij het artikel over de gezondheidsmakelaars. Daarnaast hebben we vaak samen gesport en gezellig gegeten. Liesbeth, het artikel ligt er nog niet, maar je hebt wel veel geholpen bij hoe ik goed het netwerk in de regio's kon onderzoeken. En alle collega's bedankt die feedback hebben gegeven op mijn stukken tijdens de PhD lunch.

Alle overige coauteurs, Nicole, Ester, Bjorn en Madelon, bedankt voor jullie bijdrage bij het meelezen en meeschrijven van de artikelen. Nicole en Ester, jullie nog extra bedankt voor jullie inzet bij de landelijke discussies en het indienen van CooL bij het Loket Gezond Leven. Bjorn, jij stond altijd klaar om de statistische vraagstukken op te lossen en begeleidde mij goed in het analyseren van de data.

Madelon en Geert, dankzij jullie innoverende denkwijze is het CooL project opgestart en zullen gecombineerde leefstijlinterventies vergoed gaan worden. Madelon, je bedacht kritische onderzoeksvragen en dacht vervolgens fijn mee over hoe we achter het antwoord konden komen. Ook zocht je altijd naar nieuwe manieren hoe stagiaires (Sarina en Ide) ons verder konden helpen.

Koen, jij hebt je als projectleider altijd erg ingezet voor het onderzoek, waarbij je opzoek ging naar praktische oplossingen. Ook toen jouw rol wat minder werd in de pilot, stond 
jij altijd klaar om antwoorden te geven op mijn vragen. Het was erg fijn om met jou zo intensief samen te werken.

Wat heb ik een fijn contact met alle leefstijlcoaches in onze pilot gehad en wat leuk om bij jullie mee te mogen kijken en meer te leren over leefstijlcoaching. Jullie hebben mijn werk zeker leuker gemaakt. Soms voelde ik me wel schuldig als ik weer wat van jullie vroeg. Het was voor jullie veel extra werk om met het onderzoek mee te doen, al die administratie, interviews en vragenlijsten die jullie zelf moesten invullen. Jullie hebben ook hard gewerkt om alle vragenlijsten van de deelnemers goed ingevuld terug te krijgen.

Bedankt voor jullie bijdrage en inzet ook aan alle andere leden uit de projectgroepen en de stuurgroep, waaronder de medewerkers van zorggroepen, GGD'en, CZ, gemeenten, beweegpartners en artsen, een diëtiste en een verpleegkundige. Zonder jullie hulp was het niet gelukt om CooL in de verschillende regio's te implementeren.

Jenneke en allebei de Sannes, we hebben niet vaak met $z^{\prime} n$ vieren als promotiegroepje bij elkaar gezeten. Toch vond ik het erg gezellig en handig om van elkaar te weten waar jullie mee bezig waren, zo konden we elkaar een beetje verder helpen en sparren over onze gemeenschappelijke onderwerpen. Veel succes nog met jullie promotietrajecten.

Toen ik wat vaker bij Tranzo kwam, kon ik altijd gezellig een praatje maken met Andrea en Hilde. Ik zat een tijdje één keer in de week tegenover jullie kantoor en jullie hebben mijn tijd bij Tranzo nog leuker gemaakt.

Jan, u corrigeerde niet alleen de diverse hoofdstukken op Engelse grammatica, u las de tekst ook met een kritische blik door. Dit heeft mij veel geholpen bij het schrijven van de teksten. Daarnaast dacht u graag mee over het juist woordgebruik en hielp u met het vertalen van het Nederlandse manuscript.

Ik heb tijdens mijn promotietraject gemerkt dat het zinvol is om van anderen te leren en ik heb veel gehad aan vrienden die mij voor gingen in het promoveren. Eveline, Martin, Ruben, Evelien, Lieke, Louise, Andrea en Kristin, ik wil jullie bedanken voor de goede adviezen, de verhalen over hoe het bij jullie is gegaan, de etentjes samen en de voorbeelden van lay-out voor het proefschrift. Kirsten, Janine, Lisa, Feike, Wouter en Famke, jullie zijn nog bezig met jullie promotietraject, maar ook jullie hebben een steentje bijgedragen door de discussies die we hebben gehad over onze onderzoeken. Ook jullie nog veel succes met jullie promotietrajecten.

Nina en Daan, ik ben jullie heel veel dankbaar. Sinds wij naar Malden verhuisd zijn, mag ik bij jullie in Maastricht blijven slapen. Ik voel me er bijna net zo thuis als in ons eigen huis. Nina, jou wil ik nog speciaal bedanken voor het meedenken met ons rapport en de andere keren dat we samen over mijn proefschrift aan het discussiëren waren.

Niet iedereen begreep wat 'promoveren' betekent, maar elke keer vroegen mijn (schoon-)familieleden er toch naar. Volgens jullie wist ik overal wel antwoord op en had 
ik verstand van medicijnen, voedingsleer en ziekten. Hopelijk hebben jullie nu een beter idee van wat ik heb gedaan in de afgelopen jaren. Uiteraard zal ik alle andere vragen ook proberen te beantwoorden, dan verzin ik wel weer een leuk antwoord.

Jan en Sigrid, wat een toeval dat jullie in één van onze pilotregio's wonen. Hierdoor heb ik vaak bij jullie kunnen werken en overnachten als ik weer een overleg in de buurt had. Ook waren jullie goede ambassadeurs van CooL, want jullie vertelden vrienden en collega's over het project.

Ceciel, ik vind het geweldig dat mijn grote zus mij met de lay-out van het proefschrift en met de figuren heeft geholpen. Het betekent veel voor me dat we dit samen hebben gedaan. Je kwam met goede ideeën over hoe we het konden vormgeven en je hebt er veel werk en tijd ingestopt. Zelf ben ik erg trots op het eindresultaat.

Mam, je was altijd erg geïnteresseerd in mijn onderzoek en stuurde daarom vaak artikelen naar mij door over gerelateerde onderzoeken en actualiteiten. Je prikkelde mij met kritische inzichten en je was altijd erg betrokken in hoe het met mijn promotie ging. Bedankt voor al jouw liefde en steun.

Pap, helaas kan je mijn promotie niet meer meemaken, maar ik weet zeker dat je heel trots op mij geweest zou zijn. Je vertelde tegen iedereen dat ik aan het promoveren was en dat ze allemaal maar naar de leefstijlcoach moesten gaan. Je had mij ook graag geholpen met het controleren van de teksten. Je blijft voor altijd in mijn gedachten bij me.

Kleine man in mijn buik, je zorgde voor een goede deadline. Door jou heb ik een eindsprintje ingezet om alles op tijd afgerond te krijgen voordat jij op de wereld komt.

Als laatste wil ik de belangrijkste persoon bedanken. Allerliefste Stefan, elke keer stond je weer voor me klaar en je was altijd een luisterend oor. Je hebt mij onwijs veel gesteund in moeilijk tijden. We zijn ook heel wat verhuisd in de afgelopen jaren, maar nu zijn we gelukkig lekker gesetteld. Uiteindelijk heb je toch stiekem best veel aan mijn proefschrift bijgedragen, voornamelijk door al mijn vragen te beantwoorden en mee te denken over hoe ik sommige dingen het beste kon aanpakken.

Ik ben heel trots op het resultaat van mijn proefschrift, maar had dit niet zonder jullie gekund. Dank jullie wel! 


\section{CURRICULUM VITAE}

Celeste Elvira van Rinsum was born in Nijmegen, the Netherlands, on December $21^{\text {st }} 1989$. In 2011 she graduated from the Bachelor of Health Sciences at Maastricht University, with a major in Health Education and Promotion and a minor in Movement Sciences. She did her electives, she at the University of Gothenburg, Sweden, Department of Social Work. During a sabbatical from her studies, she worked at the university and as a volunteer for an AIDS NGO in Kenya. She finished the Master Health Education and Promotion in 2013, as well at Maastricht University. For her master thesis she studied the role of health brokers in the Netherlands on behalf of the Northern Limburg public health services. Hereafter, she started to work at 'The Maastricht Study', which is a large study in Maastricht on type 2 diabetes and other chronical diseases, as a research assistant.

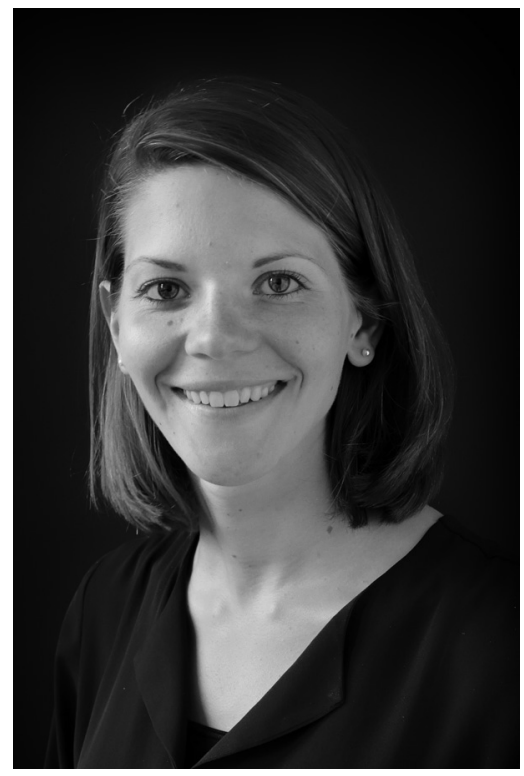

She stayed in Maastricht and in 2014 her PhD started at the department of Health Promotion of the Maastricht University, in collaboration with Tranzo (Tilburg University). During this 4.5 years PhD trajectory, she mainly investigated the CooL intervention, for which she had close contact with the lifestyle coaches and the project teams in the different regions. For one year, she was appointed as 'project leader' to steer the project groups during the execution phase. 



\section{PUBLICATION LIST}

\section{List of publications presented in this thesis}

Van Rinsum CE, Gerards SMPL, Rutten GM, Van de Goor LAM, Kremers SPJ. The coaching on lifestyle (CooL) intervention for obesity, a study protocol for an action-oriented mixed-methods study. BMC Public Health. 2018;18:117.

Van Rinsum C, Gerards S, Rutten G, Van de Goor I, Kremers S. Coaching op Leefstijl (CooL) interventie: de leefstijlcoach als spin in het web? [Coaching on Lifestyle (CooL) intervention: the lifestyle coach as a linchpin?]. Tijdschrift voor Gezondheidswetenschappen. 2018;96(5):189-193.

Van Rinsum CE, Gerards SMPL, Rutten GM, Philippens NML, Janssen EMJ, Winkens B, Van de Goor LAM, Kremers SPJ. The Coaching on Lifestyle (CooL) Intervention for Overweight and Obesity: A Longitudinal Study into Participants' Lifestyle Changes. International Journal of Environmental Research and Public Health. 2018;15(4):680.

Van Rinsum CE, Gerards SMPL, Rutten GM, Johannesma MCG, Van de Goor LAM, Kremers SPJ. The implementation of the Coaching on Lifestyle ( $\mathrm{CooL}$ ) intervention: Lessons learnt. Submitted.

Van Rinsum CE, Gerards SMPL, Rutten GM, Van de Goor IAM, Kremers SPJ. Health Brokers: How Can They Help Deal with the Wickedness of Public Health Problems? BioMed Research International. 2017;2017:1-10.

\section{Report}

Van Rinsum CE, Gerards SMPL, Rutten GM, Van de Goor LAM, Kremers SPJ. Coaching op Leefstijl (CooL): Eindrapportage van een implementatie- en monitoringstudie [Coaching on Lifestyle (CooL): Final report of an implementation and monitoring study]. Maastricht: Maastricht University; 2018. 



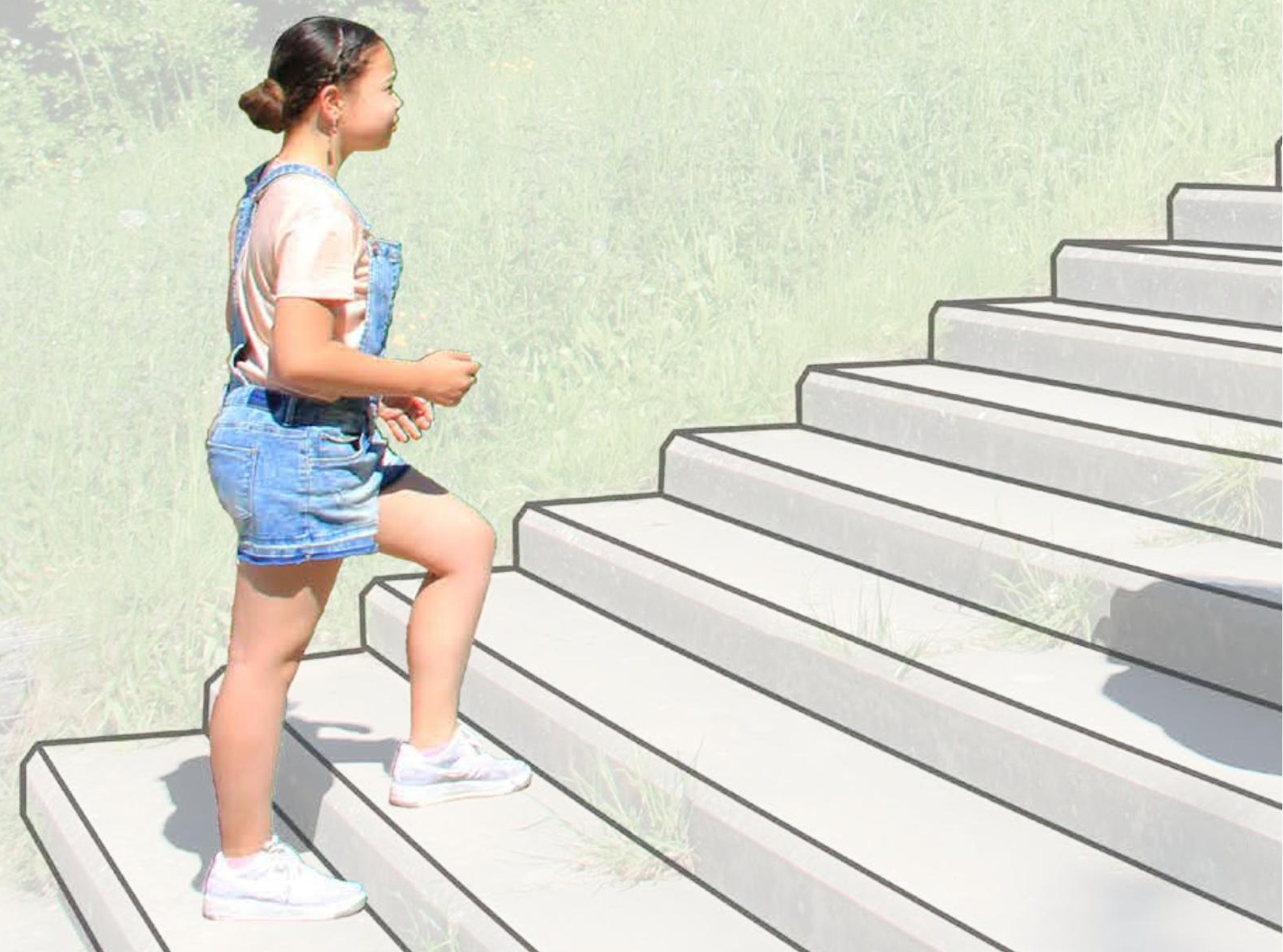

